


\title{
ICPP2018 Abstracts of Concurrent Session Presentations
}

\begin{abstract}
The abstracts are published as a supplement to Phytopathology for citation purposes. They were not reviewed by the Phytopathology Editorial Board and were not edited by the APS editorial staff. Please send questions or comments to aps@scisoc.org.
\end{abstract}

https://doi.org/10.1094/PHYTO-108-10-S1.240

(C) 2018 The American Phytopathological Society

Integrated pest management (IPM) still not sufficiently used in practice Z. Sawinska (1), J. Strzelińska (1), S. SWITEK (2), R. Głowicka-Wołoszyn (3), (1) Departament of Agronomy, Poznan University of Life Sciences, Poznan, POLAND; (2) Institute of Zoology, Poznan University of Life Sciences, Poznan, POLAND; (3) Department of Finance and Accounting, Poznan University of Life Sciences, Poznan, POLAND

Integrated Pest Management (IPM) is a strategy which encourages the reduction of pesticide use by employing a variety of pest control options to contain or manage pests below their economic injury levels. Implementation and adoption of an IPM strategy can help to reduce environmental and human health risks as well as pest management costs. Farmers' participation and engagement are crucial to the success of IPM. The research was conducted in 280 polish farms located in Great Poland region in the years 2013-2014 with the use of survey analysis. Well-developed food and dairy industry as well seed enterprises are the hallmarks of this part of Poland. Farmers were able to best recognize the symptoms of Blumeria graminis in $76,7 \%$; Puccinia recondita in 74,6\%. They worst recognize symptoms of fusarium ear blight (Fusarium spp.) in 22\% and snow mold (Microdochium nivale) in $20 \%$. As the survey farms size were growing, farmers' knowledge about pest also grew. This relationship also concerned the using IPM practice. The awareness of farmers on fungal diseases and the use of non-chemical methods of plant protection was significantly higher in the largest farms than in smaller farms. It is necessary to lead informative training campaigns on the concept of on-farm applications of IPM.

Exploiting our knowledge of how fungal plant pathogens use visible and UV light A. STENSVAND, Norwegian Institute of Bioeconomy Research (NIBIO), Ås, NORWAY

Fungal plant pathogens evolved amidst natural cycles of light and darkness. The imposition of light-filtering or blocking material such as plastics or glass, or provision of supplemental electrical lighting is a recent event in their evolutionary history. Light filtering or light supplementation can fundamentally alter pathogen development and resultant disease severity as fungi sense, interpret and respond to light. Direct damage to pathogens by natural incoming UV can be blocked by plastics and glass. Conversely, it can be strategically employed through supplementation to suppress disease. Several examples will be provided of the use of UVB and UVC in glasshouse, plastic tunnel, and open field plantings of diverse crops for suppression of powdery mildews. Experiments using UV-transparent fluorocarbon plastics indicate a pronounced and previously underestimated effect of UV exclusion on severity of powdery mildews when sunlight is filtered by glass, polyethylene, or polycarbonate films; none of which transmit UV. Visible light, particularly supplemental red and blue light, can also be used to disrupt and misdirect fungal development through disruption of circadian systems (e.g., continuous light and suppression of rose powdery mildew) and photoreceptor control of morphogenesis (e.g., red light suppression of powdery and downy mildews). We can use light to suppress disease, and refine production systems to avoid unintentionally exacerbating disease severity.

Within-plant distribution of PVY strain mixture differs spatio-temporally in potato cultivars S. MONDAL (1), S. Gray (2), (1) Cornell University, Ithaca, NY, USA; (2) Cornell University/USDA-ARS, Ithaca, NY, USA

Our recent studies indicated that the tuber necrotic strain of Potato virus $Y\left(\mathrm{PVY}^{\mathrm{NTN}}\right)$ can co-localize with the ordinary strain $\left(\mathrm{PVY}^{\mathrm{O}}\right)$ in single epidermal leaf cells of potato and tobacco plants and their cellular distribution changes spatio-temporally. To understand infection dynamics in whole plants and daughter tubers, Red Maria, Cal White, and Pike potato plants were mechanically inoculated either at pre- or at post-flowering stage with all possible heterologous combinations of two PVY ${ }^{\mathrm{O}}$ and two $\mathrm{PVY}^{\mathrm{NTN}}$ isolates. Systemically infected leaves were collected from different positions on the plant at different times after inoculation and virus composition was quantified by TAS-ELISA. Tubers were collected and tested to determine which viruses were vertically transmitted. PVY ${ }^{\mathrm{NTN}}$ outcompeted $\mathrm{PVY}^{\mathrm{O}}$ in Pike at nearly all time points and positions. Both strains were equally competitive in Red Maria and Cal White plants early in the infection when inoculated pre-flower, but PVY ${ }^{\mathrm{NTN}}$ dominated at later stages and in plants inoculated post-flower. Tuber yield was greater in plants inoculated post-flower, but regardless of inoculation time, both virus strains were present in the daughter plants. Relative titer of PVY ${ }^{\mathrm{NTN}}$ and $\mathrm{PVY}^{\mathrm{O}}$ at the later stages of mother plant development was indicative of what was found in the daughter plants. The overall dynamics of mixed infections differs among cultivars and virus isolates, but it does not change the vertical transmission outcome. 
Can nanoparticles enhance disease resistance through mineral nutrition

N. ZUVERZA-MENA (1), W. H. Elmer (2), R. De La Torre-Roche (2), L. Pagano (3), S. Majumdar (4), C. Dimkpa (5), J. Gardea-Torresdey (6), J. C. White (2), (1) Connecticut Agric. Exp. Stn, New Haven, CT, USA; (2) Connecticut Agric Exp Station, New Haven, CT, USA; (3) University of Parma, Parma, ITALY; (4) The Connecticut Agricultural Experiment Station, New Haven, CT, USA; (5) International Fertilizer Development Center (IFDC), Muscle Shoals, AL, USA; (6) The University of Texas at El Paso, El Paso, TX, USA

Soil-borne pathogens cause over $20 \%$ losses in crop yields per year. Mineral elements, such as $\mathrm{Cu}, \mathrm{Mn}$, and $\mathrm{Zn}$, can activate enzymes to initiate defense mechanisms against disease. However, these plant nutrients are often not readily available at the root level in sufficient quantities to fight fungal infection. An alternate method to fungicides and other disease management approaches is to supply plants with nutrients of a nano-size that may be basipetally translocated. Metal oxide nanoparticles were sprayed at $500-1,000 \mu \mathrm{g} / \mathrm{ml}$ onto tomato, eggplant, and watermelon leaves. Plants were then grown in potting soils infested with Fusarium or Verticillium pathogens in the field and greenhouse. In the greenhouse, disease progress due to Fusarium (AUDPC) was reduced by $31 \%, 28 \%$, or $28 \%$ in tomato plants treated with nanoparticles ( $\mathrm{NPs}$ ) of $\mathrm{CuO}, \mathrm{Mn}_{2} \mathrm{O}_{3}$, or $\mathrm{ZnO}$, respectively. In eggplants, CuO NPs increased the fresh weight by $64 \%$, reduced the verticillium wilt AUDPC by $69 \%$ and the amount of $\mathrm{Cu}$ in roots incremented by $32 \%$. In the field, treatment with $\mathrm{CuO}$ NPs and bulk-size $\mathrm{Mn}_{2} \mathrm{O}_{3}$ increased the tomato yield by $\sim 30 \%$; while the eggplant yield was increased $34 \%$ and $41 \%$ in plants treated with $\mathrm{CuO} \mathrm{NPs}$ and $\mathrm{ZnSO}_{4}$ salt. Watermelon plants treated with $\mathrm{CuO}$ NPs had between $39-53 \%$ increases in yield. These findings show that supplying nano-nutrients though foliar application can potentially reduce root diseases and increase yield.

\section{The World Federation on Culture Collections: Promoting microbial utilization for over 70 years} K. MCCLUSKEY, Kansas State University, Manhattan, KS, USA

Research and application in plant pathology benefits greatly from access to validated microbial genetic resources. These may take the form of microbial type strains, or serogroup, vegetative compatibly group, or mating type testers as well as classical and GMO mutants, plant pathogen or growth promoting microbes, or whole genome sequence reference strains. To promote research robustness and reproducibility, validated microbial genetic resources are an essential infrastructure element for progress in plant pathology. The World Federation for Culture Collections (WFCC) is a multidisciplinary federation within the International Union of Microbiological Societies. It provides a forum for interaction among over 210 member collections and over 700 registered collections. WFCC member collections manage over 400,000 microbe strains from nearly 50,000 species. Registered collections hold over 2 million validated strains of fungi, bacteria, archaea and viruses. WFCC collections agree to implement collective best practices including quality control, data management, and regulatory compliance. Because plant pathogenic microorganisms are often subject to formal oversight, deposit of materials in public service collections is the best way to insure that exchange is carried out in compliance with national and international regulations. Deposit in WFCC registered collections also insures the greatest impact for publications utilizing microbial genetic resources and is required for patent and taxonomic purposes.

Cucumber green mottle mosaic virus: Research perspective working with a world travelling virus T. PITMAN (1), T. Tian (2), B. J. Aegerter (3), K. Posis (4), B. W. Falk (1), (1) University of California, Davis, CA, USA; (2) California Dept of Food \& Agric, Sacramento, CA, USA; (3) Univ. of California Cooperative Extension, Stockton, CA, USA; (4) California Department of Food and Agriculture, Sacramento, CA, USA

Cucumber green mottle mosaic virus (CGMMV) is a Tobamovirus that infects cucurbit crops and can cause devastating yield losses. CGMMV is primarily spread by contaminated seeds, then transmitted efficiently between plants by mechanical means. This allows geographical movement with the increased international seed trade, then rapid spread into naïve production areas. Originally described in England, global dissemination was slow until 2007, when detections in new regions increased rapidly. In California, the first detection of CGMMV was in 2013, after which annual detections occurred in either field settings or seed shipments. Our research into the genetic variability of CGMMV isolates detected in California indicates significant diversity and new introductions annually. Using Next Generation Sequencing, we have the full genomes of seven California introductions, two of which were identical. Additionally, we sequenced thirty-three isolates originating from Europe and southeast Asia. Incidentally, we identified a new cucurbit-infecting Tobamovirus to California in 2017, which was detected in material presumed to be infected with CGMMV. This virus shares $82 \%$ nucleotide identity with Cucumber mottle virus $(\mathrm{CuMoV})$, which was first described in 2006 from Japan. With increasing movement of seeds across international borders, ongoing monitoring is necessary to prevent plant diseases from becoming established in new regions.

Insights from abroad: Managing turfgrass diseases in Asia with minimal input M. WOODS, PH.D., Asian Turfgrass Center, Wanchai, HONG KONG

The most important factor for managing turfgrass diseases in Asia is the choice of turfgrass species. Tropical carpetgrass (Axonopus compressus) when grown in tropical regions of Asia almost never shows disease symptoms. All other turfgrass species used in Asia have some amount of disease, but the species differ in the amount of disease. Seashore paspalum (Paspalum vaginatum) in Southeast Asia is especially susceptible to multiple fungal pathogens. In the transition zone, disease symptoms are minimized by using Zoysia species. Control is required for large patch (Rhizoctonia solani), and fungicides are typically applied in spring and autumn to prevent disease symptoms. Manilagrass (Zoysia matrella) is susceptible to dog's footprint (Curvularia spp.) during warm and humid weather; fungicides are only applied to control this disease in high maintenance turf. Cool-season grasses are affected by the same diseases as seen at similar latitudes in temperate regions of the world. Annual bluegrass (Poa annua) is a common weed, but is rarely used as a maintained turf, so the diseases of that species are not as much a concern as they are in other parts of the world. My work has focused on supplying enough nutrients and water to the turf to produce a desired growth rate. This approach to turfgrass management results in minimal inputs and has some effect on turfgrass diseases.

Re-inventory of Australia's plant pathogen reference collections: Australian Colletotrichum species revisited J. EDWARDS (1,2), R. Shivas (3), Y. P. Tan (4), S. Q. Dinh (5), B. Weir (6), (1) AgriBio, Bundoora, AUSTRALIA; (2) LaTrobe University, Bundoora, AUSTRALIA; (3) Centre for Crop Health, University of Southern Queensland, Toowoomba, AUSTRALIA; (4) Department of Agriculture \& Fisheries, Brisbane, AUSTRALIA; (5) Agriculture Victoria, Knoxfield VIC, AUSTRALIA; (6) Manaaki Whenua Landcare Reseach, Auckland, NEW ZEALAND

In Australia, the effectiveness of biosecurity measures relies heavily on the accuracy of specimen-based databases of plant pathogens. Pathogen incidence data is essential for 'evidence of absence' that underpins market access and biosecurity for Australian produce, and plant pathogen reference collections and associated databases provide this evidence. With recent advances in fungal taxonomic research, identification of specimens based on the phenotype has been demonstrated to be inadequate and unreliable. This has enormous implications for quarantine and biosecurity, which rely on accurate diagnostic methods and accurate names. There is an urgent need for re-examination of herbarium specimens and living cultures using DNA-based methodologies. Colletotrichum is recognised as one of the most important genera of plant pathogenic fungi worldwide. In 2015, living cultures of 86 
specimens were sourced from Australian plant pathogen reference collections plus published DNA sequence data from a further 106 Australian specimens were found. Based on unambiguous DNA sequence matches to data from ex-type specimens, 44 species of Colletotrichum were confirmed as being present in Australia.

Identification of a novel target of the bacterial effector HopZ1a

P. ALBERS (1), S. Uestuen (1), K. Witzel (1), F. Bornke (1,2), (1) Leibniz-Institute of Vegetable and Ornamental Crops (IGZ), Grossbeeren, GERMANY; (2) University of Potsdam, Potsdam, GERMANY

The plant pathogen Pseudomonas syringae is a gram-negative bacterium which infects a wide range of plant species including important crops plants. To suppress plant immunity and cause disease $P$. syringae injects type-III effector proteins (T3Es) into the plant cell cytosol. In this study, we identified a novel target of the well characterized bacterial T3E HopZ1a. HopZ1a is an acetyltransferase that was shown to disrupt vesicle transport during innate immunity by acetylating tubulin. Using a yeast-two-hybrid screen approach, we identified a REMORIN (REM) protein from tobacco as a novel HopZ1a target. HopZ1a interacts with REM at the plasma membrane (PM) as shown by split-YFP experiments. Interestingly, we found that PBS1, a well-known kinase involved in plant immunity also interacts with REM in pull-down assays, and at the PM as shown by BiFC. Furthermore, we confirmed that REM is phosphorylated by PBS1 in vitro. Overexpression of REM provokes the upregulation of defense genes and leads to disease-like phenotypes pointing to a role of REM in plant immune signaling. Further protein-protein interaction studies reveal novel REM binding partners with a possible role in plant immune signaling. Thus, REM might act as an assembly hub for an immune signaling complex targeted by HopZ1a. Taken together, this is the first report describing that a REM protein is targeted by a bacterial effector. How HopZ1a might mechanistically manipulate the plant immune system through interfering with REM function will be discussed.

Plant disease detection utilizing artificial intelligence and remote sensing

Y. Ampatzidis (1), A. CRUZ (2), (1) University of Florida, SWFREC, Immokalee, FL, USA; (2) California State University, Bakersfield, Bakersfield, CA, USA

The rapid development of new technologies and the changing landscape of the online world (e.g., Internet of Things - IoT, cloud-based solutions) provide a unique opportunity for developing intelligent agricultural systems for precision applications. Technological advances in computer vision, mechatronics, artificial intelligence and machine learning have enabled the development and implementation of remote sensing technologies for plant symptom identification and disease management. These technologies can be used to distinguish between a variety of diseases with similar symptoms, and reduce diagnosis time and cost. Herein, a vision-based program to detect symptoms of several diseases from leaf images is presented. This system utilizes a deep learning convolutional neural network (DP-CNN) and a novel abstraction-level data fusion algorithm to improve detection accuracy. It can automatically detect plant diseases and discriminate from other disorders or pathogens, despite the strong similarity. For example, the program detects symptoms of Olive Quick Decline Syndrome (OQDS) on leaves of Olea europaea L. infected by Xylella fastidiosa with a true positive rate of

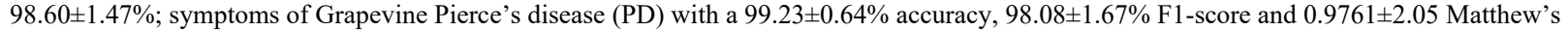
correlation coefficient; and symptoms of Grapevine Yellows (GY) with $92.0 \%$ accuracy and a Matthew's correlation coefficient of 0.832 .

Mycotoxigenic Fusarium spp. associated with stink bugs collected from corn fields in the mid-Atlantic U.S.

J. OPOKU (1), N. M. Kleczewski (2), K. Hamby (3), A. Coomber (4), D. Haak (5), H. L. Mehl (6), (1) Virginia Tech TAREC, Suffolk, VA, USA; (2) University of Illinois, Urbana, IL, USA; (3) Department of Entomology, University of Maryland, College Park, MD, USA; (4) Cornell University, Ithaca, NY, USA; (5) Virginia Tech, Blacksburg, VA, USA; (6) Virginia Tech Tidewater AREC, Suffolk, VA, USA

Stink bugs, including invasive brown marmorated stink bug (BMSB) (Halyomorpha halys), can severely damage crops including field corn. A survey of BMSB-infested corn fields in Virginia in 2013 identified a correlation between BMSB damage and fumonisin contamination. In artificially BMSBinfested and Fusarium inoculated field trials conducted in 2015, the ability of BMSB to increase Fusarium infection and fumonisin contamination in corn was demonstrated. Feeding damage is likely to contribute to increased infection and mycotoxin contamination, but the potential for stink bugs to vector Fusarium spp. has not been explored. In 2016, BMSB and native stink bugs were collected from corn fields in DE, MD, and VA to determine if mycotoxin producing Fusarium spp. could be isolated from their internal or external body parts. Fusarium was isolated from a total of 167 stink bugs collected from 8 corn fields. Partial RPB1 gene of each isolate was sequenced to identify species, and simple sequence repeat (SSR) markers were used to genotype isolates. Fumonisin-producing $F$. proliferatum and $F$. verticillioides and deoxynivalenol-producing $F$. graminearum were isolated from stink bugs. Within each species, a diversity of SSR haplotypes were identified. Mycotoxigenic Fusarium species are associated with stink bugs, so the insects have the potential to move these fungi across landscapes and vector the pathogens to susceptible crops including field corn.

Advances and innovative concepts to control Xylella fastidiosa colonization in citrus plants

A. A. DE SOUZA (1), M. Takita (2), M. Machado (2), R. Caserta (3), L. Mitre (2,4), L. Gómez-Krapp (2), C. Nascimento (2), D. Rebelatto (2), N. Safady (2,5), S. Picchi (2), H. Coletta-Filho (2), (1) Centro de Citricultura Sylvio Moreira - Agronomic Institute (IAC), Cordeiropolis, BRAZIL; (2) Centro de Citricultura Sylvio Moreira" - Agronomic Institute (IAC), Cordeiropolis, BRAZIL; (3) Centro de Citricultura Sylvio Moreira, Cordeirópolis, BRAZIL; (4) University of Campinas - UNICAMP, Campinas, BRAZIL; (5) Universidade Federal de São Carlos, Araras, BRAZIL

Xylella fastidiosa is responsible for diseases that affect different crops worldwide. One of them is Citrus Variegated Chlorosis (CVC), which results from the xylem vessels occlusion by $X$. fastidiosa biofilm. We have been working on two different approaches to control X. fastidiosa: i. transgenic plants and ii. N-acetylcysteine (NAC). For transgenic plants we focus in understanding the $X$. fastidios $a$ - plant host interaction to find key genes from both sides that could be used to break this interaction. Overall we are using genes from pathogen and from resistant host to transform susceptible varieties. One gene from the pathogen is $r p f F$, involved with $X$. fastidiosa quorum sensing. Transgenic plants carrying $r p f F$ showed high level of CVC resistance in Hamlin and Pineapple sweet orange varieties. On the plant side, we have identified genes associated with $X$. fastidiosa resistance, such as LRRRK/Pattern Recognition Receptors and a gene involved with methyl salicylate production. We have verified that transgenic lines expressing such genes were able to activate ROS response, increase the expression of defense related-genes and decrease $X$. fastidiosa colonization. The second approach is the use of NAC. In previous results we have demonstrated that this molecule shows effectiveness as an antimicrobial compound and biofilm disruption in $X$. fastidiosa. Here, we show advances in the study of the NAC effect in plants. Two years of evaluation in field where NAC was applied in plants showing severe CVC symptoms, revealed that application of NAC was able to improve fruit size in both diseased and healthy plants, and increased fruit production in healthy plants. Overall we will present new insights where the knowledge of plant-pathogen interaction can be used to obtain plant pathogen resistance. 
A mathematical framework for improving targeting of surveillance in complex pathosystems

A. MASTIN (1), F. Van den Bosch (2), T. R. Gottwald (3), S. R. Parnell (1), (1) University of Salford, Salford, UNITED KINGDOM; (2) Rothamsted Research, Harpenden, ENGLAND; (3) USDA-ARS, Ft Pierce, FL, USA

Emerging plant pathogens are an increasing source of economic loss and a risk to food security worldwide, and early detection surveillance is a key method of preventing their establishment. Our previous work has demonstrated the importance of accounting for the ecology and epidemiology of the pathosystem in question when planning surveillance, but inherent heterogeneities within natural pathosystems can make this challenging. One way to consider these heterogeneities is by dividing the system into distinct epidemiological groups, such as plant hosts and insect vectors, between which a pathogen spreads. Using this approach, we can create a mathematical model of pathogen transmission and, by linking it to a statistical model of a sampling process, derive a simple heuristic for estimating the incidence of infection at the time of first detection for any amount of sampling effort from the constituent groups. We demonstrate the application of this heuristic by considering the vector-borne causative agent of the citrus disease huanglongbing, and find that it performs well in comparison to computer simulation. Through this process, we have also found that targeting surveillance efforts towards either hosts or vectors, rather than both, can maximise the cost effectiveness of surveillance, with the optimal group to sample being influenced by the transmission rates between the groups and the relative sampling costs.

The impacts of global trade on the dispersal of fungal trunk pathogens in nursery stock D. GRAMAJE PEREZ (1), J. Armengol (2), R. Billones-Baaijens (3), F. Halleen (4), S. Di Marco (5), C. Rego (6), M. R. Sosnowski (7), J. R. UrbezTorres (8), (1) Instituto de Ciencias de la Vid y el Vino (ICVV), Logroño, SPAIN; (2) Univ Politecnica De Valencia (Q4618002B), Valencia, SPAIN; (3) National Wine and Grape Industry Centre, Wagga Wagga, AUSTRALIA; (4) Stellenbosch University, Stellenbosch, SOUTH AFRICA; (5) IBIMET - CNR, Bologna, ITALY; (6) Instituto Superior de Agronomia, LEAF, Universtiy of Lisbon, Lisbon, PORTUGAL; (7) University of Adelaide, Adelaide, AUSTRALIA; (8) Agriculture and Agri-Food Canada - Summerland Research and Development Centre, Summerland, BC, CANADA

An increasing amount of grapevine plant material, nursery plants and breeding stock, is moving vast distances, resulting in an increased risk of pathogen introduction into new areas. One of the primary concerns of the grapevine nursery industry is a group of fungal trunk pathogens for which there are no effective remedies once planting material is infected. A sizeable but unknown percentage of existing grapevine nursery stock produced under conventional nursery practices is likely to be infected with a broad range of taxonomically unrelated pathogens associated with grapevine trunk diseases (GTDs). These infections can eventually debilitate and kill infected plants after they are planted in the vineyard. Production practices in nurseries provide many opportunities for infection, through poor sanitation practices or introducing infected asymptomatic cuttings from mother vines. Infected plants may initially have no visible symptoms, but they may become apparent after a certain period of time when exposed to field stresses and depending on the level of infection. Detection and identification of these pathogens is challenging, as it requires destructive sampling from different plant parts due to their uneven distribution within the host. These factors represent a challenge for developing a proper clean plant program for GTDs in nurseries. Further research is needed to estimate the impacts of global trade on the dispersal of fungal trunk pathogens in other crops.

EDNA-Wheat, A Massive Parallel Sequencing Based Tool for Detection of Wheat Viruses

P. Rydzak, F. Ochoa Corona, A. C. WAYADANDE, Oklahoma State University, Stillwater, OK, USA

Each year, wheat growers lose significant yields to arthropod transmitted viruses and accurate diagnosis is essential for management. Electronic probe diagnostic nucleic acid analysis (EDNA) is a next generation sequencing (NGS) based diagnostic tool where highly curated, target-specific electronic probes (e-probes), 30-120 nucleotides in length, are used to query unassembled metagenomic databases for detecting the presence of target nucleic acid sequences. E-probes, 30 nucleotides in length and specific to 17 wheat infecting viruses, including three strains of Barley yellow dwarf virus (BYDVMAV, -PAV and -PAS) were designed, curated and validated in mock metagenomic databases generated using MetaSim and metatranscriptomic data obtained from healthy wheat tissue. To predict the sensitivity of EDNA as a diagnostic tool for the detection of viruses in wheat metagenomic data, two viruses, Wheat streak mosaic virus (WSMV) and BYDV, were selected as models to assess detection limits. Detection of all 17 viral pathogens of wheat and discrimination between three strains of BYDV was demonstrated in both single and multiple mock, in-silico infections. Thirty-five mock, in-silico metagenomic databases were generated with a known number of virus reads for WSMV and three strains of BYDV. It was determined that EDNA could detect a minimum of 31 virus reads out of a total of 10 million reads $(0.00031 \%)(\mathrm{p}=0.04332)$.

Agroecological engineering for biocontrol of soil pests - examples from the French Caribbean M. CHAVE, V. Angeon, INRA, Petit-Bourg, GUADELOUPE

Current crop protection based on elite varieties and intensive use of pesticides suffers from major limitations: emergence of pathogen resistance, alteration of natural resources, loss of biodiversity, risk to human health and dependence of farmers on external inputs. By promoting natural regulations, agroecological engineering is an alternative approach. It no longer offers one-stop solutions but calls for a set of biodiversity-based solutions that leave room for farmer initiatives. To illustrate this type of approach, the example arbuscular mycorrhizal symbioses are of growing interest in tropical areas facing strong challenging diseases. A participatory innovative design method involved farmers, researchers and technical advisers to identify potential solutions and combinations on how to enhance and benefit from mycorrhiza for crop health. Our main findings are that i) farmers propose a diversity of solutions related to the three steps of mycorrhizal fungi-plants interactions: fungal introduction in the field, establishment of symbioses and mycorrhizal network development, ii) propositions by farmers aimed at enhancing indigenous mycorrhizal networks rather than introducing exogenous propagules, iii) on farm innovative devices tested by farmers are efficient. Smallholder farmers are thus drivers in designing agroecological technologies. We will present how these findings are deployed as methods for teaching in agricultural school and for training farmers.

Cytokinin-mediated processes promote heat-induced disease susceptibility of Arabidopsis to Pseudomonas syringae pv. tomato A. M. SHIGENAGA, S. Johns, D. Bush, C. Argueso, Colorado State University, Fort Collins, CO, USA

Under increased temperatures, such as those predicted as a result of global climate change, plant defense responses are attenuated leading to a process known as heat-induced disease susceptibility. The plant growth hormone cytokinin regulates responses to both biotic and abiotic pressures. To address the role of cytokinin in heat-induced susceptibility of Arabidopsis to the bacterial pathogen Pseudomonas syringae pv. tomato (Pst), wild-type plants and a cytokinin receptor mutant (ahk2,3 mutated on ARABIDOPSIS HISTIDINE KINASE 2 and 3) were exposed to the pathogen at two different temperatures, normal $\left(22^{\circ} \mathrm{C}\right)$ and high $\left(28^{\circ} \mathrm{C}\right)$. Pst populations were measured to assess pathogen fitness and host susceptibility. Stomatal conductance and gene expression were also measured to evaluate how lack of cytokinin signaling impacts the ability of plants to respond to simultaneous biotic and abiotic pressures. We found that $a h k 2,3$ plants were less susceptible at $28^{\circ} \mathrm{C}$, with $P s t$ populations plateauing at 36 hours post inoculation in $a h k 2,3$. Moreover, pathogen-induced stomatal closure was impaired under heat stress and expression of genes associated with defense or nutrient transport were 
also affected. This suggests that cytokinin promotes physiological conditions that contribute to pathogen proliferation and highlights the value of cytokinin-based chemical and genetic approaches to understand plant susceptibility and improve crop protection under increased temperatures.

\section{Development of Nursery Plant Protection Strategies Based on Natural Products}

M. ABUGRAIN (1), M. Putnam (2), J. Chang (2), T. Mahmud (3), (1) Oregon state university, Corvallis, OR, USA; (2) Oregon State University, Botany and Plant Pathology, Corvallis, OR, USA; (3) College of Pharmacy, Oregon State University, Corvallis, OR, USA

Bioactive natural products have been known to be an important source of human medicines, e.g., antibiotics, anticancer, immunosuppressive agents, antiparasitics, hypolipidimic agents and antimalarials. Many clinically useful drugs have either been natural products, natural product derivatives, or synthetic compounds inspired by natural products. Some natural products have also been used to treat plant diseases. There are many plant diseases that have a significant economic impact. For instance, crown gall and leafy gall diseases caused by the bacterial pathogens, Agrobacterium tumefaciens and phytopathogenic Rhodococcus, respectively, have become a permanent threat in nursery and ornamental industry. Losses due to A. tumefaciens have amounted to many millions of dollars annually in the U.S. The disease caused by Rhodococcus can be responsible for up to $100 \%$ losses in some ornamental crops. However, there are no universally effective preventive and curative measures for infected plants. To this end, we embarked a program to test and develop control compounds for broad host range and gall forming bacterial pathogens based on plant and microbial natural products. Through bioassay-guided fractionation, we were able to isolate and characterize the chemical structures of active principles of two plant extracts with excellent antimicrobial activity against pathogenic Rhodococcus strains. Furthermore, we have isolated and structurally elucidated bioactive compounds from a bacterial isolate with significant antimicrobial activity against both Rhodococcus and Agrobacterium strains. Results of this study may lead to the development of new drugs for the prevention and/or treatment of crown gall and leafy gall diseases in nursery and ornamental plants.

Merging foundational and field research: Lessons from the ancient and emerging blast diseases on rice and wheat

B. VALENT (1), E. Oliveira Garcia (1), M. Dalby (1), M. Navia-Urrutia (1), C. D. Cruz (2), G. Cruppe (1), S. Liu (1), H. N. Trick (1), M. L. Farman (3), (1) Kansas State University, Manhattan, KS, USA; (2) Purdue University, West Lafayette, IN, USA; (3) University of Kentucky, Lexington, KY, USA

Rice blast disease, caused by the Oryza pathotype (MoO) of Magnaporthe oryzae (synonym of Pyricularia oryzae), continues to threaten global rice production. At the same time, rice blast has become a model for understanding the molecular mechanisms of fungal pathogenicity and host specificity. This hemibiotrophic fungus repeatedly invades living rice cells as it spreads within rice tissue for the first 4 to 5 days before macroscopic symptoms appear. $\mathrm{MoO}$ biotrophic rice cell invasion requires hyphal dimorphism, a specialized interfacial structure (the Biotrophic Interfacial Complex, BIC) and specialized mechanisms for delivering effector proteins inside living rice cells. Since 1985, wheat blast disease, caused by the Triticum pathotype (MoT) of $M$. oryzae, emerged as an explosive new disease in S. America and S. Asia. Wheat blast differs in key aspects of its field biology relative to rice blast. For example, wheat head blast and not foliar blast is the major disease symptom in the field, and resistance appears rare in wheat relative to resistance in rice. This raised the question of whether the MoT fungus undergoes the same biotrophic invasion strategy as MoO strains. We report that MoO and MoT have conserved effectors, and they share common effector dynamics and biotrophic invasion strategies. We report new understanding of the mechanisms by which blast effectors are translocated inside living rice cells, which will ultimately impact wheat blast control as well.

Culturable microbiota and metagenome data show distinct microbial assemblage between bacterial wilt disease-suppressive and conducive soils R. GICANA (1), W. L. Deng (2), (1) National Chung Hsing University, Taichung, TAIWAN; (2) Department of Plant Pathology, National Chung Hsing University, Taichung, TAIWAN

Suppressive soil is one the most interesting examples of community interaction that depict the role of the microbiome in influencing plant fitness towards specific diseases and offer microbiome-mediated protection of crops against soil-borne pathogens. Using high throughput amplicon sequencing of the 16S rRNA gene, we compared the culturable microbioata and the metagenome of the bacterial wilt disease-suppressive and -conducive soils identified from several eggplant farms in southern Taiwan. The culturable microbiota showed the abundance of the phylum Firmicutes for both soil phenotypes, and the predominance of the bacterium Bacillus fumarioli in the suppressive soil. The same result was obtained from the metagenomics analysis, which is contrary to the published results indicating the predominance of the phylum Proteobacteria in the soil. Regardless of the source of soil samples, the bacterial wilt disease-suppressive soil was dominated by B. fumarioli. Analysis of the community structure of the two soil phenotypes showed a higher alpha diversity in the conducive compared to the suppressive soil. Non-metric Multidimensional Scaling (NMDS), Principal Coordinates Analysis $(\mathrm{PCOA})$ and Hierarchical Cluster analysis showed significant clustering of the bacterial wilt disease-suppressive from the -conducive soil, indicating distinct microbial assemblage of these two soil phenotypes.

Upscaling models, downscaling data or the right model for the right scale of application?

A. H. SPARKS (1), K. A. Garrett (2), C. A. Gilligan (3), A. Nelson (4), K. Pembleton (1), (1) University of Southern Queensland, Toowoomba, AUSTRALIA; (2) Plant Pathology Department, University of Florida, Gainesville, FL, USA; (3) University of Cambridge, Cambridge, UNITED KINGDOM; (4) University of Twente, Enschede, NETHERLANDS

Plant epidemiological models are used in a range of applications, from detailed simulation models that closely follow pathogen infection and dispersal, to generic template-based models for rapid assessment of invasive species. There is increasing interest in applying small scale models - e.g., based on tissue, organ or whole plants - using remotely collected daily data, to generate regional risk information (e.g., maps). The assumption made is that such small scale models "scale-up" appropriately to regional, continental or even global scale. However, these models are often constructed using locally collected, hourly data. By necessity data available are often at much coarser scale, both temporally and spatially, than the data used to develop the model. Computational requirements increase considerably when more detailed models that require fine resolution data (if available) are applied to large areas, while small scale models often add little useful information at these scales and may lead to error propagation. Ideally, detailed models should be used at small temporal and spatial scales and less detailed models used for larger temporal and spatial scales. This paper presents examples of different approaches for changing scales - including upscaling models, downscaling data, and developing new models - and the issues that these approaches create or solve, along with ideas about how we can ensure that the scale of model and data match the desired application.

Aureobasidium pullulans suppression of green mould (Penicillium digitatum) development in mandarin var. 'Kinnow' through multiple modes of action

N. ADIKARAM (1), D. Singh (2), L. Jayasinghe (1), (1) National Institute of Fundamental Studies, Kandy, SRI LANKA; (2) ICAR-Indian Agricultural Research Institute, New Delhi, INDIA 
The objective of the study was to develop an effective biocontrol agent with multiple modes of action against green mould (Penicillium digitatum) in mandarin (Citrus reticulata var. 'Kinnow'). Aureobasidium pullulans was isolated from the mandarin fruit surface. Freshly harvested mandarins were treated with a cell suspension of different $A$. pullulans isolates and two days later, the treated and control fruits were inoculated with $P$. digitatum.

Treatment with $A$. pullulans Isolate Y 3 significantly $(\mathrm{p}<0.05)$ lowered green mould incidence compared to controls and other $A$. pullulans isolates tested. Dual culture of $P$. digitatum with Y3 on PDA slowed down colony growth indicating direct inhibitory activity of Y3. SEM studies revealed that Y3 cells had become embedded in the cuticle within 2-3 days of treatment. The cuticle showed signs of digestion, depressions underneath the yeast cells and shredding-off probably due to internal pressure exerted by embedded yeast cells. Some yeast cells proliferated producing buds and others made occasional physical contact with conidia of $P$. digitatum. Treated fruit peel displayed slightly greater antifungal activity than controls on TLC bioassay. Y3 was shown to produce non-specific esterases in cutin-amended medium. Yeast cells embedded might have physically obstructed $P$. digitatum entry and competed for space; cutin monomers produced by cutinase action triggered fruit resistance contributing to lowered green mould incidence.

The role of the Regional Plant Protection Organizations (RPPOs) in achieving the objectives of the International Plant Protection Convention (IPPC)

S. BLOEM, North American Plant Protection Organization (NAPPO), Raleigh, NC, USA

The International Plant Protection Convention (IPPC) is a multilateral treaty deposited with the Dir. General of the Food and Agriculture Organization in Rome, Italy. The purpose of the IPPC is international cooperation in controlling the introduction and spread of pests of plants and plant products and promoting appropriate measures for their control. The IPPC is administered by the Commission of Phytosanitary Measures (CPM) (currently 183 countries) in cooperation with regional and national plant protection organizations (RPPOs and NPPOs). The CPM meets once per year to discuss the state of plant protection in the world and to review and adopt international plant health standards. To date, 41 international plant health standards have been adopted by CPM. Regional plant protection organizations are named in Article IX of the IPPC; Art. IX.4 indicates that RPPOs should promote the development and use of international plant health standards and encourage inter-regional cooperation in controlling pests and preventing their introduction and spread. Currently, there are ten RPPOs; they get together once a year in a Technical Consultation where they discuss ways to cooperate in achieving the objectives of the Convention. Activities such as workshops on implementation of one of the international plant health standards, development of awareness materials about plant health and the exchange of emerging pest information are ways in which the RPPOs meet their objectives under the IPPC. This presentation will provide examples of activities and products developed by the North American Plant Protection Organization (NAPPO), the RPPO that represents Canada, the United States and Mexico, and how they have positively impacted adoption of international plant health standards and adherence to the Convention and its principles.

Understanding Maize chlorotic mottle virus transmission through seed: Localization and infectivity P. BERNARDO (1), M. G. Redinbaugh (1,2), K. Barriball (2), (1) Ohio State University, Wooster, OH, USA; (2) USDA, Wooster, OH, USA

Maize chlorotic mottle virus (MCMV) is the primary driver of Maize Lethal Necrosis, which has emerged recently in East Africa, Asia, and South America and has significant impact on maize production and food security. MCMV introduction to non-endemic areas is thought to occur through seed. To develop a better understanding of the mechanisms underlying MCMV transmission through seed, we investigated its distribution and infectivity in infested seeds from Kenya and Hawaii. MCMV was detected at high levels in the pericarp and pedicel in hand-dissected seeds using ELISA. Significantly lower levels of virus were detected in the endosperm and embryo, and no virus was detected in embryos that were washed after dissection. Subsequent immunofluorescence microscopy of seed sections indicated MCMV was localized to the pericarp and pedicel. Because no transmission of MCMV from infested seeds was detected in 1,800 seedlings, we tested the infectivity of virus isolated from these seeds. Although intact MCMV virions could be detected using transmission electron microscopy, and virus titer was sufficient for transmission of viable MCMV isolated from leaves, no transmission of MCMV from seed to uninfected maize could be achieved using leaf rub or vascular puncture inoculation. Our results indicate that MCMV from seed has low infectivity and is limited to maternal tissues, suggesting that seed treatments may reduce virus transmission through seed.

\section{Harmonization of phytosanitary/regulatory policy and seed health testing for safe global seed movement} S. THOMAS PHD, Monsanto, Creve Coeur, MO, USA

Many pests that are neither seed borne nor seed transmitted are regulated. Individual country seed import requirements differ substantially from one another for the same pest risk. The seed industry is a global business with international operations making it increasingly challenging to meet seed import requirements bilaterally and not multilaterally as befitting international trade. Seeds are a unique group of commodities where most pest risks can be collectively addressed by industry best practices reducing the need for commodity-specific phytosanitary requirements. ISF works to harmonize phytosanitary policy through the development of regulated pest lists, seed health tests, and encouraging recognition of industry best practices as alternative measures, or a systems approach, for import requirements. An extensive review of scientific literature by ISF for seed species with high volume trade shows that seed is a pathway for the introduction, spread and establishment of disease for a fraction of all regulated pests. Seed health testing is a tool used by the seed industry to prevent the introduction and spread of pests known to be associated with seed and ISHI-Veg, an industry-led group, develops internationally recognized seed health tests for seed-as-a-pathway pests. These tests are also part of the quality management practices companies use to protect their seed from exposure to pests and diseases at all stages of seed development, production and commercialization significantly reducing overall pest risk.

Evaluation of organic amendments to enhance dollar spot (Sclerotinia homoeocarpa) suppression on creeping bentgrass fairways C. BECKLEY, J. A. Roberts, University of Maryland, College Park, MD, USA

There is increasing interest in the use of alternative methods to standard fungicide programs for the control of dollar spot (Sclerotinia homoeocarpa F.T. Bennett) of turfgrass. Organic amendments, including composts and compost extracts, are one option that warrant additional examination. A 2-yr trial was initiated in late 2016 on creeping bentgrass (Agrostis stolonifera L.) maintained as a golf course fairway to evaluate various composts for dollar spot control when combined with fungicides. Main plot factor consisted of a municipal waste compost applied at establishment or as a bi-annual topdressing, a biochar applied at establishment or as a bi-annual topdressing, a standard fertility treatment, a standard fertility treatment + vermicompost extract program, or none. Sub-plot factor consisted of fungicides (contact $14 \mathrm{~d}$, penetrant $14 \mathrm{~d}$, contact threshold, penetrant threshold, or none). Threshold fungicide treatments were applied once two of four replicates exhibited $>2$ infection centers per plot. Fertility levels within all main plots were normalized over the trial period. In 2017, all fertility treatments significantly reduced dollar spot incidence compared to none on three of six individual rating dates. An ongoing analysis of nutrient content in leaf tissue and of soil microbes will aid our understanding of nutrient availability in addition to non-nutritional effects composts have on dollar spot development. 
New developments in the control of black Sigatoka and Fusarium wilt in banana

H. Sierotzki (1), S. TORRIANI (1), M. Guzman (2), A. Dutton (3), M. Oostendorp (4), (1) Syngenta Crop Protection, Stein, SWITZERLAND; (2) Syngenta LAN, S. A., Guatemala City, GUATEMALA; (3) Syngenta Crop Protection, Basel, SWITZERLAND; (4) Syngenta Crop Protection AG, Basel, SWITZERLAND

Banana production rely mainly on one clonal cultivar, Cavendish, which is world-wide grown and therefore highly prone to diseases. Major diseases are Mycosphaerella fijiensis and M. musicola, which are controlled by a wide range of fungicides coming from 10 different classes. The agronomic conditions in which bananas are cultivated demand frequent applications. In principal multi-site fungicides are used frequently and site specific systemic fungicides are added in critical phases of the disease epidemics. In many regions resistance to various fungicide classes has developed and needed to adapt the use recommendations. Resistance is found for BMC, QoI, DMI and SDHI (even if not widely spread) fungicides in many areas and therefore restrictive guidelines were agreed. Other fungicide classes are free from significant resistance evolutions, but precautious measures are agreed. Recently biologicals have been introduced into the spray program. An overview on the fungicide classes and associated resistance, geographical spread and known mechanisms will be given, describing the consequences for the use pattern of fungicides. Panama disease caused by Fusarium oxysporum f. sp. cubense TR4 race is recently re-occurring threat to many banana cultivars, including Cavendish. This race is spreading within Asia and endangering other major banana growing countries. Since no direct control measures are available, a range of indirect measures are in place to limit further spread. Research activities have been started to investigate the pathogen, the host resistance and direct control. A project together with the University of Wageningen has been initiated to elucidate the possible usage of fungicides towards Fusarium oxysporum f. sp. cubense.

Plant pathogenic and toxigenic Fusarium species - their taxonomy, systematics and nomenclature in the molecular age B. A. SUMMERELL, Royal Botanic Gardens and Domain Trust, Sydney, AUSTRALIA

The fungal genus Fusarium contains some of the most economically and socially important species of plant pathogens affecting agriculture and horticulture. It also contains numerous species that are important mycotoxin producers and some that are increasing in importance as pathogens of humans. Some of the plant diseases, such as head blight of wheat and Fusarium wilt of bananas, are amongst the most important diseases of these hosts and have not only caused enormous losses in production around the world but have also had a huge impact on the communities that depend on these crops. The genus is a complex, polyphyletic grouping whose taxonomy has always been controversial with species numbers ranging from over a 1000 at the beginning of the 1900s, down to 9 in the 1950s and '60s and currently anything from nearly 100 to 500 . The generic boundaries are also currently a point of debate. This talk will provide an overview of Fusarium, its phylogeny and biogeography and the mechanisms involved in speciation and the evolution of pathogenicity.

Towards deciphering host resistance to phytonematodes: Transcriptome analysis of a coffee incompatible response to Meloidogyne incognita P. Grynberg (1), A. S. Petitot (2), A. Mota (3), R. Togawa (1), D. Fernandez (2), E. V. S. ALBUQUERQUE (1), (1) Embrapa Recursos Geneticos e Biotecnologia, Brasilia, BRAZIL; (2) IRD, CIRAD, Univ Montpellier, IPME, Montpellier cedex 5, FRANCE; (3) Universidade do Rio Grande do Sul, Porto Alegre, BRAZIL

The root-knot nematode (RKN) Meloidogyne incognita is considered one of the most economically damaging pathogen in coffee crops. Breeding introgression strategies with $\mathrm{R}$ genes result in resistance breaking, while chemicals nematicides are considered harmful. We had previously observed that the development of M. incognita in Coffea arabica is prevented by the necrosis of the host cells at 6 days after infection (dpi) in (R) roots, while the feeding sites are being established by the formation of giant cells in (S). Samples from these contrasting materials were sequenced by RNAseq Illumina HiSeq 4000, generating over 800 million 2x100 nt length reads. Differential expression analysis and GO enrichment results point out that some gene families are strongly deregulated in the resistance response. These genes could be important players in the coffee-RKN incompatible interaction and may be potentially used in biotechnological approaches to nematode control.

Improved surveillance of diseases using nano-pore sequencing N. BOONHAM (1,2), I. Adams (1), A. Fox (1), J. Smith (1), (1) Fera Science Ltd, York, UNITED KINGDOM; (2) Newcastle University, Newcastle upon Tyne, UNITED KINGDOM

Next generation sequencing (NGS) has revolutionised plant pathogen diagnostics, as prices continue to fall and applications continue to expand. At the forefront of this revolution are the detection, diagnosis and characterisation of plant viruses. In 2011 a new disease emerged in maize production in Kenya. Whilst early attempts to provide a diagnosis using conventional methods failed, NGS was used to rapidly identify suspect viruses (Maize chlorotic mottle virus and Sugarcane mosaic virus) which were later confirmed using PCR testing, whilst mechanical inoculation satisfied Koch's postulate. The rapid identification of the causal agents of an emerging disease (maize lethal necrosis) enabled rapid development and deployment of qPCR tests in local labs enabling diagnosis of suspected outbreaks and seed testing. The genomes analysed from Kenya, South Sudan, Rwanda and Ethiopia shows that the isolates of MCMV recovered are highly conserved, whilst the sequence of ScMV is divergent and in some cases other potyviruses are present. This suggests that the spread of MCMV is driving the disease epidemic, whilst potyviruses are a frequent local infection. Follow-on testing suggests that in some locations the disease symptoms in the field are being confused with those caused by other viruses. The most commonly detected of these was the recently characterised 'maize yellow mosaic virus' at a number of locations. These data show the value of incorporating NGS into routine diagnostics, especially in outbreak situations. The use of nanopore sequencing (Oxford Nanopore) was explored which may be more suited to resource poor settings. The results show that despite the increased error rate, the technology can be used to provide valuable genome sequence data and cloud based analysis supports use by non-specialists and negates the need for elaborate IT infrastructure.

\section{Aldaulactone, a new phytotoxin involved in Alternaria dauci - Daucus carota interaction}

J. COURTIAL (1), L. Hamama (1), J. J. Helesbeux (1), M. Lecomte (2), Y. Renaux (1), E. Guichard (1), L. Voisine (1), C. Yovanopoulos (1), B. Hamon (1), L. Ogé (3), P. Richomme (1), M. Briard (3), T. Boureau (1), S. Gagné (1), P. A. Poupard (1), R. Berruyer (1), (1) University of Angers, Angers, FRANCE; (2) SUBA France, Nogaro, FRANCE; (3) Agrocampus Ouest, Angers, FRANCE

The mechanisms of quantitative aggressiveness and partial resistance have not been extensively studied and are prevalent in most plant - necrotrophic pathogen interactions, including the Daucus carota - Alternaria dauci interaction. In those, pathogens often produce phytotoxic secondary metabolites. In the work presented here, we explored the link between $A$. dauci aggressiveness and toxin production. In a first set of experiments, we uncovered a link between aggressiveness and fungal exudates toxicity on in vitro cultured carrot cells. These results were obtained with cells from the $A$. dauci susceptible genotype H1. Cells from the partially resistant genotypes K3 and I2 were only affected by exudates from the most aggressive $A$. dauci strain, ITA002. We made the hypothesis that toxicity of the fungal exudates was correlated with the amount of toxic compounds produced by the fungus. Interestingly, apolar fungal exudates production was correlated with strain aggressiveness. We analyzed the apolar fungal exudates using HPLC, and correlations 
between observed peaks intensity and fungal aggressiveness were measured. One of the observed peaks was strongly correlated with fungal aggressiveness. NMR analysis revealed that the purified compound was a novel polyketide with a decalactone ring. We named it "aldaulactone". In a new set of experiments, involving automated image analysis, we found that aldaulactone is toxic to in vitro cultured plant cells at concentrations observed in fungal exudates. Similarly, the effect of both aldaulactone and fungal extracts was weaker on I2 plant cells than on H1 plant cells. Taken together, these results suggest that (i) aldaulactone is a phytotoxin, (ii) variations in aldaulactone production affect fungal aggressiveness and (iii) resistance to aldaulactone is a mechanism of carrot resistance to $A$. dauci.

Efficient genome editing in Fusarium oxysporum based on CRISPR/Cas9 ribonucleoprotein (RNP) complexes Q. WANG, P. Cobine, J. Coleman, Auburn University, Auburn, AL, USA

The Fusarium oxysporum species complex (FOSC) is an important group of filamentous fungi that are able to cause disease on over 120 genera of plants. Based on its economic importance, $F$. oxysporum has become an important microbe for studying the genetics and pathogenicity of a plant pathogen; however, efficient reverse genetic methods including gene disruption/deletion strategies are limited. To aid in the study of these fungi, we developed a gene editing system using a $F$. oxysporum-optimized Cas9 RNP transformation method. The optimized Cas9 protein and sgRNA can be assembled to form a stable ribonucleotideprotein in vitro and this complex and/or template donor DNA can be transferred into fungal protoplasts for gene editing by PEG-mediated transformation. This procedure was developed using Cas9 RNPs that were transferred to fungal protoplasts to disrupt two genes (URA5 and URA3), generating uracil auxotroph mutants and are resistant to 5-FOA. Another gene, bik1, was used to further optimize this system where the maximum efficiency of disruption for this gene was able to reach $\sim 50 \%$. Further analysis of the bik1 mutant confirmed that this polyketide synthase was involved in the synthesis of the red pigment bikaverin. All mutants in this study demonstrated strong expected phenotypes, which suggests this F. oxysporum-optimized CRISPR/Cas9 system is stable and can be efficiently used to disrupt the gene(s) of interest.

Role of effector proteins in pathogenicity of postharvest pathogens

S. DROBY, Agricultural Research Organization, The Volcani Center, Rishon Lezion, ISRAEL

Penicillium expansum regarded as one of the most important postharvest rots of apple fruit and of great concern to fruit processing industries due to secretion of potent mycotoxins. Elucidating the pathogenicity mechanisms of this pathogen is of utmost importance for the development of effective and safe management strategies. We found that $P$. expansum secretes proteins during apple infection that suppress resistance related ROS production in the apple wounds. These findings suggest the possibility that effector-proteins may play an important role in the $P$. expansum interaction with the host. In the current study, bioinformatic tools were used to contruct a pipeline to predict potential effector genes in $P$. expansum and study their role in pathogenicity on apples. Features such as secretion, differential expression in planta during infection, small size, and large number of cysteines as well as information available in databases on known effectors were used to predict genes relevant to processes associated with the infection of apples by $P$. expansum. Applying the effector-prediction pipeline to the secretome of $P$. expansum revealed 103 enzymes that degrade cuticle-building polymers, carbohydrates, peptides, lipids and phospholipids, as well as 106 potential effector proteins. Among the predicted effectors that were identified, 47 of them have homology to known effectors. LysM proteins, NEP-1-like proteins, small-cystein rich (SCR) proteins with unknown function and subtilisin-related Peptidase S8 were characterized and functionally analyzed. We found several putative effectors that have an effect on the pathogenicity and virulence of P. expansum on apples as well as proteins with pleiotropic effect affecting growth rate, morphology and sporulation.

Associations of Armillaria root rot, Trichoderma endophytes and host plants in UK gardens

J. DRAKULIC (1), N. Bashir (2), M. Cromey (1), G. Clover (3), L. Beal (1), (1) Royal Horticultural Society, Woking, UNITED KINGDOM; (2) University of Nottingham, Nottingham, UNITED KINGDOM; (3) Royal Horticultural Society, London, UNITED KINGDOM

Armillaria root rot affects hundreds of plant species and can cause the death of mature trees in parks and gardens. Management advice for gardeners is to remove infected root systems and prevent below ground spread with impermeable barriers. This approach is costly, labour intensive, difficult to perform in most gardens, and thus often unsuccessful. Our three-year survey of UK gardens found that the vast majority (83.1\%) of plant deaths by Armillaria spp. are due to the aggressive pathogen A. mellea. Ligustrum was the most commonly reported host, highlighting the threat from Armillaria to hedges, and 14 new hosts were recorded that extend the already broad host range. Garden planting choices are greatly restricted if species susceptible to Armillaria root rot are avoided. Trichoderma endophytes were studied for their potential to improve host resilience and promote growth during establishment when root systems are at greatest risk of succumbing to Armillaria infection. Introduction of suitable isolates to susceptible plants would empower gardeners to replant Armillaria-infected beds. Susceptible hosts that had evaded disease from infection foci at RHS Garden Wisley were sampled. Endophytes from these root systems were cultured, identified and screened for beneficial effects on common garden plants (Rosmarinus officinalis and Thymus vulgaris). T. atrobrunneum was isolated most frequently, and has shown improved host growth and in vitro inhibition of $A$. mellea.

Resistance and resistance breaking mechanisms in the melon/Melon necrotic spot virus interaction V. Truniger, M. Miras, M. A. ARANDA, CEBAS-CSIC, Murcia, SPAIN

Melon necrotic spot virus (MNSV; genus Carmovirus, family Tombusviridae) is a pathogen endemic in cucurbit crops worldwide. In melon, the recessive $n s v$ gene confers immunity to MNSV. Resistant ( $n s v / n s v)$ melon hybrids have been developed and are widely cultivated. Nsv codes for the eukaryotic translation initiation factor (eIF) 4E, which together with the scaffolding protein eIF4G forms the eIF4F cap-binding complex. Although $n s v$ resistance seems to be durable, at least two resistance-breaking MNSV isolates have been described: MNSV-264 has the peculiar ability of infecting not only resistant melons, but also Nicotiana benthamiana and Gomphrena globosa plants, which are normally non-hosts for MNSV. MNSV-N infects resistant melons but not $N$. benthamiana or G. globosa plants. For both isolates, this capacity depends on the 3'untranslated region of the virus, which contains an RNA structural element that functions as cap-independent translation enhancer (CITE) needed for efficient translation of viral mRNAs. Avirulent MNSV isolates contain an I-shaped 3'-CITE of approximately 50 nucleotides that specifically interacts with eIF4E; thus, when critical nucleotide or amino acid substitutions are introduced in either partner, the interaction does not occur, abolishing translation of viral mRNAs and, consequently, virus multiplication. Virulent MNSV isolates harbor different 3'-CITEs that confer the virus the ability to translate its mRNAs in the presence of the resistance eIF4E allele or even in eIF4E absence. Interestingly, both virulent isolates have acquired their new 3'-CITEs by recombination, suggesting that 3'-CITEs are modular, interchangeable RNA elements with important adaptive significance.

Understanding the basis of host and non-host defences during barley-aphid interactions C. ESCUDERO-MARTINEZ (1,2), D. Leybourne (1,2), A. Barakate (1), J. Morris (1), P. Hedley (1), J. Stephens (1), J. Bos (1,2), (1) The James Hutton Institute, Invergowrie, UNITED KINGDOM; (2) University of Dundee, Dundee, UNITED KINGDOM 
Aphids are phloem-feeding insects that cause important yield losses on crops, including cereals such as barley. Most aphid species are limited to one or few host species, but some are able to reproduce on many plants belonging to different families. Interestingly, aphid probing-behaviour can be observed on both host and non-host plants indicating a requirement for molecular events to take place which may dictate the aphid host range. We found that the barley specialist Rhopalosiphum padi and the broad host range Myzus persicae showed strong differences in colonization, phenotype and probingbehaviour on barley. Preliminarily, the Electrical Penetration Graph (EPG) technique has shown that both aphids successfully penetrate the barley leaf surface; but differ in their ability to reach the phloem. EPG feeding patterns are being investigated to unravel the resistance source against $M$. persicae. Analyses of barley transcriptional responses revealed gene sets differentially regulated upon the different barley-aphid interactions, where $M$. persicae induced the strongest response. Interestingly, we identified several genes highly up-regulated upon $M$. persicae interaction, and to a lesser extent upon $R$. padi, including thionins and a late embryogenesis abundant gene. Ectopic expression of two barley thionins in Nicotiana benthamiana reduced host susceptibility to M. persicae, potentially reflecting a role in defence against aphids. We have generated barley knock-out lines, using CRISPR-Cas9 technology, for these genes to investigate their role in plant-aphid interactions. Our work thereby provides novel insights into host and non-host defences in a monocot crop against aphids and implicates thionins in the resistance to the global pest $M$. persicae.

Genetic tools for the study of light and circadian processes in microbial plant pathogens

L. E. CADLE-DAVIDSON, USDA Grape Genetics Research Unit, Geneva, NY, USA

With increased biological understanding of how hosts and pathogens sense and respond to light, genetic tools are needed to characterize the fundamental biology as well as to develop novel light-based strategies for disease management. These genetic tools, however, are not one size fits all, given the diversity of pathosystems studied and the diversity of applications considered. Here, as case studies, several approaches we have applied to powdery mildews will be discussed. These began with RNA-Seq and comparative genomic analysis to identify candidate genes. Population genetic analysis of AmpSeq data and population genomic analyses enabled the characterization of gene conservation, which could help predict evolutionary responses to light-based disease management. Mutants and other genetic resources on the host side help to untangle direct versus indirect (via the host) pathogen responses to light. Finally, without being able to axenically culture or stably transform the pathogen, functional studies in heterologous hosts may provide insights into the biological function of candidate genes, with the caveat that a gene may have different phenotypes in different genetic backgrounds. These and other genetic approaches may provide additional strategies for or improved implementation of light-based disease management.

\section{Genomic signatures of sub-lethal fungicide stress in Sclerotinia sclerotiorum}

\section{N. GAMBHIR, Z. N. Kamvar, S. E. Everhart, University of Nebraska, Lincoln, NE, USA}

Cellular stress from exposure to sub-lethal doses of antifungals is known to cause genomic instability in human pathogens, which may confer antifungal resistance or other adaptive traits, yet little is known about similar genomic stress on fungal plant pathogens. In a previous study, we exposed five isolates of Sclerotinia sclerotiorum to sub-lethal doses of four commercially formulated fungicides with different modes of action (iprodione, dicarboximide; thiophanate-methyl, MBC; boscalid, SDHI; azoxystrobin, QoI) for twelve consecutive generations with experimental replication. In the present study, we sequenced the genomes of pre- and post-exposure individuals (55 in total). Obtained were $115 \mathrm{~GB}$ of $150 \mathrm{bp}$ paired-end reads that were aligned to the reference genome and used to call variants, which were filtered for read depth ( $>5 \mathrm{X})$, mapping quality ( $>\mathrm{Q} 41)$, and loci that mutated in controls. From the first experiment, identified were 297 variants, $42 \%$ were SNPs and $22 \%$ variants were in a putative coding region. From the repeated experiment, results were similar: 294 variants, $50 \%$ SNPs and $25 \%$ variants in the coding region. This is the first study to characterize genomic alterations of a plant pathogen after exposure to sub-lethal fungicides. Further analysis will characterize duplications, chromosomal rearrangements and mutated genes under positive selection.

The future is now - a new technology for high-resolution aerial imagery G. SHARABANI, Taranis, Tel Aviv, ISRAEL

Several approaches for precision agriculture were developed to provide decision support system for whole farm management. However, the current technologies are not fast enough and lack high-resolution imagery. Taranis, a precision agriculture company, develops deep learning models based on data sets that include, field sensors, satellite imagery, weather forecast, disease models and data from the field scouting application. A new technology, $\mathrm{AI}^{2}$, has been developed by Taranis, which includes sub-millimeter aerial imagery and artificial intelligence. It provides high-resolution view of thousands of points in the monitored field for biotic and abiotic stress evaluations. It uses algorithm that can differentiates between damaged and health plants. $\mathrm{AI}^{2}$ technology is able to identify the very first symptom in the field. The technology achieves unparalleled scale and resolution to acquire quality images at the resolution of the human eye while covering far more land than the currently available traditional methods. For an example, in soybean field, the system covers 800 hectares in about 1 hour with more than 2,000 images, whereas the typical manual process by an agronomist covers this area in about four days. The advantages of this technology are early detection of the damaged plants and covering large area of the inspected field. In addition, it enabled to assess the efficacy of applied treatments and performing a return of inputs analysis for the grower in real time.

\section{Harmonization and standardization of pathogen strains for the global movement of seed} V. GRIMAULT, GEVES-SNES, Beaucouzé Cedex, FRANCE

Consistent identification of plant pathogen strains and races used for resistance tests is a recognized need for the global seed industry. Several initiatives have been launched to achieve consistent testing results. The Harmores projects were set up to harmonize test protocols and Reference Materials (susceptible and resistant controls, host differentials and isolates). The Plantum/Naktuinbouw isolate collection in Netherlands and the MATREF (MATerial of REFerence) network in France (2002) were established in collaboration with the seed industry for the distribution of isolates and seeds of controls and differentials. MATREF also conducts the appropriate phytosanitary and functional testing against pests before distributing. Material transfer agreements, letters of authorization and special permits are sent with the reference materials. The Harmorescoll initiative, based on the above services is being proposed to develop a unified European system for the distribution of Reference Materials. There was no system in the US, which resulted in differences in strain naming between the US and Europe. The Collaboration for Plant Pathogen Strain Identification (CPPSI) was initiated in 2007 to develop a US based industry standard for the identification of plant pathogen strains and races based on sets of differentials. At the world level, the ISF Working Group on Disease Resistance Testing, is centralizing information developed by these initiatives and coordinating actions for standardization of material and naming. The working group describes differentials sets. It has set up a harmonized codification of pests and is currently setting up a position paper on how to nominate new strains and races. Examples of these initiatives will be discussed on the collaborative harmonization to facilitate the global movement of cultures/seeds. 
Farmers' knowledge and management of potato late blight in Peruvian highlands: Implications for an integrated disease management program W. PEREZ (1), R. Arias (2), M. Barreto (2), K. Sanabria (1), J. L. Andrade-Piedra (3), (1) International Potato Center, Lima, PERU; (2) Private, Paucartambo, Pasco, PERU; (3) International Potato Center (CIP), Lima, PERU

Using a semi-structured questionnaire, 1198 Peruvian farmers were interviewed on their knowledge about late blight (LB) and its current management practices. Farmers overwhelmingly considered LB as the main foliar constraint in both potato growing areas. However, most farmers considered the interaction of a rainfall, sunny and foggy days as the cause of the disease and only few mentioned to a "fungi". The main method to control LB is the use of fungicides, but some of them use insecticides, foliar fertilizers, hormones, and commercial leach. Commonly, the first fungicide spray is at plant emergence and then applications between 7 and 8 days. Fungicides based on cymoxanil and mancozeb were the most used in both areas, however, farmers mix until 5 pesticides at the same time. Over dosage or under dosage are common among farmers, which follow indications of pesticide sellers or use their own criteria. Farmers overwhelmingly no distinguished different levels of susceptibility among Peruvian potato varieties. To improve farmers' capacities for better management of LB, is necessary to change pesticide-handling practices, promote the use of resistant varieties and use of other complementary control practices, through of an intensive, participatory training, which requires the support and involvement of key stakeholders, including agrochemicals companies, farmers, national and international R\&D organizations, donors, governments, and NGOs.

Producing high quality ornamental crops with limited chemical options: A Canadian perspective A. M. POLEATEWICH (1), S. Jandricic, Ph.D. (2), (1) University of New Hampshire, Durham, NH, USA; (2) Ontario Ministry of Food, Agriculture and Rural Affairs, Vineland Station, ON, CANADA

The use of integrated pest management strategies has been widely adopted among Canadian ornamental growers for management of pests and diseases A key driver is the high costs and limited number of pest control chemicals available to Canadian growers compared to their U.S. counterparts, which poses a competitive disadvantage. Strict regulations limit the number of new registrations and have prompted deregistration or limited use of key active ingredients. These challenges have increased research and adoption of other strategies to manage pests and diseases. The Canadian greenhouse industry has positioned itself as a leader in the implementation of biological control of insect pests with little to no chemical inputs. While innovation in disease management lags behind insect control, growers are finding success with IPM strategies, particularly with an emphasis on prevention. With limited curative options, sanitation and water treatment are essential to providing a solid foundation at the beginning of the production cycle. Cultural and biological strategies used in Canadian ornamental production will be discussed. A case study will also be presented to illustrate the value of prevention in producing high quality crops with little chemical input. In this study, the combination of sanitizing production surfaces, raising pots off the ground and application of biopesticides was as effective as a chemical program in terms of plant marketability and disease incidence.

\section{Managing fungicide resistance using the principles of population biology: Insights from mathematical modeling and field experiments} A. MIKABERIDZE, Epidemiology of Plant Diseases, ETH Zurich, Zurich, SWITZERLAND

To preserve efficacy of fungicides, we first need to understand the ecological and evolutionary principles that govern pathogen adaptation to fungicides. Fungicide resistance develops in two phases. First, resistant mutants arise through mutation and invade the pathogen population (emergence phase). After successful emergence, the frequency of resistance increases due to selection imposed by a fungicide in a deterministic fashion (selection phase). Fungicide dose can be adjusted to suppress emergence of resistance. To investigate this possibility, we combined a population dynamical model with dose-response field data for Zymoseptoria tritici, an important wheat pathogen. To reduce selection for resistance, one can optimize the proportion of fungicides in the mixture as our modeling study indicates. However, this requires that substantial fitness costs are associated with mutations conferring fungicide resistance. I will discuss further possibilities to suppress fungicide resistance by (i) optimizing spatial scales of fungicide applications and (ii) combining fungicides with host resistances. To find optimal strategies, we need to better characterize pathogen's epidemiological parameters such as infection efficiencies, transmission rates and dispersal kernels. This knowledge would inform modeling studies with the aim to propose best candidate strategies, which will need to be tested in the field. I will review our work in progress in this area.

Remote Sensing Technology for Early Detection of Root Decline in Putting Green Turfgrass

M. TUCKER (1), A. Fox (1), A. Badial (1), J. King (1), T. N. Spurlock (2), M. Tomaso-Peterson (1), (1) Mississippi State University, Mississippi State, MS, USA; (2) University of Arkansas, Monticello, AR, USA

Root decline is associated with ultradwarf bermudagrass (UDB) greens during the summer months in the southeastern United States. Abiotic factors coupled with a complex of fungal root pathogens from the genera Gaeumannomyces, Magnaporthiopsis, and the novel, Candidacolonium cynodontis contribute to this syndrome. Remote sensing technologies (RST) are commonly used in precision agriculture; however, management of amenity turfgrasses have not kept pace. The goal of this study is to demonstrate the application of RST for use in monitoring UDB for plant health and the distribution of root decline pathogens within putting greens. A fishnet grid system using ArcGIS was established for sampling $(\mathrm{n}=254)$ putting greens Aerification cores were collected within $2.4 \mathrm{~m}^{2}$ of each centroid. Composite root samples were analyzed for root health using WinRhizo Pro and fungal identification was based on multiplex qPCR assays. Normalized Difference Vegetation Index (NDVI) using a drone and visual turfgrass quality ratings were conducted when UDB was actively growing. To date, Magnaporthiopsis, C. cynodontis, and Gaeumannomyces spp. were detected in association with UDB roots at 55,15 , and $10 \%$, respectively. NDVI ratings (scale $=0-1 ; 1=$ highest reflectance) in those quadrats were $\leq 0.375$ and root health averaged $<10 \%$. Results of this research may serve as a model to integrate RST into turfgrass management for early disease detection and reduced fungicide inputs.

Transforming disease management through the use of unmanned aerial systems

J. VAN AARDT (1), E. Hughes (1), S. J. Pethybridge (2), J. Kikkert (3), C. Salvaggio (1), (1) Rochester Institute of Technology, Chester F. Carlson Center for Imaging Science, Rochester, NY, USA; (2) Cornell University, Plant Pathology \& Plant-Microbe Biology Section, Geneva, NY, USA; (3) Cornell Cooperative Extension, Canandaigua, NY, USA

Relatively recent advances in unmanned aerial systems (UAS) technology, as well as miniaturization of complex remote sensing systems, have enabled new approaches to precision agriculture. For example, imaging spectroscopy (hyperspectral) and light detection and ranging (lidar) can be used for moisture stress assessment, nutrient mapping, and disease detection. We specifically are developing risk models for proactive management of white mold (Sclerotinia sclerotiorum) in snap beans. Prophylactic fungicide applications, applied when $10 \%$ of plants have at least one bloom, typically are used to prevent the mold. The goals are to identify spectral signatures of blooming onset, investigate spectral characteristics of white mold onset in the snap bean crop, and assess 3D lidar point clouds for structural inputs to risk models. The study area is located at the New York State Agricultural Experiment Station, Geneva, NY, USA, operated by Cornell University. A DJI Matrice-600 UAS, boasting a high spatial resolution color camera, a Headwall Photonics imaging spectrometer (272 bands; 400-1000 nm), and a Velodyne VLP-16 lidar system, is being used for this research. Our analysis approach 
involves a series of high frequency flights - centered around blooming and white mold onset - throughout the growing season. We will present the initial findings of this work, focusing on the need for proper calibration-to-reflectance of the imaging spectroscopy data, identifying an operational set of wavelengths from this spectrally oversampled imagery, and the benefit of fusing 3D lidar data with high fidelity spectral data for inclusion in the risk modeling effort. This UAS sensing approach hopefully one day can become standard practice.

\section{Decision support systems for late blight control and early warning}

F. LUCCA (1), G. Kessel (2), W. E. Fry (3), I. Acuna (4), R. Bravo (4), W. Perez (5), J. L. Andrade-Piedra (6), J. Grønbech Hansen (7,8), P. Kromann (9), M. Guo (10), (1) Instituto Nacional de Tecnología Agropecuaria (INTA) (+TizonLatino Network), Balcarce, ARGENTINA; (2) Wageningen Plant Research, Wageningen, NETHERLANDS; (3) Cornell University, Ithaca, NY, USA; (4) Agricultural Research Institute INIA Chile, Osorno, CHILE; (5) International Potato Center, Lima, PERU; (6) International Potato Center (CIP), Lima, PERU; (7) Aarhus University, Tjele, DENMARK; (8) Aarhus University, Aarhus, DENMARK; (9) International Potato Center, Quito, ECUADOR; (10) Heilongjiang Academy of Agric Sciences, Harbin, CHINA

Potato late blight (PLB) is the most devastating disease in potato cultivation and the use of fungicides for disease control is widespread. Decision support systems (DSS) are available to help farmers and extension services avoid development of epidemics. Several DSSs are used globally, and knowledge exchange on the development and use of DSSs between the regional PLB stakeholder networks - EuroBlight, AsiaBlight, TizonLatino and USABlight is ongoing. DSSs can include simple tools or more complex platforms, depending on the farmer's socio-economic situation and the rural facilities. A simple hand-held DSS tool that integrates varietal resistance to late blight, precipitation and the time since the last fungicide application was developed by CIP for use in Peru and Ecuador. This tool performed well and is potentially a viable option for small-scale farmers. The current DSSs used in Chile and Argentina have been very successful in improving PLB control, with reductions in fungicides sprays of up to $50 \%$. DSS is a key component of the USABlight approach, with the location and timing of recent late blight observations and near real-time data on the $P$. infestans genotype observed in a specific location being made available. In Europe, the EuroBlight website holds information about 12 different European DSSs and the EuroBlight databases on $P$. infestans genotypes, fungicide efficacy and best management practices aim at supporting the use of the regionally adapted DSSs. In China, a DSS has been developed which predicts when fungicide should be applied and how many applications should be used.

IPM Packages for high value vegetable crops in Cambodia

K. H. SENG, International Development Enterprises iDE- Cambodia, Phnom Penh, CAMBODIA

Pests and diseases are a serious problem for growing vegetables in Cambodia. Many smallholder farmers spray pesticides indiscriminately, harming their health, the environment, and creating pesticide resistance. The IPM Innovation Lab and iDE Cambodia are using IPM technologies to fight pests holistically. The project's focus is on widespread adoption and impact of ecologically-based IPM technologies, practices, and systems. the objective of this project in Cambodia is to decreases crop loss through the adoption of IPM components such as biological and environmental monitoring, predictive monitoring, biological control, insect mating disruption, host plant resistance, grafting, bio-rational pesticides, soil amendments, and habitat management. Examples include the use of Trichoderma, a beneficial fungus that shows significant improvement in crop production for yard long bean, cucumber, Chinese kale and tomato. The project also conducts workshops on plant virus diagnostics to build an infrastructure for Cambodian farmers to receive expert assistance with disease and pest problems.

Quantifying the value of a diagnostic test for early detection surveillance

A. MASTIN (1), F. van den Berg (2), F. Van den Bosch (3), T. R. Gottwald (4), S. R. Parnell (1), (1) University of Salford, Salford, UNITED KINGDOM; (2) Fera Science, Sand Hutton, York, UNITED KINGDOM; (3) Rothamsted Research, Harpenden, ENGLAND; (4) USDA-ARS, Ft Pierce, FL, USA

Advances in molecular diagnostics has resulted in the development of rapid diagnostic tests which can be applied in the field with minimal advance training and can give a result in a short period of time. These "in-field diagnostics" (IFDs) can offer a more sensitive method of pathogen detection during the presymptomatic stage of infection, and have been considered valuable for early detection surveillance. However, the costs of development and application of these tests will be greater than those of visual inspection alone, which may affect the sustainability of an early detection surveillance strategy based upon IFD use. In order to identify whether IFDs are a cost effective alternative to visual inspection (for detection at any specified mean incidence), we have developed a simple heuristic which can be used to compare the total cost of early detection surveillance when using either IFDs or visual inspection. Our method explicitly accounts for the "diagnostic lag" period and the diagnostic sensitivity of the test as well as the rate of pathogen spread in the pathosystem in question. We have applied this heuristic to the case of Phytophthora ramorum in the UK. As well as evaluating the performance of two commercially available IFDs for early detection surveillance, we have investigated the optimal surveillance strategy when the performance of the two detection methods is varied - allowing identification of situations where visual inspection may be optimal.

The rise of fungal canker and vascular diseases in cultivated and native woody plants: A California case study F. TROUILLAS (1), T. J. Michailides (2), A. Eskalen (3), J. R. Urbez-Torres (4), (1) Department of Plant Pathology, UC Davis, Parlier, CA, USA; (2) University of California - Davis, Parlier, CA, USA; (3) Department of Plant Pathology and Microbiology, UC Riverside, Riverside, CA, USA; (4) Agriculture and Agri-Food Canada - Summerland Research and Development Centre, Summerland, BC, CANADA

California leads the world in the production of many fruit and nut crops. Fungal canker and vascular diseases that affect these crops have long been known in California, however, their proliferation in recent years has been of increasing concern. Most California vineyards and orchards are likely to become affected by at least one or more canker or vascular diseases, impacting their yield, longevity and profitability. Canker and vascular diseases are caused by taxonomically unrelated fungal pathogens belonging mainly to the families Botryosphaeriaceae, Diatrypaceae, Diaporthaceae, Togniniaceae and Valsaceae. These pathogens generally infect vines and trees through wounds including pruning wounds, cracks and other injuries and may act alone or jointly to cause disease, sometimes referred as a disease complex. Landscape and native trees in California can also be impacted by similar canker diseases. Alternatively, canker causing fungi may occur as saprophytes or endophytes (latent pathogens) on the native vegetation, which may serve as natural inoculum reservoir for canker diseases affecting fruit and nut crops. In recent years, California has been severely affected by climate change, resulting in long periods of drought. Increased plant stress due to water limitation and heat, increased acreage of fruit and nut crops and the general intensification of agricultural practices are factors that may have contributed to the expansion of fungal canker diseases.

Automated detection of ' $\mathrm{Ca}$. Liberibacter asiaticus' infection in citrus using immune tissue prints and machine learning J. SHAO (1), F. Ding (2), S. Fu (3), J. S. Hartung (4), (1) USDA ARS Molecular Plant Pathology Lab, Beltsville, MD, USA; (2) Huazhong Agricultural University, Wuhan, CHINA; (3) Southwest University, Chongqing, CHINA; (4) USDA ARS MPPL, Beltsville, MD, USA 
Huanglongbing, associated with infection by ' $\mathrm{Ca}$. Liberibacter asiaticus', has caused catastrophic losses to the Florida citrus industry, and is widespread in urban areas of southern California. Infection of trees is followed by erratic distribution of the pathogen and a years-long latent period before symptoms appear but during which time the pathogen can be spread by the psyllid vector. qPCR is used to confirm visual symptoms before regulatory actions are taken, and thus the development of early detection methods is crucial. Immune tissue printing with a rabbit polyclonal antibody that recognizes epitopes of the major outer membrane protein of ' $\mathrm{Ca}$. Liberibacter asiaticus' has been developed. The assay can be scaled to process large numbers of samples. However, scoring the tissue prints as positive or negative for the pathogen requires an expert to view images of the tissue print to determine if individual phloem cells are stained indicating infection. We have begun the development of an automated system using the Tensorflow software to create small Convoluted Neural Networks for image recognition and scoring of the tissue prints. Preliminary results are promising. We have trained our system using known positive and known negative samples obtained from graft-inoculated and qPCR-verified trees. Our results will enable rapid, accurate and unbiased scoring of images for the presence of the pathogen and may facilitate the early removal of infected trees.

Biological control of Striga witch weed in Kenya: From a toothpick to home-grown biocontrol inoculum D. SANDS (1), H. S. Nzioki (2), F. Oyosi (3), C. Baker (4), (1) Montana State University, Bozeman, MT, USA; (2) Kenya Agric Res Inst, Machakos, KENYA; (3) Liberty Initiator Network, Maseno, KENYA; (4) Biotech Investments, Bozeman, MT, USA

Biological control of parasitic plants and weeds in general can be a cost-effective alternative to herbicide use in the hands of smallholder farmers. In the case of control of Striga hermonthica, a serious yield limiting parasitic weed in cereals in sub Saharan Africa, it was necessary to develop selection strategies to produce highly virulent fungal strains for control of this parasitic plant on the maize host. In addition, the fungi had to be made available and affordable to smallholder farmers in a form where they could easily produce fresh inoculum at planting. Three selected biocontrol strains of Fusarium oxysporum fsp. strigae were embedded on toothpicks and sealed in sterile drinking straws, ready to serve as an on-farm primary inoculum. These toothpicks were delivered to 500 smallholder farmers in western Kenya by a network of women trainers who incubated them in boiled rice substrate three days prior to planting. Grains of the rice inoculum were then planted with maize seeds. The resultant maize yields due to the fungi (where paired plots were both fertilized and seeded with hybrid maize) were increased by an average of $56 \%$ in the long rain season and $42 \%$ in the short rain season $(\mathrm{p}<0.0001$, pair-wise $\mathrm{t}$-test). Biocontrol of weeds can be inexpensive and effective if fungal strain selection and appropriate delivery systems are employed, as demonstrated in Kenya.

\section{Liposome delivery system of antimicrobial peptides against Huanglongbing}

J. VELÁSQUEZ GUZMÁN (1), S. Basu (1), R. Rabara (1), L. Huynh (1), G. Basu (1), H. Nguyen (1), J. Shaw (2), Q. Shi (2), S. Zhang (1), E. Stover (2), G. Gupta (1), (1) New Mexico Consortium, Los Alamos, NM, USA; (2) U.S. Horticultural Research Laboratory, USDA-ARS, Fort Pierce, FL, USA

Huanglongbing (HLB), caused by Candidatus Liberibacter spp, is the most devastating citrus disease and threatens citrus production worldwide. Current HLB control strategies are sorely inadequate to prevent spread of the disease. Although both streptomycin and oxytertracycline have been used in foliar sprays, each compound only moderately reduces Liberibacter load, and antibiotic resistant pathogens remain a concern. Here, membrane-targeting, positively-charged amphipathic helical antimicrobial peptides were designed and tested against several bacterial strains in vitro and against Liberibacter in vivo. Peptide P11 exhibit higher antimicrobial activity against culturable bacterial strains, and also exhibit higher activity than streptomycin against Liberibacter. Based on our study of the resistance mechanism of Escherichia coli against P11, we designed a $2^{\text {nd }}$ generation helical amphipathic peptide, $\mathrm{P} 26$, in which two P11 peptides are connected by a 4-amino acid beta turn. P26 is not only more active against $E$. coli, but it is also not susceptible to bacterial resistance. Importantly, P26 has higher anti-Liberibacter activity than either P11 or streptomycin. To effectively deliver these peptides into citrus phloem, we developed virus-like liposome vesicles that encapsulate P11 and P26, using an outer shell of Citrus tristeza virus (CTV) capsid protein. Attachment of virus coat protein to liposome vesicles should ensure transport into and within the plant cells, as well as a controlled release of peptides. We are now testing the efficacy of P11 and P26 by in planta assays using this delivery system. Successful completion of these studies should enable using P11 and P26 for HLB treatment.

Transcriptomic analysis of Ambrosia trifida response to glyphosate: Overlap of cell death pathways between herbicide resistance and pathogen defense? C. VAN HORN (1), T. Gaines (2), K. Ravet (2), E. Patterson (2), R. Beffa (3), S. Gille (3), P. Westra (2), (1) USDA-ARS-PWA, Parlier, CA, USA; (2) Colorado State University - BSPM, Fort Collins, CO, USA; (3) Bayer CropScience, Hochst, GERMANY

Giant ragweed (Ambrosia trifida) is an important weed in US corn and soybean production. The evolution of herbicide resistance and particularly the unique rapid response to glyphosate in this species, not only makes it difficult to manage in cropping systems, but is a highly interesting case of a potential plant response to abiotic stress by utilization of known biotic stress response pathways. The giant ragweed rapid response to glyphosate resembles a programmed cell death response to pathogens seen in other plant species. Due to a lack of genomic resources for giant ragweed, we sequenced the transcriptome of a glyphosate-resistant rapid response individual using Illumina MiSeq and conducted an RNA-seq time course study on three glyphosate-resistant and -susceptible individuals each, before and after glyphosate treatment using Illumina HiSeq technology. De novo assembly using Newbler returned 158,314 contigs, of which 65,623 contigs were greater than or equal to $500 \mathrm{bp}$. From a subset of 143 unique differentially expressed contigs related to plant response to biotic and abiotic stresses, we identified 15 potential candidates involved in the resistance and/or rapid response to glyphosate that could be used for further functional genomics research. This is the first coupled transcriptome sequence and RNA-sequencing experiment of giant ragweed. This transcriptome data may serve as a reference for further gene expression and functional genomics studies, and will provide a valuable molecular resource for future research of herbicide resistance mechanisms in this species as well as other weeds.

Investigating the biology of plant tissue invasion by the rice blast fungus Magnaporthe oryzae N. TALBOT, University of Exeter, Exeter, UNITED KINGDOM Univ of Exeter, Exeter, UNITED KINGDOM

During plant infection, the rice blast fungus Magnaporthe oryzae (syn. Pyricularia oryzae) is able to gain entry to rice leaves using a special infection structure called an appressorium. The infection cell generates enormous turgor, which is focused as mechanical force to breach the rice cuticle. We are studying how appressoria form on the rice leaf surface and how these infection cells function. Appressorium morphogenesis is tightly linked to cell cycle regulation and a specific S-phase checkpoint governs development, while a second S-phase checkpoint is necessary for appressorium function. Penetration peg formation involves re-organisation of the actin cytoskeleton mediated by septin GTPases, controlled by NADPH oxidase activity and a regulated burst of reactive oxygen species in the appressorium. The process is governed by a turgor-sensing mechanism that induces re-polarisation to occur. This involves a novel turgor-sensing kinase that is able to modulate melanisation and solute accumulation, and positively regulate septindependent cytoskeletal re-modellnig and re-polarisation of the appressorium. Following entry into the rice epidermis, M. oryzae is able to locate pit fields and use these site to facilitate its movement between rice cells. We have have found evidence that the Pmk1 MAP kinase cascade is involved in cell-to- 
cell movement by the rice blast fungus. Using a chemical genetic approach, we selectively inhibited the Pmk1 protein kinase, which prevented $M$. oryzae from moving between rice cells. We have investigated how the MAP kinase is able to exert this effect and how it regulates both the suppression of plasmodesmatal-mediated immune responses and septin-dependent hyphal constriction to allow traversal of the rice cell wall during tissue invasion.

Sensitivity of the apple scab pathogen, Venturia inaequalis, to SDHI fungicides

K. AYER (1), M. W. Choi (1), S. M. Villani (2), K. D. Cox (1), (1) Cornell University, Geneva, NY, USA; (2) North Carolina State University, Mills River, NC, USA

Apple scab, caused by the ascomycete Venturia inaequalis, is one of the most economically devastating diseases of apple requiring multiple applications of fungicides throughout the growing season to achieve control. Newer chemistries in the class of succinate dehydrogenase inhibitors (SDHIs) have been registered for use in apple and will become increasingly important as resistance to other fungicide classes becomes more prevalent. To establish protocols for monitoring field resistance, we determined the baseline sensitivity of $V$. inaequalis to all SDHIs registered for use on apple. Furthermore, we analyzed cross-sensitivities of conidial and mycelial growth between six SDHI fungicides for isolates representing both baseline and exposed orchard populations. All SDHI fungicides greatly inhibited conidial and mycelial growth of baseline $V$. inaequalis isolates, with mean $\mathrm{EC}_{50}$ values of conidial growth for all SDHI fungicides ranging from 0.0016 to $0.176 \mu \mathrm{g} \mathrm{ml}^{-1}$. Preliminary trends indicate some correlation in sensitivity between all SDHI fungicides. Isolates with previous exposure to fungicides show a decreased level of sensitivity to SDHI fungicides when compared to baseline isolates, suggesting quantitative resistance mechanism may be playing a role in isolate response. Thus far, no mutations in the target $s d h$ target genes have been identified. Phenotypic and genotypic assessment is ongoing to continually monitor resistance development.

Stability of the Spread Parameter of the Power Law Model for Dispersal Gradients of Disease Epidemics

P. S. OJIAMBO (1), G. David (2), L. Mehra (3), D. Christie (1), R. D. Magarey (1), (1) North Carolina State University, Raleigh, NC, USA; (2) USDA ARS, Corvallis, OR, USA; (3) US Horticultural Research Lab, Fort Pierce, FL, USA

Theory and experimental evidence indicate that pathogens that are aerially transmitted over long distances typically have a marginal contact distribution that has a power law tail. Epidemics caused by these pathogens are characterized by a dispersive wave with accelerating velocity of focus expansion. In several plant and animal disease systems, the spread parameter $(b)$ of the power law model has been shown to be approximately 2 . This approximation facilitates prediction of the distance travelled by epidemic fronts of dispersive waves. Several factors influence disease spread and expansion of the resultant epidemic, although the stability of $b$ over multiple realizations of an epidemic has not been established. Using cucurbit downy mildew in the eastern United States as a case study, epidemic data collected from 2008 to 2014 were analyzed using a spatio-temporal model of disease spread that incorporates logistic growth in time with a power law function for dispersal. Gradients from temporal and spatial regression models varied by a factor of 2.5 across years, with the corresponding estimates of $b$ ranging from 1.51 to 4.16 . Covariance analysis showed a significant interaction between $b$ and time even where data were well described by the power law model. These results indicate that $b$ may not be stable over multiple epidemic years and a value of 2 may be considered the lower limit of the parameter for organisms capable of long-distance dispersal when epidemics are not severely constrained by factors such as host availability.

Monitoring the spread of Maize chlorotic mottle virus and Sugarcane mosaic virus under high disease pressure in Ecuador E. Cañarte-Bermudez (1), J. Navarrete (1), R. Solorzano (1), A. Mendoza (1), J. F. Cornejo (2), R. A. Alvarez-Quinto (3), B. E. Lockhart (4), D. QUITOAVILA (5), (1) Instituto Nacional de Investigaciones Agropecuarias, Estacion Portoviejo INIAP, Portoviejo, ECUADOR; (2) Escuela Superior Politecnica del Litoral, Guayaquil, ECUADOR; (3) Centro de Investigaciones Biotecnologicas del Ecuador, Guayaquil, ECUADOR; (4) Department of Plant Pathology, University of Minnesota, St Paul, MN, USA; (5) Escuela Superior Politecnica del Litoral, Facultad Ciencias de la Vida, Guayaquil, ECUADOR

Maize lethal necrosis (MLN) is currently the most devastating viral disease in corn. The disease is caused by a mixed infection involving Maize chlorotic mottle virus (MCMV) and Sugarcane mosaic virus (SCMV). Although identified as early as 1970's, MLN has re-emerged during the past 6 years as the major problem in several countries around the world. In Ecuador, the disease was documented in 2015 in yellow corn cultivars, mostly grown in coastal provinces, where the presence of both MCMV and SCMV was confirmed in severely affected plants. However, epidemiology aspects regarding the time of the year when MCMV and SCMV appear in the field, as well as the rate of spread and their prevalence in relationship to insect populations remained unknown. To shed light on the dynamics of MLN in Ecuador, a field experiment was implemented where maize plots were set up in a contiguous overlapping fashion. Plots were planted in 30-day intervals starting from January. In each plot, 25 plants were marked for monitoring the presence of MCMV and SCMV in relationship with insect populations (aphids, thrips and leaf beetles) that were recorded in a weekly basis during one year. Our results indicate at least two peaks of virus infection along the year, with SCMV showing the highest rate of spread compared to MCMV. Aphids (Rhopalosiphum maidis) and thrips (Frankliniella spp) were the most prevalent insects exhibiting population peaks correlated to virus incidence.

Marrying classical with inundative weed biological control

M. SEIER, D. Kurose, H. C. Evans, CABI, Egham, Surrey, UNITED KINGDOM

Traditionally, classical and inundative weed biocontrol have been considered as two separate approaches. The former targets invasive alien weeds by releasing exotic, co-evolved and host-specific natural enemies from the weed's native range to achieve self-perpetuating control; whilst the latter aims to increase the efficacy of a native agent by repeated mass application of high concentrations of infective propagules to create an epiphytotic. However, this distinction can become blurred when a classical agent is applied inundatively against an invasive weed, such as the rust Maravalia cryptostegiae against rubbervine (Cryptostegia grandiflora) in Australia, or when a pathogen strain from one country is licensed as a bioherbicide in another. The leaf-spot pathogen Mycosphaerella polygoni-cuspidati on Japanese knotweed, however, represents a unique example of marrying the classical and inundative approaches. This Japanese fungus had been considered as a classical agent for the weed in both the UK and Canada, but was disregarded due to potential impacts on native plant species. However, M. polygoni-cuspidati is heterothallic, lacking an asexual morph and able to infect via mycelial fragments. This offers the opportunity for mass production and targeted single-mating type inoculum application, whilst preventing reproduction, persistence and spread of the agent in the environment.

Role of quarantine in exclusion of transboundary plant viruses: A developing country's perspective

C. C. VASIMALLA, D. R. B. Parakh, P. Kumari, A. K. Maurya, S. C. Dubey, ICAR-National Bureau of Plant Genetic Resources, New Delhi, INDIA

The global movement of agricultural produce has the potential to introduce new pests including viruses endangering the agriculture of importing country Viruses are one of the important yield-reducing factors in crops. The imported germplasm including transgenics is subjected to quarantine processing at 
ICAR-National Bureau of Plant Genetic Resources, New Delhi. The strategies for exclusion of viruses in quarantine include post-entry quarantine growing/inspection, electron microscopy, ELISA and RT-PCR. Forty five viruses were intercepted during the past two decades including 19 viruses: Barley stripe mosaic virus, Bean mild mosaic virus, Bean pod mottle virus, Broad bean mottle virus, Broad bean stain virus, Broad bean true mosaic virus, Cherry leaf roll virus, Cowpea mottle virus, Cowpea severe mosaic virus, Dioscorea latent virus, Garlic virus C, High plains virus, Maize chlorotic mottle virus, Pea enation mosaic virus, Peanut sunt virus, Pepino mosaic virus, Raspberry ringspot virus, Tomato ringspot virus and Wheat streak mosaic virus not reported and 21 viruses not known to occur on particular host(s) in India. The introduction of 19 exotic viruses was averted. Availability of antisera, viral genome sequences, detecting an unknown/ exotic virus etc., are the key challenges in virus detection in quarantine. The right strategy and appropriate technique for virus detection are essential to ensure biosecurity of crops from transboundary plant viruses.

\section{Iron tissue content suppresses Cercospora leaf blight development in soybean}

E. SILVA (1,2,3), A. K. Chanda (4), T. G. Garcia (5), C. L. Robertson (6), E. Tubana (7), R. W. Schneider (5), (1) LSU, Baton Rouge, LA, USA; (2) Valent U.S.A. LLC, Seymour, IL, USA; (3) Louisiana State Univ, Agricultural Center, Baton Rouge, LA, USA; (4) Department of Plant Pathology, University of Minnesota, Crookston, MN, USA; (5) Louisiana State University, Baton Rouge, LA, USA; (6) Louisiana State University Agricultural Center, Baton Rouge, LA, USA; (7) Louisiana State University Agricultural Center Plant Pathology Department, Baton Rouge, LA, USA

Cercospora leaf blight of soybean (CLB), caused by Cercospora kikuchii, C. cf. flagellaris and C. cf. sigesbeckia, is a devastating disease in Louisiana and many other southern U.S. states. C. cf. flagellaris is the main Cercospora species associated with CLB in the U.S. A preliminary micronutrient screening of Al, B, Cu, Fe, Mn, Mo, and, Zn showed that foliar applications of Fe consistently decreased CLB severity. The objective of this study was to determine if $\mathrm{Fe}$ foliar applications (Manny Plex Fe and Fe EDTA, Brandt Consolidated, Springfield, IL) would reduce soybean leaf colonization by $C$. cf. flagellaris and the severity of CLB leaf symptoms (purple and blight). The cultivar Pioneer 95Y61 was treated with four rates of Manny Plex Fe and Fe EDTA under field conditions at R5 growth stage. Leaf tissue analyses and qPCR were performed to verify Fe uptake and fungal leaf colonization, respectively. Results showed a low relationship between leaf colonization by the pathogen and the leaf symptoms assessed (purple and blight). Severity of purple leaf symptoms increased as leaf Fe concentration increased up to $230 \mathrm{mg} / \mathrm{kg}$ of dry matter, after this threshold, the purple symptom severity tended to decrease. Blight symptoms were suppressed as the Fe concentration in soybean leaves increased up to $350 \mathrm{mg} / \mathrm{kg}$. Moreover, leaf Fe concentration did not affect the biomass of the fungus. There was a positive relationship between leaf concentrations of Fe and yield. Negative relationships between yield and Cercospora biomass and severity of blight and purple symptoms were observed. Taken together, the results suggested that Fe does not affect pathogen growth, but may influence its virulence. Furthermore, the results showed that disease suppression is only achieved when the levels of $\mathrm{Fe}$ is well above the optimal physiological range $(>100 \mathrm{mg} / \mathrm{kg})$ for soybean growth and development.

Endophytic microrganisms for silverleaf disease (Chondrostereum purpureum) control in apple D. GRINBERGS (1,2), N. Padilla (1), Y. Robles (1), E. A. Moya-Elizondo (2), A. France (3), (1) Instituto de Investigaciones Agropecuarias, Chillán, CHILE; (2) Universidad de Concepción, Chillán, CHILE; (3) INIA Quilamapu, Chillan, CHILE

Silverleaf is an important disease which produces foliar silvering, wood necrosis and reduction of fruit yield and quality. Field studies have shown spontaneous reversion of foliar symptoms, together with yield recovery. Plants are clones, thus it is postulated that resistance is driven by a non-genetic mechanism, like their microbiome. The objectives were to explore plants endophytic microbiome, to determine endophytes antagonistic activity against the pathogen, and their ability to colonize apple plants. Microorganisms were isolated from the internal trunk of diseased and reverted plants and confronted to four C. purpureum strains. Isolates showing the greatest antagonism were selected for further tests using their secondary metabolites and inoculating in vitro apple plants by drenching. Microbial diversity was greater in reverted plants (73\%): $48 \%$ actinobacteria, $30 \%$ fungi and $13 \%$ bacteria. Isolates from diseased plants were not antagonistic, while $37 \%$ of the reverted ones showed activity. They were identified and morphologically, biochemically and genetically characterized. Streptomyces spp. isolates inhibited C. purpureum growth by 66 to $62 \%$, without differences among bacterial plugs and wells containing metabolites $(\mathrm{P}<0.05 \%)$. In addition, C. purpureum growth was inhibited in $85-96 \%$ when metabolites were spread on the agar. Moreover, Streptomyces strains successfully colonized apple plants as endophytes, becoming a promising alternative for silverleaf control.

\section{Fusarium tropical race 4 a disease threating a global smallholder and industrial crop} M. A. DITA RODRIGUEZ (1), C. Staver (2), (1) Embrapa, Jaguariúna, SP, BRAZIL; (2) Bioversity International, Montpellier, FRANCE

Bananas mean food security and livelihoods for over 400 million people worldwide. Despite of the importance of bananas as a global commodity, about $85 \%$ are consumed in their country of origin. Pest and diseases are major challenges for banana diversity and productivity, especially Fusarium wilt (FW), caused by Fusarium oxysporum f. sp. cubense (Foc). In the 1950s, Foc race 1 disrupted the banana industry forcing the replacement of the susceptible cultivar Gros Michel with the resistant Cavendish. Cavendish plantations, currently about $\mathrm{xx} \%$ of total global banana production, remained unaffected until, a new strain, tropical race 4 (TR4), was identified in the 1980s in Asia. Since that time, TR4 has destroyed over 100.000 ha of Cavendish in Asia and continues to spread to new countries in Asia and into the Middle East and Africa. In spite of being decreed a quarantine disease throughout Latin America and the Caribbean (LAC), TR4 risk analyses suggests the disease will eventually reach major banana plantations. Banana producing countries in LAC are especially concerned as no containment measures are reported in place in any TR4-affected country, excepting Australia TR4 not only affects Cavendish, but many local banana cultivars important for sale and food security. Testing the susceptibility of banana diversity to TR4 is still ongoing, but preliminary results suggest that many local cultivars are threatened. Therefore, exclusion and containment are top priorities anywhere where TR4 is absent. Traveler alerts and contingency plans, implemented in only a few countries in LAC, are extremely necessary. In this work we present comprehensive data about the global threat posed by TR4 giving directions towards exclusion, preparedness and research priorities.

\section{What's in a name? Emergent strains, admixtures and fuzzy species in Ceratocystis} T. HARRINGTON, Iowa State University, Ames, IA, USA

Phylogenetic analyses may identify lineages, but are they strains or species? Strains of Ceratocystis are readily dispersed by humans, and Ceratocystis wilt is a major emerging disease on fruit and forest trees in plantations, cities, and native ecosystems around the world. In the last 25 years, the species $C$. fimbriata has been elevated to its own genus and split into 36 species, mostly circumscribed by phylogenetic analyses alone, without diagnostic phenotypic characters or comparisons to natural populations. Most South American isolates and some of the new species form a monophyletic group that is sexually compatible with the original $C$. fimbriata, a sweet potato strain that may have originated in Ecuador. This biological species includes five recently named species, which appear to be strains. At least two of these strains were introduced to Asia and are fully interfertile in controlled crosses. Admixtures of the two are evident in Asia, where major losses are seen on pomegranate, mango, Acacia, and Eucalyptus. Purely molecular species concepts may inappropriately designate strains as species and suggest that they are genetically isolated. However, application of the biological species concept suggests that introduced strains of $C$. fimbriata have the potential to recombine. A good species name conveys a wealth of information and may lead to a better understanding of the epidemiology and management of new outbreaks, as well as better quarantine restrictions. 
Structure-guided protein engineering extends immune receptor recognition of effectors from the rice blast fungus

J. C. DE LA CONCEPCION (1), M. Franceschetti (1), H. Saitoh (2), R. Terauchi (3,4), S. Kamoun (5), M. Banfield (1), (1) John Innes Centre, Norwich, UNITED KINGDOM; (2) Tokyo University of Agriculture, Tokyo, JAPAN; (3) Kyoto University, Kyoto, JAPAN; (4) Iwate Biotechnology Research Center, Iwate, JAPAN; (5) The Sainsbury Laboratory, Norwich, UNITED KINGDOM

Pathogens deliver an array of molecules, termed effectors, to manipulate host cellular processes for their own benefit. Perception of effectors by intracellular immune receptors from the NLR (Nucleotide-binding, Leucine-rich Repeat) family triggers immune responses, leading to cell death and preventing the spread of the pathogen. The engineering of new NLR receptors with improved recognition capabilities has been a long-term goal in plant biotechnology. However, it remains elusive due to the narrow recognition spectrum of NLRs, and the lack of mechanistic knowledge about immune activation. The rice NLR Pikp recognizes the rice blast pathogen effector AVR-PikD by direct binding to an integrated Heavy Metal Associated (HMA) domain, resulting in disease resistance. However, polymorphic effector variants have lower binding affinity, and evade immune recognition. Here, we exploit this knowledge to investigate the mechanistic basis of a natural allele of rice Pik, Pikm, with broader recognition specificities for effector variants. Biochemical characterization of Pikm/AVR-Pik interactions revealed a causal link between higher binding affinities in vitro and immune response in planta. The crystal structures of five different receptor/effector combinations uncovered the key amino acids that underpin differential binding specificities. We applied this information to generate mutations in the rice NLR Pikp that increased binding affinity for the effector variants. These mutants acquired gain of immune response to previously unrecognized effectors. Therefore, we have successfully engineered new immune receptors with the potential to improve disease resistance in crops.

After the data deluge: Biological characterization of the new variants and viral species identified by NGS

S. MASSART (1), T. Candresse (2), J. Gil (3), C. Lacomme (4), L. Predajna (5), M. Ravnikar (6), J. S. Reynard (7), A. Rumbou (8), P. Saldarelli (9), D. Škorić (10), E. Vainio (11), J. Valkonen (12), H. Vanderschuren (13), T. Wetzel (14), (1) University of Liège - Gembloux Agro-Bio Tech, Gembloux, BELGIUM; (2) INRA Bordeaux, UMR 1332 BFP, Villenave d'Ornon, FRANCE; (3) Swedish University of Agricultural Sciences SLU, Uppsala, SWEDEN; (4) Science and Advice for Scottish Agriculture (SASA), Edinburgh, UNITED KINGDOM; (5) Biomedical Research Center SAS, Institute of Virology, Bratislava, SLOVAKIA; (6) National Institute of Biology, Ljubljana, SLOVENIA; (7) Agroscope, Nyon, SWITZERLAND; (8) Humboldt University Berlin, Berlin, GERMANY; (9) CNR Institute for Sustainable Plant Protection, Bari, ITALY; (10) University of Zagreb, Faculty of Science, Department of Biology, Zagreb, CROATIA; (11) Natural Resources Institute Finland, Helsinki, FINLAND; (12) University of Helsinki, Helsinki, FINLAND; (13) University of Liège Gembloux Agro Bio-Tech, Gembloux, BELGIUM; (14) DLR Rheinpfalz, Neustadt, GERMANY

Recent advances in high-throughput sequencing technologies and bioinformatics have generated huge new opportunities for discovering and diagnosing plant viruses and viroids. Plant virology has undoubtedly benefited from these new methodologies, but at the same time, faces now substantial bottlenecks, namely the biological characterization of the newly discovered viruses and the analysis of their impact at the biosecurity, commercial, regulatory, and scientific levels. This paper proposes a scaled and progressive scientific framework for efficient biological characterization and risk assessment when a previously known or a new plant virus is detected by next generation sequencing (NGS) technologies.

Synaptic guidepost protein (syg-2) gene-specific primers for detecting Bursaphelenchus xylophilus X. WANG, L. Wang, Research Institute of Forest Ecology, Environment and Protection, CAF, Beijing, CHINA

Pine wilt disease (PWD) caused by pine wood nematode (PWN) is a worldwide quarantine disease that is detrimental to pine trees. A stable and highly specific Bursaphelenchus xylophilus gene helps to accurately diagnose diseased pine trees. This study standardizes the PCR and loop-mediated isothermal amplification (LAMP) methods using newly designed primer sequences of the syg-2 gene, which encodes the synaptogenesis protein syg-2, was involved in many major physiological functions and was conserved in B. xylophilus, the region showed 46\% dissimilarity (i.e., 211 in $398 \mathrm{bp}$ is identical) between B. xylophilus and B. mucronatus, while this dissimilarity in Nuclear ribosomal internal transcribed spacer (ITS) region was $20 \%$. Our experimental results suggest that the syg-2 gene makes a good distinction between B. xylophilus and other nematodes. However, when using the ITS region design primers for LAMP detection, $B$. frandulentus and $B$. doui could be detected in about 50 minutes, and B. mucronatus began to be detected in about 90 minutes. Therefore, syg-2 gene can be used as a new target gene for detection of PWN.

Phage therapy to manage bacterial canker in kiwifruit caused by Pseudomonas syringae pv. actinidiae C. S. OH, Department of Horticultural Biotechnology, Kyung Hee University, Yongin, KOREA

Bacterial canker in kiwifruit trees caused by Pseudomonas syringae pv. actinidiae $(P s a)$ is very problematic worldwide. Due to the limitation of usage of agricultural antibiotics or copper compounds for disease management, alternative methods need to be developed. Recently, bacteriophages, viruses that specifically kill target bacteria, have been reconsidered as potential biological control agents for bacterial pathogens due to their specificity and safety to environment. In this study, 77 bacteriophages effective to Psa strains, including the Psa2 and Psa3 groups, were isolated from soils collected from kiwifruit orchards in South Korea, and seven bacteriophages were selected based on restriction enzyme digestion patterns of genomic DNA and their host range. Two belonged to the Myoviridae family and five belonged to the Podoviridae family, based on their morphology observed with transmission electron microscope. They were effective to not only Psa strains, but also other diverse P. syringae pathovars. Lytic activity of the selected bacteriophages was sustained in vitro until 80 hours, and their activity remained stable up to $50^{\circ} \mathrm{C}, \mathrm{pH} 11$, and under UV-B light. To examine their control efficacy against $P s a$, bacteriophage cocktails mixed with three or five bacteriophages were sprayed to one-month-grown grafted kiwifruit trees in the greenhouse 3 hours before spraying with $P s a$. Treatment of bacteriophage cocktails with $10^{8}$ plaque forming units $/ \mathrm{ml}$ could protect kiwifruit trees from $P s a$ up to almost $90 \%$ of Agrimycin ${ }^{\circledR}$ control efficacy. These results indicate that selected bacteriophages could be useful for phage therapy against $P s a$ in kiwifruit trees and also probably other P. syringae pathovars.

Molecular mechanism of reactive oxygen species in regulating the development and pathogenicity of Botrytis cinerea S. TIAN, Z. Zhang, B. Li, G. Qin, T. Chen, Institute of Botany, Chinese Academy of Sciences, Beijing, CHINA

Reactive oxygen species (ROS) play crucial role in the interaction between plants and pathogens. NADPH oxidase complex (Nox) is the major enzymatic producer of ROS in eukaryotic cell. However, the underlying molecular mechanism of Nox remains largely unknown in Botrytis cinerea, a notorious necrotrophic plant pathogen. We found that the knockout mutant of BcNoxR, the regulatory subunit of Nox, exhibited reduced growth, sporulation, virulence, and altered ROS distribution. The protein abundance of 6-phosphogluconate dehydrogenase (BcPGD) and actin (BcActin) could be influenced by BcNoxR. Genetic analysis indicated that BcPGD and BcActin were critical pathogenic determinants. Moreover, the polymerization of actin cytoskeleton was disturbed in $\Delta b c n o x R$. We also proved that BcNoxR could regulate the secretion of extracellular proteins, including some 
pathogenic factors. Analysis of redox proteomics revealed that BcNoxR was essential for maintaining the equilibrium of redox status in $B$. cinerea. Cys thiols in $\triangle b c n o x R$ mutant were more oxidized than those in wild-type. In addition, we further confirmed that aquaporin AQP8 is a key regulator in the ROS transport and distribution. Deletion of $A Q P 8$ completely inhibited the development infection structures and disturbed polarity distribution of ROS in $B$. cinerea. These data lead to a better understanding of the ROS production, transport, and regulatory roles in development and pathogenicity of necrotrophic pathogens.

\section{Crystals to crops: Using host targets of a rice blast pathogen effector protein to engineer a plant immune receptor with novel recognition} specificity

J. MAIDMENT (1), M. Franceschetti (1), C. Jantasuriyarat (2), H. Saitoh (3), A. Maqbool (4), R. Terauchi (5), S. Kamoun (4), M. Banfield (1), (1) John Innes Centre, Norwich, UNITED KINGDOM; (2) Kasetsart University, Bangkok, THAILAND; (3) Tokyo University of Agriculture, Tokyo, JAPAN; (4) The Sainsbury Laboratory, Norwich, UNITED KINGDOM; (5) Iwate Biotechnology Research Center, Iwate, JAPAN

Magnaporthe oryzae causes rice blast disease and is a major threat to global food security. During infection, this pathogen secretes effector proteins which manipulate host targets to promote virulence. Some effectors are recognised by specific nucleotide-binding leucine rich repeat (NLR) proteins in rice to trigger plant defences. Recognition of the effector AVR-Pik is mediated by the paired rice NLRs Pik-1/Pik-2. An integrated heavy metal associated (HMA) domain was previously identified in Pik-1, to which certain alleles of AVR-Pik directly bind to trigger disease resistance. Multiple alleles of Pik-1 have evolved in rice and display differential responses to different AVR-Pik effectors. However, the stealthy effector alleles AVRPikC/AVR-PikF do not interact with any Pik-1 alleles, evading plant defences. A family of small HMA domain-containing (sHMA) proteins have been identified as the host targets of AVR-Pik. Analytical gel filtration revealed that AVR-PikC/AVR-PikF bind to sHMA1 in vitro. We hypothesised that modifying the HMA domain of Pikp-1 to resemble sHMA1 would extend the response of the NLR to AVR-PikC/AVR-PikF. To guide engineering, we solved the crystal structure of AVR-PikF in complex with sHMA1. Using this, we produced a novel NLR which triggered an immune response to AVRPikC in Nicotiana benthamiana. This study reveals a novel approach to engineer improved NLR proteins capable of triggering immunity to previously unrecognised effectors.

Developing durable genetic solutions to crop diseases D. M. HORVATH, 2Blades Foundation, Evanston, IL, USA

Useful solutions to crop diseases can be found in modern plant science, where enormous advances have occurred in the understanding of how diseasecausing microbes bypass or suppress plant defenses. These discoveries have lead directly to new ways to extend or reinforce a plant's natural immunity and produce crops with long-lasting resistance. Yet, there is a poor history of implementation of such discoveries. The 2 Blades Foundation, was founded to address this bottleneck and contribute to global food security by identifying, advancing, and delivering solutions for crop disease. Through development programs addressing significant diseases of wheat, soybean, potato, tomato and other crops, it supports the foremost scientists and advances the most promising discoveries into practical applications to produce sustainable, environmentally-friendly genetic solutions. 2Blades partners with foundations, companies, and international agricultural development organizations that have effective track records for the delivery of these innovations into the hands of growers.

In vitro and in vivo evaluation of microbial agents for management of rice blast disease in Tanzania

I. HASHIM (1), D. Mamiro (1), R. Mabagala (1), T. Tefera (2), (1) Sokoine University of Agriculture, Morogoro, TANZANIA; (2) International Center of Insect Physiology \& Ecology (icipe), Addis Ababa, ETHIOPIA

The main objective of this study was to evaluate the effect of two microorganisms, Trichoderma asperellum and Bacillus subtilis, on the management of rice blast disease caused by Pyricularia oryzae. The dual culturing technique and pot experiment was conducted to test the antagonistic effect of $T$. asperellum and $B$. subtilis against $P$. oryzae. Both $T$. asperellum and $B$. subtilis showed a high antagonistic activity with a percentage inhibition radial growth (PIRG) $>75 \%$ compared to Linkimil $72 \mathrm{WP}$ with PIRG $21-23 \%$ and the negative control PIRG $0 \%$. In a pot experiment, disease incidence (DI) and percent disease severity (PDS) 14 days after inoculation (DAI) and 21 DAI indicated that DI increased by $11.34 \%$ on plants treated with B. subtilis, followed by T. asperellum (7.26\%), and Linkimil $72 \mathrm{WP}(0.15 \%)$ compared to the control (no spray) (19.94\%). Similar trends were observed on disease severity, which increased by $8.2 \%$ for $B$. subtilis followed by T. asperellum $(0.30 \%)$ and Linkimil $72 \mathrm{WP}(0.78 \%)$ compared to the negative control $(9.17 \%)$. The increase in lesion size was significantly low $(0.15 \mathrm{~mm})$ in the plants treated with $T$. asperellum, followed by $B$. subtilis $(0.21 \mathrm{~mm})$, and Linkimil $72 \mathrm{WP}(0.47 \mathrm{~mm})$ compared to the negative control $(0.91 \mathrm{~mm})$.

Functional characterization of polyketide synthase genes in the biocontrol fungus Clonostachys rosea U. Fatema (1), A. Broberg (2), D. F. Jensen (1), M. Karlsson (1), M. DUBEY (1), (1) Dept. Forest Mycology and Plant Pathology, Swedish University of Agricultural Sciences, Uppsala, SWEDEN; (2) Dept. of Molecular Sciences, Swedish University of Agricultural Sciences, Uppsala, SWEDEN

Polyketides, a group of secondary metabolites produced both by prokaryotes and eukaryotes, are vital for various biological functions required for ecological and evolutionary adaptation in microorganisms. These metabolites are biosynthesized by large, multi-domain enzymes called polyketide synthases (PKSs). The aim of this study was to investigate the biological functions of PKSs in the biocontrol fungus Clonostachys rosea, with emphasis on their role in biocontrol interactions. The $C$. rosea genome contains 31 PKS genes, significantly higher compared with the number found in the more studied Trichoderma fungal biocontrol agents. Seventy-five percent of the $C$. rosea PKSs are arranged in biosynthetic gene clusters, and gene expression analysis during $C$. rosea interactions with the fungal pathogens Botrytis cinerea and Fusarium graminearum showed common and species-specific induction of PKS genes. There was a positive correlation between PKS gene expression and antagonism in C. rosea. The pks 22 and $p k s 29$ genes were highly induced during fungal-fungal interactions, and gene deletion studies revealed that PKS29 was required for full antagonism against $B$. cinerea, and for biocontrol of fusarium foot rot on barley. Metabolite analysis of $C$. rosea wildtype and deletion strains using LC-MS and NMR revealed that $\Delta p k s 22$ strains lost the ability to produce a previously unknown polyketide with the molecular formula $\mathrm{C}_{14} \mathrm{H}_{25} \mathrm{NO}_{3}$, while $\Delta p k s 29$ strains showed a significant $(\mathrm{P}$ $=0.001) 50 \%$ reduction in production of another unknown polyketide with the molecular formula $\mathrm{C}_{15} \mathrm{H}_{28} \mathrm{O}_{3}$. This study shows the involvement of PKSs in fungal biocontrol, and led to the identification of previously unknown polyketides in C. rosea.

Challenges for late blight control in developing countries A. NJOROGE, International Potato Center, Nairobi, KENYA Swedish University of Agricultural Sciences (SLU), Uppsala, SWEDEN 
Managing late blight (LB) is tough in low-income countries (LIC) due to limited farmer knowledge, variable cropping systems, lack of inputs and technology and weak R\&D bodies. LIC also have susceptible cultivars and a variable pathogen population and tackling these issues needs a multi-faceted tactic plus use of novel skills. To increase host resistance use, new resistant genotypes have been evaluated and improved methods for rating resistance developed. Recently, it's been shown that use of intermediate levels of resistance in developing countries is more widespread than had previously been thought. Complementary to this, genetically improved varieties with $3 R$ genes have been field tested in Uganda through collaborative efforts. To better understand pathogen variability and its effects for control, partners in Europe and LIC have promoted globalization of EuroBlight marker tools and online platforms. Now, researchers in Africa are promoting creation of AfricaBlight to standardize LB research in this region. Using SSR markers in Eastern Africa has revealed KE-1 lineage that is rapidly displacing the dominant US-1 and is expected to cause greater LB management issues. To understand epidemiology of late blight in varied environments, LB modelling capacity has been developed and models parameterized for highland tropical conditions, allowing prediction of climate change effect on LB. Advance of simple DSS to low-resource farmers are vital to improving farmer knowledge.

Timing is everything: Stomatal manipulation facilitates Puccinia graminis entry in dark, resulting in counter evolution of barley Rpg5 immune receptor

S. SOLANKI (1), G. Ameen (2), R. Sharma Poudel (3), P. Borowicz (4), R. S. Brueggeman (2), (1) Plant Pathology Department, North Dakota State University, Fargo, ND, USA; (2) Department of Plant Pathology, North Dakota State University, Fargo, ND, USA; (3) North Dakota State University, Fargo, ND, USA; (4) Animal Sciences, NDSU, Fargo, ND, USA

The integrated decoy hypothesis explains the evolution of dual plant NLR resistance gene loci, where one NLR partner contains an Integrated $\underline{\text { Sensory }}$ Domain (ISD) that represent virulence effector targets translocated to NLRs to function as pathogen baits. The barley NLRs, Rpg 5 and $H v R g a \bar{l}$, are both required for resistance to Puccinia graminis (Pg), the stem rust pathogen. Functional Rpg5 alleles have a protein kinase (PK) ISD, and the putative progenitor of the ISD was identified and designated guard cell associated kinase 1 (Gakl). Comparative analysis shows that Gakl is a paralog of the Arabidopsis stomatal protein kinase required for light responsive stomatal opening. Confocal laser microscopy determined $P g$ entry through stomata in the dark and qPCR on cDNA from laser microdissected stomata showed high levels of Gakl compared to whole leaf samples, supporting the hypothesis that $P g$ may manipulate stomata localized Gak1, allowing for stomata opening and pathogen entry at night, a new paradigm in $P g$-barley interaction. Y2H identified HvVOZ1 as an Rpg5 ISD interactor and in Arabidopsis it has been shown that Atvoz1/Atvoz2 mutants have reduced stomatal opening. AtVoz1 also interacts with the PhyB far red light receptor, thus we hypothesize interplay between Gak1/HvVoz1 and a $P g$ effector that manipulates the stomates to open allowing entry during the night. However, barley counter evolved a Rpg5-Gak1 ISD to recognize Gak1 manipulation resulting in resistance responses.

Cherry canker genetics - applying genomics to the control of perennial disease problems in fruit trees M. HULIN (1), A. D. Armitage (1), K. Housley (1), J. W. Mansfield (2), R. W. Jackson (3), R. Harrison (1), (1) NIAB EMR, East Malling, UNITED KINGDOM; (2) Imperial College London, London, UNITED KINGDOM; (3) University of Reading, Reading, UNITED KINGDOM

Pseudomonas syringae pathovars are highly specialised, with individual strains only infecting one or a few hosts. It is believed this host specificity is due to the repertoire of type III effector proteins involved in both virulence and avirulence in planta. This topic was explored using comparative genomics of three divergent clades that have convergently evolved to cause bacterial canker on cherry (Prunus avium). The clades include $P$. syringae pv. morsprunorum (Psm) races 1 and 2 (which are now known to be distantly related) and P. syringae pv. syringae (Pss). Three reference isolates of Psm R1, R2 and Pss were sequenced with PacBio and the genomes of a diverse set of further strains were sequenced using the Illumina MiSeq. Genomic analysis of the Prunus strains has revealed highly divergent effector and toxin repertoires within and between the different clades, indicating they use distinct mechanisms to cause bacterial canker. A bayesian approach was utilised to predict effectors whose evolution is significantly associated with the evolution of pathogenicity for cherry. Candidate virulence effectors have been gained via horizontal gene transfer which has been both plasmid and phage mediated. By contrast, the HopAB effector family has been lost or truncated in cherry-pathogenic clades. Members of this effector family were cloned and ectopically expressed in pathogenic strains and found to trigger immunity, leading to avirulence on cherry leaves. Work is now underway to identify the role of conserved effectors in virulence. This is in parallel with work on the host looking for both effector-driven and more quantitative host resistance mechanisms.

Diversity and invasion resistance relationships in rhizosphere microbial communities with consequences to soilborne disease suppression G. VADAKATTU (1), S. M. Neate (2), (1) CSIRO, Glen Osmond, AUSTRALIA; (2) University of Southern Queensland, Toowoomba, AUSTRALIA

Rhizosphere microbial communities can play an important role in the defence against plant pathogens and soilborne diseases. The presence of antagonistic microflora, spatial and temporal fluctuations in the soilborne pathogens, their antagonists and other rhizosphere microbes have consequences to disease impacts. In our work on biological suppression, in a targeted polyphasic approach, the functional and compositional attributes of bacterial and fungal communities, specific functional groups, catabolic diversity and interactions were explored in field and bioassay experiments. Suppressive soils generally exhibited greater community metabolic diversity, higher bacterial species richness and genetic diversity compared to that in non-suppressive soils. Exposure of soils to factors that promote disease suppression had greater influence on rhizosphere bacterial composition in suppressive soils. Also, multiple bacterial taxa with varying physiological and metabolic capabilities contributed to differences between suppressive and non-suppressive soils. As bacterial communities can respond rapidly to changes in seasonal conditions, greater diversity allows the plant select different members that contribute to similar functions. Suppressive fungal communities were characterized by higher diversity, higher connectedness, and more nodes indicating resilience to change. In highly connected biological networks, efficient resource exploitation by all species including niche overlap between pathogen and community can occur thus reducing opportunities for pathogen invasion, growth and disease incidence thereby improving temporal stability of disease suppression capacity. High genotypic richness and dissimilarity exert significant influence in determining the susceptibility of ecosystems for pathogen invasion.

Development of Disease Management Options for Pseudocercospora Fruit and Leaf Spot in Teso Region of Uganda J. ADRIKO, National Agricultural Research Laboratories (NARL), Kampala, UGANDA

Pseudocercospora is the most prevalent disease observed on citrus fields in Teso region, Eastern Uganda with average disease incidence reaching over $80 \%$ and mean disease severity scores of up to 3.5. Disease management experiments were set up for pseudocercospora fruit and leaf spot on 24 fields in Ngora and Soroti districts in the Teso region, with 12 experiments run from second season of 2016 (referred hereafter as 2016B) and the other 12 from the first season of 2017 (2017A). In 2016B, 6 treatments were used, including; Copper oxychloride combined with Mancozeb; Orius (Tebuconazole); Rodazim (Carbendazim); Alternation of fungicides (Tebuconazole and Carbendazim); Farmer practice and phyto-sanitation (removing of infected plant parts). In 2017A, an extra treatment included a no intervention control. Treatments were laid in a completely randomised design with farms used as 
replicates. The experimental plots contained nine plants $(3 \times 3)$. Carbendazim alternated with tebuconazole had the lowest pseudocercospora fruit and leaf spot disease progress (49.4\%) followed by tebuconazole (49.7\%) and carbendazim $(50.2 \%)$. Farmer practice showed the highest level of disease $(53.1 \%)$, while in $2017 \mathrm{~A}$, carbendazim had the lowest pseudocercospora fruit and leaf spot progress $(40.7 \%)$, while the no intervention control had the highest (56.6\%). Carbendazim, and Carbendazim alternated with tebuconazole are the most effective disease management options for reducing disease in citrus orchards in Teso reducing citrus disease by between 31 and 49\%. In 2017A, where there was no intervention there was increase in disease of $24.2 \%$ instead of a decline.

Challenges Associated with Biocontrol in Turfgrass

J. P. KERNS, North Carolina State University, Raleigh, NC, USA

Turfgrass managers view biological control as a desirable alternative treatment for disease as it can be used to extend or augment fungicide efficacy while reducing the amount of pesticide in the environment. The most common biological control strategies involve applying microbial inoculants or using organic amendments to encourage suppressive soils. Numerous products are available and declare fantastic results against various turfgrass diseases. Yet, empirical evidence in turfgrass systems is limited and the data that does exist does not paint a promising picture for biological control in turf. The most difficult obstacle to effective bio-control in a turfgrass system is maintaining adequate populations of the microbes in turfgrass soils. Turfgrass systems, despite claims to the contrary, have immense microbial populations even with pesticide and synthetic fertilizer inputs. Many laboratory studies indicate that microbial inoculants must remain well above 1 million cells per gram of soil in order to be competitive with plant pathogens. Many products are developed for traditional agriculture and vegetable production and microbial populations are significantly lower in these systems when compared to a turfgrass system. Thus, products from traditional agriculture are not effective in turf systems. Bio-control products or amendments developed specifically for turfgrass systems can be effective if the amount of microbes are sufficient to compete in turfgrass soils. This talk will cover the history of fungicide development, the development of biological control in turfgrass systems, the current knowledge surrounding microbial populations and composition in turfgrass soils and current products that demonstrate promise for the future.

The American Phytopathological Society: A century plus ten years young R. J. COOK, Washington State University, Bothell, WA, USA

The decision to form what became the American Phytopathological Society (APS) was made by six members of the American Association for the Advancement of Science (AAAS) that met during a meeting of AAAS held in December 1908 in Baltimore. In a summary of their deliberations, the ad hoc committee concluded that such a society may be an "invaluable aid in promoting the future development of this important and rapidly growing subject in America," and envisioned that "its influence may be made of international importance." Today, one third of the 4,500 members of APS live outside the U.S. Phytopathology began in 1911 as a bimonthly journal and became a monthly journal in 1918. In 2017, APS added Phytobiomes as its fifth monthly and first open access journal. The publication of five journals would not be possible without construction of a headquarters building in 1972, shared with the American Association of Cereal Chemists and later expanded. This building with its paid professional staff also made possible the formation of APS Press in 1984, which assumed responsibility for the compendium series, books and other non-journal publications and resources. Another milestone was the establishment in 1994 of a six-member National Plant Pathology Board for the purpose of meeting with members of the executive and legislative branches of the federal government on matters of policy and funding. This board has since become the Public Policy Board with a paid professional consultant, providing APS with possibly the most respected and effective presence in Washington DC of any scientific society representing the agricultural and plant sciences.

Banana Xanthomonas wilt is primarily spread by lance flies in the genus Silba through banana inflorescence in Ethiopia B. GETAHUN, D. Zeleke, Wolaita Sodo University, Sodo, ETHIOPIA

Banana Xanthomonas wilt (BXW) caused by Xanthomonas campestris pv. musacearum (Xcm) is an important disease of banana. Insects feeding on wounds resulted from abscission of infected flower and/or bract can possibly transmit the bacterium to healthy plants. This study was conducted to identify insect vectors and floral morphologies attributed to inflorescence infection on banana plants. Insects were collected and identified by genus from flowers of Pisang awak 'Abesha muz' and Cavendish genotypes in fields across southern Ethiopia in 2016. The presence of Xcm on insects was determined by isolating bacterial colonies in semi-selective media. Infectivity tests were conducted by inoculating Xcm suspensions on flowers and pseudostems of 30 plants of each genotype in field and green-house, respectively. Lance flies in the genus Silba were frequently associated with Xcm feeding on wounds from which the male flower and bract had abscised. Inoculation of these sites with Xcm produced infection on Pisang awak and 'Abesha muz'. Cavendish plants with persistent bracts and flowers apparently, however, remained healthy. When inoculated at the pseudostems of plantlets, BXW was produced regardless of banana genotypes. Manifestation of the first symptom through inflorescence inoculation took 40 days, exceeding by far the 60 days record on plantlets inoculated on pseudostems. This study demonstrated that the spread of BXW primarily occurred through the male flower wounds by insects.

Plantwise: As a source of intelligence on emerging disease in developing countries including Asia M. CHAUDHARY (1), R. Reeder (2), W. Jenner (3), K. Cameron (4), (1) CABI, New Delhi, INDIA; (2) CABI, Egham, UNITED KINGDOM; (3) CABI, Delemont, SWITZERLAND; (4) CABI, Wallingford, UNITED KINGDOM

Agricultural extension systems often poorly resourced to deal with the demand for information has highly variable ratios of public extension personnel to farmers as seen in Ethiopia from 1:476 to 1:5000 in India. The lack of targeted information impacts farmers' abilities to identify and effectively manage new pests and diseases. Filling in this gap Plantwise programme operates through more than 200 local partner organisations in 33 developing countries has network of more than 2300 established plant clinics operated by over 6,800 trained plant doctors reaching out 9.8 million farmers with recommendations in a form of written paper or digital on smart phone, tablet or computer. With more than $50 \%$ of the clinics now being empowered by technology over 10 countries with 30,000 records have E-plant clinics. This data held within a secured Plantwise Knowledge Bank can be analysed to assess a number of issues also pest surveillance for new pest outbreaks. This is not an explicit function of the plant clinics, but is achieved as by-product of farmers seeking assistance. Monitoring of pest out breaks is further enhanced by the use of social media by sharing of information through 'self-help' groups on mobile devices. The volume of message exchange is substantial and growing, with over 12,000 messages sent between groups in 2016 alone. This has proved to be highly instrumental in the early detection of invasive pests as in Asia, the banana skipper (Erionota torus), a new pest was identified triggered quick action from NPPO preventing considerable loss to the banana industry. Another potential use for the clinic data is pest forecasting providing pest risk predictions for a preventive action. The Pest Risk Information Service project aims to do this by creating a pest risk forecasting system based on earth observation data and ground truthing through the Plantwise clinic data. 
Fungicide sensitivity study of European Zymoseptoria tritici populations using large scale phenotyping and targets-based amplicon sequencing R. Frey (1), S. Widdison (2), G. Scalliet (1), H. Sierotzki (1), F. Walder (1), S. TORRIANI (1), (1) Syngenta Crop Protection, Stein, SWITZERLAND; (2) Syngenta Ltd, Bracknell, UNITED KINGDOM

Zymoseptoria tritici, the causal agent of Septoria tritici blotch is the most severe pathogen of wheat worldwide. Sustainable management of $Z$. tritici and other wheat diseases is mainly relying on correct fungicide spray programs. Monitor evolution and variation in fungicide sensitivity of pathogen populations is essential to provide accurate recommendation and it is considered by Food and Agriculture Organization of the United Nations (FAO) an integral part of the integrated pest management strategy. Z. tritici evolved target site resistance mechanisms to the major fungicide classes currently available (QoIs, DMIs and SDHIs). Resistance alleles to QoI, DMI and SDHI have been described in target genes $c y t b, c y p 51$ and $s d h B / C / D$, respectively. Some Z. tritici isolates might also express weak multidrug adaptation mainly associated to overexpression of the major facilitator gene $m f_{s} 1$. In this study we describe the results from the correlation between a high throughput genotype approach based on an Illumina amplicon sequencing and the fungicide sensitivity phenotype of 384 single spore field isolates collected from 11 European countries in 2016 toward two SDHIs, seven DMIs and one multisites. A deep understanding of geographic distribution and fungicide cross resistance profile of the most relevant resistant genotypes as well as the distribution of multiple resistances is essential to frame the impact to disease control and to propose sound new anti-resistance strategies.

A sneak peek into the citrus defense response affected by Candidatus Liberibacter effectors

S. Basu (1), R. Rabara (1), L. Huynh (1), S. ZHANG (1), J. Velásquez Guzmán (1), H. Nguyen (1), Q. Shi (2), G. Hao (2), E. Stover (2), G. Gupta (1), (1) New Mexico Consortium, Los Alamos, NM, USA; (2) U.S. Horticultural Research Laboratory, USDA-ARS, Fort Pierce, FL, USA

Huanglongbing (HLB) disease (a.k.a. Citrus greening), caused by Candidatus Liberibacter spp., is the most devastating disease of citrus. As in other bacterial pathogens, Liberibacter secretes effectors that play important roles in disease onset and progression. At present, however, the molecular details of interaction between Liberibacter effectors and host citrus targets remain unresolved. Here, we describe the identification of two putative effector proteins (designated as $\mathrm{LasP}_{235}, \mathrm{LasE}_{3}$ ) with a eukaryotic nuclear localization signal (NLS) in the prophage region of the Liberibacter asiaticus (Las) psy62 genome. The purified recombinant protein was used in pull-down assays with total protein from both healthy and Las-infected citrus leaves. The interacting complexes were subsequently purified using TALON affinity chromatography, and submitted to LC MS/MS, in order to identify citrus proteins that are unique to the healthy, infected, as well as several proteins that were expressed in both the healthy and infected samples. Among the identified interactors, we selected Aspartyl protease (AP), glycosyl hydrolases, LTP (lipid transfer proteins) and SOD (superoxide dismutase) for further validation. Using a similar approach, we also identified Kunitz-type Trypsin Inhibitor Protein (KTI), Asocorbate Peroxidase, Alcohol Dehydrogenase and Photosystem II proteins. Further biochemical assays, including characterization of AP, KTI, SOD and hydrolases, antimicrobial activity of LTPs, etc., in presence and absence of effectors, will be presented in detail. Our understanding of the mechanism of between Liberibacter effectors and citrus proteins should lead to the development of much-needed HLB therapies.

Mycotoxin contamination in maize is controlled by oxylipin signals

M. V. KOLOMIETS (1), P. Battilani (2), E. J. Borrego (1), M. Reverberi (3), A. Lanubile (2), V. Scala (4), C. Falàvigna (5), C. Dall'Asta (5), J. Bennett (1), Y. S. Park (1), (1) Texas A\&M University, College Station, TX, USA; (2) Università Cattolica del Sacro Cuore, Piacenza, ITALY; (3) Sapienza University, Roma, ITALY; (4) Consiglio per la Ricerca in Agricoltura e l'Analisi dell'Economia Agraria, Roma, ITALY; (5) University of Parma, Parma, ITALY

Mycotoxins are the most potent natural toxins and carcinogens that adversely affect diverse farm animals, poultry and humans. In corn, the major mycotoxin producing fungi are Aspergillus flavus and Fusarium verticillioides. Plant and fungal oxylipins are oxygenated fatty acids produced by diverse oxygenases including lipoxygenases (LOX). Plant oxylipins such as jasmonic acid (JA) function as signals in regulating defense. In fungi, oxylipins are potent regulators of mycotoxin biosynthesis and sporogenesis. Evidence will be presented showing that in addition to the major role of JA in defense against $F$. verticillioides, a range of understudied plant oxylipins may facilitate the pathogenic process while other oxylipins act to induce defense by upregulating JA biosynthesis. Supporting the hypothesis that oxylipins of both plant and fungal origin are engaged in the signaling cross-talk, analyses of $F$. verticillioides oxylipin mutants uncovered their dual role in both promoting pathogenesis and acting as elicitors of plant defense response. Overall, the study has identified several LOX genes required for resistance to $F$. verticillioides as well as a gene mediating susceptibility to fungal colonization and stimulating toxin production.

Potential biological control of the invasive Ailanthus altissima (tree-of-heaven) in Virginia using naturally occurring Verticillium wilt fungi R. K. BROOKS, A. Baudoin, S. Salom, Virginia Tech, Blacksburg, VA, USA

Ailanthus altissima (tree-of-heaven) is a difficult-to-control invasive tree that is now found in all continents but Antarctica. Research in Pennsylvania, USA has shown that two species of naturally occurring Verticillium wilt fungi may be able to act as biological control agents against this tree. To determine the effectiveness of these fungi outside of Pennsylvania, 24 plots dominated by A. altissima were located throughout Virginia, USA. A randomized complete block design was used to test 4 conidial suspension treatments ( $V$. nonalfalfae, $V$. dahliae, a 1:1 ratio of both, and water). In total, 524 A. altissima were stem-inoculated in May 2017 using a refined hack-and-squirt method. These plants were monitored at 0, 0.5, 1, 2, and 3 months post inoculation. The area under the disease progress stairs (AUDPS) for each plot was analyzed by treatment type and four representative site covariates (maximum temperature, average stem diameter at breast height, total rainfall, and average stem height). Independent of covariates, AUDPS differed significantly $(\mathrm{P}<0.05)$ between treatments, with both $V$. nonalfalfae and the combination of both fungi producing the highest AUDPS scores. Two covariates, maximum temperature and tree height, showed a significant $(\mathrm{P}<0.05)$ negative relationship with the AUDPS score, suggesting that some regional variation may occur. Further monitoring will give insight into how effective these pathogens may be throughout $A$. altissima's introduced range.

Progress in understanding of plant defense modulation by fungicides

J. KLEEMANN, K. Tietjen, Bayer AG, Monheim, GERMANY

It is a frequent field observation that fungicides exert beneficial effects in crop plants beyond their direct fungicidal action. Such crop strengthening effects, which are expressed as e.g. greening effects or stress tolerance, apparently can increase yield. Although in the past years advanced phenotyping methods have been developed that enable detection of these effects, molecular explanations are only fragmentary. Molecular biological data are presented that shed a light on the mechanisms of defense induction in crop plants by fungicides. 
In planta comparative transcriptomics and $\mathrm{Y} 2 \mathrm{H}$ to identify putative elicitors/suppressors of barley $r$ pg $4 /$ Rpg 5 -mediated stem rust resistance R. SHARMA POUDEL, S. Solanki, S. Shrestha, J. Richards, R. S. Brueggeman, Department of Plant Pathology, North Dakota State University, Fargo, ND, USA

The barley rpg4/Rpg5-mediated resistance locus (RMRL) confers resistance to many Puccinia graminis $\mathrm{f}$. sp. tritici (Pgt) races, including TTKSK. Rpg5 is the resistance gene at this locus encoding a typical NLR with a C-terminal protein kinase (PK) integrated sensory domain (ISD). To identify Pgt effectors/suppressors that elicit or suppress RMRL, 24 Pgt isolates with differential reactions were genotyped utilizing in planta RNAseq and 114K SNPs that result in predicted nonsynonymous amino acid changes were identified. Comparative analysis showed 17 genes associated with dominant RMRL virulence representing candidate suppressors of resistance. Host transcript analysis of a single barley line inoculated individually with the 24 virulent vs avirulent isolates showed virulent isolate specific down regulation of biotic stress responses, suggesting all Pgt isolates may contain a conserved effector that elicits RMRL responses by interacting with the Rpg5 ISD, yet virulent isolates harbor suppressors of resistance rather than the lack of a functional Avr protein. Y2H libraries constructed using RMRL avirulent isolate inoculated barley mRNA screened with the Rpg5 ISD as bait, identified a putative $P g t$ interactor that matches the criteria of a secreted effector and is highly expressed in all isolates. This suggests that it may represent the conserved effector that elicits Rpg5-mediated resistance responses. The validation of this putative effector and its function are underway.

Host mediated microbiome engineering for drought resistance in grasses M. JOCHUM, K. Mcwilliams, Y. K. Jo, Texas A\&M University, College Station, TX, USA

Rhizosphere microbial communities (rhizobiomes) impact plant growth and response to various stress. The goal of the study is to select a rhizobiome associated with alleviation of drought stress in the plant, using a multi-generation experiment known as host mediated microbiome engineering (HMME). Wheat seedlings were grown in bermudagrass rhizosphere composites collected from arid environments in west Texas, and exposed to a water deficiency. At the point in time when $90 \%$ of wheat seedlings exhibited drought symptoms, we selected rhizobiomes from the top $10 \%$ of the seedlings with delayed symptoms. Selected rhizobiomes were subsequently inoculated into new sterile soil where the next generation of wheat was planted. The aforementioned process was repeated for seven generations. At the conclusion of the experiment, seedlings growing in selected rhizobiomes could delay the onset of drought by 5 days compared to those growing in the unselected first generation. Selected rhizobiomes also exhibited a $17 \%$ increase in water retention, $43 \%$ increase in relative water content, $60 \%$ increase in daily water use efficiency, $27 \%$ increase in root length, $14 \%$ increase in shoot length, $36 \%$ increase in root dry weight, $24 \%$ increase in root surface area, and $25 \%$ increase in root tips compared to the sterile control. This project demonstrates the ability to manipulate rhizobiomes to act in a synergistic manner as a probiotic treatment for growing grasses under drought conditions.

Population studies of Pectobacterium atrosepticum: How it's shaping our view of seed-borne vs environmental sources of infection I. K. TOTH (1), E. Campbell (1), G. Cahill (2), J. Elphinstone (3), S. Humphris (1), G. S. Saddler (2), S. Wale (4), L. Watts (1), L. Pritchard (1), G. Harper (5), (1) James Hutton Institute, Dundee, UNITED KINGDOM; (2) Science and Advice for Scottish Agriculture (SASA), Edinburgh, UNITED KINGDOM; (3) Fera Science Ltd, York, UNITED KINGDOM; (4) Scotland's Rural College (SRUC), Aberdeen, UNITED KINGDOM; (5) Sutton Bridge Crop Storage Research (SBCSR), Spalding, UNITED KINGDOM

Blackleg disease, caused by bacteria from the genera Pectobacterium and Dickeya, can lead to devastating losses in potatoes throughout the production cycle. Over the last several decades, there have been substantial improvements in tuber storage, certification and handling, helping to control contamination of the pathogen once on tubers. However, understanding the processes by which the bacteria contaminate tubers in the field or during handling, and how we might minimise this contamination is still not fully understood. We are thus investigating when and where contamination occurs in the field, and how different conditions (i.e. weather or handling) may exacerbate this contamination and lead to increases in disease incidence. Our focus has been on the first field generation (the highest grade seed), which is free from the pathogen when planted but often goes on to show incremental increases in disease development over subsequent generations. If we can learn how contamination occurs and which interventions might reduce it, we hope to be able to better control the incidence of blackleg disease in future crops.

Dispersal of banana blood disease in Southeast Asia

J. RAY (1), V. Rincon-Florez (1), I. W. Mudita (2), J. Markus (2), S. Subandiyah (3), C. O’Dwyer (1), A. Drenth (1), (1) The University of Queensland, Brisbane, AUSTRALIA; (2) Nusa Cendana University, Kupang, INDONESIA; (3) Gadjah Mada University, Yogyakarta, INDONESIA

Blood disease of banana caused by Ralstonia syzygii subsp. celebesensis falls within phylotype IV of the Ralstonia solanacearum species complex. Symptoms include characteristic vascular staining, fruit bunches which appear outwardly healthy but are internally stained red/brown and rotten. Dispersal is associated with infected suckers, soil, tools and by insects. The disease was first observed in 1905 on Pulau Selayar a small island to the south of Sulawesi in Indonesia. It soon spread to the island of Sulawesi where a quarantine order in 1921 contained the disease. In 1987 the disease was detected in West Java from here it spread relatively quickly and was recorded in Bali and Sumatra (1994), Lombok, Sumbawa and West Papua (1999) and peninsular Malaysia (2013). We sought to determine the recent distribution through surveys. In Sumba, samples were collected from the symptomatic banana cultivars Pisang kepok (cooking ABB), Pisang ambon (sweet AAA) and Pisang barangan (sweet AAA). Bacteria were isolated from the samples and subsequently confirmed as Ralstonia syzygii subsp. celebesensis using molecular techniques. Initial analysis reveals a rapid spread from infested areas of $\sim 75 \mathrm{~km} /$ year. The severity of banana blood disease is significant as on Sumba the popular but highly susceptible Pisang kepok is no longer grown for export to other areas of Indonesia and subsistence farmers reported losses of $75-100 \%$.

Sexual propagation on barberry and its role in stem rust pathogen virulence and diversity Y. JIN, USDA-ARS Cereal Disease Laboratory, Saint Paul, MN, USA

In the past two decades, a limited number of races have been involved in wheat stem rust and stripe rust epidemics in East Africa and elsewhere. These highly virulent and aggressive races rose from obscurity, however. Investigations on a few known sexual populations of Puccinia graminis f. sp. tritici indicated that sexual cycles can generate diverse virulence recombinations, but only a few of which have the necessary virulences and aggressiveness enabling them to establish and thrive in the vast landscapes of cultivated hosts. There is little doubt that the Ug99 race group was originated in the highlands of eastern Africa, but how it came about is unclear. More complex virulence combinations in the Ug99 race group have been detected in recent years, indicating a continuous evolution. Our research has established that Berberis holstii, a native of East Africa highlands, is functional as the alternate host for P. graminis f. sp. tritici in Ethiopia. Its presence in proximity to wheat production in the highlands of East Africa may have had a role in the origin and evolution of the $\mathrm{Ug} 99$ race group. Berberis spp. also serve as the alternate host for the stripe rust pathogen ( $P$. striiformis). A number of Berberis spp., including $B$. holstii and $B$. vulgaris, is known to be susceptible to $P$. striiformis $\mathrm{f}$. sp. tritici in controlled inoculation. These species may have played a role in the origin of contemporary aggressive races that have been responsible for the sweeping epidemics around the world since the mid 
1980s. Finding ecological niches where active sexual cycles take place may offer some predictability on novel virulences that we may anticipate in the future.

Genetic diversity and development of improved diagnostics for Banana bunchy top virus (Nanoviridae: Babuvirus) in West and Central Africa A. ADEDIJI (1,2), R. Hanna (3), G. Atiri (2), P. L. Kumar (1), (1) International Institute of Tropical Agriculture, Ibadan, NIGERIA; (2) University of Ibadan, Ibadan, NIGERIA; (3) International Institute of Tropical Agriculture, Yaoundé, CAMEROON

Bananas (Musa spp.) are important staple foods worldwide, especially in sub-Saharan Africa (SSA). However, its cultivation is threatened by the banana bunchy top disease (BBTD) caused by Banana bunchy top virus (BBTV) which results in total yield loss. There is limited information on the genetic properties of BBTV in SSA countries where BBTD has recently been reported. Hence, this study was aimed at determining the genetic diversity of BBTV in West and Central Africa and to develop improved diagnostic tools. Banana leaves with BBTD symptoms were obtained from Nigeria, Benin Republic, Cameroon, Zambia and Gabon. Full genome of the six BBTV components were amplified and compared with other worldwide sequences. New polymerase chain reaction (PCR), loop mediated isothermal amplification (LAMP) and sap-based assays were developed to target the nuclear shuttle gene (DNA-N) and compared to existing rep-based protocols. All six BBTV components were successfully amplified in symptomatic banana leaves and showed close nucleotide (98-100\% identity) and amino acid (97-100\% identity) similarities among the isolates. Phylogenetic analyses clustered all isolates within the Pacific-Indian ocean sub-group. No evidence of recombination was found among the populations. Comparisons of newly developed primers with existing Rep-based primers showed DNA-N-based diagnostics to be ten times more sensitive in PCR (10 pg/ $\mu$ l) and LAMP (100 $\mathrm{fg} / \mu \mathrm{l})$ assays. Also, direct-binding PCR using sap-based extracts was ten times more sensitive $(1 \mathrm{ng} / \mu \mathrm{l})$ than ELISA test. Direct-binding LAMP was as sensitive as conventional PCR (100 pg/ $\mu$ l), thus eliminating the need for nucleic acid extraction. These results confirm BBTV entry into SSA via a common source as evidenced by a low genetic diversity. Improved detection will provide better options for BBTV management.

The first commercial bioherbicide based on a virus

R. CHARUDATTAN, Univ of Florida, Gainesville, FL, USA

Tobacco mild green mosaic virus strain U2 (TMGMV U2) elicits systemic necrosis and kills the noxious weed, Solanum viarum (tropical soda apple; TSA). In 2014, the U.S. EPA granted unrestricted FIFRA Section 3 registration for TMGMV U2 as a bioherbicide for TSA. It was the first registration of a virus as an herbicide active ingredient, and the product SolviNix ${ }^{\circledR}$ LC became the world's first virus-based bioherbicide. SolviNix is applied by postemergence spraying to control TSA in pastures and conservation areas. It controls TSA without harming grass, legumes intercropped in pastures, and native plants in and around pastures, or polluting soil or water. When properly applied to the foliage of actively growing TSA, SolviNix consistently kills TSA, including the roots. A scalable industrial manufacturing process has been developed to produce TMGMV U2 to meet variable market demands. As an unprecedented case, the registration of TMGMV U2 raised several specific concerns that needed to be addressed with empirical data or rational theory. Among them were whether TMGMV U2 was "widely prevalent" in the United States; its potential to spread "widely and uncontrollably" from the herbicidal use; the potential to be spread by farm machinery, cattle, and wildlife; its persistence in soil, water, and plant debris, and the risk to nontarget plants from the "moderately wide" host range of the virus. A synopsis of the data and arguments presented to justify the registration as well as the rationale for the label restrictions and the allowed application tools will be discussed.

\section{Research on Dickeya and Pectobacterium}

X. LI (1), B. Hu (2), J. van der Wolf (3), (1) Canadian Food Inspection Agency, Charlottetown, PE, CANADA; (2) College of Plant Protection, Nanjing Agricultural University, Nanjing, China, Nanjing, CHINA; (3) Wageningen University \& Research, Wageningen, NETHERLANDS

Species within the genera Dickeya and Pectobacterium cause significant crop losses because they are widespread and pathogenic on a wide variety of plant hosts. In potatoes, Dickeya/Pectobacterium cause blackleg and soft rot diseases which have become increasingly problematic in potato production. While Pectobacterium spp. have been known as causal agents of these diseases in North America since pioneer days, potato blackleg-causing Dickeya spp. were first discovered in the US in 2014. Given the trend in the spread of Dickeya spp. in Europe during the last 15 years, the US and Canada also need to be technically prepared for possible spread of new variants of Pectobacterium and Dickeya, such as D. solani. Although it has never been detected in Canadian crops, D. solani was isolated from hyacinth bulbs imported from Europe highlighting the potential pathway to NA. Comparative genomic analysis using NGS technology revealed differences among Dickeya and Pectobacterium strains congruent with demonstrated variations in pathogenicity and virulence in potato. For instance, an aggressive $P$. brasiliense subgroups is mostly isolated from potato in tropical regions such as Brazil and South Africa, while a less aggressive subgroup is isolated from potato in temperate regions. The two groups should probably be separated into different subspecies within $P$. brasiliense, on the basis of virulence associated with three major indels (insertions/deletions) in pathogenicity-related loci. Identification of genetic elements associated with pathogenicity and virulence of the blackleg pathogens has potential for enhancing the differentiation species and subspecies on the basis of their importance in agricultural crops.

Silicon enhances tolerance to abiotic and biotic stress W. L. ZELLNER, University of Toledo, Toledo, OH, USA

Silicon ( $\mathrm{Si}$ ) is a plant nutrient critical for stress tolerance. Most of our knowledge regarding the uptake and function(s) of Si comes from studies with specific monocots that accumulate high foliar Si concentrations (1-10\% Si dry weight). With this plant model system, Si has been shown to enhance defense as well as physically fortify cells for structural support. These physical responses are absent or reduced in plants, such as certain dicots, that accumulate less than $0.01 \% \mathrm{Si}$. Using tobacco, a low Si foliar accumulator, as a model system, Tobacco Ringspot Virus and copper (Cu) stress were shown to increase Si concentration in foliar tissue. Root Si concentrations were significantly higher than leaves, independent of the applied stress. Further studies indicated that root $\mathrm{Si}$ accumulation is not a significant source of soluble $\mathrm{Si}$ for leaves. In response to $\mathrm{Cu}$, foliar accumulation in tobacco was obtained from media-absorbed $\mathrm{Si}$ at the time of stress, as opposed to mobilized root stores. These data suggest a regulated transport system within tobacco. In addition, studies with tobacco transporters, NtNIP2;1, NtPIP1;1 and NtRT-TIP1, resulted in permeability to Si in a Xenopus oocyte system. NtNIP2;1 was also shown to be Si-regulated. Low foliar accumulators provide the opportunity to more clearly understand Si-mediated defense, independent of physical fortification. Future research will focus on characterizing transporters involved in cellular movement of the element as well as document the alleviation of stress by Si.

MicroRNA profiling of the whitefly Bemisia tabaci in response to feeding on tomato infected with Tomato yellow leaf curl virus D. K. HASEGAWA (1), M. Shamimuzzaman (1), W. Chen (2), A. Simmons (1), Z. Fei (3), K. S. Ling (1), (1) USDA-ARS, Charleston, SC, USA; (2) Boyce Thompson Institute, Ithaca, NY, USA; (3) USDA-ARS Robert W. Holley Center for Agriculture \& Health, Ithaca, NY, USA 
The whitefly Bemisia tabaci is a vector of viruses that infect food and fiber crops. Among the hundreds of viruses that are transmitted by $B$. tabaci, $90 \%$ of the species belong to the genus Begomovirus. To characterize whitefly regulatory mechanisms during Tomato yellow leaf curl virus (TYLCV) acquisition and transmission, we performed microRNA (miRNA) profiling of B. tabaci MEAM1 after feeding on tomato plants infected with TYLCV. A total of 160 miRNAs were identified, 68 being conserved and 92 novel miRNAs that were unique to B. tabaci. Interestingly, only two miRNAs were differentially expressed when whiteflies fed on TYLCV-infected tomato, compared to whiteflies that fed on uninfected tomato, and had predicted targets to Bta05482 (nuclear receptor) and Bta10702 (Very long chain 3R-3-hydroxyacyl-CoA dehydratase 2). We also mapped the miRNAs to another $B$. tabaci genome (MED) and identified six miRNAs that were unique to MEAM1. Lastly, to explore the relationship between miRNAs and gene expression, we referenced the miRNA data to transcriptome data, which was generated from the same pool of RNAs. Interestingly, approximately half of the miRNAs correlated inversely with their predicted target transcript expression (e.g., upregulated miRNA/downregulated transcript). Together, we link miRNA datasets with genomic and transcriptomic information to provide an in-depth analysis on the mechanisms of $B$. tabaci during virus acquisition and transmission.

Genetic engineering for resistance to Panama disease and Banana Bunchy Top J. DALE, Queensland University of Technology, Brisbane, AUSTRALIA

Commercial and small-holder banana production is often severely limited by one or more diseases. This is particularly evident with the most popular banana cultivar, Cavendish, which accounts for more than $50 \%$ of the world banana production and virtually all of the banana export market. Two of the most devastating diseases affecting Cavendish are Fusarium wilt or Panama Disease tropical race 4 (TR4) and bunchy top caused by Banana bunchy top virus (BBTV). Both these diseases are extremely difficult to control and both are continuing to expand their geographical ranges. We are using genetic modification to generate Cavendish lines that are resistant to these two diseases. For TR4 resistance, we previously identified an NB-LRR gene from a wild diploid banana, Musa acuminata ssp malaccensis which was differentially expressed in resistant and susceptible seedlings of that diploid. We transferred this gene to Cavendish and took a number of transgenic lines through a three year field trial. At the completion of the trial, one line remained completely disease free while three other lines had high levels of TR4 resistance. The level of resistance was correlated with the level of expression of the R gene. For BBTV, we have taken an RNAi approach targeting three different virus genes. After glasshouse screens in Australia, we have taken promising lines through to a field trial in Malawi. After 12 months, a number of lines remain disease free.

Diversity of decline-associated Phaeoacremonium species on woody hosts in South Africa

C. Spies (1), P. Moyo (2), F. Halleen (2), L. MOSTERT (2), (1) Agricultural Research Council, Stellenbosch, SOUTH AFRICA; (2) Stellenbosch University, Stellenbosch, SOUTH AFRICA

Nineteen Phaeoacremonium species are known in South Africa and have been reported from grapevines, pome and stone fruit trees, fynbos twig litter and arthropods. In other countries some of these Phaeoacremonium species are also known from hosts such as European olive, quince and willow that also commonly occur in South Africa. The aim of this study was to investigate the species diversity and host-range of Phaeoacremonium in the Western Cape Province of South Africa by characterising 158 isolates collected from 29 woody hosts. Phylogenetic analyses of combined actin and beta-tubulin datasets allowed for the identification of 32 species among the 158 isolates, including 14 new species and 3 known species that had not been recorded in South Africa previously. The new Phaeoacremonium species include P. album, P. aureum, P. bibendum, P. gamsii, P. geminum, P. junior, $P$. longicollarum, $P$. meliae, $P$. oleae, $P$. paululum, P. pravum, P. proliferatum, $P$. rosicola and $P$. spadicum. All previous records of $P$. alvesii in South Africa were re-identified as $P$. italicum, but both species were recovered during this survey. A total of 36 Phaeoacremonium species are now known in South Africa, more than double the number reported in any other country. The high number of Phaeoacremonium species reflects the diversity of indigenous flora of the Cape Floral Region, a biodiversity hotspot mainly situated in the Western Cape Province.

Co- and super-infection exclusion of Beet necrotic yellow vein virus and Beet soil-borne mosaic virus S. Liebe (1), J. F. Gil (2), E. Savenkov (2), E. Maiss (3), M. VARRELMANN (4), (1) Insitute of sugar beet research, Goettingen, GERMANY; (2) Swedish University of Agricultural Sciences, Department of Plant Biology, Uppsala, SWEDEN; (3) Leibniz University Hannover, Hannover, GERMANY; (4) Inst of Sugar Beet Research, Goettingen, GERMANY

Beet necrotic yellow vein virus (BNYVV) and Beet soil-borne mosaic virus (BSBMV) belong to the genus Benyvirus. Roots infected with BSBMV appear asymptomatic whereas light yellow vein banding, mottling and mosaic patterns can be observed on the foliar. In contrast, BNYVV induces a reduced root size with massive root proliferation and vein yellowing on the foliar. Since BNYVV and BSBMV share the same host-range and vector species; mixed infections occur frequently. Information on interaction between both viral species is limited. Therefore, the species interaction was studied in Nicotiana benthamiana and Beta vulgaris using fluorescently labelled cDNA full length clones. Viral populations of BYNVV and BSBMV remained spatially separated with only a few co-infected cells after simultaneous inoculation into $N$. benthamiana. Furthermore, plants that were initially infected with BNYVV could not be super-infected with BSBMV. Although both viral species showed a strong exclusion in mixed infections, reassortment experiments in Beta vulgaris demonstrated that viral RNAs of BNYVV can be replaced by BSBMV and vice versa resulting in a systemic infection with altered symptom development. Moreover, it was proven whether both viral species are able to form chimeric RNAs by recombination. The observation of spatial separation possess significant impact on plant virus evolution by acquisition of foreign sequences by means of recombination or reassortment.

Decoding high-throughput sequencing data to address different layers of plant virus diversity D. KUTNJAK, A. Pecman, K. Bačnik, I. Gutierrez Aguirre, N. Mehle, M. Tušek Žnidarič, M. Ravnikar, National Institute of Biology, Ljubljana, SLOVENIA

The unbiased nature and high data generation potential of high-throughput sequencing (HTS) technologies render them applicable to study viruses on multiple biological levels, from within the host to the ecosystem level. Starting within the host, HTS can be used to study the structures and dynamics of viral populations during virus evolution; using this approach, we characterized the structure of Potato virus Y (Potyvirus, Potyviridae) populations within plants and then tracked the changes in population structure of the virus during the experimental evolution in different host genotypes. We also applied HTS for detection of different viruses in plant samples and characterization of divergent viral strains (Potato virus X, Potexvirus, Alphaflexiviridae) and obscure viruses on new host plant species (Henbane mosaic virus, Potyvirus, Potyviridae). Finally, moving from single or few hosts to the ecosystem level, HTS can be employed for virome studies of environmental samples; water samples from a wastewater treatment plant were concentrated and subsequently we determined the viromes of those samples using HTS. A wide array of plant viruses was detected in these samples. Further infectivity assays using test plants confirmed the infectivity of some of the viruses detected using NGS approach. An overview illustrated with practical examples of the use of NGS across different levels of plant virus biodiversity will be discussed. 
Wheat blast management: Host resistance and fungicide protection

C. D. Cruz (1), P. SINGH (2), G. Cruppe (3), F. M. Santana (4), T. C. Todd (3), L. Calderon Daza (5), M. G. Rivadeneira Caballero (6), R. P. Singh (7), G. L. Peterson (8), H. J. Braun (9), B. Valent (3), (1) Purdue University, West Lafayette, IN, USA; (2) CIMMYT, E1 Batan, MEXICO; (3) Kansas State University, Manhattan, KS, USA; (4) Embrapa, Passo Fundo, BRAZIL; (5) Wheat Breeding Unit, Wheat and Oilseed Growers Association, Santa Cruz de la Sierra, BOLIVIA; (6) Centro de Investigación Agrícola Tropical, Santa Cruz de la Sierra, BOLIVIA; (7) CIMMYT, Mexico DF, MEXICO; (8) USDA ARS, Fort Detrick, MD, USA; (9) Cimmyt, Mexico, MEXICO

Wheat blast (WB), an emerging disease caused by the fungus Magnaporthe oryzae pathotype Triticum (MoT), has spread beyond South America to South Asia and threatens significant wheat production areas. Host resistance and fungicide application are two primary strategies to mitigate the effects of WB. There has been an intense search for sources of resistance to wheat head blast (WHB) since its discovery in 1985; however, identification of novel sources of resistance has proven difficult. To date, the only useful source is the 2NS/2AS translocation from Aegilops ventricosa. Global collaborative efforts are in place to establish field precision phenotyping platforms in Bolivia and Bangladesh and to collaborate with institutions across the globe on large-scale germplasm screening. Also, when weather conditions favor WHB development, fungicides are considered unreliable. Experiments conducted in 23 location-year environments in Brazil and Bolivia demonstrated that current chemical strategies to control WHB could provide some level of head blast control. Nevertheless, in some cases, that control could be limited and insufficient. We will present our understanding of the dynamics of MoT inoculum buildup, and discuss current breeding and fungicide timing strategies.

Microbial Species Interactions and Disease Suppression in the Phytobiome

L. L. KINKEL, Department of Plant Pathology/University of Minnesota, Saint Paul, MN, USA

Phytobiomes represent complex networks of interacting plant and microbial species. Our work explores the roles of plant community diversity, plant host, soil nutrient status, and fungal and bacterial species interactions in determining the pathogen-suppressive potential and composition of soil and foliar microbiomes. Our results show that rhizosphere Streptomyces associated with the same plant host are significantly more pathogen-suppressive and less niche-differentiated when the host grew in monoculture vs. within a high-diversity plant community. These data suggest that plant community diversity plays a critical role in determining the likelihood of antagonistic arms race coevolution vs. niche differentiation among sympatric soil populations, with significant implications for plant disease suppression. The power of fungal-bacterial coevolutionary species interaction in mediating inhibitory phenotypes is similarly evident in the observation that Fusarium are significantly better at inhibiting sympatric than allopatric populations of Streptomyces, and that inhibition is positively correlated with niche overlap between sympatric Fusarium and Streptomyces isolate pairs. This work illustrates the complex networks of species interactions that contribute to determining the pathogen suppressive potential of indigenous soil microbes.

Electrolized water for the control of postharvest decay of fruits and vegetables

A. IPPOLITO (1), S. M. Sanzani (2), (1) Department of Soil, Plant, and Food Sciences, University of Bari Aldo Moro, Bari, ITALY; (2) Department of Soil, Plant and Food Sciences, University of Bari, Bari, ITALY

During harvest, packaging, and storage operations, fruits and vegetables are exposed to various contaminations. A critical point during postharvest handling chain is washing, in which cross-contamination by specific pathogens may occur. Sanitation is of great importance to handlers, not only to protect commodities against postharvest diseases, but also consumers from foodborne pathogens. Use of proper sanitizers help preventing product contamination and maintaining the water free of pathogens. Various disinfectants are available, however, those most used, such as sodium hypochlorite, have limitations due to risks for environmental and human health. Among the alternatives to sodium hypochlorite, electrolyzed water (EW) has recently become a popular sanitizer in the food chain. EW was firstly developed in Russia for water decontamination and regeneration; then, it gained great interest in Japan and other Countries, for sterilization of utensils, meats, cutting boards, and recently, in livestock management and for the sanitation of the washing waters of fresh and minimally processed fruits and vegetables. Generation of EW, effect of electrolytes, advantages and disadvantages on its application, physical parameters, mode of action and efficacy on different commodities will be reviewed. Particular emphasis is given to the application of EW in the washing process of fresh fruits and vegetables and its integration into current practices of postharvest handling.

Suppression of PAMP-triggered immunity by coffee rust effectors may be attributed to their localization in the plant cell nucleus T. Maia, G. Marin-Ramirez, S. BROMMONSCHENKEL, Universidade Federal de Viçosa/Departamento de Fitopatologia, Viçosa, BRAZIL

Although studies have indicated that pathogen effectors may target a wide range of plant subcellular organelles in order to suppress host immunity, little is known about the function of effectors of rust fungi and their targets inside plant cells. Heterologous expression in Nicotiana benthamiana cells is an alternative approach to analyze Hemileia vastatrix candidate effectors (HvECs) due to the limited availability of molecular genetic tools to perform functional studies in this fungus. The present study aimed at performing a functional analysis of HvECs, through the evaluation of the ability of the candidate effectors to suppress PTI responses as well as by determining HvECs subcellular localization. Gene sequences encoding for 54 HvECs were individually cloned into pEDV6 vector (without signal peptide) and mobilized to Pseudomonas fluorescens EtHAn for transient expression in $N$. benthamiana. We found that 15 HvECs suppressed PTI with high reproducibility in different coinfiltration experiments with Pseudomonas syringae pv. tomato DC3000. We further designed a fluorescence-imaging-based screen to assess their subcellular localization. Confocal microscopy revealed that seven HvECs target the nucleus whereas only one effector accumulated in chloroplasts. Coffee rust effectors may manipulate nuclear host immunity in order to suppress plant defense response and promote susceptibility.

Field performance of a biotech potato with late blight resistance in Uganda M. GHISLAIN, International Potato Center, Nairobi, KENYA

The most devastating potato disease is late blight (LB) caused by Phytophthora infestans (Pi) and is still responsible for significant losses which may reach 30-75\% for small-scale potato farmers in sub-Saharan Africa. Added to the cost of fungicide, SSA farmers realize barely $40 \%$ of the value of their potential production. Breeding for LB resistance has been met with challenges of durability of resistance. Instead, we have transferred three resistance genes isolated from wild potato relatives ( $R B$, Rpi-blb2 from Solanum bulbocastanum and Rpi-vnt1.1 from $S$. venturii) into four cultivated potato varieties 'Desiree', 'Victoria', 'Tigoni' and 'Shangi' using genetic engineering. Transgenic events with extreme resistance to LB have been tested in confined field trials (CFT) in Uganda. All transgenic events tested grew normally and produced expected yield without any fungicide spray during three seasons. Remarkably, not a single lesion was observed at any stage of plant development. Fine molecular characterization of the T-DNA structure and insertion site for selected transgenic events from the variety 'Victoria' (known in Kenya as Asante) is underway. Multilocational CFT to conduct regulatory studies have already been initiated to assess risks to human (animal) and the environment. After this phase is completed, commercial release 
will be sought from national authorities. These biotech potatoes offer a durable cost-effective and environmentally-friendly solution to the control of LB disease in potato.

\section{The fluid dynamics of disease transmission: Drop impacts and transfer of pathogens} L. BOUROUIBA, Massachusetts Institute of Technology, Cambridge, MA, USA, MA, USA

Statistical correlations were reported between rainfall and crop disease outbreaks, suggesting that rain is a culprit in the dispersal of foliar pathogens. The mechanisms involved for dispersal of such pathogens and others in agricultural fields remain however poorly understood. In this combined experimental and theoretical study, we unveil the mechanisms at play when drops impact a range of infected surfaces. We discuss how fluid fragmentation is critical in shaping the patterns of contamination resulting from emission of pathogen-bearing droplets driving rain-induced epidemics. We discuss how these results interface with mechanisms driving disease spread at a range of scales in the field and between fields. These results are important to improve understanding of pathogen transmission and guide risk assessment and intervention strategies to mitigate crop contamination.

\section{History of Plant Pathology in Italy}

L. MUGNAI, A. Scala, G. Surico, DISPAA, University of Florence, Firenze, ITALY

Michelia, Commentarium Mycologiae Italicae is a journal published in 1877 and so called in honor of P.A. Micheli by P.A. Saccardo, who is known as "the Linnaeus of fungi" for having published the Sylloge fungorum omnium hucusque cognitorum (Collection of all the fungi so far known). Nearly 150 years previously, Micheli published Nova plantarum genera, a monumental work of 25 volumes in which he described nearly 900 different fungi, including species of Aspergillus, Botrytis and Puccinia, as well as the process of meiosis in basidia and the production of asci and ascospores. He also observed that fungi developed from the spores they produced. This work, published in 1729, is considered to be the birth of mycology worldwide. Before 1860s, many Italian scientists can be remembered, such as G. Targioni-Tozzetti and F. Fontana who contributed to the definition of the aetiology and early infectious phases of some wheat rusts. Then coinciding with Italy's political reunification (1861-1870) various Research Institutions began to be founded such as the Regio Istituto Forestale di Valle Ombrosa in Florence (1869), the Scuola Superiore di Agricoltura in Portici-Naples (1872), the Regia Stazione Vegetale in Rome (1887), all of which supported research and teaching activities of Italian Universities. Today, Italian Plant Pathology is highly developed and has enjoyed the foresight of many scientists who in the 1950s and 1960s layed the foundations for modern Plant Pathology.

\section{Understanding Phytophthora infestans populations at local and global scales}

D. COOKE (1), A. Lees (1), G. Kessel (2), D. Andrivon (3), P. Lassen (4), J. Grønbech Hansen (4), (1) James Hutton Institute, Dundee, SCOTLAND; (2) Wageningen Plant Research, Wageningen, NETHERLANDS; (3) National Institute for Agronomic Research, Le Rheu, FRANCE; (4) Aarhus University, Tjele, DENMARK

Late blight, caused by Phytophthora infestans, continues to threaten potato and tomato crops on a global scale. The timing and effectiveness of blight management depends on the pathogen's response to the environment, primary inoculum type, virulence to host resistance and sensitivity to fungicide active ingredients. The evolving $P$. infestans population requires management approaches that are tailored to the local population. The need to track the pathogen prompted the Euroblight consortium to collect data on the diversity of $P$. infestans, analysed with simple sequence repeat (SSR) genetic markers. Surveys of late blight infected crops by collaborators from 2013-2016 has resulted in over 5200 geo-tagged samples from 34 European countries. Standardised protocols and a single data format has allowed a flexible and powerful isolate database, associated analysis tools and a mapping interface to be built. A complex population structure is observed with 70 to $80 \%$ of the population dominated by a few widely disseminated clonal lineages; see maps at www.euroblight.net. The SSR diversity within lineages such as EU 13 A2 has been tracked over time and highlights the local spread of some sub-clonal variants. In contrast to the clones, $20-30 \%$ of the sampled European population comprises genetically diverse pathogen populations consistent with local, ephemeral oospore-derived sexual populations. Analysis via an interface with poppr, an R-based population genetics application appropriate for such mixed ploidy and mating system data, is allowing insights into the diversity of the potato late blight pathogen in Europe. Euroblight partners collaborate widely with other regional blight networks that have adopted our approach.

Molecular evidence of insect vector manipulation by a plant virus

J. WILSON (1), P. Pinheiro (2), Y. Xu (1), Y. Zheng (3), A. R. Rebelo (3), S. Fattahalhosseini (3), A. Kruse (1), R. Santos Dos Silva (3), Y. Xu (3), J. Giovannoni $(3,4,5)$, Z. Fei $(1,3)$, S. Gray $(1,6)$, M. Heck $(1,3,6)$, (1) Section of Plant Pathology and Plant-Microbe Biology, Cornell University, Ithaca, NY, USA; (2) Embrapa Rice and Beans, Santo Antonio de Goias, BRAZIL; (3) Boyce Thompson Institute, Ithaca, NY, USA; (4) Plant, Soil and Nutrition Research Unit, USDA-ARS, Ithaca, NY, USA; (5) Section of Plant Biology, Cornell University, Ithaca, NY, USA; (6) Emerging Pests and Pathogens Research Unit, USDA-ARS, Ithaca, NY, USA

The vector manipulation hypothesis proposes that plant pathogens manipulate their insect vectors to enhance their spread. Myzus persicae transmits several plant viruses, including the persistent, circulative Potato leafroll virus (PLRV). We suspected that PLRV may manipulate the aphid's antiviral immune response and tested our hypothesis using small RNA (sRNA) sequencing. We discovered that production of sRNA in the aphid is changed when aphids fed on PLRV-infected plants. In particular, PLRV altered the antiviral immune response of M. persicae to an aphid-infecting virus, Myzus persicae densovirus (MpDNV), as evidenced by an abundance of unusually long sRNA against MpDNV, a reduced number of 22-mers, and a higher titer of MpDNV when compared to aphids fed on uninfected plants $(\mathrm{p}=0.02)$. Feeding on plants only expressing P0, the PLRV silencing suppressor protein, recapitulated the increase in MpDNV titer in aphids, suggesting that P0 is the viral protein responsible for this effect. We hypothesize that this manipulation may have evolved to enhance the plant-to-plant spread of the virus. Winged aphids showed higher and more variable MpDNV titer compared to wingless individuals, establishing a connection between MpDNV and wing production, as observed for a densovirus in another aphid species. We hypothesize that the increase of MpDNV titer in aphids mediated by PLRV may result in an increase in the production of winged morphs and hence, long-distance virus dispersal.

Molecular mechanism of resistance to CAA and OSBPI fungicides in Phytophthora capsici and $\boldsymbol{P}$. sojae X. LIU, M. Cai, J. Miao, C. Zhang, China Agricultural University, Beijing, CHINA

Oomycetes are among the most problematic group of disease-causing organisms in agriculture. Flumorph is a CAA fungicide that has high activity against oomycete pathogens. Sequencing analysis indicated that CesA3, which is one of the four cellulose synthase enzymes, contained three substitutions in the amino acid sequence in the resistant P. capsici isolates. Back-transformation confirmed that the F1073L, G1105A, V1109L substitutions were linked to CAA resistance, and each mutation could make different contributions to the resistance phenotype. Meanwhile, five stable mutant types were obtained on flumorph-amended media in $P$. sojae. Further research found that the stepwise evolution of Ces $A 3$ resulted in increasing 
resistance to CAA fungicides. The Type I mutants were found to have a single point mutation (I1027V) in CesA3 protein which is the target protein of CAA fungicides, while the other mutant types not only carried the I1027V mutation but also possessed a second mutation V1025L, G1020A, G1020S or Q992H, respectively. Site-direct mutagenesis using CRISPR/Cas9 technology completely validated the role of each mutation, both individually and in combination with I1027V, with the transformants exhibiting similar resistance patterns to the original mutants. Oxathiapiprolin is the first of OSBPI fungicides. In our previous study, point mutations of G770V and G839W in oxysterol binding protein-related protein-1 (ORP1) were detected in oxathiapiprolin-resistant $P$. capsici mutants. In the current study, the CRISPR/Cas9 system was used to verify the effect of these two point mutations on characteristics of $P$. capsici. Transformants containing heterozygous G770V and G839W mutations in PcORP1 showed high levels of oxathiapiprolin resistance. These results will be useful for the development of the anti-resistance novel OSBPI fungicides.

Management of soil suppressiveness against soil-borne diseases

L. TAMM (1), B. Thuerig (1), G. Bongiorno (1,2), J. Postma (2), J. G. Fuchs (1), T. Oberhänsli (1), (1) Research Institute of Organic Agriculture FiBL, Frick, SWITZERLAND; (2) Wageningen Plant Research, Wageningen, NETHERLANDS

Soils suppressive to soil-borne diseases have attracted the attention of farmers and researchers for decades and many suppressive soils have been described. Microorganisms and soil microbial communities involved in suppressiveness have been studied intensively, but the underlying mechanisms are still not well understood. Suppressiveness to soil- as well as air-borne diseases has been shown to be highly site-specific. We will review how suppressiveness can be influenced by agricultural practices. Suppressiveness destroyed e.g. by steam sterilisation of soils could only be partially restored on the short-term by re-inoculation of soils, and the success of re-inoculation depended on the soil matrix as well as on the inoculum used. Long-term management (e.g. conventional or organic management), tillage regime, and short- or long-term fertility inputs have the potential to alter soil suppressiveness, yet the effect of a particular practice is still difficult to predict. Application of biocontrol organisms such as Pseudomonads or Bacillus sp. can have a beneficial effect against particular diseases on the short-term, but they can rarely persist after introduction in natural soils. Application of suitable composts can often help to sanitize fields contaminated with soil-borne pathogens more efficiently than individual biocontrol organisms. An ongoing study tries to identify key microbial consortia responsible for the beneficial effect of composts to allow a targeted application.

\section{Co-expression of Bs2 and EFR Genes in Tomato Provides Effective Broad-spectrum Field Resistance Against Bacterial Wilt and Bacterial Spot} of Tomato

S. KUNWAR (1), F. B. Iriarte (2), Q. Fan (3), E. da Silva (1), L. Ritchie (2), N. Nguyen (4), J. H. Freeman (2), R. E. Stall (5), J. B. Jones (6), G. V. Minsavage Jr. (6), J. Colee (7), C. Zipfel (8), D. M. Horvath (9), J. Westwood (9), J. W. Scott (10), G. E. Vallad (11), S. Hutton (12), M. Paret (2), (1) University of Florida, Quincy, FL, USA; (2) North Florida Research and Education Center, University of Florida, Quincy, FL, USA; (3) University of florida, Quincy, FL, USA; (4) University of Maine, Orono, ME, USA; (5) University of Florida, Gainesville, FL, USA; (6) Department of Plant Pathology, University of Florida, Gainesville, FL, USA; (7) Department of Statistics, University of Florida, Gainesville, FL, USA; (8) The Sainsbury Lab, Norwich, UNITED KINGDOM; (9) 2Blades Foundation, Evanston, IL, USA; (10) Univ of Florida, Wimauma, FL, USA; (11) Gulf Coast Research and Education Center, University of Florida, Wimauma, FL, USA; (12) University of Florida, Wimauma, FL, USA

Field trials were conducted at two locations in Florida to evaluate transgenic tomatoes expressing the EFR gene from Arabidopsis thaliana, the Bs2 gene from pepper, or both $B s 2 / E F R$ for managing bacterial wilt caused by Ralstonia solanacearum and bacterial spot caused by Xanthomonas perforans. Expression of EFR or Bs2/EFR, in the susceptible genotype, Fla. 8000, significantly reduced bacterial wilt incidence (50-100\%) and increased total yield (57-114\%) relative to lines expressing only Bs 2 or non-transgenic Fla. 8000 control, although the marketable yield was not statistically affected. Following harvest, the surviving plants in the fields were assessed for colonization by $R$. solanacearum. There were no significant differences in the population at the lower stem. Interestingly, in the middle stem, no bacteria could be recovered from $E F R$ lines or $B s 2 / E F R$ lines, but viable bacterial populations were recovered from $B s 2$ and non-transformed control lines at $10^{2}-10^{5} \mathrm{CFU} / \mathrm{g}$ tissue. $E F R$ transgenic tomato lines were effective against several $R$. solanacearum strains isolated from the southeastern U.S. indicating utility in a broad geographical area. In all three bacterial spot trials, EFR and $B s 2 / E F R$ lines significantly reduced disease severity compared to Fla. 8000 (22-98\%). The marketable and total yield of Bs2/EFR were significantly higher than Fla. $8000(43-170 \%)$ in three the of four field trials. These results demonstrate the potential of stacking Bs2 and $E F R$ genes for durable field resistance to bacterial wilt and bacterial spot of tomato.

Azole fungicide resistance: Evolution on a rugged fitness landscape N. HAWKINS (1), B. B. Fraaije (2), (1) Rothamsted Research, Harpenden, UNITED KINGDOM; (2) Rothamsted Research, Hertfordshire, UNITED KINGDOM

Predicting and delaying the emergence and spread of resistance is one of the most pressing applications of evolutionary biology in medicine and agriculture. The concept of fitness landscapes, in which a lineage under selection will be driven up an adaptive peak, has previously been applied to cases of antibiotic resistance evolution. We apply the concept of fitness landscapes to the evolution of fungicide resistance in plant pathogens, and in particular to the case of target site resistance to azole fungicides in Zymoseptoria tritici. The azole target site CYP51 now has over 30 reported non-synonymous mutations, combining in at least 80 different haplotypes with up to nine mutations each. Multiple mutations can have interacting effects on fungicide resistance and enzyme function, and these epistatic effects produce a rugged fitness landscape. A detailed understanding of the interactions between multiple mutations, and the trade-offs between fungicide resistance, functional constraints and fitness penalties, is needed to improve the predictability of evolutionary trajectories under different fungicide use scenarios.

\section{Canola Resistance Breeding to Fight against Blackleg Caused by Leptosphaeria maculans} Y. CHEN, Cargill Inc, Aberdeen, SK, CANADA

Blackleg [Leptosphaeria maculans (Desmaz.) Ces. \& de Not.] is one of the major diseases in canola (Brassica napus L.) worldwide that can cause significant yield loss. Tight rotation imposes extreme selection pressure against cultivar resistance, resulting in shift in $L$. maculans races. This has lead to breakdown of the resistance and more severe disease epidemics across western Canada. The best risk management is the introgression of major gene $(\mathrm{R} / \mathrm{m})$ and multigenic sources of resistance into canola hybrids, along with integrated risk management to slow down the erosion of resistance and ensure sustainable yield in Cargill's Victory canola hybrids. The method is to evaluate L. maculans AvrLm allele frequency and identify unique sources of genetic resistance from Cargill gemplasm; fine mapping of the genes using 60K SNP genotyping array to find the gene-specific markers for markerassisted breeding; In-vitro assessment over near-isogenic lines carrying introgressed genes, followed by outdoor assessment of adult plant resistance; finally judicious deployment of resistant varieties and risk management strategies recommended to the growers. Stacked Rlm genes in combination with QTL haplotypes provide Cargill Victory hybrids with broad and durable resistance to the blackleg. The resistance in Cargill products has been in a leading position since 2010, indicating that this breeding strategy is effective. Monitoring the dynamics of AvrLm allele frequency in the pathogen 
population is a great tool in determining the usefulness of Rlm genes. Trait-specific markers are proving to be powerful tools in marker-assisted gene introgressions, selection in doubled-haploid (DH) breeding populations and hybrid evaluation.

Habitat suitability of Phytophthora palmivora using bioclimatic models

H. A. NAROUEI-KHANDAN, M. Ormsby, A. Herath, Ministry for Primary Industries, Wellington, NEW ZEALAND

Phytophthora palmivora is a ubiquitous plant pathogen with major impact on more than 1000 plant species in over 15 plant families, including many economic and environmental important plants. Predictive models which evaluate the risk of the pathogen establishment can be beneficial to regulatory agencies in non-infected areas. Two bioclimatic models, MaxEnt and CLIMEX were used to project the potential distribution of $P$. palmivora at a global scale and in New Zealand. Long-term climate data (temperature and precipitation) were acquired from the Worldclim website, and global occurrences of the pathogen were gleaned from different sources including online databases and personal communications. The pathogen occurrences in Australia were used as independent data to validate the model. The model projections were a very good fit to the current global distribution of the pathogen. Both models performed well on independent validation data, projecting the currently infested coastal areas in Eastern and Western Australia as highly suitable for pathogen establishment. In New Zealand, where the pathogen is not known to be present, both models projected the climate in North Island as being conducive to pathogen establishment. CLIMEX results indicated that the growth index in the North Island is the highest during January to March and the lowest from June to September. MaxEnt indicated annual mean temperature and precipitation of the wettest quarter as variables contributing the most to pathogen establishment. The results can provide information to alert decision-makers in non-affected areas to the potential need for measures to prevent pathogen entry or to prepare for possible incursions.

Collaboratively managing sudden oak death in California and Oregon using tangible landscape models D. Gaydos (1), R. Cobb (2), D. M. RIZZO (3), R. K. Meentemeyer (4), (1) Department of Forestry and Natural Resources, North Carolina State University, Raleigh, NC, USA; (2) Cal Poly State University, San Luis Obispo, CA, USA; (3) University of California, Davis, CA, USA; (4) North Carolina State University, Raleigh, NC, USA

Geospatial models allow users to explore the dynamics of complex socio-ecological processes occurring over large spatial and temporal scales, making them uniquely suited for disease management applications in forests. In many cases, however, models are developed without consideration of local stakeholder motivations or compelling user-friendly interfaces, fueling a knowledge-practice gap where better science has not necessarily lead to better management. Tangible Landscape, an open-source participatory modeling tool, has been designed to address this challenge by coupling a physical model of the landscape with a geographic information system. Tangible Landscape allows stakeholders to alter the landscape and instantly visualize management effects. We are using this modeling platform to engage stakeholders involved in sudden oak death (SOD) management in northern California and southwest Oregon. SOD is an extremely destructive, introduced disease which has killed millions of oak and tanoak trees in California and Oregon. We will discuss how to use Tangible Landscape as platform for fostering collaborative discussions and co-learning among diverse stakeholder groups who must consider different policies and intervention strategies, along with multiple dynamic trade offs, in order to manage SOD in their local environment.

Multiscale remote sensing of plant pathogens: Detecting and monitoring myrtle rust

R. HEIM (1,2), I. J. Wright (1), H. C. Chang (1), A. Carnegie (3), J. Oldeland (2), (1) Macquarie University, Sydney, AUSTRALIA; (2) Hamburg University, Hamburg, GERMANY; (3) NSW Department of Primary Industries, Parramatta, AUSTRALIA

Australia's natural and managed landscapes, dominated by the plant family Myrtaceae, are under threat from a devastating, invasive disease called myrtle rust (Austropuccinia psidii). Already, the lemon myrtle (Backhouse citriodora) essential oil industry has suffered yield losses up to 70 percent per year. Due to recurring infections, natural populations of several highly susceptible native plant species seem likely to go extinct. Currently, detecting and monitoring disease outbreaks is only possible by eye, aided by forecasting infection periods, including co-incident timing of spore release and host growth flush, using weather data. These methods can be resource intensive and sometimes unreliable. Fungicides can only be applied once clear disease symptoms are visible, which is wasteful, ineffective and resulting in high costs. However, optical remote sensing techniques are well known for objective and reliable automated diagnosis of plant diseases. Combined with advanced data analysis techniques, sustainable and targeted pest management systems can be developed. This study aims at establishing a proof-of-concept for monitoring and detection of myrtle must. At the ICPP 2018 in Boston, we would like to present results of our first sub-project where we could discriminate healthy and infected lemon myrtle trees with an accuracy of $95 \%$ based on their leaf spectral characteristics. In particular, we would like to present (i) the results of our classification of hyperspectral data collected at the leaf level; (ii) a self-designed myrtle rust-specific vegetation index and introduce and an approach to validate this index with multispectral aerial imagery, and iii) provide an outlook on upcoming projects, especially on data we collected for other plant species to extend our spectral library of myrtle rust specific signatures.

Identification of disease suppressive banana plant and soil microbiomes

P. DENNIS (1), H. Birt (1), A. Raghavendra (2), G. Waugh (2), H. Wang (2), E. Aitken (3), T. Gervacio (2), R. Orr (4), P. Nelson (4), J. Daniells (5), T. Pattison (6), (1) The University of Queensland, St Lucia, AUSTRALIA; (2) The University of Queensland, Brisbane, AUSTRALIA; (3) School of Agriculture and Food Sciences, The University of Queensland, Brisbane, AUSTRALIA; (4) James Cook University, Cairns, AUSTRALIA; (5) Department of Agriculture and Fisheries, South Johnstone, AUSTRALIA; (6) Department of Agriculture \& Fisheries, South Johntone, AUSTRALIA

Plant-associated microorganisms are known to strongly influence host fitness but are poorly characterised for bananas and the soils used for their production. We have characterised the microbiomes of: 1) 17 soil types representing c. $70 \%$ of the main banana production area of Australia; 2) 55 banana genotypes; and 3) multiple plant compartments using high throughput phylogenetic marker gene sequencing. In addition, we have ranked the suppressiveness of multiple soil types to Fusarium wilt (Fusarium oxysporum f. sp. cubense) and used a novel bait trapping technique to identify microorganisms that are attracted to compounds released by the pathogen. Consequently, we have identified a range of microorganisms that are associated with susceptibility and resistance to the disease. These findings will assist other projects that aim to identify on-farm management practices that encourage the establishment of Fusarium wilt suppressive banana plant and soil microbiomes under field conditions.

Fungicide resistance issues in scab pathogen Fusarium graminearum and DON contamination in wheat Z. MA, Institute of Biotechnology, Zhejiang University, Hangzhou, CHINA

Fusarium head blight (FHB) caused by $F$. graminearum (Fg) and several other Fusarium spp. is a devastating disease of cereal crops. In addition to the yield loss caused by the disease, the contamination of infected grains with mycotoxins poses a serious threat to human and animal health. Currently, one 
primary method for management of FHB is through the application of fungicides during wheat anthesis due to the lack of highly resistant wheat cultivars. In this presentation, we will review some of recent advances in i) monitoring fungicide resistance in Fg; ii) resistance mechanisms of Fg to several major classes of fungicides including benzimidazoles, demethylation inhibitors, and a novel compound phenamacril; and iii) effects of fungicide treatment on mycotoxin biosynthesis. Finally, we will also discuss the strategy for management of fungicide resistance in FHB.

Investigation into the secretion and localization of Ustilago hordei avirulence effector UhAVR1

A. P. MONTENEGRO ALONSO (1), G. Bakkeren (2), (1) University of British Columbia, Vancouver, CANADA; (2) Agric \& Agri Food Canada, Summerland, BC, CANADA

We previously identified avirulence effector UhAVR1 in Ustilago hordei, a basidiomycete fungus causing covered smut disease on barley. UhAVR1 is a 171 aminoacids protein with an N-terminal signal peptide (SP). Presence of resistance gene Ruhl in barley cv. Hannchen leads to a strong hypersensitive response upon infection, arresting pathogen growth, whereas cv. Odessa (ruhl) supports disease. To determine the virulence function of UhAVR1, localization and secretion studies were carried out. In Nicotiana benthamiana, using agroinfiltration, transiently expressed UhAVR1:eGFP mature peptide (-SP) was shown to localize to the nucleoplasm and cytosol where it was concentrated in small cytosolic bodies. When testing UhAVR1+SP:eGFP, no apoplastic localization was seen upon plasmolysis. Protein blots confirmed the production of full length fusion protein. Agroinfiltration in barley confirmed a cytosolic localization. In a novel secretion system, full length UhAVR1:mCherry was detected by protein blots when expressed from a constitutive strong Hsp70 promoter in haploid U. hordei cells, as well as in spent medium, indicating secretion. Infiltration in barley leaves of this Ustilago strain showed UhAVR1:mCherry fluorescence in cytosolic bodies in mesophyll cells. With Brefeldin A, secretion of UhAVR1:mCherry seemed unaffected, suggesting secretion through a non-canonical pathway. This would be the first report of non-conventional secretion of a basidiomycete pathogen effector.

Progress in sheath blight management in rice

U. S. SINGH, Intl Rice Research Inst - India Office, New Delhi, INDIA

Sheath blight disease, caused by Rhizoctonia solani, is the second most important rice disease worldwide after blast. It is particularly important when rice is grown intensively. In the lowland rice of tropical Asia average yield losses caused by sheath blight range from 5 to $10 \%$. Host plant resistance represents a key approach for disease management. However, in the case of sheath blight, only quantitative (partial) resistance can be expected in rice, given the biology and wide host range of the pathogen. In spite of lot of efforts, so far no rice variety with a high level of resistance is deployed in Asia. Resistance inducing chemicals that are able to induce broad disease resistance offer an additional option for the farmer to complement genetic disease resistance/tolerance and the use of fungicides. Strobilurin based molecules like azoxystrobin, trifloxystrobin, metominostrobin has also been reported to manage the disease effectively and eco-friendly way than other commercially available fungicides. Efficacy of several fungicide combinations such as azoxystrobin plus difenconazole, kresoxim methyl plus hexaconazole, fluxapyroxad plus epixiconazole, trifloxystrobin plus tebuconazole, flutolanil plus azoxystrobin, thiophonate methyl plus azoxystrobin, tebuconazole plus azoxystrobin, propiconazole plus azoxystrobin against sheath blight have been reported. Thifluzamide is a member of the carboxamide class of fungicides which interfere with fungal respiration via their inhibitory effect on succinate dehydrogenase within the tricarboxylic acid cycle has been reported effective in managing sheath blight of rice. However, in case of heavy rains for longer period fungicide alone may not solve problem particularly if crop canopy is dense.

Systematic characterization of ROS-responsive transcription factors in Verticillium dahliae C. TANG, C. Tian, Y. Wang, Beijing Forestry University, Beijing, CHINA

Verticillium dahliae is a soilborne fungus that causes plant vascular wilt disease, and poses a serious threat to hundreds of species, including numerous economically important crops. For successful invasions of host plants, fungi have deployed complex strategies to counteract harmful effects of reactive oxygen species (ROS) produced by plant. This study focused on elucidating the molecular basis of how $V$. dahliae regulates the oxidative stress response by characterizing the roles of ROS-responsive transcription factor orthologs, such as VdYap1, VdAtfl, and VdSkn7, in pathogenesis. Single and double deletion mutants of VdYap 1, VdAtfl, and VdSkn7 were generated and the mutants were functionally characterized. The results revealed that VdSkn7 contributes to microsclerotial development, virulence and stress responses, while VdAtfl is involved in the conidial production, and regulation of the activity of extracellular peroxidases and laccases, and virulence. Compared to either the single mutants or the wild-type strain, the double mutant (VdAtfl and $V d S k n 7$ ) exhibited significantly increased sensitivity to oxidative stress, as well as severe defects in conidiation and virulence. The mutant VdYap 1 also showed reduced responses to oxidative stress and a reduction in virulence. Transcriptomic and proteomic analysis were used to uncover the common and specific genes or pathways regulated by the three ROS-responsive transcription factors. Together, our results indicate that VdYap1, VdAtf1, and VdSkn7 have overlapping functions, with some very distinct differences, in modulation of the anti-oxidative response, microsclerotial formation, and ROS signaling for pathogenesis in $V$. dahliae.

Engineering Plant Immunity via CRISPR/Cas Systems M. MAHFOUZ, KAUST, THUWAL, SAUDI ARABIA

CRISPR/Cas systems confer immunity against invading nucleic acids and phages in bacteria and archaea. Recent studies have shown that CRISPR/Cas9 system is capable of targeting the genomes of DNA viruses and can interfere with viral activity and limit viral symptoms in planta, demonstrating the utility of this system for engineering molecular immunity in plants. CRISPR/Cas9 can efficiently target single and multiple viral infections and confer plant immunity. CRISPR/Cas13a (known previously as C2c2) is a class 2 type VI-A ribonuclease capable of targeting and cleaving single-stranded RNA (ssRNA) molecules of the phage genome. CRISPR/Cas13a has been shown to confer modest interference against RNA virus in plants. Here, I discuss the use of different CRISPR/Cas systems to engineer molecular immunity against DNA and RNA viruses in plants. I also highlight the potential challenges encountered when producing plants with engineered resistance to single and mixed viral infections.

An overview of challenges and changes in potato production and potato diseases in the United States and Canada A. O. CHARKOWSKI, Colorado State University, Fort Collins, CO, USA

The only known major pathogens that caused bacterial blackleg and soft rot of potato in North America used to be Pectobacterium atrosepticum and $P$. carotovorum. However, even early genomics methods demonstrated that multiple previously undescribed Pectobacterium species caused blackleg and soft rot. Available phylogenetic and genomic data suggests that additional species remain to be described in the United States. Over the past decade, multiple species capable of causing these diseases were identified and sequenced. In 2014, a blackleg outbreak caused by $D$. dianthicola was identified and this pathogen quickly spread to multiple potato-producing regions via seed potatoes. Two distinct $D$. dianthicola strains were identified in multiple 
states during outbreak, but other Dickeya species do not appear to play a major role in blackleg in North America. D. dianthicola was often found with Pectobacterium, with P. parmentieri appearing to be one of the most common co-occurring species. Changes in potato varieties and testing of new potato varieties may have contributed to the recent $D$. dianthicola outbreak. Improvements in seed lot testing, including a new PCR assays developed from genomic analyses have reduced blackleg incidence over the past two growing seasons. Seed potato certification data has provided some information on potato lines that tolerate blackleg or that are highly susceptible. Further screening material for resistance is difficult due to the high variability in disease tolerance caused by plant age and environment, so virulence assays that provide improved quantitative ratings of disease tolerance were developed.

Suppression of Pratylenchus thornei after sequences of resistant summer grain crops maximised production of an intolerant wheat cultivar K. OWEN (1), T. Clewett (1), K. Bell (2), J. Thompson (1), (1) University of Southern Queensland, Centre for Crop Health, Toowoomba, AUSTRALIA; (2) Department of Agriculture and Fisheries Queensland, Toowoomba, AUSTRALIA

When populations of the root-lesion nematode, Pratylenchus thornei, have been reduced by cropping sequences with resistant crops, it is unknown how rapidly they increase in subsequent susceptible, intolerant crops. Summer crop cultivars of sorghum, maize, sunflower, mungbean and soybean were grown on adjacent sites with $P$. thornei at i) low levels ( $<125 / \mathrm{kg}$ soil) following five resistant summer crops (cotton-sorghum-sorghum-sorghum-maize) or ii) at moderate levels (2500/kg soil) following a wheat-sorghum-wheat sequence. In the subsequent season, wheat cultivars with tolerance or intolerance to $P$. thornei were grown. There was no difference in biomass or grain yield of the summer crop cultivars between the two sites, but $P$. thornei increased substantially at the site with initially moderate levels after susceptible soybean and mungbean cultivars (12000-20000/kg soil). In contrast, at the initially low $P$. thornei site, populations increased slightly but remained $<250 / \mathrm{kg}$ soil with no significant difference $(P>0.05)$ between summer crop cultivars. This effect then impacted the next crop in the cropping sequence, where the grain yield of the intolerant wheat cv. Strzelecki was similar to the tolerant cv. Wylie at the low $P$. thornei site (mean yield $\sim 3650 \mathrm{~kg} / \mathrm{ha}$ ) but reduced by $49 \%$ at the moderate $P$. thornei site (mean yield 1890 $\mathrm{kg} / \mathrm{ha}$ ). Optimal crop sequences to reduce $P$. thornei take many years, but the subsequent benefits can expand cultivar choice for farmers.

Alternate hosts for the yellow rust pathogen and their role in generating new virulence Z. KANG, J. Zhao, Y. Tian, L. Wang, Northwest A\&F University, Yangling, Shaanxi, CHINA

Wheat stripe rust, caused by Puccinia striiformis f. sp. tritici (Pst), is a damaging disease of wheat in the world. Epidemics of the disease cause severe yield reduction. Use of wheat resistant cultivar is an economic, effective approach for control of wheat stripe rust. However, the resistance of wheat was often overcome by new races. Mutation, somatic recombination and sexual recombination were proven to cause virulence variation to produce new races in rust fungi. But, sexual cycle of $P$ st has not been known for centuries until recent discovery of barberry (Berberis) identified as alternate host for Pst. To date, many barberry species, native to China (mainly), USA, and European countries, have been identified as alternate hosts for Pst. Importantly, Pst samples recovered from rusted barberry species have been reported in China, but not in other countries. This indicated that Pst completes sexual cycle on barberry in nature in some regions of the world. Sexual hybridization of Pst can produce a high diversity in virulence either single race or between races, greatly higher than asexual reproduction. This can be explained high diverse of Pst population in some areas of the globe, especially in China and Pakistan. Barberry plays an important role in occurrence of stripe rust of wheat in China but not in other countries so far, indicating that function of barberry in generating new virulence for $P$ st and providing inoculums for wheat may vary in different countries.

A myosin 5 dsRNA that reduces the fungicide resistance and pathogenicity of Fusarium asiaticum

X. S. SONG (1), K. X. Gu (1), Y. Duan (2), Y. P. Hou (2), M. Zhou (2), (1) Nanjing agricultural university, Nanjing, CHINA; (2) Nanjing Agricultural University, Nanjing, CHINA

Fungal resistance to fungicides is a serious challenge in crop protection. Although strategies have been found to prevent the development of fungicide resistance, no strategy has been found to reduce such resistance once it has occurred. We demonstrate that the application of a Myo5 dsRNA, which interferes with the target gene of the fungicide phenamacril in Fusarium, decreased F. asiaticum resistance to phenamacril as well as plant resistance to F. asiaticum infection. The silencing of Myo5 also resulted in cell wall defects, life cycle disruption, and virulence reduction. Unexpectedly, the silencing lasted for only a short time in $F$. asiaticum unless the dsRNA was continuously supplied. After being treated with a mixture of Myo5-8 dsRNA and phenamacril, plants highly resisted phenamacril resistance strain infection. Myo5-8 dsRNA was found to be highly conserved among $F$. asiaticum, $F$. grammearum, F. tricinctum, and F. oxysporum, and induced sequence-specific RNAi activity in these Fusarium species but not in other fungi. Our findings suggest a new strategy for reducing fungicide resistance and for designing new fungicides, which is significant in controlling of pathogens which easily develop fungicide resistance or are lack of natural resistance and specific fungicide.

How molecular biology is streamlining weed biological control research L. MORIN, CSIRO Health and Biosecurity, Canberra, AUSTRALIA

Research into classical weed biological control has progressed significantly since the first introduction of an exotic pathogen for weed biological control in 1971. This paper will present a series of examples from Australian biological control programs that involved the release of fungal pathogens against weeds in order to illustrate the importance of molecular biology in streamlining research in this discipline. DNA-based molecular characterization of target weeds is regularly utilized to determine the weeds' genetic structure in the introduced and native ranges to understand better the possible invasion pathways. This provides invaluable information that guides searches for suitable pathogen strains in the native range, which will be highly adapted to the weed genotypes found in the introduced range. DNA sequencing of informative genomic loci is now routinely used to elucidate the taxonomy and life cycle of potential pathogen agents discovered. Further, when several pathogen strains are introduced to a new region for weed biological control, molecular characterization enables effective monitoring of the strains' establishment, persistence and possible evolution over time. The use of cuttingedge technologies, such as genome-wide polymorphic marker systems and next generation whole genome sequencing, in weed biological control will further enhance researchers' ability to answer questions that are key to the future success of biological control programs.

\author{
Evaluation of biological control agents for reduction of aflatoxin contamination in corn using biodegradable corn starch-based bioplastic \\ formulations \\ H. K. ABBAS (1), C. Accinelli (2), W. T. Shier (3), (1) USDA ARS BCPRU, Stoneville, MS, USA; (2) University of Bologna, Bologna, ITALY; (3) \\ University of Minnesota, College of Pharmacy, Minneapolis, MN, USA
}

Aspergillus species produce potent carcinogenic mycotoxins, aflatoxins, which contaminate maize and other crops, resulting in millions of dollars in annual agronomic losses. Biocontrol using competitive displacement of aflatoxigenic Aspergillus flavus by non-aflatoxigenic $A$. flavus strains such as 
K49 and Afla-guard ${ }^{\circledR}$ was an effective method for reducing aflatoxin contamination of maize in field studies conducted in the Mississippi Delta during the past decade. Current research is focused on improving delivery systems for these biocontrol agents. We have developed and evaluated under field conditions in Italy and USA three formulations of a biodegradable corn starch-based bioplastic that improve the effectiveness of the biocontrol agents in reducing aflatoxin contamination in corn. First, applying bioplastic granules inoculated with A. flavus K49 and/or Afla-guard to field soil reduced aflatoxin contamination up to $90 \%$. Second, spraying a liquid formulation to leaves, which results in the colonization of leaf surfaces with biocontrol $A$. flavus strains, reduced aflatoxin contamination in corn by $80 \%$ to $94 \%$. Third, film-coating seeds with bioplastic containing spores of biocontrol $A$. flavus strains before planting reduced aflatoxin contamination up to $85 \%$. These results suggest that applying biocontrol A. flavus strains in such bioplastic formulations may be useful for reducing aflatoxin contamination in corn, but additional research is needed to optimize conditions.

Role of cation concentration in pepper tissue in suppressing bacterial leaf spot severity B. DUTTA (1), R. D. Gitaitis (1), D. B. Langston Jr. (2), J. Kichler (3), S. Carlson (1), (1) University of Georgia, Tifton, GA, USA; (2) Virginia Tech, Suffolk, VA, USA; (3) University of Georgia, Moultrie, GA, USA

The phytopathogenic bacterium Xanthomonas euvesicatoria causes bacterial leaf spot (BLS) of pepper and has a worldwide distribution. Several models were developed from 2012-2014 that were based on how macronutrients, micronutrients and micronutrient ratios affect BLS severity. Factors used to select a model for validation included highly significant $P$ values, high adjusted $R^{2}$ values, low variance inflation factor $($ VIF) values $(<5)$, root mean square error, and Mallow's Cp, and high Akaike's information criterion correction values. In addition, salicylic acid concentrations and relative expression of non-expressor pathogenesis-related gene1 $(N P R I)$ and pathogenesis-related protein $1(P R I)$ in pepper tissues were also considered in model selection. A model (ECGA1) consisting of concentrations of copper, manganese and potassium and the iron to zinc ratio as independent variables was used for validation in three different commercial pepper fields in Georgia [Colquitt, Worth and Tift County]. When AUDPC values for two field sites (Colquitt and Worth Counties) in 2015 were pooled and plotted against ECGA1, predicted values for both sites resulted in a a highly significant relationship $(P=0.0001)$ with an R-square value of 0.92 . A significant relationship between observed AUDPC vs. predicted values was also observed in Tift County (2016) $\left(P<0.001\right.$; adj. $\left.R^{2}=0.98\right)$. Relative gene expression of both $N P R I$ and $P R I$ genes were significantly $(P<0.01)$ higher in peppers grown in predicted low risk sites compared to peppers from high risk sites in Colquitt, Worth and Tift counties. Although BLS severity fluctuated depending on environmental conditions, the data suggested that the level of risk at a particular location may be influenced by how macronutrient and micronutrient concentrations affect plant disease resistance genes in the SAR pathway.

Developing a phenotyping tool for disease resistance in trees using Fourier transform infrared (FT-IR) and Raman spectroscopy A. O. CONRAD (1), R. A. Sniezko PhD (2), L. Rodriguez-Saona (3), P. Bonello (1), (1) Department of Plant Pathology, The Ohio State University, Columbus, OH, USA; (2) USDA FS Dorena Genetic Resource Center, Cottage Grove, OR, USA; (3) Department of Food Science and Technology, The Ohio State University, Columbus, OH, USA

Non-native and invasive plant pathogens are continuing to threaten the health and productivity of trees in natural ecosystems and commercial plantations. Few options currently exist for managing diseases caused by these organisms following their introduction and establishment. Identifying and breeding trees for resistance is one important management strategy, although it can be hindered by the long life cycles of trees and the lack of rapid phenotyping methods. Therefore, the objective of this study is to develop a phenotyping tool for disease resistance using Fourier transform infrared (FT-IR) and Raman spectroscopy combined with chemometric analysis. To test this phenotyping tool, we are focusing on two diseases: root rot and canker of Chamaecyparis lawsoniana (Port-Orford-cedar), caused by Phytophthora lateralis, and white pine blister rust of whitebark pine (Pinus albicaulis), caused by Cronartium ribicola. Using chemometric analysis, including partial least squares regression and soft independent modeling of class analogy, we are evaluating whether disease susceptibility can be predicted within and across families from different geographic origins. Our ultimate goal is to develop a protocol for the analysis of fresh plant tissues that can be used to more rapidly phenotype trees and facilitate the identification, preservation, and breeding of disease resistant trees.

Sentinel nurseries and plantations, approaches to tackle invasive plant pathogens before they move from their area of origin: The study case of China

A. VANNINI (1), A. M. Vettraino (2), R. Eschen (3), (1) DIBAF-University of Tuscia, Viterbo, ITALY; (2) Univ Degli Studi Dell Tuscia, Viterbo, ITALY; (3) CABI, Delémont, SWITZERLAND

Recent disease outbreaks caused by alien invasive pathogens into European forests posed a serious threat to forest sustainability with relevant environmental and economic effects. Many of the alien tree pathogens recently introduced into Europe were not previously included on any quarantine lists, thus they were not subject to phytosanitary inspections. The identification and description of alien fungi potentially pathogenic to native European flora before their introduction in Europe, is a paramount need in order to limit the risk of invasion and the impact to forest ecosystems as well as the potential role as vector of exotic trees traded to Europe. To determine the potential invasive fungi, a sentinel plantation of ecological relevant EU species and a sentinel nursery of the most traded exotic trees to EU were established in China. The fungal assemblage associated with specimens was studied using biological protocols and high-throughput sequencing of the nuclear ribosomal internal transcribed spacer-1 (ITS 1). Taxa with probable Asiatic origin were identified and included plant pathogenic genera. These results indicate that sentinel plantations and nurseries may be a strategic tool to improve the prevention of bio-invasions.

\section{Wheat blast: Danger on the move}

C. D. CRUZ (1), B. Valent (2), (1) Purdue University, West Lafayette, IN, USA; (2) Kansas State University, Manhattan, KS, USA

Wheat blast, caused by Magnaporthe oryzae Triticum pathotype (MoT), has caused widespread losses in Brazil and Bolivia. The recent wheat blast outbreak in Bangladesh demonstrates the threat of global spread of this intractable and dangerous disease. Few resistance genes have been identified and fungicides have shown limited efficacy. A complete understanding of MoT ecology and wheat blast epidemiology is needed to develop more effective management strategies. Understanding the full potential for pathogen variability, including the role of sexual reproduction in the field, is critical. A small number of pathogen avirulence (AVR) genes block other host-adapted $M$. oryzae pathotypes from infecting wheat; potential AVR gene mutations leading to new host jumps remain a threat. The current status of wheat blast research and disease control strategies indicating similarities and differences to rice blast and gray leaf spot of ryegrass will be discussed. Critical knowledge gaps in wheat blast epidemiology and disease management are identified.

Using hyperspectral reflectance-based predictive models for early Phytophthora infestans and Alternaria solani detection in potato K. M. GOLD, I. Herrmann, P. Townsend, A. J. Gevens, University of Wisconsin-Madison, Madison, WI, USA 
Late blight of tomato and potato (Phytophthora infestans) continues to be one of the most challenging diseases to sustainably and proactively manage. Potato early blight (Alternaria solani) occurs annually in Wisconsin. Growers spend upwards of $\$ 19$ million to control these two destructive diseases. Our previous work established a non-destructive method of early late blight detection based on hyperspectral reflectance that can identify infected plants with $>85 \%$ accuracy $2-4$ days before visual symptoms appear during its biotrophic phase. Our objective was to use this methodology to distinguish latent $P$. infestans infection from latent and symptomatic $A$. solani infection. We conducted two growth chamber experiments using $P$. infestans, $A$. solani, coinoculation, and control, non-inoculated plants to identify significant wavelengths for detection and differentiation. We measured continuous visible to shortwave infrared reflectance (400-2500 nm) on leaves using a portable spectrometer with contact probe at 12-24 hr intervals. We could both detect and distinguish early blight from late blight infection with $>80 \%$ accuracy at $24 \mathrm{hr}$ post-inoculation, and upwards of $90 \%$ accuracy after $48 \mathrm{hr}$ postinoculation. Shortwave infrared wavelengths $(>1300 \mathrm{~nm})$ were important for disease detection and differentiation. Our results support the potential use of hyperspectral reflectance-based predictive models as tools for rapid, early, real-time detection of two important diseases of potato.

The game-changing impact of NGS in Plant Virology

T. CANDRESSE, INRA Bordeaux, UMR 1332 BFP, Villenave d'Ornon, FRANCE

In the past few years, the rapid development of high-throughput sequencing (HTS) technologies has impacted many research areas. In virology and, in particular, in plant virology, HTS coupled with bioinformatics have dramatically changed the way virus discovery, etiology efforts or viral population analyses are performed. Among the advantages of such approaches is that they offer for the first time the ability to perform the complete viral indexing of a plant sample without the need for any prior knowledge. Protocols for the efficient analysis of a variety of templates including siRNAs, virionassociated nucleic acids (VANA), double-stranded RNAs (dsRNAs), mRNAs or total RNAs, with or without rRNA depletion, are now available, together with efficient pipelines for the bioinformatics analysis of the huge volumes of sequence data involved. As a consequence, a wide range of novel plant viruses have been described, a first glimpse at phytoviromes has been achieved and some viral populations have been described with unprecedented precision and depth. These technologies have a huge potential in certification and for the improvement of the safety of the conservation and movement of plant materials. Still, HTS-based viral indexing has yet to be applied on a large scale in routine diagnostic settings with the need to meet challenges such as validation, comparison of sensitivity with existing techniques or implementation of quality controls.

\section{Bringing potato disease resistance traits to market in the U.S. N. CHAMPOURET, JR Simplot Co, Boise, ID, USA}

The most widely used French fry potato variety in America, the Russet Burbank, and the most popular chips variety, Atlantic, recently celebrated their 100 -year and 40-year anniversaries respectively. In crops where varieties change infrequently, biotechnology tools and traits can readily bring benefits to farmers and consumers. To this end, Simplot Plant Sciences began working within the potato genome to reduce limitations of the widely grown commercial potato. The result is the Innate ${ }^{\circledR}$ potato platform with a stepped approach of increasing trait value from generation to generation. Simplot Innate ${ }^{\circledR}$ technology permits the selective improvement of positive traits or minimization of negative traits without incorporation of foreign genes. The second generation of Innate ${ }^{\circledR}$ potatoes reduce blackspot bruise and asparagine (which reduces the potential for the formation of acrylamide), cold storage capability, and provide foliar late blight resistance against common North American strains. Therefore, Innate ${ }^{\circledR}$ potatoes are genetically engineered with traits appealing to potato growers, packers, processors, retailers and consumers. The talk will mainly focus on the late blight protection trait strategy, how it was selected, a description of benefits, and how to maintain its efficacy. I will also discuss how resistance genes can be combined in various desirable permutations to protect against pathogen populations and provide a preview of future disease traits.

Crop-specific sulfur management for optimizing productivity, quality and plant health

S. HANEKLAUS, Julius Kuehn-Institut, Institute for Crop and Soil Science, Braunschweig, GERMANY

The implementation of biological know-how into fertilizer practice is a major contribution to fully utilize potential crop yield and quality, and to strengthen crops against biotic stress. Sulfur Induced Resistance (SIR) is one constituent of the complex phenomenon of induced resistance (IR). S fertilization reduced the plant disease index (DI) for various host/pathogen relationships by 5 to $50 \%$ (greenhouse) and 17 to $35 \%$ (field experiments), for instance Verticillium wilt in cotton from 0.708 to 0.375 . Effective fertilizer rates can be higher than the physiological S demand and vary cropspecifically between 20 to $40 \mathrm{~kg} / \mathrm{ha} \mathrm{S}$ before winter and 50 to $100 \mathrm{~kg} / \mathrm{ha} \mathrm{S}$ during main vegetative growth in spring; a constant S supply over time provided best protection against soil-borne and foliar plant diseases. The magnitude and efficiency of SIR seems to be regulated by the external plant available $\mathrm{S}$ reserves and plant-inherent $\mathrm{S}$ pools and fluxes, whereby the strongest response to $\mathrm{S}$ can be expected when going from severe $\mathrm{S}$ deficiency to sufficiency. $\mathrm{S}$ fertilization increases plant $\mathrm{S}$ status, content/release of S-containing primary and secondary metabolites (cysteine, glutathione,

glucosinolates, $\mathrm{H}_{2} \mathrm{~S}$ and $\mathrm{COS}$ ). All components show either a direct fungicidal/fungistatic effect or are involved in the induced resistance response. Based on a profound and robust statistical procedure, critical S concentrations (severe deficiency, optimum supply, toxicity) have been calculated for Brassica crops, legumes, pastures, Poaceae and root crops. These are recommended for adjusting fertilizer rates in order to maximize productivity and quality as well as enhance resistance against plant diseases.

Innovative management strategies for Aspergillus spp. and Penicillium spp. on nuts D. SPADARO, DISAFA and AGROINNOVA, University of Torino, Torino, ITALY

In postharvest, chestnuts and hazelnuts can be affected by species of Penicillium, agents of blue-green mould, and some species of Aspergillus, able to produce mycotoxins. Several strains of Aspergillus spp. and Penicillium spp. isolated from the chestnut field and production chain were characterized. A. flavus resulted the dominant Aspergillus species. Forty percent of the Aspergillus spp. strains produced $\mathrm{AF}_{\mathrm{S}}$ in vivo, while most strains were pathogenic on chestnut. A total of 20 species of Penicillium spp. were identified, including P. crustosum, P. glabrum, P. bialowiezense, P. discolor and $P$. expansum. Around $70 \%$ of Penicillium spp. isolated were pathogenic on chestnut, while $59 \%$ of the strains could produce at least one mycotoxin on chestnut. A new HPLC-MS/MS method was developed to detect twenty metabolites produced by Penicillium spp. on nuts. A LAMP assay was developed for Aspergillus flavus, while an antibody-immobilized microcantilever resonators was developed for mycotoxin detection. Drying conditions of chestnuts and hazelnuts is a critical point to reduce Aspergillus spp. and aflatoxin contamination. Monitoring, prevention and control are the strategies to manage Aspergillus spp. and Penicillium spp. on nuts. Besides these strategies, traditional static hot air roasting and infra-red rays roasting, together with cold atmospheric pressure plasma could be used for nut detoxification from aflatoxins. 
Population Genetics Analysis of Pythium myriotylum and Pythium aphanidermatum in Japan

A. AUliANA (1), C. Borjigen (2), K. Otsubo (3), S. Fuji (4), A. Hieno (3), H. Suga (5), K. Kageyama (3), (1) United Graduate School of Agricultural Science Gifu University, Gifu, JAPAN; (2) River Basin Research Center Gifu University, Gifu, JAPAN; (3) River Basin Research Center, Gifu University, Gifu, JAPAN; (4) Akita Prefectural University, Akita, JAPAN; (5) Life Science Research Center, Gifu University, Gifu, JAPAN

The modern agriculture with hydroponic systems trigger the emerge of water borne pathogen. Phythium myriotylum and P. aphanidermatum are water borne pathogen that has wide variety of host plant and responsible for seedling rots, root rots, damping-off, root and stem-rot on many economically important crops. For better understanding of disease route and the genetic relatedness, the microsatellite markers for population genetics analyses for both pathogens were developed by genome sequence analysis. In total, six $P$. myriotylum primer sets with 29 alleles were used to analyze 82 isolates from 22 hosts plants and 20 prefectures. In the other hand, $11 P$. aphanidermatum primer sets with 49 alleles were used to analyze 206 isolates from 13 host plants and 14 prefectures. The dendrogram made by UPGMA algorithm for $P$. myriotlum revealed that the isolates were rather clustered by their host plants than the geographical origin. On the other hand, the opposite result was obtained for P. aphanidermatum, it clustered by the geographical origin rather than the host plants. In conclusion, the different species might take different route to disperse.

Biotechnological Strategies for Control of Banana Xanthomonas Wilt Disease

L. TRIPATHI, International Institute for Tropical Agriculture, Nairobi P.O. Box 30709, KENYA

Banana Xanthomonas wilt (BXW), caused by the bacterium Xanthomonas campestris pv. musacearum, is one of the most important diseases and considered as the biggest threat to banana production in east Africa. The disease affects almost all commonly grown banana cultivars. Economic losses of about \$2-8 billion have been reported over a decade in the east Africa. The disease starts with wilting of leaves or male bud and premature ripening of fruits leading to death of plant, rotting of fruits, and in severe cases, complete loss of plantation. Its rapid spread has endangered livelihood of millions of farmers, who rely on banana for food and income. Management of the BXW disease is a challenge due to continuous association of host and inoculum over a long period of time. BXW disease can be managed by following cultural practices such as cutting and burying of infected plants, restricting the movement of bananas planting materials from BXW affected to disease free areas, removal of male buds and the use of clean farming tools. Currently, there are no commercial chemicals, biocontrol agents or resistant cultivars available to control the pathogen. In the absence of known natural host plant resistance among banana cultivars, genetic engineering provides a cost-effective alternative technique to develop Xanthomonas wilt resistant banana varieties. Host plant resistance against pathogens can be enhanced by expressing resistance (R) genes, antimicrobial genes, or defense genes. The progress in developing transgenic banana resistant to BXW disease will be presented.

How where and when to control an established plant disease epidemic. Landscape-scale modeling of sudden oak death in California N. J. CUNNIFFE (1), R. Cobb (2), R. K. Meentemeyer (3), D. M. Rizzo (4), C. A. Gilligan (5), (1) University of Cambridge, Cambridge, UNITED KINGDOM; (2) Cal Poly State University, San Luis Obispo, CA, USA; (3) North Carolina State University, Raleigh, NC, USA; (4) University of California, Davis, CA, USA; (5) University of Cambridge, CAMBRIDGE, UNITED KINGDOM

We use mathematical modelling to analyse control of sudden oak death in California. Sudden oak death, caused by the oomycete Phytophthora ramorum, has killed millions of oak and tanoak in California since its first detection in 1995. Despite some localised small-scale management, there has been no large-scale control in California to date. However, much information from both basic and applied research has accumulated over the past two decades, and we now have a good understanding of $P$. ramorum epidemiology. Mathematical models have been developed which integrate this knowledge, providing us with the opportunity to use modelling to test "what if" scenarios concerning management. We therefore use the Californian epidemic as a case study to illustrate how modelling can address general issues for invading epidemics. How quickly must control start for it to be effective? When is an epidemic so large that effective control is impossible? How should local treatment be deployed around infected sites? How does this depend on the available budget and level of risk aversion? Which sites should be targeted for control when there is insufficient resource to treat all infected locations? How can expenditure on detection and control be balanced? What is the effect of a budget that changes over time? The epidemiological principles underlying these questions are important for control of all invasive plant pathogens.

Insights into the epidemiology and transmission of grapevine red blotch virus

E. CIENIEWICZ (1), K. L. L. Perry (2), A. Kruse (3), M. Cilia (4), M. Fuchs (1), (1) Cornell University, Geneva, NY, USA; (2) Cornell University, Ithaca, NY, USA; (3) Section of Plant Pathology and Plant-Microbe Biology, Cornell University, Ithaca, NY, USA; (4) Emerging Pest and Pathogen Research Unit, USDA-ARS, Ithaca, NY, USA

Red blotch is an emerging viral disease of Vitis spp. that threatens grapevine production. The causal agent, grapevine red blotch virus (GRBV) from the genus Grablovirus in the family Geminiviridae, is disseminated via infected propagation material and spread by at least one hemipteran insect vector- the three cornered alfalfa hopper (Spissistilus festinus). Our research is aimed toward understanding the factors mediating spread of GRBV by S. festinus. We modeled spatiotemporal attributes of a red blotch epidemic in a $V$. vinifera 'Cabernet franc' vineyard in California over three years. Spatiotemporal analyses demonstrated predominantly localized, within-vineyard spread. We also described the seasonal and spatial distribution of $S$. festinus and additional potential vectors of GRBV in the 'Cabernet franc' vineyard. In parallel, hypothesizing a circulative transmission mode, we characterized the temporal GRBV retention by $S$. festinus on a non-host, described the gut morphology, determined GRBV tissue tropism in $S$. festinus using fluorescence in situ hybridization, and detected GRBV in dissected hemolymph, guts, and salivary glands from viruliferous $S$. festinus by multiplex polymerase chain reaction. A comprehensive understanding of the epidemiology and ecology of GRBV is crucial for the development of optimal disease and vineyard management recommendations.

A multi-faceted approach to promoting the use of bio-pesticides in Nepal: Experiences from Integrated Pest Management Innovation Lab S. PAUDEL, Penn State University, University Park, PA, USA

Over the past several decades, crop protection in Nepal has relied heavily on synthetic chemical pesticides in absence of proper alternatives. Various government and non-governmental programs adopted and introduced biopesticides as a part of their Integrated Pest Management (IPM) programs, yet the adoption has been rather slow. Integrated Pest Management Innovation Lab (IPM IL) program in Nepal, takes an innovative approach working with private sectors to facilitate and strengthen supply chain of biopesticides while at the same time working with government research and extension services, value-chain developmental program and NGOs. 
Development of a rapid and sensitive ddPCR method for detection of Cytospora leucostoma in peach orchards

J. R. IBARRA CABALLERO (1), L. Tembrock (1), F. Zink (1), T. Gilligan (2), J. Stewart (1), (1) Department of Bioagricultural Sciences and Pest Management, Colorado State University, Fort Collins, CO, USA; (2) Animal and Plant Health Inspection Service, USDA, Fort Collins, CO, USA

Colorado peach production is ranked 6th highest in the U.S., accounting for $75 \%$ of fruit produced in Colorado and contributing greatly to the state's $\$ 41$ billion dollar agricultural industry. Peach production is threatened by changing environmental conditions and pest and disease problems. The main pathogen species causing losses in peach production is Cytospora leucostoma, which causes Cytospora canker disease. It is currently thought that $C$. leucostoma is spread via rainsplash, however dispersal by insects and wind has not been thoroughly studied. Study of the insect and wind dispersal mechanisms has been hampered by the low quantities of DNA present in these types of samples. Therefore a rapid and sensitive assay for detection of $C$. leucostoma will be essential for epidemiological studies of the disease. Herein we present the development of a ddPCR method that can selectively detect C. leucostoma, from its non-pathogenic congeners. Diagnostic genomic regions were selected using the assembled genomes of four $C$. leucostoma isolates and one of $C$. parasitica. Primers and probes were then designed to selectively amplify $C$. leucostoma, using ddPCR. These primers were then validated using field collected spore samples. This work will help identify dissemination mechanisms used by C. leucostoma, thereby supporting the development of best management practices.

Planthopper-transmitted tenuiviruses infecting rice in the Americas

W. CUELLAR, International Center for Tropical Agriculture (CIAT), Cali, Valle del Cauca, COLOMBIA

Rice hoja blanca disease (RHBD) has a significant economic impact in the rice-growing regions of the Americas where it is endemic. Initial field reports date back to 1935 from the department of Valle del Cauca in Colombia. Then in the mid-1950s, it emerged in the state of Florida, threatening rice production in the southeastern USA. At the same time, wild grasses were identified as possible alternate hosts of the disease and symptoms including Echinochloa and Brachiaria hosts. Since then, RHBD has been reported affecting rice plantings in Brazil, Belize, Costa Rica, Cuba, Dominican Republic, El Salvador, Ecuador, Guatemala, Guyana, Honduras, Mexico, Nicaragua, Panama, Peru, Puerto Rico, Surinam and Venezuela. Nevertheless, partial sequence identification of isolates has been obtained only for Colombian, Costa Rican and Peruvian isolate and a full genome of the causal agent was just completed in 2017, for a Colombian isolate. RHBD is caused by the insect-borne tenuivirus Rice hoja blanca virus (RHBV), a multipartite single-stranded, negative sense RNA [ss(-)RNA] virus. It was first described in 1983 and partially characterized between 1992 and 1993 . The vector of RHBV is the planthopper Tagosodes orizicolus Müir (Hemiptera: Delphacidae) which are known long-distance fliers. The capacity of RHBV and related tenuiviruses to infect the insect host, explain the cyclic nature of RHBD outbreaks and its broad geographic occurrence. The recent completion of a full genome of RHBV and its comparison with related tenuiviruses found in Echinochloa and Urochloa hosts, opens the door to more detailed epidemiology and recombination studies that will improve diagnostics and the early detection of RHBV and associated tenuiviruses.

Approaches to identifying and recovering plant microbiome components contributing to plant disease suppression B. B. MCSPADDEN GARDENER, Suståne Natural Fertilizer, Inc., Cannon Falls, MN, USA

For over a hundred years, plant pathologists have pursued three distinct approaches to searching the microbiomes to find allies in their fight to suppress disease and improve plant health. The random approach recovers and, then, screens as many different microorganisms as possible to find those that most ably suppress the pathogen or disease of interest. This approach led to the formation of the first biocontrol programs and companies, and, to this day, produced the most widely sold biopesticides in the marketplace. The mechanistic approach constrains searches to one or more activities that could be assayed, ecologically, biochemically, or genetically. This second approach provided new insights into the variation and distribution of biocontrol microbes, their genes, and active metabolites. Lastly, the phylogenetic approach quantifies associations between one or more microbial components, at varying levels of organization, to identify and then recover novel microbiome components involved in disease suppression. This approach has, more recently, yielded new candidate genes, pathways, and organisms that will someday further enhance biopesticidal and biostimulatory approaches to plant health promotion. Examples of how all three approaches have been successfully implemented over the years will be used to illustrate the potential and limits of harnessing the beneficial components of microbiomes to control plant diseases in various contexts.

Balancing selection for aflatoxin in Aspergillus flavus is maintained through interference competition with, and fungivory by insects M. DROTT (1), M. G. Milgroom (1,2), (1) Cornell University, Ithaca, NY, USA; (2) Cornell University, ithaca, NY, USA

Mycotoxins contaminate large portions of world food supplies causing countless human deaths and incurring costs to growers. The role of mycotoxins in the ecology of the organisms that produce them remains poorly understood. This is even true of aflatoxin which, as one of the most potent and highly regulated mycotoxins, is also one of the most studied. Both aflatoxigenic and non-aflatoxigenic isolates of Aspergillus flavus are maintained by balancing selection, but the ecological function and impact of aflatoxin on fungal fitness is unknown. We hypothesize that balancing selection for aflatoxin production in A. flavus is driven by interaction with insects. To test this, we competed naturally occurring aflatoxigenic and non-aflatoxigenic field isolates of $A$. flavus with Drosophila larvae on medium containing 0-1750 ppb aflatoxin. We estimated fitness of $A$. flavus using qPCR to quantify its DNA. The addition of aflatoxin resulted in a 26 -fold increase in fungal fitness between 0 and $1750 \mathrm{ppb}$. With no added toxin, aflatoxigenic isolates caused higher mortality of Drosophila larvae, and had higher fitness than non-aflatoxigenic isolates. Additionally, aflatoxin production increased an average of 1.5 -fold in the presence of a single larva, and nearly 3 -fold when the fungus was mechanically damaged. Our results provide the first clear evidence of a fitness advantage conferred to A. flavus by aflatoxin when interacting with insects and have implications for future research on strategies to control aflatoxin contamination.

When pathogen populations diverge: Why understanding species boundaries is critical for managing fungicide resistance M. T. BREWER, H. Li, University of Georgia, Athens, GA, USA

An increasing number of plant diseases originally described as being caused by a single species are now known to be caused by multiple, morphologically similar, but genetically distinct species. Members of these species complexes may vary in geographic range, host specificity, fungicide sensitivity, or other factors. We recently discovered that gummy stem blight (GSB) of cucurbits is caused by three closely related, but genetically distinct species. We studied how resistance profiles are associated with two of the GSB fungi found in the southeastern U.S. Nineteen Stagonosporopsis caricae isolates and $120 \mathrm{~S}$. citrulli isolates collected from watermelon fields were genotyped with a cytb marker for azoxystrobin (QoI) resistance, and phenotyped for sensitivity to tebuconazole (DMI), boscalid (SDHI), and fluopyram (SDHI). Select isolates with varying sensitivity to DMI and SDHI fungicides were sequenced at $C y p 51$ and $S d h B$, respectively. All $S$. caricae isolates were resistant to tebuconazole and azoxystrobin, and sensitive to boscalid and fluopyram. All $S$. citrulli isolates were sensitive to tebuconazole, sensitive to fluopyram with one exception, and resistant to azoxystrobin with two exceptions. Phenotypic differences in response to boscalid were detected among $S$. citrulli isolates. We are continuing to monitor population dynamics in watermelon fields and differences in fungicide sensitivity within the two species to assess how populations may be changing in response to 
resistance profiles and fungicide use. Knowledge of population divergence and underlying biological differences, including fungicide resistance profiles, are critical for effectively managing plant-pathogenic fungi.

JA and SA signaling components are required for shade avoidance

K. Nozue (1), U. Devisetty (2), A. Bak (1), C. Casteel (1), J. MALOOF (1), (1) University of California, Davis, CA, USA; (2) University of Arizona, Tuscon, AZ, USA

Plants have sophisticated mechanisms for sensing neighbor shade and responding through enhanced elongation and physiological changes to maximize their ability to compete for light. The shade avoidance response affects many organs and growth stages, yet the signaling pathways have mostly been studied in seedlings. To understand the signaling pathways in older plants, we performed phenotypic profiling and a gene expression time course of adult shade avoidance in wild-type and shade avoidance mutants. With this data we established a signaling cascade of hormone action during wild-type response to shade and used mutants to determine how genetic perturbation affects the cascade. One finding was that $M Y C 2$, central to jasmonic acid signaling, is also required for shade induced petiole elongation. A second finding was that we found pervasive misregulation of salicylic acid genes in many mutants, suggesting salicylic acid signaling to be an important shade avoidance growth regulator. Supporting this hypothesis, several salicylic acid pathway mutants reduced shade-induced and basal growth. The effect of these mutants on shade avoidance was specific to petiole elongation, thereby defining important stage-specific differences in the downstream shade avoidance signaling pathway. Shade treatment did not change salicylic acid levels, indicating salicylic acid mediation of shade avoidance is not dependent on modulation of salicylic acid levels.

Biological and molecular characterization of citrus tatter leaf virus in Taiwan

C. Y. LIN (1), L. Chang (2), Y. H. Lin (1), M. L. Wu (3), T. H. Hung (1), (1) National Taiwan University, Taipei, TAIWAN; (2) Agricultural Biotechnology Research Center, Taipei, TAIWAN; (3) Taiwan Forestry Research Institute, Taipei, TAIWAN

Citrus tatter leaf virus (CTLV) causes chlorotic leaves with bud union incompatibility in citrus scion and its current incidence in Taiwan is $65 \%$. However, the burden of infection has long been underestimated due to its common latency, and the serious threat of tatter leaf disease should be investigated and addressed without delay. Here, the biological characteristics and genome sequences of Taiwanese isolates are evaluated. Full-length sequencing and phylogenetic analysis of three local isolates revealed similarities with isolates from neighbouring countries, indicating their possible origins. Two mild isolates obtained from Liuchen sweet orange (LCd-NA-1) and kumquat (Kq-6-2-46) were inoculated on indicator plants and exhibited differences in foliar symptom expression and temperature tolerance compared with foreign isolates causing severe disease. Improved quantitative PCRbased detection methods using redesigned primer pairs indicated high sensitivity and specificity for both local and foreign isolates. Similar but delayed systemic symptoms were observed when wildtype virus in vitro transcripts generated from a full-length infectious clone of the Ponkan mandarin isolate (pCTLV-Pk-8) were inoculated on Chenopodium quinoa. These symptoms were unevenly distributed and highly concentrated on the inoculated sides of leaves and top leaves. A single codon mutation (U5642C) in the coat protein transcription start site in the pCTLV-Pk-8 sequence resulted in defective systemic infection, indicating a role for the coat protein in viral movement. The combination of biological assays, improved detection and infectious clone construction provides comprehensive information and a foundation for citrus virus management and further research.

The role of the Global Rust Reference Center for tracking variability and spread of wheat rust fungi

M. S. Hovmøller (1), J. RODRIGUEZ ALGABA (1), T. Thach (1), M. Patpour (1), C. K. Sorensen (1), A. F. Justesen (2), S. Ali (3), P. Lassen (4), J. Grønbech Hansen (4), (1) Aarhus University, Slagelse, DENMARK; (2) Danish Inst of Agricultural Sciences, Slagelse, DENMARK; (3) University of Agriculture, Peshawar, Peshawar, PAKISTAN; (4) Aarhus University, Tjele, DENMARK

The Global Rust Reference Center (GRRC, wheatrust.org) was established in 2008 upon request from CIMMYT and ICARDA and the Borlaug Global Rust Initiative. GRRC can receive and diagnose alive samples of rust infected wheat from any country year round. So far, samples of yellow (stripe) rust (P. striiformis) and stem rust (P. graminis) from epidemic sites in Africa, Asia and Europe have main priority. GRRC is hosting the Wheat Rust Toolbox (WRT), a database driven information and communication system for global rust surveillance and pathogen genotypic and phenotypic data. Molecular genotyping is based on alive as well as dead (unrecovered) samples, whereas epidemiological important traits like virulence, aggressiveness, temperature adaptation, and in-vivo diagnosis for disease resistance, is based on alive, purified isolates. WRT is supporting BGRI institutions by web tools, maps and charts, which are integrated in several web portals, e.g. RustTracker and EuroWheat. The sharing of data and rapid release of results are in line with the European Open Science Cloud agenda reflecting the needs for timely early-warning of new epidemic outbreaks or emergence of new pathogen variants. The rapid spread of aggressive and high-temperature adapted strains of yellow rust, and emergence and spread of stem rust Ug99, and even more virulent races, have demonstrated that coordinated, international efforts are needed to mitigate the escalating threat of wheat rust epidemics worldwide.

Population Dynamics of Ectoparasitic and Endoparasitic Nematodes in North Carolina

G. GALLE, C. H. Opperman, J. P. Kerns, Department of Entomology and Plant Pathology, North Carolina State University, Raleigh, NC, USA

Sting nematode (Belonolaimus longicaudatus) and root-knot nematode (Meloidogyne spp.) are emerging problems for creeping bentgrass and bermudagrass putting greens in the transition zone. These nematodes cause severe putting green damage resulting in decreased playability. Sting nematode is a ectoparasitic nematode, feeding on the plant roots and causing severe damage at very low population numbers. Root-knot nematode is an endoparasitic nematode, feeding and reproducing within the turf root system and decreasing overall root function. However, little is known about either nematode's population dynamics and soil movement throughout the year. A sampling study was initiated in 2014 to quantify nematode populations at different depths in soil over three years. Nematode samples were collected monthly at three different depths from four golf courses throughout central North Carolina. B. longicaudatus populations were consistent across three golf courses, with numbers ranging from $40-250$ nematodes per $500 \mathrm{cc}$. soil. Populations were primarily within the top 10 centimeters of the soil during the winter, but during the summer nematodes were observed primarily in the lower $20+$ centimeters of the soil. Root-knot nematode was sampled at a single course, and numbers of stage 2 juveniles ranged from 20-120 nematodes per $500 \mathrm{cc}$. soil. Root-knot nematode populations remained in the top 10 centimeters throughout the study. Both sting and root-knot populations increased in April as temperatures start to rise. This shows very different population dynamics depending upon feeding tactics, indicating different management strategies.

Temperature-sensitive resistance breaking mechanism of Wsm 1 and $W s m 2$ genes against Wheat streak mosaic virus and Triticum mosaic virus in wheat

S. TATINENI, USDA-ARS, Lincoln, NE, USA 
Non-allelic single-dominant $W s m 1$ and $W s m 2$ genes have been deployed for management of viral diseases in wheat. Wheat cultivars Mace, carrying the Wsm1 gene, is resistant to both Wheat streak mosaic virus (WSMV) and Triticum mosaic virus (TriMV), and Snowmass, with Wsm2, is resistant only to WSMV. Wsm-based resistance in both cultivars is temperature sensitive and, in growth chamber studies, is effective at $18^{\circ} \mathrm{C}$ or below but resistance breaks at higher temperatures. The underlying temperature-sensitive resistance breaking mechanism of Wsm 1 and $W_{s m} 2$ genes in wheat cultivars was examined using fluorescent protein-tagged WSMV and TriMV. Both viruses elicited foci that were approximately similar in number and size at 18 and $24^{\circ} \mathrm{C}$ on inoculated leaves of resistant and susceptible wheat cultivars, suggesting that resistant wheat cultivars still permit cell-to-cell movement at both temperatures. Additionally, WSMV and TriMV efficiently replicated in inoculated leaves of resistant wheat cultivars at $18^{\circ} \mathrm{C}$ but failed to establish systemic infection, suggesting that Wsm 1 - and Wsm2-mediated resistance debilitated viral long-distance transport. Furthermore, neither virus was able to enter the leaf sheaths of inoculated leaves or crowns of resistant wheat cultivars at $18^{\circ} \mathrm{C}$ but both were able to do so at $24^{\circ} \mathrm{C}$. These data suggest that Wsm 1- and Wsm2-based resistance breaks at high temperatures by permitting virus entry into the vasculature for long-distance transport in resistant wheat cultivars.

A taxonomic re-examination of Ceratocystis fimbriata, the causal agent of Ceratocystis canker of almond in California L. A. HOLLAND (1), D. P. Lawrence (2), R. Travadon (2), T. Harrington (3), M. Nouri (4), F. Trouillas (5), (1) Department of Plant Pathology, University of California, Davis, Davis, CA, USA; (2) University of California, Davis, CA, USA; (3) Iowa State University, Ames, IA, USA; (4) Department of Plant Pathology, UC Kearney Ag. Research and Extension Center, Parlier, CA, USA; (5) Department of Plant Pathology, UC Davis, Parlier, CA, USA

Ceratocystis fimbriata sensu lato is an important fungal pathogen worldwide, causing various wilt and canker diseases in a wide range of hosts. In California, $C$. fimbriata infections are associated with tree wounds made by mechanical harvesting equipment and cause cankers in almond trees. The genus Ceratocystis is divided into four, distinct clades, the African, Asian, Latin American (LAC) and North American (NAC) clades. Recently, fungal isolates recovered from almond in California were classified as C. variospora in the NAC, along with isolates recovered from Quercus spp. and other woody hosts in the U.S. and abroad. However, phylogenetic analyses suggest that $C$. variospora is a paraphyletic taxon comprising multiple species. The objectives of this study were to revisit the taxonomy of Ceratocystis spp. from almond in California and to resolve cryptic species in the NAC. Multilocus sequencing of five loci ( $\beta$-tubulin, TEF1- $\alpha, 60 \mathrm{~S}, \mathrm{MCM} 7$ and Cerato-platanin genes) were used to characterize representative isolates. Results of the multi-locus phylogenetic analyses indicated several monophyletic groups of undescribed Ceratocystis species within C. variospora, including a single, novel and well-supported Ceratocystis species from California almond. This work demonstrated that there is a greater diversity of species in the NAC of C. fimbriata sensu lato than previously known.

Development outcomes and impact of scaling-up of aflatoxin biocontrol in Africa

R. BANDYOPADHYAY (1), A. Adebowale (1), M. Konlambigue (2), C. Mutegi (3), L. Senghor (4), A. Ortega-Beltran (1), P. J. Cotty (5), (1) International Institute of Tropical Agriculture, Ibadan, NIGERIA; (2) International Institute of Tropical Agriculture (IITA), Accra, GHANA; (3) International Institute of Tropical Agriculture, Nairobi, KENYA; (4) International Institute of Tropical Agriculture, Dakar, SENEGAL; (5) USDA-ARS, University of Arizona, Tucson, AZ, USA

A high proportion of maize and groundnuts produced and consumed in sub-Saharan Africa are contaminated with aflatoxin. Aflatoxin poses a significant public health risk, decreases productivity and profitability of animal industries, and hampers trade. To minimize aflatoxin contamination in maize and groundnut, a biocontrol technology, based on atoxigenic strains of Aspergillus flavus that do not produce aflatoxin, has been adapted and improved for use in Africa. Using incentivization mechanisms and commercialization approaches, this proven technology is being scaled up for use in $>500,000$ hectares in 11 African nations through a mix of public, private, and public-private-partnership interventions. Farmers and agribusinesses in Nigeria, Kenya, Senegal and The Gambia have begun treating large area of maize and groundnut fields annually and achieved significant reduction in aflatoxin contamination. Users of the technology are able to sell their aflatoxin-reduced crops to quality conscious food and feed industries at a premium thus earning higher income. At the same time, farmers also keep part of their harvest for home consumption thus improving family health. This presentation will provide evidence that large-scale deployment of biological control is improving food security, promoting trade, contributing to healthier farm families, and creating wealth.

Climate change and disease epidemiology of twig and stem blight of cotton Punjab, Pakistan S. NAZ (1), M. Iqbal (2), S. Mehboob (1), M. Ehetisham-Ul-Haq (1,2), M. Idrees (2), (1) Plant Pathology Research Institute, Ayub Agriculture, Research Institute, Faisalabad, Faisalabad, PAKISTAN; (2) Plant Pathology Research Institute, Ayub Agricultural Research Institute, Faisalabad, Faisalabad, PAKISTAN

Shifting of weather and changing intensity of environmental gradient is creating serious threats for farm economy in Pakistan. It has created the need for intensive investigations on shifting of cropping calendar epidemic and sporadic rising of diseases and threats. Cotton (Gossypium hirsutum L.) is the main cash crop of Pakistan but raises concerns over continuous drop in cotton production since last few years. While conducting a diagnostic survey in the late summer of 2015, a twig and stem blight was observed in Khanewal and Bahawalpur districts, Punjab, Pakistan at several commercial cotton production fields. Later the problem emerged as burning issue and an extensive survey was conducted in Cotton Zone of Punjab, Pakistan. These locations exhibited disease prevalence ranged 23 to $60 \%$. The morphology of the fungus was similar to Lasiodiplodia species. The pathogen expressed sensitivity against micro and meso climate of a region. The higher disease index in Khanewal and Bahawalpur districts of Punjab was due to irregular pattern of higher rainfall and early sowing. Knowledge of the disease and associated promoting factors of climate and crop management yet need experimental elaboration. The studies on climate based factors of epidemiology are in progress. However initial investigations strengthen the involvement of irrigation technique, soil type and cropping history in introduction and sporadic occurrence of the disease.

\section{Progress on chemical management of postharvest diseases of subtropical fruits}

J. ADASKAVEG (1), H. Forster (2), D. Chen (1), (1) Department of Plant Pathology and Microbiology, University of California, Riverside, CA, USA; (2) Department of Microbiology and Plant Pathology, University of California, Riverside, CA, USA

Application of postharvest fungicides is a highly effective, targeted, and economical way to protect fruit from decay after harvest and during shipment and marketing, while fungicide residues remain within MRLs, similar to preharvest field applications. These treatments are especially beneficial for fruits that are stored for extended times or that are shipped long distances. The United States has been on the forefront in developing these treatments. For sub-tropical crops such as citrus, pomegranates, pineapples, and avocados, new fungicides have been introduced to manage major diseases. Fungicides range from conventional (propiconazole) to reduced-risk (azoxystrobin, fludioxonil) to biopesticide (natamycin) compounds, and also include pre-mixtures (fludioxonil-propiconazole, -azoxystrobin, or -cyprodinil). Azoxystrobin-fludioxonil is effective against decays caused by DMI ${ }^{\mathrm{SR}}$ and $\mathrm{MBC}^{\mathrm{SR}}$ isolates of Penicillium spp.; propiconazole is effective against Geotrichum and DMI ${ }^{\mathrm{s}}$ and $\mathrm{MBC}^{\mathrm{SR}}$ Penicillium spp. Natamycin has activity against 
Geotrichum and Penicillium (DMI ${ }^{\mathrm{SR}}$ and $\mathrm{MBC}^{\mathrm{SR}}$ ) decays, is exempt from tolerance in North America, and potentially is an organic treatment. Although moderately effective by itself, mixtures provide high efficacy and resistance management. Fludioxonil-propiconazole, -azoxystrobin, and -cyprodinil premixtures, as well as natamycin are effective for managing Alternaria, Botrytis, Ceratocystis, Colletotrichum, Fusarium, Rhizopus spp., and other molds.

Bioformulation of Trichoderma harzianum for the management of soil borne plant diseases P. DUTTA, Assam Agricultural University, Jorhat, INDIA

Potential native strains of Trichoderma harzianum were isolated from vegetable and tea growing areas of Assam and West Bengal, India. In vitro and in vivo assay showed effective result against six (6) soil borne fungal plant pathogens viz., Rhizoctonia solani, Sclerotinia sclerotiorum, Sclerotium rolfsii, Fusarium oxysporum, Colletotrichum capsici as well as root knot nematode viz., Meloidogyne incognita causing diseases in vegetables, tea and turmeric Considering the interest of farming community, protocol for liquid bioformulation (Org-Trichojal) of the potential strain of $T$. harzianum was standardized with a shelf life of 480 days. Further in vitro and field study showed its potentiality against the above pathogens and also found to act as plant growth promoter. Farmers, extension personnel's and tea garden managers etc were trained on technical aspects of the bioformulations and its field use. The product Org-Trichojal is now gaining popularlity among the farmers, corporate sectors of Assam, India.

Quads experience towards international harmonisation of methods development and validation procedures for regulatory purposes B. Rodoni (1), L. Levy (2), P. J. Shiel (3), M. K. NAKHLA (4), (1) Plant Biosecurity Cooperative Research Centre, Bruce, AUSTRALIA; (2) USDA APHIS PPQ CPHST, Riverdale, MD, USA; (3) USDA APHIS PPQ S\&T CPHST, Raleigh, NC, USA; (4) USDA-APHIS-PPQ-S\&T-CPHST, Beltsville, MD, USA

The Quadrilateral Scientific Collaboration in Plant Biosecurity (Quads) Molecular Diagnostics Working Group is part of a scientific collaboration in plant biosecurity between the Australia, Canada, New Zealand and USA focused on the development of diagnostic protocols, and associated laboratory requirements and processes. This Collaboration provides a framework for scientific cooperation regarding plant protection, including: phytosanitary issues; identifying and sharing diagnostic tools and technologies; quality assurance and control methods; and developing common language and best practices not covered by other organisations. To facilitate harmonisation of diagnostic standards the working group has generated guidelines for method acceptance, a compendium of diagnostic methods from the four participating countries, and a guidance paper on proficiency testing. These items developed and shared between the countries highlight the importance of Quads activities. The application of Next Generation Sequencing (NGS) as a diagnostic tool is identified as both an opportunity and a challenge for diagnostic laboratories. A framework for the application of NGS as a diagnostic tool has been generated by the working group. It has been recognized that the absence of guidelines for the interpretation of NGS data sets by researchers, diagnosticians and regulators required to inform policy makers could adversely impact biosecurity. Activities in this space at a global level will be discussed.

Blackleg in South African Potato production: Pathogens and impact

J. VAN DER WAALS, University of Pretoria, Pretoria, SOUTH AFRICA

The sustainable production of potatoes in South Africa is hampered by many biotic and abiotic constraints. Among the most important biotic factors is the disease complex caused by the Soft Rotting Pectobacteria (SRPs). The symptoms caused by these bacteria include poor emergence, blackleg, wilt, aerial stem rot and soft rot of tubers post-harvest. Pathogens causing this disease in South Africa include Pectobacterium carotovorum subsp. brasiliense (Pcb), P. carotovorum subsp. carotovorum (vPcc) and to a lesser extent $P$. parmentieri and Dickeya dadantii (Dd). The most important and widely spread causal agent of this disease is Pcb. This bacterium has resulted in significant losses in field, primarily due to pre-emergence soft rotting of seed, leading to poor stands. In the period between 2006 and 2010, Pcb made up 77\% of the SRE population isolated from potatoes. This percentage decreased between 2011 and 2013, to about 40\%. The percentage of virulent vPcc strains increased from about 10\% of the SRP population between 2006 and 2010 , to $35 \%$ in the period from $2011-2013$. This population shift from dominance of primarily Pcb to both Pcb and vPcc coincided with a decrease in blackleg incidences in seed potato fields from the early 2000s to after 2010. Currently, Pcb is still the most important blackleg-causing pathogen in South Africa. Dd is the only reported member of this genus in South Africa and constitutes a small proportion of the blackleg-causing pathogens in the country. Despite the decrease in blackleg in seed plantings, the prevalence of the disease in ware potato plantings has continued to increase, resulting in financial losses to growers. Warm temperatures during the planting seasons, irrigation, countrywide transport of seed, contaminated irrigation water and planting of susceptible cultivars could have contributed to this steady increase in disease.

Yield loss to Fusarium pseudograminearum of commercially grown barley and wheat varieties in Western Australia D. HUBERLI, M. Connor, K. Gajda, Department of Primary Industries and Regional Development, South Perth, AUSTRALIA

Several new wheat varieties claim to have improved tolerance to Fusarium crown rot, one of the major soil borne disease constraints on wheat and barley production in the low rainfall environments of Western Australia (WA). This improvement is particularly important for cereal growers from low rainfall environments where choice of alternative crop species are limited and tight cereal rotations are common. Testing crown rot tolerance of commercially grown wheat and barley varieties will allow growers to evaluate the economic benefits of varietal selection in fields with high crown rot inoculum. A meta-analysis will be presented of a three year series (2014-2016) of experiments in two locations with 12 barley and wheat varieties in paired plot comparisons with and without Fusarium pseudograminearum inoculum. Although all barley and wheat varieties were impacted by crown rot symptoms, the level of yield impact varied between varieties. Emu Rock, a variety released as having 'improved tolerance' did prove to be consistently less affected by crown rot than other commercial varieties. For example yield losses in Emu Rock ranged from 0-22\% while Justica CL Plus consistently suffered the largest yield reduction, ranging from $23-42 \%$. Differences in crown rot tolerance had not previously been characterised for commercial barley varieties but differences were evident from these experiments. Litmus and La Trobe had consistently lower yield loss than most other barley varieties in the presence of crown rot, while Bass and GrangeR were consistently most affected. Understanding the crown rot disease history of a field and choosing wheat or barley varieties with appropriate disease tolerances can improve stability of crop yields substantially.

Rust epidemics: Are the alternate hosts the culprit?

A. BERLIN (1), B. Andersson (1), J. Yuen (2), B. Samils (2), J. Oliva (3), (1) Swedish Univ of Agricultural Sciences, Uppsala, SWEDEN; (2) Swedish University of Agricultural Sciences, Uppsala, SWEDEN; (3) Dept of Forest Pathology, Lleida, SPAIN

Most plants can be infected by rust fungi. The rust fungi may have up to five spore stages and many have two hosts, often one perennial and one annual plant species. The sexual cycle is completed on the aecial hosts, which creates new variants of the fungi through genetic recombination. In addition, each 
aecium or aecial cup may be a unique individual and only share half of its genetic material with its sibling in the neighboring cup. This creates a large amount of genetic diversity from each successful completion of the rust fungal life cycle. Concurrently, the asexual, clonal propagation on the telial host will enable successful fungal individuals to produce enormous amounts of spores of the same genotype. To investigate the role of the alternate host, the population biology of different types of rust species have been studied. If a rust fungal population completes its sexual stage within the region, compared to if it survives in its clonal, uredinial stage, it will have a profound effect on the epidemiology of the disease. A question is what the advantage is for rust fungi to have two hosts. By using two seasons samples from a spore trapping network, and molecular detection of rust fungi through sequencing, a factor describing the quantity of different spores stages could be inferred by calculating the relative abundance of the number of sequence reads, in connection with data on the phenology of the hosts. To understand the contribution of each spore stage in an epidemic, these factors were included in a model to describe different epidemiological scenarios.

The first seven days: Spatiotemporal transcriptome analysis of infection of a wheat flower by the ergot pathogen Claviceps purpurea A. GORDON (1), E. Tente (2), N. Ereful (1), P. Grant (3), D. O'Sullivan (4), L. Boyd (5), (1) NIAB, Cambridge, UNITED KINGDOM; (2) NIAB / University of Cambridge, Cambridge, UNITED KINGDOM; (3) Microsoft Research, Cambridge, UNITED KINGDOM; (4) School of Agriculture, Policy and Development University of Reading, READING, UNITED KINGDOM; (5) NIAB - The Bingham Laboratory, Cambridge, UNITED KINGDOM

Claviceps purpurea, the causal fungus of ergot, poses a threat to human and animal health through the hazardous ingestion of ergot alkaloids. $C$. purpurea infects cereals via the receptive stigma hairs of a developing flower, with the hyphae then colonising the ovary tissue and producing an overwintering fungal structure, known as an ergot sclerotia. In this study we analysed the interaction between a C. purpurea isolate from the UK and a male sterile hexaploid wheat line, assessing gene transcription across eight timepoints over the crucial first week of infection. We precisely dissected wheat flowers, using confocal microscopy to image fungal development and constructed an interactive biological map tracking the infection of host tissue, and the corresponding expansion and proliferation of the pathogen. Here, we relate this biological time course to transcriptional changes in the host in defence, photosynthesis and plant hormone pathways, and to coupled changes in pathogen development and alkaloid biosynthesis.

Quantifying the Spatial and Temporal Variations of Plant-Parasitic Nematodes Associated with Corn in Ohio

A. C. M. SIMON (1), R. Lewandowski (2), E. Richer (3), T. L. Niblack (4), P. A. Paul (5), (1) The Ohio State University, Columbus, OH, USA; (2) Ohio Sate University Extension, Athens, OH, USA; (3) Ohio State University, Wauseon, OH, USA; (4) The Ohio State University, Columbus, OH, USA; (5) The Ohio State University, Wooster, OH, USA

The vertical distribution of five plant-parasitic nematodes was examined in two Ohio corn fields, one with a silt loam soil in Wayne county and another with sandy loam in Fulton county. Population densities of Pratylenchus crenatus, P. thornei, Helicotylenchus pseudorobustus, Paratylenchus neoamblycephalus, and Tylenchorhynchus claytoni were determined from soil samples taken during corn growth stages VE to V10 at $10 \mathrm{~cm}$ increments to a depth of $70 \mathrm{~cm}$. $P$. crenatus and $P$. thornei predominantly inhabited the top $40 \mathrm{~cm}$ of soil and occurred at significantly higher densities between growth stages V6 to V10, particularly in the root fraction. The highest population densities of $H$. pseudorobustus were found in the upper $20 \mathrm{~cm}$ of the soil profile at the Fulton location, and predominantly in the top $40 \mathrm{~cm}$ at the Wayne location at all growth stages monitored. P. neoamblycephalus was also detected at both locations, with the highest population densities at the $20 \mathrm{~cm}$ depth in Wayne, regardless of growth stage, and at the $40-60 \mathrm{~cm}$ depth in Fulton at the V3-V4 growth stages. T. claytoni was recovered from the Fulton field only, predominantly inhabiting the top $40 \mathrm{~cm}$ of the soil profile where counts were generally highest between growth stages V6 to V7. Our findings suggest that the depth and growth stage at which samples are collected to estimate nematode population density should vary with the nematode species in question.

Collaborative approaches in USAID global IPM to implement practical solutions to virus diseases by detection, diagnosis, and capacity building S. A. TOLIN, Virginia Polytechnic Institute and State University, Blacksburg, VA, USA

Over two decades ago the U.S. Agency for International Development (USAID) began funding a participatory IPM project operating in four global regions with teams of U. S. scientists from entomology, phytopathology, economics, and gender studies. Toward the end of the first 5-year phase, participatory appraisals in Jamaica recognized a disease caused by a virus in the targeted non-traditional export crop, Scotch bonnet pepper. The crop's export value had increased production acreage, demanded higher quality, and intensified scaleup of seed and seedling production. The local scientists asked the USAID project to add virologists to assist in research on the virus/vector/host pathosystem, which led to a validated IPM approach. In later phases, the expertise in virology grew as a funded 'global theme' provided research collaborators on virus problems across several crops and regions. One focus was on diagnosis and data compilation of viruses in various vegetable crops in up to 19 countries. Vector identity, dynamics, and control were factored into implementing IPM packages. However, tropical regions with consecutive cropping and continuous vegetation posed challenges to tactics of vector and virus reservoir management often used in temperate climates. Close monitoring of systems for producing seeds and seedlings suggested virus introduction at these steps, requiring other monitoring tactics, and confirming there is no "one-size-fits-all" approach. In field and laboratory diagnostic workshops, host country scientists and students were trained to recognize symptoms associated with virus infections and to develop the capacity to conduct high-throughput, low cost detection and diagnostic procedures as independent researchers.

An interspecific barberry hybrid enables genetic dissection of non-host resistance to the wheat stem rust pathogen R. BARTAUlA (1), A. Melo (1), S. Kingan (2), I. L. Hale (1), (1) University of New Hampshire, Durham, NH, USA; (2) Pacific Biosciences, Menlo Park, CA, USA

Stem rust, caused by Puccinia graminis $(P g)$, is one of the most destructive diseases of wheat worldwide; and the threat of $P g$ to global food security is enhanced by its ability to rapidly evolve new forms of virulence. The emergence of the highly virulent wheat stem rust races, most notably the $U g 99$ family of stem rust races radiating out of East Africa, underscores the need for durable mechanisms of genetic resistance to $P g$. Due to its intrinsic durability, non-host resistance (NHR) presents a compelling vision; but NHR to Pg remains largely unexplored as a protection strategy in wheat, in part due to the challenge of developing a genetically tractable system in which $P g$-NHR segregates. In this study, we approached the question of $P g$-NHR through the pathogen's sexual host, barberry (Berberis spp.). Using an interspecific mapping population derived from a cross between $P g$-susceptible $B$. vulgaris and Pg-resistant $B$. thunbergii, we have begun to dissect the genetics of the Pg-NHR exhibited by $B$. thunbergii. Building on our $1.3 \mathrm{~Gb}$ genome for $B$. thunbergii and genetic linkage maps for both parental species, QTL analysis identified a single QTL for $P g$-NHR, spanning $\sim 7 \mathrm{cM}$ ( $\sim 1.74 \mathrm{Mbp}$; $\mathrm{LOD}=33.16)$. Candidate genes are prioritized for follow-up study based on both differential expression under $P g$ challenge and function-related $B v-B t$ sequence variation. With this study, we demonstrate for the first time the feasibility of dissecting the genetics of a source of $P g$-NHR, which may contribute insight into possible novel mechanisms of durable rust resistance in wheat. 
During activated states of immunity plants often display a tradeoff between defense and growth. High levels of defense activation leads to reduced shoot growth and also reduced seed set. This phenomenon is observed in Arabidopsis, and many other plant species, however the mechanisms behind growth defense tradeoffs are mostly unknown. Hormone signaling networks are responsible for regulating growth and development, as well as plant-pathogen interactions. To identify possible molecular mechanisms modulating the growth defense trade-off in plants we focused on the plant growth hormone cytokinin, widely known for a role in plant growth and development and for which a role in defense responses to biotrophic pathogens has been recently demonstrated. We used a computational biology approach to analyze transcriptional data for regulators of plant growth-defense tradeoffs. With this approach we identified a class of transcription factors that are important for cytokinin-promoted plant growth, and whose expression is rapidly reduced during defense activation. Genetic analyses showed that mutations in these genes leads to increased resistance to bacterial and oomycete pathogens, as well as increased defense gene expression. Overexpression of these genes positively affects plant growth. We propose that these transcription factors may function as a switch between growth and defense, through the regulation of cytokinin-regulated transcriptional targets that prioritize plant growth over defense activation.

Open Science and International Collaboration to Tackle the Fearsome Wheat Blast in Asia and Beyond M. T. Islam (1), S. KAMOUN (2), (1) Bangabandhu Sheikh Mujibur Rahman Agricultural University, Gazipur, BANGLADESH; (2) The Sainsbury Laboratory, Norwich, UNITED KINGDOM

Wheat blast, caused by Magnaporthe oryzae Triticum pathotype (MoT), is one of the emerging threats to wheat production worldwide. It was first emerged in Parana state of Brazil in 1985 through host jump from a local grass and then spread to several South American countries. In February 2016, the first outbreak of wheat blast in Bangladesh devastated more than 15,000 hectares of wheat. In a rapid response to a new threat of food security of Bangladesh, we (31 researchers from 4 continents) applied field pathogenomics and open data sharing approaches and determined the genetic identity and origin of the disease within weeks. In phylogenomics analyses, the Bangladeshi MoT isolate was shown to be closely related to the highly aggressive wheat-infecting South American MoT strain, suggesting that the wheat blast fungus was most likely introduced from South America. This year wheat blast was detected in the new areas of Bangladesh and also in West Bengal of India. Genetic resources for the resistance breeding are limited and fungicide application seem unreliable. A larger scale international collaboration and application of novel approaches are needed to mitigate this enemy of food security. To share our data with global scientific community, we uploaded the whole genome sequences of a large number of wheat blast fungal strains in open wheat blast website (http://wheatblast.net), which were isolated from the infected field of Bangladesh. Whole genome sequences of four potential wheat blast biocontrol bacteria were also uploaded. We are currently working on genome editing of the $S$-genes in wheat by using CRISPR/Cas9 technique to develop new blast resistant wheat varieties for Bangladesh. We are dedicated to share our data through the said website and urge scientific community to work together for combatting this enemy of wheat before it becomes catastrophic in Asia.

On the mechanism of translocation of Magnaporthe oryzae effectors into rice cells E. OLIVEIRA GARCIA (1), M. Martin-Urdiroz (2), C. Rodriguez Herrero (2), N. Talbot (2), B. Valent (1), (1) Kansas State University, Manhattan, KS, USA; (2) Univ of Exeter, Exeter, UNITED KINGDOM

Rice blast caused by Magnaporthe oryzae is the most destructive disease of rice worldwide. M. oryzae secretes various effectors, which are hypothesized to be involved in effective host infection. To date, little is known about the mechanisms of effector uptake into plant cells. Here we show translocation of the cytoplasmic effectors Bas1, Pwl1 and Pwl2 in vesicles from the biotrophic interfacial complex (BIC) to rice cytoplasm during biotrophic development. Using fluorescent protein tagging, we found that cytoplasmic effectors Bas1, Pwl1 and Pwl2 are sorted into different vesicles in BICs formed on primary hyphae, revealing new levels of functional complexity for this biotrophic structure. Additionally, the effector Bas 83 is plant plasma membrane- and BIC-localized, suggesting a potential role in vesicle formation. Through FM4-64 labeling and Lit6b:eGFP transgenic rice colocalization assays, we demonstrated that the vesicles containing effectors originate from plant plasma membrane. Furthermore, plant endocytosis inhibitors and Virus-Induced Gene Silencing of rice endocytosis components induce abnormally-shaped and swollen BICs, accumulation of cytoplasmic effectors in $\mathrm{BICs}$, as well as the accumulation of the cytoplasmic effectors under penetration pores. Based on these results we conclude that cytoplasmic effector translocation is mediated by vesicle formation and may be characteristic of appressoria as well as biotrophic invasive hyphae.

\section{Plant Disease Extension in Mexico}

H. LOZOYA-SALDANA, Univ Auto Chapingo, Chapingo Mexico, MEXICO

Mexico is located in a tropical-subtropical region (14-32 ${ }^{\circ} \mathrm{N}$ latitude), with agricultural regions throughout the country, mostly rain-fed ( $74 \%$ of the area), with mild winters and year-round exposure to plant diseases. Agricultural extension in Mexico was formally created in the early 1950's, copying the US "Land Grant" model that lasted as such for about 30 years. Nowadays, the lack of a formal Federal Extension Service regarding plant health is made up for by State Committees, coordinated by the Federal Plant Health Agency. It includes a number of officers strategically posted in every State according to the intensity of the agricultural activities, through Programs or working plans, one each per State, and 20 current "Campaigns", based on the economic importance of the crop and/or the pest (insects, weeds, diseases), like coffee rust and the coffee borer beetle, citrus viruses and Huanglongbing, avocados, cotton, sorghum, bananas, agaves, etc. In addition, this service is also offered at most of the 38 INIFAP Experiment Stations (equivalent to the USDAARS), with seven general extension models. Also, more than 50 local universities provide assistance to farmers, and most of the plant pathologists of these institutions comprise the Mexican Society of Phytopathology, founded in 1967, with about 300 active members. Alternative extension models are continuously proposed according to regional socio-economic particularities.

Investigating the diversity and function of secreted Clavibacter effectors

S. Thapa (1), Q. Lyu (1), S. Pattathil (2), M. G. Hahn (2), R. L. Gilbertson (3), G. L. COAKER (1), (1) University of California, Davis, CA, USA; (2) University of Georgia, Athens, GA, USA; (3) Department of Plant Pathology, University of California, Davis, CA, USA

Clavibacter michiganensis subsp. michiganensis $(\mathrm{Cmm})$ is a Gram-positive bacterial pathogen that proliferates in tomato xylem vessels causing bacterial canker disease. Bacterial canker can cause substantial losses in greenhouse and field production systems. Investigations of the molecular basis of pathogenicity have been primarily performed on the reference strain Cmm NCPPB832. In this study, we used a combination of short and long read sequencing technologies generate high quality genome sequences for $11 \mathrm{Cmm}$ strains isolated from field-grown tomatoes in California. We performed a comparative genomic analyses to identify conserved secreted type II effector proteins. The largest classes of conserved secreted $\mathrm{Cmm}$ effectors were serine proteases and carbohydrate-active enzymes (CAZymes). Glycome profiling revealed that $\mathrm{Cmm}$ strains were able to extensively and specifically 
alter tomato cell-wall composition, likely through CAZyme activity. The conservation and function of specific conserved $\mathrm{Cmm}$ effectors will be reported. Collectively, these data provide a more comprehensive understanding of $\mathrm{Cmm}$ diversity and virulence strategies.

Effects of UV photocatalytic ethylene removal on interactions between Colletotrichum gloeosporioides and Solanum lycopersicum (tomato) fruit A. FLETCHER, M. J. Dickinson, University of Nottingham, Loughborough, UNITED KINGDOM

Anthracnose, caused by Colletotrichum gloeosporioides, creates major problems in the postharvest market; particularly in tropical fruits. The pathogen causes significant economic loss due to its ability to remain asymptomatic at harvest before switching to a necrotrophic, aggressive stage of infection. A treatment that prevents disease in storage and transport environments would significantly reduce the amount of waste caused by the fungus. Tomato fruits were used as a model and inoculated with C. gloeosporioides prior to storage in either a chamber fitted with a UV photocatalytic ethylene removal system, or a control chamber. Fruits were incubated at $16^{\circ} \mathrm{C}$ for 4 weeks. Constant ethylene removal was successful in reducing the occurrence and severity of anthracnose without significantly effecting the fruits physiological appearance. Control chamber fruits were decimated by severe disease, whereas fruits in the ethylene removal chamber remained healthy and symptomless. Ongoing research is focused on elucidating the molecular mechanisms induced by ethylene removal in both pathogen and fruit to determine how this treatment is preventing disease. These findings could have a significant impact on the postharvest market since the UV photocatalytic ethylene removal system is easy to install and implement in all steps of the supply chain. Prevention of anthracnose observed due to this treatment could result in major reductions in fruit losses caused by C. gloeosporioides.

Cross-kingdom small RNA trafficking and environmental RNAi for plant protection against Fungal pathogens H. JIN, Department of Microbiology and Plant Pathology, University of California, Riverside, CA, USA

Small RNAs (sRNAs) play a critical role in both host innate immunity and pathogen virulence. We have demonstrated that some sRNAs from eukaryotic pathogens, such as Botrytis cinerea, the fungal pathogen that causes grey mold disease on more than 1000 plant species, are translocated into host plant cells and suppress host immunity genes for successful infection. These sRNAs act as a novel class of pathogen effectors that translocate into host cells to suppress host immunity. Conversely, we also found that some plant-derived sRNAs, including both exogenous and endogenous sRNAs, are transferred into interacting fungal cells. Transgenic plants expressing hairpin RNAs that targeting Botrytis Dicer 1 and Dicer 2 genes could effectively silence fungal DCL genes, and inhibit the generation of fungal sRNA effectors to suppress grey mold disease. This strategy can be easily designed to control multiple fungal diseases simultaneously. We also identified a set of host endogenous sRNAs that are delivered into fungal cells, mainly through extracellular vesicles, to suppress fungal genes that are involved in pathogenicity. Strikingly, we discovered that some fungal pathogens, such as Botrytis cinerea and Sclerotinia sclerotiorum. could take up double-stranded RNAs and sRNAs from the environment. Applying sRNAs or dsRNAs that target fungal Dicers and pathogenicity-related genes on the surface of fruits, vegetables and flowers significantly inhibits fungal diseases. Such pathogen gene-targeting RNAs represent a new generation of effective and eco-friendly fungicides.

Polymer nanoparticles as potent fungicides against Verticillium dahliae: Insights from a metabolomics perspective M. LYKOGIANNI (1), Z. Sideratou (2), D. Tsiourvas (2), K. Aliferis (1,3), (1) Laboratory of Pesticide Science, Agricultural University of Athens, Athens, GREECE; (2) Institute of Nanoscience and Nanotechnology, NCSR Demokritos, Athens, GREECE; (3) McGill University, Montréal, CANADA

The lack of efficient plant protection products (PPPs) against devastating plant diseases is among the major challenges that the agri-food sector is facing. Taking into account the slow introduction of molecules exhibiting new mode(s)-of-action (MoA), the recent developments in nanotechnology could provide a valuable alternative towards improving crop protection. Nanoparticles could be used as novel active ingredients per se or nanocarriers in the nanoformulation of the next generation (nano)-PPPs. Nonetheless, there is an increasing demand for the in-depth study of their bioactivity and fate in the environment. Within this context, the application of metabolomics, could greatly assist towards understanding the bioactivity and MoA of nanoparticles. Therefore, we have undertaken the task of mining the effects of hyperbranched poly(ethyleneimine) nanoparticles on the metabolism of Verticillium dahliae metabolism applying metabolomics, which is a very important plant pathogen, in an effort to gain insights into their MoA. Analyses revealed a variable impact of the applied nanoparticles on $V$. dahliae metabolism, which is indicative of the operation of distinct MoA. Several biosynthetic pathways involved in fungal responses to stresses were up-regulated, clearly indicating the toxic effect of the applied nanoparticles. Results highlighted the potential of metabolomics in the study of the MoA and toxicity of polymer nanoparticles and that of the latter as new PPPs.

Development of cisgenic lines of apple to enhance resistance to fire blight

G. A. L. BROGGINI (1,2), I. Schlathölter (1,2), B. Studer (3), A. Patocchi (2), (1) Molecular Plant Breeding, Institute of Agricultural Sciences, ETHZ, Wädenswil, SWITZERLAND; (2) Breeding Research, Agroscope, Wädenswil, SWITZERLAND; (3) Molecular Plant Breeding, Institute of Agricultural Sciences, ETHZ, Zurich, SWITZERLAND

Almost all commercially successful apple cultivars are susceptible to fire blight, a devastating disease caused by the bacterium Erwinia amylovora. To control the disease in apple orchards, several approaches were attempted, including large-scale eradication programs and application of antibiotics or biocontrol agents. In addition, apple genotypes showing natural resistance to the disease were identified and allowed the study of the genetic architecture underlying the resistance mechanism. Breeding for fire blight resistant cultivars of commercial value was initiated, but this process is hampered by the long juvenility and the allogamy of apple. With each cross the traits of a successful parent cultivar are recombined. The breeder must identify a superior combination of favorable traits, leading to a new cultivar, amidst a large number of seedlings. The investigation of the Malus $\mathrm{x}$ robusta 5 resistance led to the cloning of the fire blight resistance gene $F B \_M R 5$, encoding for an NBS-LRR protein. FB_MR5 was used to generate C44.4.146, a fire blight resistant cisgenic line of the cultivar 'Gala Galaxy'. The cisgenic approach, i.e. the genetic modification resulting in a genotype carrying exclusively genes from a sexually compatible donor, should retain the original cultivar characteristics adding the desired resistance. For three years, the phenotype of C44.4.146 has been investigated in a field trial at the "Protected Site". This is a three hectares plot dedicated to field experiments with genetically modified plants located in Zurich, Switzerland. First results of the phenotypic and the molecular characterization of the fire blight resistant cisgenic line C44.4.146 will be presented.

Concepts and parameters for modeling the persistence of human enteric pathogens on plants and related foodborne epidemics M. T. BRANDL, Produce Safety and Microbiology Research Unit, USDA/ARS, Albany, CA, USA

The occurrence of foodborne disease linked to contaminated fruit and vegetables has become an important concern in crop management and public health. Several field studies have demonstrated that human enteric pathogens survive, albeit generally poorly, after their inoculation onto plants, but few clues have emerged regarding the factors that allow for this persistence and its relation to human disease. In order to further our understanding of the ecology of human pathogens on plants and better predict the probability of an outbreak resulting from a contamination event, the heterogeneous nature of 
plant surfaces at various scales must be considered. Ecological concepts and parameters that may inform a model of the behavior of bacterial foodborne pathogens, such as Salmonella enterica and Shiga toxin-producing E. coli, at a range of scales in a healthy and compromised plant habitat and in the broader plant environment will be discussed.

Diversity and distribution of Phytophthora species in Protected Natural Areas of Sicily, southern Italy

F. LA SPADA (1), F. Aloi (1,2), A. Pane (1), S. O. Cacciola PhD (1), (1) Department of Agriculture, Food and Environment, University of Catania, Catania, ITALY; (2) Department of Agriculture and Forestry Sciences, University of Palermo, Palermo, ITALY

Sicily, southern Italy, is located in the centre of the Mediterranean basin, one of the most important biodiversity hotspot areas. Numerous Protected Natural Areas (PNAs) have been established in the island for preserving its ecological integrity. The aim of this study was to investigate the occurrence and diversity of Phytophthora species in PNAs of Sicily and to explore if, and to which extent, diversity correlates with vegetation type(s). Twenty-nine natural ecosystems (15 forest stands and 14 riparian systems) from 12 PNAs were surveyed. Phytophthora species were isolated by baiting and isolates were identified by combining macro- and micro-morphological features with phylogenetic inference (ITS-rDNA sequence analysis). Overall, 18 Phytophthora species from eight of the 12 known phylogenetic clades were recovered. These included the two newly described $P$. tyrrhenica and $P$. vulcanica, and 11 among the most aggressive introduced species, including $P$. $\times$ cambivora, $P$. cactorum, $P$. citrophthora, $P$. crassamura, $P$. hydropathica, $P$. multivora, P. plurivora, $P$. polonica, $P$. pseudocryptogea, $P$. thermophila and $P$. sp. kelmania. The distribution of $P$ hytophthora species in different ecosystems and its ecological implications are discussed.

Impacts of antibody and molecular disease diagnostics kits on disease identification and management in Africa S. A. MILLER, Department of Plant Pathology, The Ohio State University, Wooster, OH, USA

Accurate diagnosis is the essential first component of plant disease management, and misdiagnosis can lead to control failures, economic losses and the health and environmental consequences of pesticide misuse. The accuracy of symptom-based diagnostics is a function of the knowledge and experience of the practitioner or grower, but symptoms alone are often inadequate. Molecular and serological assays serve as confirmatory backups to symptombased diagnostics, and essential tools in the identification and surveillance of emerging and invasive pathogens. While the relatively high cost of on-site serological and isothermal nucleic acid amplification assays generally preclude their routine use in Africa, the use of laboratory-based assays for pathogens such as Ralstonia solanacearum, Xanthomonas campestris pv. musacearum and the viruses causing maize lethal necrosis have allowed local researchers to identify pathogens, monitor their emergence across regions and improve plant safeguarding capability. Access to diagnostics assays has also led to local development of management tactics based on knowledge of pathogen etiology and epidemiology. Laboratory infrastructure and personnel training in serological and molecular diagnostics have improved significantly in the past decade but further investments in capacity building are needed to promote sustainable disease management and safeguard crops throughout the continent.

To be host or not to be: The role of asymptomatic hosts in the management of Verticillium wilt of potato L. BAUTISTA-JALON (1), M. G. Milgroom (2), B. K. Gugino (1), O. Frenkel (3), L. L. Tsror (4), M. D. M. Jimenez-Gasco (1), (1) The Pennsylvania State University, University Park, PA, USA; (2) Cornell University, Ithaca, NY, USA; (3) Volcani Center, ARO, Rishon Lezion, ISRAEL; (4) Volcani Center, ARO, MP Negev, ISRAEL

Sustainable management of Verticillium wilt of potato is challenged by Verticillium dahliae $(\mathrm{Vd})$ broad host range and long-term persistence in soil, and the lack of host resistance. Crop rotations are essential for disease management but they do not always render the expected results due to the ability of $V d$ to infect weeds and rotational crops as an endophyte. Our goal was to assess the genetic structure of $V d$ populations infecting symptomatic and asymptomatic host plants recovered from potato fields affected by Verticillium wilt in the US and Israel. Our hypothesis was that populations that infect hosts as endophytes may represent lineages that are genetically distinct from those pathogenic to potato. A phylogeny inferred from 26,934 SNPs among $144 \mathrm{Vd}$ isolates displayed a highly clonal structure correlated with VCGs, and isolates grouped in lineages $2 \mathrm{~A}, 2 \mathrm{~B}^{824}, 4 \mathrm{~A}$ and $4 \mathrm{~B}$. Lineage $4 \mathrm{~A}$, highly virulent to potato, was found only among US isolates from symptomatic potato. Isolates from asymptomatic hosts grouped mainly within lineage 4B and were not genetically differentiated by host. Phylogeny of $4 \mathrm{~B}$ revealed a higher diversity in the US compared to Israel and coalescent analysis from 174 SNPs showed a divergent genealogy based on geographic origin indicating a single introduction of this lineage in Israel. We conclude that asymptomatic secondary hosts can serve as reservoirs to maintain $V d$ populations of lineage $4 \mathrm{~B}$, which are pathogenic to many cultivated hosts.

Seed health challenges in the smallholder informal seed system

Q. KRITZINGER (1), T. A. S. Aveling (2), (1) Department of Plant and Soil Sciences, University of Pretoria, Pretoria, Gauteng, SOUTH AFRICA; (2) Department of Plant and Soil Sciences, FABI, University of Pretoria, Pretoria, Gauteng, SOUTH AFRICA

Seeds play an important role in the livelihoods of smallholder farmers in many countries, especially in Africa. The freshly harvested seed and/or on-farm stored seed are sold at informal markets for generation of income. Smallholder farmers usually cannot buy good quality certified seed for planting and rely on their own on-farm-saved seed or that obtained from other smallholder farmers for the next season's production. The transfer of poor quality and possibly infected seed is largely uncontrolled. This seed often has reduced germination and seedling vigour, and is vulnerable to fungi, in both storage and the field, which may produce mycotoxins causing serious health complications in both animals and humans. Farmers use different containers (e.g. metal tanks, re-used maize meal sacks) and techniques (e.g. sun drying, seed mixed with ash) to store their seeds. However, fungal infestation of seed due to high temperatures and relative humidity can occur. Effective control and prevention strategies at the level of the smallholder farmer are required, which can be achieved by optimizing storage systems and assistance from trained extension officers. The seed health challenges existing within the smallholder seed system will be reviewed, with emphasis on the challenges faced during postharvest and storage. The negative impact of seed movement across borders due to poor implementation of regulations will also be discussed.

A fungal invasion is enhanced by hybridization and gene introgression: Ecological and evolutionary implications of genomic admixing P. GONTHIER (1), F. Sillo (1), L. Giordano (1,2), M. M. Garbelotto (3), (1) University of Torino / DISAFA, Grugliasco, ITALY; (2) University of Torino / AGROINNOVA, Grugliasco, ITALY; (3) UC Berkeley, Berkeley, CA, USA

The North American forest pathogen Heterobasidion irregulare was introduced in an area of Italy marginally occupied by the congeneric $H$. annosum. The observed dominance of the invasive species is linked to its higher saprobic and sporulation potentials. H. irregulare $x$ H. annosum hybrids are common in the area of sympatry. Three new studies on interspecific interactions demonstrates: 1-Comingling of the two species on wood results in increased sporulation by H. irregulare; 2-Hybrids carrying the irregulare mitochondrion are more fit than those carrying the annosum mitochondrion; 3Genic introgression between the two species is quantitatively and qualitatively different. Introgression into $\mathrm{H}$. irregulare is massive, but genomic 
analyses shows that introgressed genes are putatively associated with regulatory functions. Conversely, introgression from $H$. irregulare into $H$. annosum is less preponderant, and introgressed genes are putatively involved in various functions associated with saprobic processes and secretory Golgi-related pathways. We predict that should the invasive species spread to areas in Europe where significant populations of $H$. annosum are present, its rate of establishment and local spread may be more relevant than that currently observed in central Italy. Thus, species interactions facilitate the invasion, and hybridization may result in more fit genotypes bearing mitochondria and most of genes of $H$. irregulare, and regulatory genes of $H$. annosum.

Novel source of nematode resistance for Australian sugar industry

S. A. BHUIYAN (1), B. Croft (2), M. Cox (3), P. Jackson (4), (1) Sugar Research Australia Limited, Woodford, AUSTRALIA; (2) SRA, Woodford, AUSTRALIA; (3) SRA, Bundaberg, AUSTRALIA; (4) CSIRO, Brisbane, AUSTRALIA

Root-knot (Meloidogyne javanica) and root-lesion nematodes (Pratylenchus zeae) are the two most important nematode pests of sugarcane in Australia. No nematode resistant varieties are available for the Australian sugar industry. An Australian collaborative introgression program with Chinese institutes has used new sources of wild relatives of sugarcane (Erianthus spp., Saccharum spontaneum and S. robustum), to generate over 100 new hybrid sugarcane families. In 2011, a project was commenced to test the clones from these introgression crosses for nematode resistance; since then approximately 400 clones have been screened. For both nematodes, the basic E. arundinaceus and $S$. spontaneum clones were significantly more resistant than the clones from the core program (Prob $<0.01$ ). In contrast, the basic $S$. robustum clones were significantly more susceptible to root knot nematode than the core clones but significantly more resistant to root-lesion nematode (Prob $<0.01$ and Prob $<0.05$ respectively). With each successive generation of back crossing, using E. arundinaceus and S. spontaneum hybrids, nematode resistance has decreased. However, some individual clones from advanced generations have shown resistance to both nematodes, indicating segregation of the resistance. Individual nematode-resistant clones are being further tested in the glasshouse and field, and may prove to be useful commercial varieties or could be used as parents in the plant breeding program.

Asexual evolution and population sensitivity changes over time in Sclerotinia homoeocarpa in the absence of fungicide pressure G. JUNG, University of Massachusetts, Amherst, MA, USA

Dollar spot, caused by Sclerotinia homoeocarpa ("sterile" multinucleated ascomycete) is the most prevalent and economically important turfgrass disease in North America. Increasing levels of resistance to four single-site mode of action fungicide classes and multi-drug resistance, coupled with environmental and financial constraints, has left fewer options for managing dollar spot. Better understanding of the dynamics and evolution of resistance in S. homoeocarpa populations is urgently needed to improve dollar spot management strategies. In my talk, dynamics of three different field populations with benzimidazole, demethylation inhibitor, and multiple fungicide resistance in the presence and absence of fungicide pressures will be discussed.

Integration of postharvest fungicides and fruit sanitation treatments to optimize decay control and address food safety concerns J. E. ADASKAVEG, Department of Microbiology and Plant Pathology, University of California, Riverside, CA, USA

Aqueous postharvest recycling fungicide-drench applications are one on the most effective strategies for preventing fruit decay at low use rates and fruit residues. Sporulation control, however, is optimized when fungicides are used in fruit coatings that also improve fruit appearance and reduce water loss during storage/marketing. Thus, staged or sequential fungicide applications during postharvest processing-grading of fruit result in the highest level of decay management. With the establishment of the Food Safety Modernization Act (FSMA) in the United States, sanitation of recycling fungicide drenches is mandatory due to the potential contamination with food-borne human pathogens. This has caused the potential elimination of recycling fungicide-drench treatments due to sanitizer incompatibility with fungicides and/or worker safety issues from volatile odors. Thiabendazole, fludioxonil, azoxystrobin, and difenoconazole are compatible with sodium hypochlorite; whereas imazalil, pyrimethanil, and natamycin are not. These fungicides except natamycin are compatible with peroxyacetic acid. Both sanitizers, however, are odoriferous. Acidification ( $\mathrm{pH} 3.5)$ with natural organic acids and food-grade surfactants is compatible with all postharvest fungicides, non-odoriferous, non-phytotoxic, and toxic to human-borne pathogens. Thus, sanitation of recycling fungicide-drenches by acidification meets FSMA requirements and allows highly effective decay control.

Once upon a time: A ten-year history of biocontrol against Fusarium Head Blight

S. SARROCCO (1), A. Zapparata (2), R. Baroncelli (1), G. Vannacci (1), (1) University of Pisa, DISAAA-a, Pisa, ITALY; (2) University of Pisa, Pisa, ITALY

In view of developing novel, eco-friendly and sustainable tools to reduce the incidence of Fusarium Head Blight (FHB) on wheat and prevent mycotoxins from entering the food chain, focus of our research activity has been, for many years, the use of beneficial fungi against Fusarium graminearum, one of the main causal agents of the disease. Trichoderma gamsii T6085 is a well known mycoparasite, can positively control pathogen's growth and mycotoxin (DON) production and reduce FHB incidence in field. T6085 was also used together with a Fusarium oxysporum isolate in competition tests for natural substrates, including cultural debris, where $F$. graminearum growth, trichothecene production and perithecia development were significantly reduced. One of the most interesting and promising aspects of this test was that, when used together, no antagonistic or competitive interactions between the two beneficial fungi were registered, leaving open the possibility of a combined use in a multitrophic approach. A transcriptomic analysis was recently performed to study differentially expressed genes by $T$. gamsii $\mathrm{T} 6085$ and $F$. graminearum during the sensing phase (i.e., before physical contact) of their mycoparasitic interaction. Finally, the CRIPR-Cas9 technique is under investigation in $T$. gamsii T6085 and $F$. graminearum to edit genes putatively involved in plant/pathogen and antagonist/pathogen interaction in order to further investigate aspects connected with the biocontrol of FHB.

Track emerging late blight in the US and South America using a disease alert and surveillance systems and population genomics J. B. RISTAINO (1), S. Restrepo (2), (1) NC State University, Raleigh, NC, USA; (2) Universidad de los Andes, Bogota, COLOMBIA

Phytophthora infestans caused historic disease in Ireland and is a threat to food security globally. We have tracked the recent and historic genotypes of $P$. infestans using multilocus genotyping, next generation sequencing, geospatial analytics and mapping. The same unique multilocus genotype, named FAM-1, caused both US and European historic outbreaks and was found in the oldest samples from Colombia. In 2009, potato and tomato late blight epidemics in the US were the worst in modern history due to widespread inoculum distribution from infected tomato transplants. A surveillance system called USAblight.org was deployed in the US to report disease outbreaks, pathogen genotypes and alerts growers of disease. Today the US-23 lineage is dominant in the US. Most recent lineages of $P$. infestans have emerged out of Mexico, however US-1 and US-23 share allelic diversity with South American (SA) lineages. Only a few clonal lineages of the pathogen have been found on Solanum tuberosum in the Andean countries, however the 
pathogen has been understudied there. The Latinblight network under development in SA will provide a shared database and will enable more studies of gene flow of the pathogen within the Americas and emergence of novel Phytophthora species. This will help clarify the phylogenetic origin of Phytophthora species associated with distinct plants within the Solanaceae family in South America, including the recently described species $P$. betacei. Furthermore it will allow tracking the migration routes and host range of these species.

International Late Blight Networks - a successful collaborative initiative

H. SCHEPERS (1), J. Grønbech Hansen (2), A. Lees (3,4), (1) Wageningen University \& Research, Luttelgeest, NETHERLANDS; (2) Aarhus University, Tjele, DENMARK; (3) James Hutton Institute, Dundee, SCOTLAND; (4) The James Hutton Institute, Dundee, SCOTLAND

EuroBlight, a European network of scientists, advisors and representatives from agrochemical and potato breeding companies meets every $2^{\text {nd }}$ year (www.euroblight.net) to coordinate and discuss late blight research. During the workshops, research results are shared and current challenges and opportunities for late blight control are identified. EuroBlight is organized in working groups that are active in between the workshops. Key activities are: i) monitoring of late blight SSR based genotypes across Europe ii) maintenance and updating of the EuroBlight fungicide table based on coordinated fungicide efficacy trials and iii) development of late blight decision support systems. Data and web tools are managed by a Potato Late Blight Toolbox that controls databases and the generation of interactive map tools and charts available on the EuroBlight web site. The recognised success of EuroBlight has inspired the creation of other international late blight networks, notably USABlight (www.usablight.org), Tizón Latino in Latin America (www.tizonlatino.wordpress.com) and more recently AsiaBlight. Collaboration between these networks through joint research and information sharing makes it possible to improve control of late blight on a global scale. One important aspect for collaboration has been the harmonization of protocols for determining the genotype of Phytophthora infestans isolates and linking this to phenotype, allowing an improved understanding of the pathogen population worldwide. The network of networks can be used to generate the global genetic landscape of $P$. infestans, understand drivers for spread and evolution and to develop DSSs adapted to regional conditions, including production of training and extension material for (small holder) farmers in potato growing regions world-wide.

Potato virus $Y$ evolves to overcome strain-specific resistance in potato: Rapid shift to recombinant virus strains in the U.S. potato A. V. KARASEV, University of Idaho, Moscow, ID, USA

Potato virus $Y$ (PVY) exists as a complex of strains, including multiple recombinants. Recombinant strains have been spreading in the U.S. for the past several years, but the reasons behind this spread were not clear. To document and assess this spread between 2011 and 2017, strain composition of PVY isolates circulating in the Columbia Basin potato production area was determined from hundreds of seed lots of various cultivars. The proportion of nonrecombinant $\mathrm{PVY}^{\mathrm{O}}$ isolates circulating in Columbia Basin potato dropped ten-fold during this period. This decrease in $\mathrm{PVY}^{\mathrm{O}}$ was concomitant with the two-fold rise of the recombinant $\mathrm{PVY} \mathrm{N}^{\mathrm{N}-\mathrm{Wi}}$ strain incidence. Greenhouse and semi-field experiments suggested that hypersensitive resistance (HR) genes present in the most popular potato cultivars may be responsible for the selection against $\mathrm{PVY}^{\mathrm{O}}$ strain and for the concomitant spread of recombinants in potato fields.

An endolysosomal pathway controls cytoplasmic accumulation of helper immune receptors in an NLR network C. DUGGAN (1), C. H. Wu (2), C. Peillex (3), L. Derevnina (2), S. Kamoun (2), T. Bozkurt (1), (1) Imperial College London, London, UNITED KINGDOM; (2) The Sainsbury Laboratory, Norwich, UNITED KINGDOM; (3) ENS Lyon, Lyon, FRANCE

In asterids, a complex signalling network of nucleotide-binding leucine-rich repeat (NLR) proteins mediates resistance to viruses, nematodes, bacteria and oomycetes. In this network, the helper NLR NRC4 pairs with sensor NLRs, such as Rpi-blb2, Mi-1.2 and Rx, to mediate hypersensitive response (HR) and immunity. The molecular mechanisms that regulate NRC4 protein turnover are uncharacterized. We hypothesized that NRC4 protein levels must be tightly regulated to ensure an appropriate level of immunity. To investigate this, we identified NRC4 interactors by mass spectrometry. We found that NRC4 associates with components of vesicle transport regulators and vacuolar sorting adaptors implicated in lysosomal degradation. Knocking-down some of these interactors altered NRC4 protein stability and the HR triggered by an autoactive NRC4 mutant. Overexpression of some of these proteins suppressed NRC4 triggered HR, consistent with their putative roles in promoting NRC4 degradation. Notably, this pathway controlled protein accumulation of other NRC helpers but not NLRs outside the NRC clade. Our results implicate a vacuolar degradation pathway in regulating helper NLR protein accumulation and immunity. This could enable plants to avoid unwarranted immune activation under normal conditions and maintain optimal levels of helper-sensor NLRs in a complex network. Strategic fine-tuning of this pathway could improve broad-spectrum disease resistance to diverse plant pathogens.

Trichothecene diversity and role of plant detoxification enzymes in host resistance

G. ADAM, H. Michlmayr, K. Twaruschek, J. Welsch, M. Siller, G. Wiesenberger, E. Varga, A. Malachova, M. Piatkowska, F. Berthiller, M. Doppler, R. Schuhmacher, University of Natural Resources and Life Sciences, Vienna, Tulln, AUSTRIA

Trichothecenes produced by phytopathogenic fungi are a major agricultural problem. These mycotoxins act as inhibitors of eukaryotic protein synthesis and play a role as fungal virulence factors. The ability of plants to counteract toxicity is difficult to study, since detoxification is typically mediated by members of large gene families. Individual members of the gene family 1 of UDP-glycosyltransferases (UGT, comprising about 160-180 genes in diploid plants) are able to convert deoxynivalenol (DON) and other trichothecenes into the respective glucosides. Transgenic wheat or Brachypodium overexpressing such UGT genes showed increased Fusarium resistance. Recombinant UGTs revealed large differences in affinity for different substrates. Structural changes allowing to (at least partially) escape detoxification seem to be a driving force in the structural diversification of trichothecenes. For instance, loss of TRI13 function causes a switch from nivalenol (NIV) to DON production in F. graminearum. Barley UGT13248 showed lower affinity for DON than NIV indicating this may be advantageous. Yet, the frequently clustered UGTs can evolve new specificity. For instance, while UGT Bradi5g02780 can only detoxify NIV efficiently, the highly similar Bradi5g03300 has higher affinity for DON. Glutathionemediated detoxification is another trichothecene resistance mechanism, recent progress in the study of the large GST family (glutathione-S-transferases) will be presented.

The Flower and Berry Microbiomes of Wild and Cultivated Cranberries in Southeastern Massachusetts G. Ebadzadsahrai (1), A. Harrison (1), M. Mohabbatizadeh (2), S. SOBY (1), (1) Midwestern University, Glendale, AZ, USA; (2) Mason General Hospital, Shelton, WA, USA

Healthy plants host large and diverse populations of bacteria, but much of the attention that has been paid to plant-associated bacteria has centered on pathogenic bacteria, and the soil and rhizosphere microbiome. We examined bacterial populations on cranberry flower and fruit surfaces using culture- 
dependent and culture-independent approaches. Our results show that flowers and fruits harbor bacterial communities with limited diversity, but that the communities on each organ are significantly different. Proteobacteria, Firmicutes, Actinobacteria and Bacterioidetes were the most dominant bacterial phyla on both flowers and berry surfaces. Tenericutes, a phylum that includes the genus Phytoplasma, were observed in appreciable numbers only in flowers. The most represented genera included Pseudomonas, Burkholderia, Acinetobacter, Sphingomonas, Candidatus Phytoplasma, Bacteroides, Salinibacterium, Escherichia, and Bacillus. Culture-dependent methods indicated that there are several notable shifts in population structure associated with maturation of berries on vines over the winter. Among the Gram negative bacteria, only g-proteobacteria (Pseudomonas, Pantoea, Ewingella, Erwinia) and b-proteobacteria (Xylophilus, Delftia) were present. Gram positive bacteria were limited to Firmicutes (Bacillus, Lysinibacillus, Paenibacillus), and Actinobacteria (Curtobacterium, Leifsonia).

Inatreq ${ }^{\mathrm{TM}}$ active: A novel natural product based fungicide for control of major diseases in cereal crops A. LEADER (1), G. M. Kemmitt (2), J. P. Steckler (3), (1) DowDuPont Agriculture Division, Cambridge, UNITED KINGDOM; (2) DowDuPont Agriculture Division, Abingdon, UNITED KINGDOM; (3) DowDuPont Agriculture Division Switzerland SA, CH-8810 Horgen, SWITZERLAND

Inatreq ${ }^{\mathrm{TM}}{ }^{1}$ active (Fenpicoxamid) is the first molecule from a new class of fungicides (picolinamides). Inatreq is derived from the natural product UK-2A which is produced by fermentation of an actinomycete (Streptomyces sp. 517-02) and then undergoes a minor synthetic modification which adds stability and formulation benefits. Inatreq is active against a range of ascomycetes and basidiomycetes and is particularly effective against Zymoseptoria tritici (Septoria tritici blotch), a serious pathogen in temperate wheat growing regions. Inatreq has residual properties and forms a stable deposit on the leaf surface that enables continuous uptake of Inatreq into plant tissue so offering both strong protectant and curative activity. The unique biochemical action of Inatreq involves conversion back to UK-2A in the presence of fungi and/or inside plant tissue. UK-2A inhibits fungal mitochondrial respiration by binding at the Qi ubiquinone reductase site of cytochrome $b c 1$. This represents the first new target site within the cereal fungicide segment for over a decade. In recent years the control of $Z$. tritici in Europe has been challenged following resistance development to some current market solutions. Significantly, Inatreq shows no target site cross-resistance to key cereal fungicide chemistries and is effective on $Z$. tritici resistant isolates. Inatreq will offer cereal growers effective control of cereal diseases with related yield and quality benefits, as well as a new resistance management tool. However, as Inatreq is a single site inhibitor, this new active ingredient will itself require an effective resistance risk management strategy to preserve its long term efficacy. ${ }^{1}$ Trademark of The Dow Chemical Company ("Dow") or an affiliated company of Dow.

Current status of mango malformation disease and its causal agents in Malaysia

H. Rodzali, L. Zakaria, H. Nagao, N. M. I. MOHAMED NOR, Universiti Sains Malaysia, Minden, MALAYSIA

Mango malformation disease (MMD) was first reported in India in 1891 and has progressively spread to other mango growing countries across different continents. MMD is an economically important disease to the mango industries as it infects inflorescences and vegetative parts of mango tree. The interruption on the plant canopy and floral developments reduces the number of fruit production. More than one species of Fusarium was reported to be the causal agent of MMD from different regions. In 2013, Fusarium proliferatum was reported to be associated with MMD in Malaysia. However, pathogenicity was not performed, thus the causal agent of mango malformation disease in Malaysia remains unclear. The objectives of this study are to conduct a field survey on the occurrence of MMD, and to determine the causal agent of MMD in Malaysia. A survey on the occurrence of MMD in peninsular Malaysia was conducted focusing on mango orchards and nurseries. Throughout the peninsular Malaysia, only two mango orchards were found to be infected with this disease at inflorescence stage. A total of 83 isolates of Fusarium spp. were collected from the malformed tissue. The identification of Fusarium was based on the morphology and molecular methods. There were three dominant species isolated from the malformed inflorescences which were $F$. mangiferae, $F$. concentricum, and $F$. proliferatum. The pathogenicity of these isolates was being conducted on the inflorescence tissue and 6 month old mango seedlings of the Chokkanan variety. Our preliminary results showed that $F$. proliferatum causes the mango flowers becoming necrotic and fall off. The malformation symptoms have not yet been observed from the pathogenicity test. This study will resolve the problem on determining the causal agent of MMD in Malaysia.

Detection of threatening emerging pathogens in maize and wheat seed: Phytosanitary challenges, regulations and solutions M. MEZZALAMA (1), P. L. Kumar (2,3), (1) CIMMYT, Texcoco, MEXICO; (2) International Institute of Tropical Agriculture, Ibadan, NIGERIA; (3) International Institute of Tropical Agriculture (IITA), Ibadan, NIGERIA

The Maize chlorotic mottle virus (MCMV) responsible for maize lethal necrosis in East Africa since 2011 and Magnaporthe oryzae Triticum (MoT), responsible for wheat blast in the tropical parts of South America since 1985 and in Bangladesh since 2016, have emerged as serious threat to global maize and wheat production, respectively. Seed transmission was suspected to be the mechanism of intercontinental spread of both these pathogens. Reliable testing procedures for pathogen detection in seed lots have become a high priority for enforcing effective phytosanitary controls during seed exchanges. In this study we present development of seed testing methods and regulatory procedures for declaring seed lots and germplasm meant for international exchange free from MCMV and MoT for commercial and research purposes. Enzyme-linked immunosorbent assay (ELISA) for MCMV in maize and a polymerase chain reaction (PCR) with MoT specific primers in wheat were found to be the most reliable and cost-effective methods for pathogen detection in seed lots. Challenges to diagnostic test development due limited knowledge on rate and mode of seed transmission of MCMV, and occurrence of different MoT pathotypes will be presented.

Shifting populations of blackleg causing organisms: Significance and possible control strategies J. VAN DER WOLF, Wageningen University and Research, 6700 AA Wageningen, NETHERLANDS

An increasing number of (sub)species within the group of soft rot Pectobacteriaceae (SRP) are found to cause potato blackleg. Consecutively Pectobacterium atrosepticum, Dickeya dianthicola, P. brasiliense, D. solani and P. parmentieri were identified as causative agents. The prevalence of the SRP-variants varies in time and place. Recently, in North-America and Australia, severe blackleg outbreaks with aggressive strains of $D$. dianthicola were found. In Australia, the pathogen likely originated from infected Dahlia's. In Europe, initial infections with $D$. solani were probably also derived from infected ornamentals, indicating the risks for the introduction of new SRP variants in potato from other hosts. The population structure of blackleg causing organisms can change rapidly as was experienced in the Netherlands. In 2000, D. solani was found for the first time in potato and from 2006 till 2012 it was the major causative agent of blackleg. Since 2012, P. brasiliense became the dominant blackleg causing pathogen. The introduction of a new SRP variant may initially result in an increasing blackleg prevalence and damage, but there are indication that in time, the new variants become less aggressive. Control of blackleg is still based on the use of pathogen-free seed, less susceptible varieties, on hygiene and proper cultivation practices that include avoiding plant damage, oxygen depletion in soil, and cross-contaminations. The use of biocontrol agents for field applications is still in an explorative phase. It was found that seed lots can be highly suppressive against blackleg pathogens. There is a search ongoing for treatments that can enhance suppressiveness. 
Changes in soil microbial communities associated with Armillaria root disease of western white pine (Pinus monticola)

B. M. LALANDE (1), N. B. Klopfenstein (2), M. S. Kim (3), Z. Abdo (1), J. Stewart (4), (1) Colorado State University, Fort Collins, CO, USA; (2) Rocky Mountain Research Station, USDA Forest Service, Moscow, ID, USA; (3) Dept. of Forestry, Environment and Systems, Kookmin University, Seoul, SOUTH KOREA; (4) Department of Bioagricultural Sciences and Pest Management, Colorado State University, Fort Collins, CO, USA

In forests, soil interactions among Armillaria species, microbial communities, and roots may influence tree growth and survival. Two fungal species, $A$. solidipes (highly virulent) and A. altimontana (less virulent), frequently co-occur in forests of inland northwestern USA. Understanding microbial communities associated with each Armillaria species may provide novel insights for managing Armillaria root disease. This study aims to identify soil microbes and their community structure around A. solidipes, A. altimontana, and western white pine (Pinus monticola). Results of a field study revealed significant changes in microbial communities associated with two different Armillaria spp. Diverse and beneficial bacterial communities were highly related to the presence of $A$. altimontana, whereas trees infected with $A$. solidipes had more diverse soil fungal communities. In a greenhouse experiment, 74 western white pine seedlings were grown in five treatments: inoculated with either $A$. solidipes or $A$. altimontana, single inoculations with a delayed alternate species inoculation, simultaneous dual inoculations, and control. DNA sequences of ITS-2 and 16S rDNA were used for soil metagenomic analyses. Seedling diameter at root collar and height were recorded. Results from this study will help develop novel management approaches for suppressing Armillaria root disease by favoring beneficial microbial communities with potential biocontrol activity against Armillaria pathogens.

Wheat stripe rust: Are recent pandemic races associated with the sexual cycle of the pathogen?

M. S. Hovmøller (1), J. RODRIGUEZ ALGABA (2), T. Thach (1), S. Ali (3), A. F. Justesen (4), (1) Aarhus University, Slagelse, DENMARK; (2) Aarhus University, 4200, DENMARK; (3) University of Agriculture, Peshawar, Peshawar, PAKISTAN; (4) Danish Inst of Agricultural Sciences, Slagelse, DENMARK

A changing climate and increasing travel and trade may be important drivers for inter-continental spread of yellow (stripe) rust to new areas and spread of new races to previously resistant crop varieties. The latter was the case when the NW European Puccinia striiformis population in 2011 was replaced by new races of non-European origin. The 'Warrior' race was present at many locations in the first year of detection, whereas the 'Kranich' race was predominant only in few regions. Presence of several multi-locus genotypes of both races was consistent with a hypothesis of aerial spread of spores from genetically diverse source populations. Both races produced more telia on the wheat host than previously detected races in Europe, and teliospores were able to germinate and produce basidiospores infecting the alternate host, Berberis vulgaris. Sexual reproduction was completed under experimental conditions by recovery of progeny isolates from wheat inoculated by aeciospores from $B$. vulgaris. Based on informative microsatellite markers and reference isolates from six continents, it was concluded that the 'Warrior' and 'Kranich' races originated from the centre of diversity of the yellow rust fungus in the near-Himalayan region. The study illustrated the potential role of sexual reproduction of wheat rust fungi, serving as a reservoir for new races replacing distant clonal populations, and causing disease epidemics at continental scales on previously resistant crop varieties.

Transcriptome and genome analyses of the biocontrol fungus Clonostachys rosea highlights toxin tolerance as a key biocontrol trait K. Nygren (1), M. Dubey (1), A. Zapparata (2), M. Iqbal (1), G. Tzelepis (3), M. Brandström Durling (1), D. F. Jensen (1), M. KARLSSON (1), (1) Dept. Forest Mycology and Plant Pathology, Swedish University of Agricultural Sciences, Uppsala, SWEDEN; (2) University of Pisa, Pisa, ITALY; (3) Dept. Plant Biology, Swedish University of Agricultural Sciences, Uppsala, SWEDEN

The biological control fungus Clonostachys rosea is able to detoxify the antifungal Fusarium mycotoxin zearalenone, and is tolerant towards a wide range of microbial secondary metabolites. We hypothesize that the mycoparasitic lifestyle of $C$. rosea have resulted in evolution of tolerance mechanisms against toxic secondary metabolites produced by the fungal prey, $C$. rosea itself and the surrounding soil microbiota. A transcriptomic study of $C$. rosea interacting with the fungal plant pathogens $F$. graminearum and Botrytis cinerea showed that $61 \%$ of all induced genes were predicted to encode ATP-binding cassette (ABC) and major facilitator superfamily (MFS) membrane transporters, while $12 \%$ were predicted to encode proteins involved in biosynthesis of secondary metabolites. Detailed evolutionary analyses showed that a majority of the membrane transporter genes belonged to families evolving under selection for increased paralog numbers, with predicted functions in multidrug resistance. Deletion of the MFS transporter gene $m f s 464$ resulted in mutants with increased $(P=0.001)$ growth inhibitory activity against $F$. graminearum, providing evidence for a function in interspecific fungal interactions. Deletion of the $\mathrm{ABC}$ transporter genes $a b c G 5$ and $a b c G 29$ resulted in mutants that failed $(P=0.001)$ to protect barley seedlings against fusarium foot rot disease in growth chamber tests. In summary, our data suggest that membrane transporters play an important role in the biology of $C$. rosea, by providing tolerance towards secondary metabolites produced by the fungal prey or $C$. rosea itself.

Aflatoxin producers in Mozambique include a distinct $\mathrm{S}$ morphology taxon with high capacity to produce aflatoxins in Maize and Groundnut L. R. L. ARONE (1), J. Augusto (2), R. Bandyopadhyay (3), P. J. Cotty (4), (1) University of Arizona, Tucson, AZ, USA; (2) Intl Inst of Tropical Agriculture, Nampula, MOZAMBIQUE; (3) International Institute of Tropical Agriculture, Ibadan, NIGERIA; (4) USDA-ARS, University of Arizona, Tucson, AZ, USA

Aflatoxins are potent mycotoxins produced by several Aspergillus species. In Mozambique, aflatoxins prevent groundnuts from entering export markets and cause high rates of liver cancer. High frequencies of Aspergillus with S morphology were discovered near Nampula, where human exposure to aflatoxins has been historically severe. To determine taxonomic affiliations of the Nampula fungi, phylogenetic analyses were applied to sequences from genome regions harboring genes for either the aflatoxin transcription factor (aflR, 1.8 kb), nitrate reductase (niaD, $2.2 \mathrm{~kb}$ ), an MFS-transporter (aflT, 0.9 $\mathrm{kb})$ or a cytochrome $\mathrm{P} 450$ monooxygenase $(\operatorname{cyp} A, 3.1 \mathrm{~kb})$. S morphology isolates $(\mathrm{n}=448)$ were resolved into seven distinct taxa, with the majority $(72 \%)$ clustering into a distinct and novel taxon sister to the previously described unnamed taxon $\mathrm{S}_{\mathrm{BG}}$. Another distinct taxon, sister to the lethal aflatoxicosis fungi from Kenya, contained $20 \%$ of isolates while $8 \%$ were identified as $A$. minisclerotigenes. Although all S morphology fungi produced high aflatoxin concentrations, the newly discovered and most common taxon produced the highest aflatoxin concentrations (up to $62.5 \mathrm{mg} / \mathrm{kg}$ in maize and up to $17.6 \mathrm{mg} / \mathrm{kg}$ in groundnut); A. minisclerotigenes produced the lowest concentrations (up to $1.3 \mathrm{mg} / \mathrm{kg}$ in maize). Optimal selection of atoxigenic biocontrol agents should take into consideration effectiveness against the new taxa which are potent causal agents of aflatoxin contamination.

Zymoseptoria tritici histone modifications distinguish core and accessory chromosomes and play an important role in genome stability M. MOELLER (1,2), K. Schotanus (3), J. L. Soyer (2,4), M. Freitag (5), E. H. Stukenbrock (1,2), (1) Christian-Albrechts University of Kiel, Environmental Genomics, Kiel, GERMANY; (2) Max Planck Institute for Evolutionary Biology, Plön, GERMANY; (3) Duke University Medical Center, Department of Molecular Genetics and Microbiology, Durham, NC, USA; (4) UMR BIOGER, INRA, AgroParisTech, Université Paris-Saclay, Thiverval Grignon, FRANCE; (5) Oregon State University, Department of Biochemistry and Biophysics, Corvallis, OR, USA 
Zymoseptoria tritici is a fungal pathogen of wheat. The genome of the reference isolate comprises 21 chromosomes of which eight are accessory. These chromosomes are not key determinants for virulence, as in other fungal pathogens, however, they are partly conserved among distinct Zymoseptoria species. Accessory chromosomes are highly instable during meiosis and mitosis, transcriptionally repressed and show enrichment of repetitive elements and heterochromatic histone marks. To elucidate if heterochromatic histone modifications have an impact on chromosome stability, we created deletion mutants of the methyltransferases responsible for $\mathrm{H} 3 \mathrm{~K} 27 \mathrm{me} 3$ and $\mathrm{H} 3 \mathrm{~K} 9 \mathrm{me} 3$. H3K27me3 is specifically associated with accessory chromosomes and subtelomeric regions in Z. tritici, while H3K9me3 is associated with repetitive elements. We combined experimental evolution, genetic and highresolution microscopic analyses to follow the impact of these deletions on chromosome and genome stability. We used ChIP-seq, genome sequencing and RNA-seq to compare changes in chromatin and genome structure and differences in gene expression between mutant and wild type strains. The loss of H3K9me3 results in dramatic chromatin reorganization, transposable element activation, genome rearrangements, formation of "neochromosomes" and increased accessory chromosome instability. Loss of $\mathrm{H} 3 \mathrm{~K} 27 \mathrm{me} 3$, however, has little effect on chromatin organization and transposon control, but increases stability of the accessory chromosomes. We conclude that $\mathrm{H} 3 \mathrm{~K} 9 \mathrm{me} 3$ strongly impacts chromatin and genome organization and that H3K27me 3 has an important role in stability of accessory chromosomes. Epigenetic regulation is an important driver of genome evolution in this fungus and can contribute to rapid adaptation.

Resistance mechanisms of SDHI fungicides in Sclerotinia homoeocarpa field isolates

G. JUNG (1), J. T. Popko (1), H. Sang (2), J. Lee (3), (1) University of Massachusetts, Amherst, MA, USA; (2) Michigan State University, East Lansing, MI, USA; (3) UMass Amherst, Amherst, MA, USA

Dollar spot, caused by S. homoeocarpa, is the most economically important disease on turfgrass. Succinate dehydrogenase inhibitor (SDHI) fungicides have been widely applied to control dollar spot. Updated resistance mechanisms in golf course isolates from Japan and Rhode Island are presented. The Japanese isolates harbor a H-to-Y mutation at position 267 or silent mutation at 181 in the SdhB gene and a G-to-R mutation at 150 in the SdhC gene. The Rhode Island isolates harbor a mutation at position G91R or G159W in the SdhC gene. The SdhB H267Y mutation was functionally validated and exhibited high resistance to boscalid, moderate resistance to penthiopyrad and fluxapyroxad, weak resistance to isofetamid, and a hypersensitive response to fluopyram. The SdhC G91R mutation was functionally validated and exhibited high resistance to boscalid, fluxapyroxad and penthiopyrad, moderate resistance to isofetamid, and weak resistance to fluopyram. In addition, a single Japanese isolate with the SdhC G150R mutation had a similar response to the SdhC G91R isolate. In contrast, the Rhode Island isolate with both G159W and silent mutations had a similar SDHI resistance profile to the $\mathrm{H} 267 \mathrm{Y}$ isolate group. A silent mutation was also found at position $181 \mathrm{in} \mathrm{SdhB}$ and no other mutations that cause an amino acid substitution were detected in the SdhB, SdhC and SdhD subunits. Updated results indicate that the SDHI cross- or negative-resistance profile is dependent on the target gene mutation (i.e. among subunits and mutation position within subunit) and considerable variability exists.

Integration of light and jasmonate perception in the control of growth and defense

C. BALLARE, IFEVA, University of Buenos Aires-CONICET, Buenos Aires, ARGENTINA

Light signals, perceived through informational photoreceptors, are used by plants to detect and respond to the proximity of competitors. Low red:farratios (R:FR) in the canopy light reduce the proportion of phytochrome B (phyB) molecules that are in the active form, and promote the synthesis of growth-related hormones, including auxin and gibberellins, which in turn stimulate shoot elongation and other shade-avoidance responses. At the same time, recent research demonstrates that phyB is an important modulator of the two principal hormonal pathways that regulate plant immunity against herbivores and pathogens, i.e. the jasmonic-acid (JA) and the salicylic-acid pathways (SA). Low R:FR ratios down-regulate JA- and SA-induced defense responses. This down-regulation is thought to help the plant to efficiently redirect resources from defense to rapid growth under conditions of intense competition. In this presentation, I will discuss recent advances in the understanding of the mechanisms that link phyB with JA signaling, and explore the consequences for direct and indirect defenses. Unveiling the molecular links between photoreceptors and the regulators of plant immunity is important to generate a mechanistic framework to understand how plants deal with resource allocation trade-offs under natural conditions. This mechanistic understanding can be very useful to guide breeding programs aimed at increasing plant resistance to pests and pathogens in cultivated species.

Wheat Blast: Unveiling epidemiological aspects

M. FERNANDES (1), K. B. Mills (2), P. A. Paul (3), L. V. Madden (3), (1) Embrapa Wheat, Passo Fundo, BRAZIL; (2) Ohio State University, Wooster, OH, USA; (3) The Ohio State University, Wooster, OH, USA

Wheat blast is an emerging disease in South America and Asia caused by the Triticum pathotype of Magnaporthe oryzae (MoT). Wheat blast is a serious disease capable of causing severe losses, especially during warm and humid weather conditions. Here, we report a weather based model to predict wheat blast outbreaks. Specifically, uncommonly humid and warm weather during a 60-day period preceding wheat heading is considered key drivers of inoculum build up and airborne spores from regional inoculum sources in the surroundings. A day favoring infection was conditioned to rules relating temperature and relative humidity. Successful daily infection was conditioned to the inoculum size and density of spores in the air. The risk model has been successfully applied to Brazil and Bangladesh conditions. Although MoT has not yet been reported in North America, the closely related M. oryzae Lolium pathotype (MoL) is commonly found in wheat growing regions and could pose a threat under certain environmental conditions. Multifactorial experiments were conducted under controlled conditions to evaluate the effects of different combinations of temperature, relative humidity duration, inoculum concentration, and growth stage. Results showed that the odds of a spike becoming infected was significantly $(\mathrm{P}<0.05)$ greater when inoculated at early heading than at full head emergence, anthesis, or early grain fill. However, once infected, the rate of blast severity increase per unit increased in high RH duration was significantly greater $(\mathrm{P}<0.05)$ when infections occurred at Feekes 10.5.1 (anthesis) or 10.5.4 than between Feekes 10.1 and 10.5 . The rate of increase in severity per hour increase in $100 \% \mathrm{RH}$ was significantly greater at 25 and $30 \mathrm{C}$ than at $20 \mathrm{C}$. These results are invaluable for ongoing efforts to assess the risk posted by MoL to US wheat.

Searching for the mechanism that mediates the mefenoxam-acquired resistance phenomenon in Phytophthora infestans and how it is regulated J. GONZALEZ TOBON (1), R. Childers (2), M. Regnier (1), A. Rodriguez (1), W. E. Fry (3), S. Restrepo (1), G. Danies (1), (1) Universidad de los Andes, Bogota, COLOMBIA; (2) Harvard University, Cambridge, MA, USA; (3) Cornell University, Ithaca, NY, USA

Phytophthora infestans, the causal agent of late blight disease of potatoes and tomatoes, is mainly controlled by the use of fungicides. Populations of $P$. infestans that are resistant to commonly used fungicides have already been reported. Aside from natural resistance, several studies have shown that isolates of $P$. infestans that are originally sensitive to the fungicide mefenoxam are able to acquire resistance to this fungicide when exposed to sub-lethal concentrations of it. This phenomenon termed 'mefenoxam-acquired resistance' has been observed in different species of the Phytophthora genus and seems to be unique to mefenoxam. The first objective of this study was to elucidate the molecular mechanism mediating this acquisition of resistance. Given that mefenoxam seems to interfere with the pathogen's RNA polymerase I, both in silico and experimental analyses were carried out to evaluate if its activity might be affected by the phenomenon. Furthermore, genes that are differentially expressed between originally sensitive isolates and those 
same isolates after acquiring resistance were identified and further analyzed. The second objective was to investigate if there is a regulatory epigenetic process, such as small non-coding RNAs or chromatin remodeling, mediating this mechanism. This study will narrow-down all possible hypotheses and generate new questions that might contribute to the comprehension of epigenetic and molecular processes of this pathogen.

Catch my drift? Inoculum detection as a decision aid for agricultural systems

L. D. THIESSEN, North Carolina State University, Raleigh, NC, USA

Regional spread of diseases has been traditionally monitored by and communicated between growers, and continues to be monitored by extension specialists, crop consultants, and through formal, nationwide scouting programs. As technology continues to improve and become accessible, so too does our ability to sample for and detect airborne pathogens. Airborne inoculum sampling is now being commercially used to initiate and time fungicide applications in grape powdery mildew management in the Western United States, and similar methods have been developed for diseases in other crops. In addition to using inoculum detection for fungicide applications, inoculum sampling methods can be used to develop more accurate epidemiological models, to rapidly sample for pesticide-resistant pathogen populations, or assess mycotoxin production potential of airborne inoculum. These tools are shifting crop management to targeted approaches that improve disease management and reduce costs to producers.

Central role of dsRNA in the elicitation of antiviral defenses in plants

M. HEINLEIN, Institut de Biologie Moléculaire des Plantes du CNRS (IBMP-CNRS), Strasbourg, FRANCE

Plant viruses rely on macromolecular assembly and transport pathways for replication and for targeting the plasmodesmata (PD) for cell-to-cell movement. Our team investigates these processes in the context of plant defence responses and is interested to identify mechanisms leading to disease. We showed that mutants affected in the pathogen-recognition receptor (PRR) co-receptor kinase BAK1/SERK3 exhibit increased susceptibility to different RNA viruses, thus indicating a role of pattern-triggered immunity (PTI) in antiviral defense. Interestingly, Arabidopsis plants responded to crude extracts from virus-infected plants, but not to purified virions, showing that the molecules that elicit the antiviral PTI response are produced by the infected cell. Infection by RNA viruses is generally associated with the occurrence of double-stranded (ds)RNAs. Accordingly, we found that PTI is elicited by dsRNA. The application of dsRNAs and the synthetic dsRNA analog poly(I:C) to Arabidopsis plants induces typical PTI responses, including the activation of MPK6 and MPK3, ethylene synthesis and defense gene expression. Moreover, poly(I:C) treatment induces seedling growth inhibition, a phenotype associated with the activation of plant immunity. Consistent with the hypothesis that dsRNAs are perceived as viral pathogen-associated molecular patterns (PAMPs) in plants, dsRNA treatment can protect plants against virus infection. The PTI responses were not impaired in plants lacking dicer-like (DCL) proteins indicating that DCLs do not serve as the dsRNA receptors leading to PTI. The dsRNA receptors involved in the triggering of PTI as well as the downstream mechanisms that restrict virus infection may be identified by future studies.

\section{Metagenomic analysis of the aerial mycobiome of rice paddies}

S. FRANCO ORTEGA (1), I. Ferrocino (2), S. Silvestri (3), I. Adams (4), D. Spadaro (5), N. Boonham (4), M. L. Gullino (1), (1) Agroinnova University of Torino, Grugliasco, Torino, ITALY; (2) Department of Agricultural, Forest and Food Science, University of Turin, Grugliasco, ITALY; (3) Ente Nazionale Risi, ENR, Milano, ITALY; (4) Fera Science Ltd, York, UNITED KINGDOM; (5) DISAFA and AGROINNOVA, University of Torino, Torino, ITALY

Airborne microbiome represent a new fascinating topic that has been understudied in comparison with the microbiota present in other environments. Only a small fraction of the organisms in the atmosphere can be studied using traditional culture-based methods. In this study, the aerial composition of fungi in a rice paddy has been examined along the crop production cycle (from June to September) using a targeted DNA-based method (qPCR) to target Magnaporthe oryzae and Cochliobolus miyabeanus and by high throughput sequencing (HTS) targeting the ITS region. Alpha diversity analysis at the beginning of the trial (June) showed a higher level of complexity than at the end of the trial as well as a higher number of operational taxonomic units (OTUs) that decreased over time. The main taxa identified by HTS where the relative abundance drove the cluster separation as a function of the time and temperature were Alternaria, Magnaporthe, Cochliobolus, Aspergillus, Penicillium and Myrothecium. In parallel, oligotyping analysis on the main OTUs was performed to obtain a sub-OTU identification. The results revealed the presence of several characteristic oligotypes associated with meteorological conditions. In addition, a selective pressure in function of temperature on the air mycobiota composition was clearly evidenced. Indeed, changes in temperature level can drastically affect the mycobiota community structure, with a possible impact on the disease development in rice.

Spatial and compositional diversity in the microbiota of harvested fruits: What can it tell us about biological control of postharvest diseases M. WISNIEWSKI (1), S. Droby (2), J. Norelli (3), S. Freilich (4), (1) Appalachian Fruit Research Station, USDA-ARS, Kearneysville, WV, USA; (2) Agricultural Research Organization, The Volcani Center, Rishon Lezion, ISRAEL; (3) USDA-ARS, Kearneysville, WV, USA; (4) ARO, Rishon LeZion, ISRAEL

Accumulating evidence indicates that the composition of the microbiota inhabiting an organism (both endo- and epiphytically) can have a profound effect on host physiology and defense responses. The role of the microbiota in plant health and physiology, however, is poorly understand, and few studies have focused on temperate fruit trees. A global effort is underway to characterize the endophytic and epiphytic microbiome of apple fruit with the goal of developing a microbial consortium for the management of a wide range of postharvest diseases, and potentially physiological disorders. Spatial studies of the microbiota of apple peel, calyx-end, stem-end, and wounds tissues have revealed distinct compositional differences, as well as differences between organic and conventional management systems. Other studies have indicated a distinct genotype effect on defining the endophytic microbiota in apple shoots. These data suggest that apple pedigree influences the composition of the endophytic microbiota and that the host and microbiome have coevolved to some extent, as suggested in the holobiont concept. The effect of postharvest processes, such as waxing and storage, on the apple fruit microbiome will be presented, as well as a global analysis of the apple fruit microbiome. The implications of greater knowledge of the apple microbiome on disease and cultural management strategies, cultivar breeding, and abiotic stress resistance, will be discussed.

Ecology and evolution of oomycete communities in response to soybean seed treatments Z. NOEL, H. Sang, M. Chilvers, Michigan State University, East Lansing, MI, USA

Soil conservation efforts combined with earlier planting dates has led to increased crop residue and cooler soil at planting. This exposes seeds and developing seedlings to adverse conditions for extended periods of time. To gain a better understanding of soybean seed treatments as oomycete community filters, culture based techniques, evolutionary analysis and genetics are utilized. A high-throughput assay was developed and applied to evaluate the fungicide sensitivity of 81 oomycete species across ten clades to ethaboxam and mefenoxam. Species within three separate Pythium clades all had reduced sensitivity to ethaboxam, suggesting that reduced sensitivity to ethaboxam is inherent and possibly related phylogenetically. Therefore, 
the evolutionary relationship of ethaboxam sensitivity was investigated. Bayesian phylogenetics and ancestral sequence reconstruction of $P y t h i u m ~ \beta-$ tubulin gene trees indicated that species with reduced sensitivity to ethaboxam followed a convergent evolutionary pattern, and had evolved three separate times under two transversion mutations. Heterologous expression of Pythium $\beta$-tubulin in a yeast model system is being used to determine the molecular mechanism of ethaboxam resistance. However, the ecological dynamics of seed treatments on oomycete communities remain difficult to explain. Based on preliminary investigations on isolation and amplicon sequencing data from roots and rhizosphere soils, filtering based on seed treatment chemistry may be subtle and location specific. Currently, other ecological dynamics in combination with seed treatments are being investigated with amplicon sequencing. This information will enhance management of seedling diseases caused by oomycetes in the Midwest.

Development and evaluation of nano-materials for management of copper-tolerant Xanthomonas perforans causing bacterial spot of tomato M. L. PARET (1), A. L. Strayer (2), Y. Y. Liao (2), S. Wright (1), M. Young (3), I. Ocsoy (4), D. Averett (5), G. E. Vallad (6), S. Santra (7), W. Tan (4), J. B. Jones (8), S. M. Olson (9), J. H. Freeman (1), (1) North Florida Research and Education Center, University of Florida, Quincy, FL, USA; (2) University of Florida, Department of Plant Pathology, Gainesville, FL, USA; (3) University of Central Florida, Orlando, FL, USA; (4) University of Florida, Department of Chemistry, Gainesville, FL, USA; (5) EcoActive Surfaces Inc., Pompano Beach, FL, USA; (6) Gulf Coast Research and Education Center, University of Florida, Wimauma, FL, USA; (7) NanoScience Technology Center, Department of Chemistry, University of Central Florida, Orlando, FL, USA; (8) Department of Plant Pathology, University of Florida, Gainesville, FL, USA; (9) Univ of Florida NFREC, Quincy, FL, USA

Bacterial spot of tomato is caused by four Xanthomonas sp. (euvesicatoria, gardneri, perforans and vesicatoria) and is one of the topmost bacterial disease of relevance to tomatoes in the U.S. and worldwide. Currently all strains in Florida are X. perforans and copper-tolerant, leaving few effective compounds for disease management. Thus, finding alternatives to copper bactericides is critical for the tomato industry in Florida. Studies were undertaken from 2010-2017 to develop and evaluate the antibacterial activity of two photocatalytic (light-activated) nanomaterials (titanium dioxide doped with zinc $-\mathrm{TiO}_{2} / \mathrm{Zn}$ or silver - $\mathrm{TiO}_{2} / \mathrm{Ag}$ ), a silver-dsDNA-graphene oxide composite, and three copper composites (core-shell copper - CS-Cu, multi-valent copper - MV-Cu, and fixed quaternary ammonium copper - $\mathrm{FQ}-\mathrm{Cu}$ ) as potential alternatives to copper bactericides. All the tested nanomaterials demonstrated high antibacterial properties against copper-tolerant $X$. perforans strains. These nano-materials were also shown to significantly reduce bacterial spot disease severity in greenhouse and/or field studies compared to non-treated controls and activity was similar or better than coppermancozeb, the grower standard. Nanomaterial composition and properties play important roles in plant safety as well as performance in greenhouse and field conditions. However, successful commercialization of nanomaterial containing bactericides would require clearance of potential regulatory issues.

Atmospheric dispersal of plant pathogens over multiple spatial and temporal scales

D. E. AYLOR (1), F. J. Ferrandino (2), (1) The Connecticut Agricultural Experiment Station, New Haven, CT, USA; (2) Connecticut Agric Exp Station, New Haven, CT, USA

Knowing how far and how fast disease will spread in a region allows growers and land managers to plan. We present a biophysical model for diseases spread by airborne spores that predicts their distance and rate of spread. The model's principal components are distance between fields, meteorological conditions at various scales, dynamics of pathogen buildup, and development of the host. Our model spans four length scales: 1) plant canopy height, 2) field size, 3) regional scale, and 4) continental scale, and four time scales: a) rate of epidemic development, b) infectious and latent period of the pathogen, c) pathogen survival time, and d) the return period of conditions conducive for transport and infection. All eight scales together determine the time for new infections to appear in distant fields, which allows risk to be evaluated for patches of hosts distributed widely across a landscape. At field and farm scales, dispersal is a more-or-less continuous process; while at regional and continental scales dispersal is discontinuous, needing to span the distances between patches of host. This fundamental difference is one reason that wave front speeds are apparently faster on long rather than on short distance scales and it allows for the existence of critical separation distances that can stifle disease spread. The model will be illustrated using apple scab, potato late blight, tobacco blue mold, and stem rust of wheat as examples.

\section{Dynamics of microbial communities associated with broccoli residue and chitin amendments on suppression of Verticillium wilt in three soil types}

K. D. PURI (1), D. P. G. Short (1), P. Inderbitzin (2), D. O. Chellemi (3), K. V. Subbarao (4), (1) University of California, Davis, Salinas, CA, USA; (2) University of California at Davis, Department of Plant Pathology, Davis, CA, USA; (3) Agricultural Solutions, Fernandina Beach, FL, USA; (4) University of California at Davis, c/o U.S. Agricultural Research Station, Salinas, CA, USA

The potential for soil amendments to stimulate and promote disease suppressive microbiomes antagonistic to Verticillium spp. were evaluated in three soil types. Broccoli residue $(10 \% \mathrm{w} / \mathrm{w})$, chitin-based crab meal $(0.2 \% \mathrm{w} / \mathrm{w})$, and a combination treatment were applied to three soils with distinct land use histories: intense agriculture, transition from pasture to agriculture, and native, undisturbed soil. In greenhouse experiments, prokaryote, fungal and oomycete communities; disease severity (DS) on Solanum melongena (eggplant); microsclerotia (MS) density in soil; chitinase activity; and soil physicochemical properties were monitored and analyzed over time. Preliminary prokaryote community analyses indicated that broccoli and crab meal amended soil enriched known fungal antagonists such as Sporocytophaga, Streptomyces, Pseudomonas, Bacillus, and Nannocystis. The same treatments enhanced Bacteroidetes in agricultural and non-agricultural soil but decreased them in transition soil. The DS and MS density significantly decreased over time relative to the unamended control. A detailed analysis of temporal microbial dynamics, and correlations between disease suppressiveness, enzyme activity, MS counts, and soil properties are ongoing. The results indicate that broccoli alone or in combination with crab meal reduces Verticillium wilt severity and enhances suppression-associated microbes, and thus may represent a cultural approach to wilt management in the absence of soil fumigants.

\section{Overview of barriers and successes to transfer of agricultural technology} A. C. FAYAD, Virginia Tech, Blacksburg, VA, USA

Access to information and to agricultural technology remains a major limitation for growers and smallholder farmers to achieve food security, especially in the developing world. The Integrated Pest Management Innovation Lab (IPM IL), a USAID funded program managed by Virginia Tech, improves the livelihoods of smallholder farmers by increasing crop production, nutrition, health, income, and food security. The program develops and disseminates IPM packages for cereal, fruit, legume, and high value vegetable crops. Based on two decades of experience in the developing world, the program has been progressively successful in identifying and overcoming the challenges to technology transfer by collaborating with local scientists, extension services, NGOs, value chain projects, and other organizations, using all available paths to reach farmers in a given country. Farmers' access to agricultural technologies, such as biopesticides, remains a major constraint to the widespread adoption of IPM. In Nepal, the IPM IL collaborated with Agrovets to promote knowledge about the use and benefits of botanicals and biopesticides and access to agricultural technologies, including IPM components. The IPM IL also trained private entrepreneurs on production and quality control of biopesticides to encourage the production of these 
products. This strategy resulted in development of a market for IPM products, privatization of IPM technologies, and a sustainable technology transfer in Nepal.

Detection of multiple oomycetes in metagenomic data by Using E-probe Detection of Nucleic Analysis (EDNA) M. F. PROANO, A. Espindola, C. D. Garzon PhD, Oklahoma State University, Stillwater, OK, USA

Species identification of plant pathogenic oomycetes based on morphology is challenging. Hence, time demanding isolation and ITS sequencing for molecular identification are routinely conducted for diagnostic purposes. Metagenomic diagnostic methods allow identification of multiple pathogens from a single sample without isolation of pure cultures or specific gene-targeted sequencing. E-probe Diagnostic Nucleic acid Analysis (EDNA), a novel method that couples next-generation sequencing and bioinformatics, was used to detect a variety of plant pathogenic oomycetes in metagenomic data. Short pathogen-associated sequences (E-probes), with a range of 60-120 nucleotides, were designed and tested on metadata containing the genomes of a known host and pathogenic oomycetes. E-probes were designed for each pathogen by comparing its genome to the genome of the closest relative available and identifying unique species-specific sequences. To avoid false positives, E-probes with similarity to non-target sequences in NCBI's Nucleotide database were removed. Mock sequencing databases (MSDs) were created using MetaSim. Resulting databases contained 10,000,000 reads at different titers of pathogen abundances. A metasample was considered positive for the presence of a pathogen when a significant number of e-probes were found in a MSD. EDNA detected the target pathogens in metadata with high accuracy, thus, it is a powerful tool for detection of oomycetes from metasamples.

HGT drives evolutionary transitions in Rhodococcus and establishes new pathogenic lineages

J. CHANG, Oregon State University, Botany and Plant Pathology, Corvallis, OR, USA

Rhodococcus are cosmopolitan Gram-positive bacteria with members of specific clades adapted to plants. These bacteria are repeatedly recovered from tissues of various plant species, and are implicated in providing benefits to their hosts. In contrast, Rhodococcus with a virulence plasmid are broad hostrange pathogens that cause growth deformations to over 100 genera of plants, leading to significant losses to the horticultural industry. We used a genomic epidemiological approach to trace the transmission of Rhodococcus in US nurseries. Analysis of chromosomal single nucleotide polymorphisms showed multiple lineages of Rhodococcus can persist on plants. In nurseries, there is evidence for multiple introductions, reservoir populations, and point source outbreaks of the pathogen. Characterization of key isolates confirmed the necessity and sufficiency of the virulence plasmid for pathogenicity and revealed that isolates lacking the plasmid cause beneficial growth to plants. Additionally, results from analyzing the diversity and distribution of virulence plasmids in pathogenic lineages were inconsistent with predicted patterns of pathogen transmission. Evidence suggests that the horizontal acquisition of the virulence plasmid establishes new lineages of pathogens, and can drive the rapid evolutionary transition of the bacteria from being beneficial to being pathogenic.

Modelling the evolution of pathogen virulence in forest pathosystems

C. ROBIN, J. P. Soularue, M. L. Desprez-Loustau, C. Dutech, BIOGECO, INRA, Univ. Bordeaux, Cestas, FRANCE

For the understanding of forest diseases and their management, it is essential to couple epidemiology and evolutionary biology (Desprez-Loustau et al. 2016). Indeed, fungi have evolved diverse abilities to disperse, reproduce and infect different plants or organs. This evolution can occur over short time scales, in response to strong selective pressures exerted by the environment and/or management options. Because sustainable management of forest diseases must be designed at landscape scale and on long timeframes, modelling plays an essential role. We will present an individual-based model used to investigate the effects of management practices on virulence evolution in root rot fungi in pine plantations (Soularue et al. 2017). Based on quantitative genetics theory, this model is genetically and spatially explicit and makes possible to investigate the co-occurring evolutionary and epidemiology processes in practical contexts. Our simulations indicated that shortening the rotation length in pine plantations accelerates both the pathogen virulence and the development of the epidemics. The key influence of the level of genetic variation in emerging fungal pathogen populations was confirmed: it changed quantitatively both the evolutionary change of virulence and the speed of the epidemic. This modelling approach offers the opportunity to study evolutionary and epidemiological dynamics in various species and ecological contexts. It was adapted to the chestnut blight fungus.

Powdery mildews (Erysiphales) in Victorian horticulture: DNA isolation to rediscover an old foe hidden in herbaria

R. SMITH (1), T. Sawbridge (2), R. Mann (3), J. Kaur (3), T. May (4), J. Edwards (3), (1) DEDJTR, Agriculture Victoria, Bundoora, AUSTRALIA; (2) LaTrobe University, Bundoora, AUSTRALIA; (3) AgriBio, Bundoora, AUSTRALIA; (4) Royal Botanic Gardens, Melbourne, South Yarra, AUSTRALIA

Powdery mildews pose a significant threat to the global food trade and can cause massive crop losses throughout agricultural and horticultural industries. Maintaining up to date plant pathogen data is vital for Australian Biosecurity. The Victorian Plant Pathology Herbarium (VPRI) houses over 42,000 plant pathogen specimens. Of these the powdery mildews (Erysiphales) represent $\sim 2700$ specimens, collected from agricultural, horticultural and ornamental host plants. The VPRI plant pathogen database feeds directly into the Australian Plant Pathogen Database (APPD) providing specimen based records of current plant pathogens. Traditionally, morphology and host plant associations were used for the identification of powdery mildews but this has been proven unreliable. The modern approach to fungal taxonomy is to use morphological characters together with genetic analyses. I will present research on the molecular characterisation of VPRI powdery mildew specimens, focusing specifically on horticultural crops of Victoria, Australia. An integral part of this research is the establishment of reliable DNA extraction methods and identification of appropriate gene regions for species identification of preserved powdery mildews specimens. The aim of this project was to test a range of DNA extraction protocols which used varying source material of different ages (fresh, recent -200 years old and ancient) to compare the DNA quantity and quality yielded from the VPRI powdery mildew specimens. We also evaluated fungal gene markers for use in herbarium fungal species identification.

Structure and function of seed microbiomes from 98 plant species P. E. BUSBY (1), M. Ridout (2), E. Barge (1), A. Harding (2), G. Newcombe (2), (1) Oregon State University, Corvallis, OR, USA; (2) University of Idaho, Moscow, ID, USA

Seeds are the organs of generational and genetic renewal and recombination in plants, yet we lack a unified view of the seed microbiome that includes both mutualists and pathogens, and more plants than just crops and a few models. We characterized culturable endophytes from 98 plant species from 39 families (70 seed samples per plant species, pooled from many plants per species), and then tested interactions between seed endophytes and pathogens in Triticum aestivum (winter wheat). Overall, $65.6 \%$ of all surface-sterilized seeds lacked isolates on PDA; $30.6 \%$ were characterized by a single, primary symbiont of varying identity. Less than $4 \%$ of seeds overall hosted two or more culturable endophytes. Despite sampling 16 orders of plants, 
primary symbionts were dominated by fungi in a single order, Pleosporales. In winter wheat, seed endophytes reduced the severity of leaf rust disease caused by Puccinia triticina, with effects stronger in five-year-old seed compared to one-year-old seed. Our results support a simplified seed microbiome that contrasts strongly to the taxonomically diverse vegetative microbiomes of land plants. However, disease modification appears to be function common to endophytes found in seeds, roots, shoots and leaves.

Bacterial endophyte traits in vitro do not predict protection from a fungal pathogen in planta B. Whitaker (1), M. G. BAKKER (2), (1) Indiana University, Bloomington, IN, USA; (2) USDA ARS, Peoria, IL, USA

All plants are colonized by an array of microbiota, with impacts on host function that can span a continuum from beneficial to pathogenic. Increasingly, endophytes (those microbiota that reside inside plant tissues) are seen as potentially useful symbionts for conferring disease suppression or abiotic stress tolerance. Commonly used approaches to identify putatively beneficial symbionts include plate competition assays and co-inoculation on plant substrates in the lab. However, few studies have directly contrasted functional roles inferred from in vitro tests with functional outcomes in planta. This can lead to a failure of candidate biocontrol agents when transferred to more realistic field conditions. Our objective was to test whether traits displayed by bacterial endophytes in vitro would be predictive of disease outcomes in planta. Using two common in vitro assays, we contested bacterial endophytes isolated from wheat plants against Fusarium graminearum, a fungal pathogen that causes Fusarium head blight. A subset of isolates, ranging from weakly to strongly antagonistic in the in vitro assays, were selected for an in planta assay. All assays were performed under variable abiotic conditions to test the climatic resilience of the plant-fungal-bacterial interactions. We found that the degree of pathogen inhibition detected in the two in vitro assays was not predictive of pathogen loads in the in planta assay. This was true across temperature and carbon dioxide conditions chosen to reflect potential future climate change scenarios. Additionally, climatic resilience within the plant-fungal-bacterial interaction varied among bacterial isolates. We suggest that field or in planta trials of the impacts of endophytes on plant performance should include strains that both do and do not show in vitro activity.

Reverse modeling enables estimating yield losses caused by individual and multiple disease injuries L. WILloCQUET (1), I. Félix (2), C. De Vallavieille-Pope (3), S. S. Savary (1), (1) INRA, Castanet Tolosan, FRANCE; (2) Arvalis, Le Subdray, FRANCE; (3) UMR BIOGER AgroParisTech, INRA, Université Paris-Saclay, Thiverval-Grignon, FRANCE

Yield loss analyses are critical to inform tactical and strategic decisions for crop health management. Yield loss measurement requires the quantification of (1) levels of injury caused by disease or pest, (2) actual (injured) yield, and (3) attainable (un-injured) yield. Reverse modelling allows reconstructing an object or a process from limited information combined with a mathematical model. We reconstructed attainable yields and derived yield losses caused by diseases in winter wheat using a simulation model (WHEATPEST) combined with field data generated by a network of experiments across France, where disease injuries and actual yields, but not attainable yields, were measured. The analysis covered 70 [Year x Region x Variety x Crop Management] combinations, from 2004 to 2008. In each combination, three simulation steps were taken in order to model: (1) actual yields, (2) attainable yields, and (3) yield losses associated with individual diseases. Simulated overall yield losses from combined diseases ranged from 0 to $4.2 \mathrm{t}$ $\mathrm{ha}^{-1}$, and averaged $0.80 \mathrm{tha}^{-1}$. Septoria tritici blotch caused the highest mean yield loss of $0.66 \mathrm{t} \mathrm{ha}^{-1}$. Other diseases causing losses, in decreasing order, were FHB, leaf rust, stripe rust, and powdery mildew. The results highlight the contribution of varietal improvement to agricultural sustainability. Reverse modelling can be applied to other crops and diseases or pests, in order to estimate individual and combined diseases yield losses.

Planted forest health: The need for a global strategy

M. WINGFIELD, B. D. Wingfield, B. Slippers, Forestry and Agricultural Biotechnology Institute (FABI), University of Pretoria, Pretoria, SOUTH AFRICA

Planted forests, especially those of non-native tree species have been dramatically expanded in extent during the course of the past 100 years. This has occurred to meet a growing need for timber products including paper, packaging and most recently wood-based chemicals. Non-native trees separated from their natural enemies are typically highly productive in the early stages of establishment. This situation changes as pests (including insects and pathogens) are accidentally introduced into the new plantation environments. There are also growing numbers of examples where pathogens native to these new environments undergo host jumps to infect the non-native trees or where pathogens have changed genetically to infect trees previously not susceptible to them. Some of these novel pathogens emerging after host jumps are now also causing devastating epidemics on native trees in forests and woody ecosystems. In addition, a bridgehead effect, where invasive pest populations serve as the source for further introductions into new areas, has emerged as a major driving force in the increased rate of emergence of new pest threats. It is increasingly clear that many factors relating to the patterns and processes underlying pest epidemics in planted forests are poorly understood and that their importance has been underestimated. A global strategy, including enhanced research efforts, international collaboration and significantly improved quarantine processes will be required to deal with this problem. This is not only to ensure the sustainability of planted forests, but also the health of crucially valuable natural forest ecosystems.

Two new Ceratocystis species cause the serious and devastating rapid 'ōhi`a death (ROD) on native Metrosideros polymorpha in Hawai i I. BARNES (1), M. J. Wingfield (1), T. Harrington (2), L. M. Keith (3), (1) Forestry and Agricultural Biotechnology Institute (FABI), University of Pretoria, Pretoria, SOUTH AFRICA; (2) Iowa State University, Ames, IA, USA; (3) USDA-ARS, Hilo, HI, USA

New reports of Ceratocystis species, causing disease outbreaks on tree species globally, have increased in recent years. Some of the most aggressive Ceratocystis spp. recently emerging as serious threats to trees include C. manginecans on plantation-grown Acacia mangium in South East Asia, and C. platani killing Platanus orientalis in Europe. Recently, a serious new disease known as rapid 'ōhi a death (ROD), has emerged on native Metrosideros polymorpha trees in Hawai $i$ and has resulted in the death of hundreds of thousands of trees. Investigations into the causal agent of the disease revealed the presence of two novel Ceratocystis species. Based on a combination of the morphological, phylogenetic and biological species concepts, these fungi have been described as Ceratocystis lukuohia residing in the Latin American Clade, and Ceratocystis huliohia residing in the Asian-Australian Clade of Ceratocystis senu lato. Ceratocystis lukuohia is most closely related to isolates from Syngonium and Xanthosoma from the Caribbean and other regions (including Hawai i), and C. platani, which is native to eastern USA. Ceratocystis huliohia is most closely related to the newly described species of $C$. uchidae, C. changhui and C. cercfabiensis, which are considered to be native to Asia. Screening of the isolates with microsatellite markers suggests that both species are clonal in Hawai'i and are likely recently introduced pathogens. This disease threatens a native tree species which is of ecological, cultural and biological significance to Hawai $i$. The serious nature of the disease highlights the need for an integrative management approach to prevent the spread of both pathogens, which are currently restricted to the Big Island.

Suppressive Soils: New Paradigms for an Old Story?

L. S. THOMASHOW (1), D. Mavrodi PhD (2), O. V. Mavrodi PhD (3), L. Elbourne (4), S. Tetu (5), M. LeTourneau (6), R. Bonsall (7), J. Parejko (8), M. Yang (9), I. T. Paulsen (10), D. M. Weller (11), (1) USDA-ARS, Pullman, WA, USA; (2) Univ of Southern Mississippi, Hattiesburg, MS, USA; (3) 
University of Southern Mississippi, Hattiesburg, MS, USA; (4) Department of Chemistry and Biomolecular Sciences, Sydney, NSW, AUSTRALIA; (5) Chemistry and Biomolecular Sciences, Sydney NSW, AUSTRALIA; (6) Washington State University, Pullman, WA, USA; (7) Department of Plant Pathology, Pullman, WA, USA; (8) Department of Chemistry and Molecular Biology, Saint Peter, MN, USA; (9) Northwest A\&F University, Yangling, CHINA; (10) Department of Chemistry and Biomolecular Sciences, Sydney NSW, AUSTRALIA; (11) USDA-ARS Wheat Health, Genetics and Quality Research Unit, Pullman, WA, USA

Long known as biocontrol agents, phenazine-1-carboxylic acid (PCA)-producing pseudomonads are widely distributed across dryland cereal cropping systems of the Pacific Northwest, USA. We investigated the effect of soil moisture on the population levels, diversity, and composition of rhizosphere microbial communities, and the mechanisms driving accumulation of PCA producers in soils. A field study conducted for 3 consecutive years in adjacent irrigated and dryland plots at a long-term wheat monoculture site revealed the magnitude of changes in the wheat rhizosphere microbiome in response to soil moisture and temperature, and shed light on the impact of environmental factors on indigenous bacterial communities and phenazine production in situ. Related studies in mesocosms revealed that PCA biosynthesis genes were expressed under both dryland and irrigated conditions, that PCA persisted in soils with a half-life of 3 to 4 days, and that PCA-producing bacteria on dryland roots were associated with more robust biofilms than were colonies of an isogenic PCA nonproducer. PCA also influenced the turnover of biomass from ${ }^{15} \mathrm{~N}$-labeled bacteria, including the transfer of ${ }^{15} \mathrm{~N}$ from bacteria to roots. Collectively, our results indicate that PCA production is an adaptation to water stress that promotes the formation and development of rootassociated biofilms, potentially influencing the availability of bacterial $\mathrm{N}$ to crops and the formation of soil organic matter.

Insights into biological and molecular underpinnings of how post-plant nematicides suppress Meloidoygne incognita C. WRAM (1,2), A. Peetz (3), I. A. Zasada (4), (1) Oregon State University, Corvallis, OR, USA; (2) USDA ARS-HCRU, Corvallis, OR, USA; (3) USDA ARS HCRU, Corvallis, OR, USA; (4) USDA ARS, Corvallis, OR, USA

Meloidogyne species, root-knot nematodes, are one of the most important groups of plant-parasitic nematodes globally due to their ability to infect and damage almost all cultivated plants. Although widespread and economically important, there are few control measures to combat these pests. Recently several new nematicides have been discovered that can be used to manage nematodes post-plant. To gain a deeper understanding of the mechanisms of these new chemistries and use this understanding to maximize efficacy and minimize the development of resistance, we characterized gene expression in Meloidogyne incognita treated with sub-lethal doses of common four post-plant nematicides. Second-stage juveniles (J2) were exposed to a 24-hour sublethal dose of four post-plant nematicides, which resulted in $50 \%$ mortality $\left(\mathrm{LD}_{50}\right)$, after which RNA was extracted and gene expression determined using RNAseq. Post-plant nematicides used in this study were oxamyl, fluopyram, fluensulfone, and fluazaindolizine, which represent new and old nematicides, encompass various modes of action, and represent a range of $\mathrm{LD}_{50}$ concentrations. Twenty-four hour $\mathrm{LD}_{50}$ concentrations for oxamyl, fluopyram, fluensulfone, and fluazaindolizine were $100 \mathrm{ppm}, 1 \mathrm{ppm}, 95 \mathrm{ppm}$, and $230 \mathrm{ppm}$, respectively. In water replacement experiments and mobility assays, exposure to sublethal doses of fluazaindolizine and fluensulfone resulted in death of $M$. incognita, while the nematode recovered from sublethal doses of oxamyl and fluopyram. Over 2,500 genes were significantly differentially expressed in Meloidogyne incognita $\mathrm{J}_{2}$ exposed to $\mathrm{LD}_{50}$ concentrations, with a 16 -fold increase in expression of iron binding proteins and oxioreductases.

\section{Improving CRISPR/Cas9 tools for precise genome editing of host plants and fungal pathogens} Y. YANG, Pennsylvania State University, University Park, PA, USA

The bacterial cluster regularly interspaced short palindromic repeats (CRISPR)/CRISPR-associated protein 9 nuclease (Cas9) system is a powerful and versatile tool for genome editing. Using the rice-Magnaporthe oryzae pathosystem as a model, we have developed and improved bioinformatics and molecular tools for CRISPR/Cas9-enabled genome editing and targeted mutagenesis in the host plant and the fungal pathogen. To improve DNA editing specificity, genome-wide prediction of specific guide RNA (gRNA) spacers was performed for the rice blast fungus and eight plant species. The engineered gRNA/Cas9 system were shown to induce specific cleavages and targeted mutations at the desired genomic sites in rice and $M$. oryzae. In addition, the polycistronic tRNA-gRNA editing strategy was developed to facilitate multiplex genome editing, DNA fragment deletion, and other more sophisticated applications in plants, fungi and other organisms. With improved bioinformatics and molecular tools, the CRISPR/Cas9 system is being demonstrated to have broad applications in functional genomic analysis of host-pathogen interactions and precision breeding of crop ideotypes with enhanced disease resistance and other beneficial traits.

\section{Diversity among S morphology fungi in Aspergillus section Flavi from North America}

\section{P. SINGH (1), P. J. Cotty (2), (1) University of Arizona, Tucson, AZ, USA; (2) USDA-ARS, University of Arizona, Tucson, AZ, USA}

Aflatoxins are hepatocarcinogens that contaminate a wide range of crops in warm regions, and are produced by several species in Aspergillus section Flavi. Several phylogenetically distinct members of section Flavi have S morphology (small sclerotia) and produce high levels of aflatoxins (B or B and G). Fungi with S morphology have been implicated as causal agents of contamination in both the United States (US) and Africa. Several S morphology taxa remain unnamed. One of these, the Lethal Aflatoxicosis Fungus (LAF), has been associated with over 100 deaths from aflatoxicosis in Kenya. Genetic diversity of S morphology fungi in North America (NA) is poorly understood. The current study characterized fungi with $\mathrm{S}$ morphology ( $\mathrm{n}=504)$ collected from 2002 to 2015 from soil and maize, collected from across the US and Mexico. Phylogenetic analyses of partial gene sequence for aflatoxin pathway transcription factor $(a f l R, 1.8 \mathrm{~kb})$ resolved fungal isolates into four distinct clades. Aspergillus flavus $\mathrm{S}$ strain was predominant among $\mathrm{S}$ morphology fungi from NA ( $96 \%)$ followed by $A$. texensis $(2.2 \%)$, LAF $(0.6 \%)$ and a novel clade of B aflatoxin producers sister to $A$. flavus $(1.2 \%)$. The $\mathrm{S}$ strain of $A$. flavus was found to be highly diverse at 17 microsatellite loci with 292 haplotypes from 484 isolates and 5 to 27 alleles per locus. Occurrence of distinct species and haplotypes of aflatoxin-producing fungi provide a new perspective on the etiology of aflatoxin contamination in NA.

\section{Moving Forward with the Identification of Fungi Using Sequence Based Techniques} A. PORRAS-ALFARO, Western Illinios University, Macomb, IL, USA

With the advent of large sequencing efforts of environmental samples, fungal biologists have been confronted with new and challenging opportunities for the discovery and characterization of novel species of fungi. The characterization of vast numbers of endophytic fungi in plants has also created new challenges due to the bias in curated databases toward plant pathogenic fungi and the limiting tools to distinguish specific ecological roles of endophytes. New platforms to facilitate the rapid identification of fungi are being developed to address these new challenges. However, standardized practices and mechanisms to integrate technologies are lacking. In this talk, I will discuss specific initiatives to take advantage of new technologies that will advance the discovery of fungi, characterize their local, regional and global distribution, inform cultured-based efforts for the discovery and characterization of species, and integrate traditional as well as modern techniques to provide a more accurate characterization of fungal species. 
GC-MS metabolic pathways associated to the different stages of banana black sigatoka disease (BSD)

M. G. MARIDUENA-ZAVALA (1), L. De Weerdt (2), M. J. Molina (3), A. Quevedo (3), D. Ochoa (1), J. Cevallos-Cevallos (1), (1) Escuela Superior Politecnica del Litoral, ESPOL, Guayaquil, ECUADOR; (2) Ghent University, Brussels, BELGIUM; (3) Escuela Superior Politecnica del Litoral, Guayaquil, ECUADOR

Banana (Musa acuminata) production is a significant source of income in many countries and black sigatoka (BSD) is one of the most devastating banana diseases worldwide. However, the metabolic changes occurring in bananas at the different stages of BSD are poorly understood. This work aimed to evaluate the metabolite profile of plants infected with BSD using a GC-MS. Four months old banana plants were inoculated with $1 \times 10^{5}$ cell $/ \mathrm{mL}$ of Pseudocercospora fijiensis - the causal agent of BSD - ground mycelia and kept in a greenhouse. The disease progress was evaluated during six months and leaves with different symptomatic stages were sampled. Metabolites were extracted from symptomatic leaves using methanol, chloroform and water, followed by a derivatization using MSTFA (N-methyl-N- trimethylsilyl trifluoracetamide) and detection using GC-MS. Multivariate analysis revealed distinct metabolite profile of infected plants at different stages of BSD when compared with healthy plants. Various metabolites including Propanoic acid, Dotriocontano, L-Threonic acid, Ribonic acid, $\beta$-D-Galactopyranoside, Xylonic acid, Glucaric acid, Quercetin, Isochiapin B, Hexadecanoic acid, Nonadecanoic acid, D-(-)-Fructofuranose, D-(-)-Tagatofuranose, 4 D-(-)-Ribofuranose were significantly downregulated in infected plants while Xylonic acid, Octadecanoic acid, Glyceryl-glycoside, Glutamic acid, $\beta$-D-Allopyranose, Acetopyruvic acid, $\beta$-D-(-)-Ribopyranose, 2-Keto-I-gluconic acid, 3- $\alpha$ Mannobiose were significantly upregulated in the presence of BSD. Pathways associated to the disease progression include Flavone and flavonol, unsaturated fatty acids, Cutin, Phenylpropanoid, Flavonoid, Stilbenoid, diarylheptanoid and gingerol. Biosynthesis. This is the first report of metabolic changes occurring during BSD infection.

Banana Elephantiasis Disease: An emerging disease for Latin America

F. ALIAGA, University of Buenos Aires, Cuidad Autonoma de Buenos Aires, ARGENTINA

Bananas (Musa acuminata) and plantains (Musa balbisiana) are an important food source to families and farmers dedicated to their production in the tropics and subtropics, vital to quality of life and the agricultural economy of the region. Banana Elephantiasis Disease (BED) has unknown etiology, but has been reported in Latin American countries where it causes many economic losses. BED is currently an emerging disease which reduces the commercial value of their bunches and limits their production in Colombia and Peru. BED is exhibited at the pseudostem-rhizome junction with longitudinal and transverse ruptures at the pseudostem base, causing the plant to fall down. In the districts of San Luis de Shuaro and Pichanaki of the department of Junín of Peru, this disease affects the cultivars of Seda (Group AAA), William (Group AAA), Morado (Group AAA), Isla (Group AAB) and Palillo (Group AAB). According to a survey conducted during 2015 and 2017, this disease reduces the yields of Gros Michel (Group AAA) and Dominico Hartón (Group AAB) anywhere from $9 \%$ to $71.6 \%$ in the municipalities of Ulloa and Alcala in the department of Valle del Cauca in Colombia. The random distribution of the disease observed in the field suggests the possible participation of insect vectors, secondary hosts, and the use of infected planting material. DNA was extracted from symptomatic and asymptomatic plant rhizomes by each farm to amplify the V4 region of the $16 S$ gene with 515F/806R primers in the HiSeq Illumina platform. Plants grown in vitro were used as negative controls. 12 OTUs of Candidatus Phytoplasma were found only in symptomatic plants. BLAST analysis showed a 99\% sequence identity (MF977376-MF977387) with Candidatus Phytoplasma. This research demonstrates that Candidatus Phytoplasma is an organism associated with Banana Elephantiasis Disease.

The strategic role of dissemination and communication in raising awareness on new IPM practical solutions. The EMPHASIS project A. BERTIN (1), L. Vivani (2), A. Masino (3), (1) Spin-To srl, Torino, ITALY; (2) Moverim Consulting sprl, Bruxelles, BELGIUM; (3) Agroinnova University of Torino, Grugliasco, Torino, ITALY

EMPHASIS is a project funded by European Union's Horizon 2020 programme with 22 partners from 10 different countries. EMPHASIS aims to fight native and alien pest threats throughout a multi-actor approach in order to predict, prevent and protect agriculture and forestry system. This means that EMPHASIS put food security and plant health in the spotlight. The dissemination and communication of scientific messages isn't an easy task, as the figures generated in labs are difficult to translate into popular formats. We know that that the first communication tool for researchers are scientific papers, but EMPHASIS has to facilitate the transfer of scientific results to enterprises, policy makers, farmers, associations, and to a wide public at large. That's why, within a dedicated workpackage, traditional and innovative communication instruments work together to engage different stakeholders: from media relations to social media; from a virtual collaborative platform, webinars and summer schools to new outreach formats such as stage shows in theatre. So far these activities have generated 76 articles on the public press, more than 550.000 views on Twitter and 25.000 on Facebook with a potential global reach of more than 170.000 people, building a growing community focussed on Emphasis' topics. The combination of awareness raising and capacity building aspects allows to use multiple channels to disseminate the project findings and to stimulate new networking linkages.

Role of $h s p 70$ and calreticulin gene on temperature adapatation of Blumeria graminis f. sp. tritici Z. Wang, M. Zhang, J. FAN, Y. Zhou, Institute of Plant Protection, CAAS, Beijing, CHINA

Wheat powdery mildew, caused by Blumeria graminis f. sp. tritici (Bgt), is one of the most important wheat diseases worldwide. Temperature is considered to be an important climate factor affected disease epidemics and pathogen populations. As the climate warm up, over-wintering range of this disease is increased. Temperature tolerant strains have been isolated in the fields in China. To understand the basis of tolerance to temperature of Bgt, the expression level of heat shock protein $70(h s p 70)$ and calreticulin gene in temperature-tolerant and -sensitive isolates were investigated. The relative expression of $h s p 70$ and calreticulin were increased in both phenotypes after heat-treat with $26^{\circ} \mathrm{C}$ before inoculating on wheat seedlings compared with treatment at $18^{\circ} \mathrm{C}$, the optimum growth temperature for. In addition, the relative expression of $h s p 70$ and calreticulin is higher in temperature-tolerant strains in comparison with sensitive strains. These data suggested that hsp70 and calreticulin played important roles on adaptability to high temperature in Bgt.

Reinventing the wheel or driving the science? International diagnostics: Capacity and coordination M. AREVALO (1), C. Lapaire Harmon (2), (1) Agroexpertos, Guatemala City, GUATEMALA; (2) University of Florida, Gainesville, FL, USA

Trade in agronomic and horticultural products is a known pathway for hitchhiking pathogens and pests. Sources and recipients of those products must have the infrastructure to detect and accurately diagnose diseases promptly and to protect against or proactively manage those diseases. Early detection allows for the possibility of eradication or containment, while accurate diagnosis is paramount for recommendation of effective action. Training in these vital areas, encouragement of communication between countries, and science-based action, have been supported and disseminated by many agencies, countries, and organizations over the decades in the global fight against agricultural pests. Groups such as CABI, USAID, USDA-FAS, the National and International Plant Diagnostic Networks, the National Plant Biosecurity Diagnostic Network, and others have all had their share of success and failure to provide a continuum of training and diagnostic capacity in-country and around the world. To date, piecemeal funding, duplicative programming, and lack 
of coordination on a global scale have hindered the sustainability and impact of these programs. We will discuss examples of successful programs, pose funding priorities for delegates to consider, and describe possible solutions to some of the training and diagnostics dilemmas that derail once-successful projects.

Effect of irrigation on the severity of charcoal rot and yield on selected drought tolerant soybean genotypes

A. MENGISTU (1), J. Ray (2), J. R. Smith (2), P. Arelli (1), N. Bellaloui (2), P. Chen (3), S. J. Grover (4), D. Boykin (2), (1) USDA ARS, Jackson, TN, USA; (2) USDA-ARS, Stoneville, MS, USA; (3) Univ. of Missouri, Portageville, MO, USA; (4) Univ of Missouri, Portageville, MO, USA

Charcoal rot, (caused by Macrophomina phaseolina), has historically resulted in severe yield losses of soybean [Glycine max (L.) Merr.] in the U. S. and around the world. Identification of resistance and yield measurements could be complicated because of the confounding effect of varying drought stress levels from year to year. The objectives were to determine the severity of charcoal rot (CR) in the putative drought tolerant genotypes and to determine the over-all effect of $\mathrm{CR}$ on yield in irrigated and non-irrigated environments. Colony Forming Units (CFUs) at reproductive stage R7 were used to assess disease severity and classify each genotype's response to CR. A stress tolerance index (STI) was used to determine the relative impact of soil moisture stress (i.e. no irrigation) on the 13 genotypes. Over the three years $(2011,2012$ and 2013) in both irrigated and non-irrigated environments, five genotypes were consistently rated as moderately resistant to CR and three genotypes were consistently rated as susceptible to CR, whereas the responses of the remaining five genotype's varied. Averaged over the three years, there was a wide range ( 0.36 to 1.09$)$ of STI values among genotypes. Even though there was a consistent trend, there was a very weak relationship between STI and CFU's at R7. Regression analysis indicated that as CFUs at R7 increased, seed yield decreased, although the relationship was not significant in every year and irrigation environment. Nonetheless, across all years and irrigation environments, a pooled (global) slope indicated a yield loss of $11.5 \mathrm{~kg} \mathrm{ha}^{-1}$ for every 1,000 CFUs at R7. These data indicated that as CFUs at R7 increased, seed yield decreased in both irrigated and non-irrigated environments suggesting that, it may be necessary to select for resistance to both traits using environments where both soil moisture stress and CR are high.

Manipulating the grapevine microbiome for novel control strategies of Pierce's Disease E. DEYETT, P. E. Rolshausen, University of California, Riverside, Riverside, CA, USA

Host-microbe-pathogen interactions are highly dynamic and are poorly understood especially in the context of agricultural cropping systems. However, advances in sequencing technologies have revealed how vitally important microbial diversity and communities are for plant health. By using metagenomic tools, microbes can be identified as being antagonistic to pathogens; thus benefitting the host. This work builds upon that knowledge by utilizing the microbial communities associated with grapevine to find novel microbial alternative management strategies for Xylella fastidiosa $(X f)$, the causative agent of Pierce's Disease (PD). We hypothesized that the vines' microbial composition influences $X f$ titer and colonization, affecting disease outcome. The aim of this project is to study microbial community temporal and spatial dynamics in PD-symptomatic and asymptomatic bicompartments of grapevines. The overarching goal is to identify biocontrol agents and anti-Xylella bioactive natural molecules of microbial origin for preventative and curative control strategies for PD, respectively. Metagenomic tools and culture-independent methods were utilized to reveal potential beneficial organisms that mitigated PD. The discovery of novel natural microbes to combat pathogens using "Omics" technologies could be an alternative approach to limit the risks of pesticide resistance, increase crop productivity, and support of sustainable agriculture.

Diversity of the rice pathogenic bacterium Burkholderia glumae in virulence, regulatory system, and genome structure associated with genomic islands

J. H. HAM (1), T. De Paula Lelis (1), H. H. Lee (2), Y. S. Seo (2), (1) Louisiana State University, Baton Rouge, LA, USA; (2) Pusan National University, Busan, KOREA

Burkholderia glumae is a gram-negative plant pathogenic bacterium causing bacterial panicle blight in rice. The phytotoxin toxoflavin, along with its derivatives, is a major virulence factor of this pathogen, and the quorum-sensing mediated by the luxI/luxR homologs tofI/tofR plays a pivotal role in the regulation of bacterial pathogenesis. Genetic studies of two virulent strains 336gr-1 and 411 gr-6 showing contrasting phenotypes in pigmentation revealed that the regulatory system for bacterial pathogenesis is different between these two strains. Remarkably, 411 gr- 6 retained pathogenic functions in the absence of the tofI/tofR quorum-sensing system, while 336gr-1 completely lost its pathogenic function in the same genetic background. We recently completed whole genome sequencing of these two virulent strains and a naturally avirulent strain 957856-41-c to investigate the genomic structure determining the phenotypic variations of these strains. Comparative analysis of these three genome sequences and two reference genome sequences in the NCBI database (one for the virulent strain BGR1 and the other for the avirulent strain ATCC33617) revealed significant variations in the genome structure across the five strains of B. glumae. These variations were mainly associated with the presence of multiple strain-specific genomic islands (GIs). The features of the strain-specific GIs associated with the phenotypic variations of $B$. glumae strains will be discussed.

Dickeya fangzhongdai causing soft rot of Phalaenopsis orchids and bacteriophage biocontrol options Š. ALIČ (1,2), T. Naglič (1,3), F. Van Gijsegem (4), J. Pédron (4), M. Tušek Žnidarič (1), M. Peterka (3), M. Ravnikar (1), T. Dreo (1), (1) National Institute of Biology, Ljubljana, SLOVENIA; (2) Jožef Stefan International Postgraduate School, Ljubljana, SLOVENIA; (3) COBIK, Ajdovscina, SLOVENIA; (4) iEES Paris, Paris, FRANCE

Bacterial soft rots represent a serious limitation to the production of ornamental and others horticultural plants world-wide e.g. potatoes, tomatoes and orchids, with no effective chemical control. The aim of our study was to isolate and characterize bacteria causing soft rot of orchids (Phalaenopsis sp.) and to investigate bacteriophages as biocontrol agents for their management. Bacteria isolated from diseased tissues of Phalaenopsis orchids from a commercial production site were identified as Dickeya spp. However, none of the performed tests was able to resolve classification to a species level. Therefore, genomes of two representative Dickeya sp. isolates were sequenced. As part of a comparative genomic study of genus Dickeya, average nucleotide analysis, DNA-DNA hybridisation and multilocus sequence analysis on six housekeeping genes were performed, placing the newly sequenced Dickeya to a recently described D. fanhzhongdai. To confirm the observed taxonomic position of our Dickeya isolates the phenotypic analysis was performed. Aggressiveness of the bacteria was tested phenotypically on the model plants, Belgian chicory and potato tubers, exhibiting extreme maceration. Beside, inoculation of the viable potato plants was performed, showing the bacteria ability to survive and spread through the plant tissue. On the other hand, possible approaches for the Dickeya biocontrol were studied. Bacteriophages effective against new Dickeya strains were isolated from the diseased orchids and sewage water. Three different groups of bacteriophages from Caudovirales family were identified. All bacteriophages were lytic and specific for the genus Dickeya, therefore represented suitable candidates for phage biocontrol. 
Asia is home to 800 million of the world's 1.1 billion poor and accounts for $90 \%$ of global rice consumption. Only $7 \%$ of the annual global rice production is traded internationally by a few exporting countries. Local variation in production may then have large impacts on trade and price. Reduction and variation in rice production is partly attributed to yield losses caused by diseases and pests. However, there have been limited efforts prior to the late 1980 s to systematically quantify the impact of rice diseases and pests compared with other cereals. Several reports based on farmers' field surveys and experiments conducted since 1987 have documented the importance of diseases, insect pests, and weeds of lowland rice in Asia, the linkages between production situations and injury profiles, and the potential of improved crop health management to sustainably generate yield gains. Recent surveys of farmers' fields conducted in tropical Asia showed increasing adoption of specific cropping practices and the emergence of new production situations. These production situations resulted in the emergence of new diseases and disease profiles, along with new crop loss patterns. Results highlight the need to consider the variation and dynamics in production situations to understand the impact of diseases and develop pest management strategies in tropical Asia.

\section{Consequences of plant disease introductions: From crop loss mitigation to environmental impact} M. J. JEGER, Imperial College London, Ascot, UNITED KINGDOM

Introductions of plant pathogens to new regions, countries and continents have a range of consequences, especially where disease outbreaks have the potential to develop into major epidemics in time and space. The consequences are multidimensional, even without taking into account socio-economic and cultural considerations. The consequences include: crop losses in terms of yield, quality, and production area; the need for additional control measures, including long-term increases in the use of plant protection products; impact on amenity trees, woodlands and forested areas; environmental and other undesired impacts of control measures; and impacts on native plants, biodiversity and ecosystem services. New quantitative methods are being developed to assess the risks of introduction (entry and establishment) and subsequent spread of invasive plant pathogens in the European Union. The most problematic area and challenge is to assess quantitatively the consequences of introduction and spread of a plant pathogen, especially where there are crop loss, cropping area and environmental impacts to be considered. The challenges faced in assessing these impacts will be illustrated for the following examples: Diaporthe vaccinii, Cryphonectria parasitica, Ditylenchus destructor, and the 'Flavescence doree' phytoplasma. These examples cover the range of plant pathogen taxa, host types, and the relative importance attached the dimensions of impact considered.

\section{Development of a Next-Generation Sequencing screening method for exotic forest pathogens from fungal spores in air and occurring on insect} vectors

E. D. TREMBLAY (1,2), J. Berube (3), T. Kimoto (4), C. Lemieux (5), L. Bernier (6,7), G. J. Bilodeau (1), (1) Canadian Food Inspection Agency, Ottawa, ON, CANADA; (2) Université Laval, Québec, QC, CANADA; (3) Canadian Forest Service, Ste Foy, QC, CANADA; (4) Canadian Food Inspection Agency, Burnaby, BC, CANADA; (5) Universite Laval Institut de Biologie Integrative et des systèmes, Quebec, QC, CANADA; (6) Institut de Biologie Intégrative des Systèmes (IBIS), Université Laval, Québec, QC, CANADA; (7) Université Laval, Centre d’Étude de la Forêt (CEF), Quebec, QC, CANADA

Anthropogenic practices, namely agriculture and horticulture, are responsible for the dispersal of phytopathogenic fungal spores in the environment. While the volume of international trading increases yearly, so does the introduction and establishment of invasive and exotic fungal phytopathogens. Similarly, wood-boring insects threaten forests by facilitating spore movement to host trees. To protect forest resources, early detection of exotic fungi is the key in managing invasive species. Our new approach uses next-generation sequencing (NGS) and metagenomics for rapid and high-throughput species screening near high-risk areas in Canada. Based on a list of several regulated invasive species, we screened 398 air and insect trap samples from 2013 to 2015 . We designed barcoded PCR primers and produced tagged amplicons by amplifying the ITS1 from fungi and oomycetes. We amplified the ATP9-NAD9 region from oomycetes detected to increase Phytophthora spp. species resolution. In parallel to the 15 NGS runs ( 45 million reads) performed on the Ion Torrent platform, we validated the results using species-specific qPCR assays and observed similar results comparing both techniques. We detected economically important pine pathogens Heterobasidion annosum s.s. and H. abietinum/parviporum and several Phytophthora species, which highlights the benefits of our powerful approach to increase surveying capacity to screen for phytopathogens.

Monitoring wheat powdery mildew using Burkard volumetric spore sampler and unmanned aerial photography W. Liu (1), X. Cao (2), X. Xu (3), J. Fan (1), Z. Wang (1), Z. Yan (1), Y. Luo (4), J. West (5), Y. ZHOU PHD (1), (1) Institute of Plant Protection, CAAS, Beijing, CHINA; (2) Environment and Plant Protection Institute, CATAS, Haikou, CHINA; (3) NIAB East Malling Research, Kent, UNITED KINGDOM; (4) University of California - Davis, Parlier, CA, USA; (5) Rothamsted Research, Hertfordshire, UNITED KINGDOM

Wheat powdery mildew caused by Blumeria graminis f. sp. tritici $(B g t)$ is one of the most important wheat diseases in China. Burkard volumetric spore samplers (2012-2015) and unmanned aerial vehicles (UAV) (2010-2014) were used to monitor wheat powdery mildew. The results of monitoring conidia of Bgt in the air of wheat fields with a Burkard volumetric spore sampler demonstrated that the conidia concentration of Bgt within the canopy was positively correlated with that above the canopy and was significantly higher than that above canopy. Time series analysis showed that conidia concentration was fitted with simple ARIMA $(1,1,0)$ models, and models for prediction of disease severity were constructed with inoculum only, and with both inoculum and weather variables, respectively. A model with inoculum only has more universal applicability to predicting disease severity in comparison with the model based on both inoculum and weather variables. The results of high-resolution aerial imaging with UAV showed that parameter $\operatorname{lgR}$ was consistently correlated positively with mildew severity and wheat grain yield for all combinations of flight altitude and year. Fitting the data with random coefficient regression models showed, however, that the exact relationship of lgR with disease severity and grain yield varied greatly with year, and to a lesser extent with flight altitude within the same year. The present results raised an important question of the consistency of using remote imaging information to estimate disease severity and grain yield. Further research is needed to understand the nature of the variability in the relationship of remote imaging information with disease and grain yield among years.

Apoplastic Venom Allergen-like Proteins of Plant Parasitic Nematodes Modulate the Activation of Damage Triggered Immunity by Cell Surface Receptors

J. L. LOZANO-TORRES (1), R. H. P. Wilbers (1), S. Warmerdam (1), K. Varossieau (1), J. J. Willig (1), C. C. van Schaik (1), O. A. Asojo (2), R. Darwiche (3), R. Schneiter (3), C. Drurey (4), R. M. Maizels (4), A. Goverse (1), A. Schots (1), G. Smant (1), (1) Laboratory of Nematology, Wageningen University, Wageningen, NETHERLANDS; (2) National School of Tropical Medicine, Baylor College of Medicine, Houston, TX, USA; 
(3) Division of Biochemistry, Department of Biology, University of Fribourg, Fribourg, SWITZERLAND; (4) Wellcome Centre for Molecular Parasitology, University of Glasgow, Glasgow, UNITED KINGDOM

Despite causing considerable damage to host tissue, nematodes establish persistent infections in both animals and plants. An elaborate repertoire of nematode effectors modulate damage-triggered immune responses. However, the mode of action of most of nematode immunomodulatory compounds is unknown. We discovered that the nematode effectors venom allergen-like proteins (VAPs) selectively suppress host immunity during the onset of parasitism. VAPs are uniquely conserved in secretions of all animal- and plant-parasitic nematodes, but their role in parasitism has remained elusive. Knocking-down the expression of Gr-VAP1 hampered the infectivity of Globodera rostochiensis. By contrast, heterologous expression of Gr-VAP1 and VAPs from Heterodera schachtii in plants resulted in the loss of basal immunity to multiple pathogens. Surprisingly, VAPs only affect the defense responses mediated by surface-localized immune receptors. The modulation of basal immunity by ectopic VAPs involves extracellular protease-based host defenses and jasmonic acid responses. Crystal structures of VAPs revealed lipid binding motifs which can bind palmitate and sterol both in vitro and in vivo. The delivery of VAPs into host tissue coincides with large modifications in the extracellular matrices by migratory nematodes. We, therefore, conclude that parasitic nematodes most likely utilize VAPs to suppress the activation of defenses by immunogenic breakdown products in damaged host tissue.

Exploiting bacterial genomics to develop tools for effective pathogen monitoring in rice

R. OLIVA (1), C. M. Vera Cruz (2), (1) International Rice Research Institute, Los Banos, PHILIPPINES; (2) International Rice Research Institute, Los Baños, Laguna, PHILIPPINES

The recurrent emergence of highly aggressive clones of plant pathogens in agricultural ecosystems represents an important threat to food security. Bacterial blight, caused by Xanthomonas oryzae pv. oryzae (Xoo), is the most important bacterial disease of rice. At the population level, Xoo is usually composed of a number of phenotypic groups (races), which show R gene-specific interactions. Estimating the race composition of Xoo in the rice paddy is critical to design sustainable management strategies. In this work, we used bacterial genomics to develop DNA markers for effective surveillance of Xoo races in the Philippines archipelago. We first identified the evolutionary forces that shape contemporary groups during the last 40 years of Xoo outbreaks. Through comparative genomics on 100 highly informative strains, we identified six Xoo populations, which diverge before the colonization of the islands. The patterns of positive selection, recombination, and diversification of effector genes suggest that each population experienced a distinct adaptation process that led to the creation of modern races. Using this information, we developed single nucleotide polymorphism (SNP) markers that allow us to detect the pathogen groups in the rice leaf. We validated the robustness of SNP markers for three cropping seasons using a trapping system based on near-isogenic lines carrying different R genes, and demonstrated that real-time surveillance of Xoo is possible. In this scenario, we are proposing an interactive platform, called Pathotracker, which integrates early-season race diagnostics, modeling of weather patterns, and disease resistance profiles to accurately predict which variety should be used in the following season. The platform will allow us to manage bacterial blight epidemics in real-time and to define breeding priorities for the region.

50 Years with the International Society for Plant Pathology - A Magical Mystery Tour

C. J. DELP (1), G. I. Johnson (2), (1) Retired, Tampa, FL, USA; (2) C/- Horticulture for Development, Jamison Centre, AUSTRALIA

Together we journey from contentious beginnings in the 1960's and an exemplary first Congress in London (1968) to congresses in Minneapolis, Muchen, Melbourne, Kyoto, Montreal, Edinburgh, Christchurch, Torino, Beijing, and now, Boston. In 1968, we heard first reports of in-vitro culture of an obligate parasite; the first really systemic fungicide; and a mycoplasma as a plant-pathogen. Now it's the era of microbiomics, tools for research on the microbiome and phytobiome, to enhance resistance, optimise biological balance and reduce disease loss. Over 50 years, emphasis on people focused science delivery has also grown, with ISPP becoming a unique professional society underpinned by context, community and collaboration. The ISPP promotes worldwide development of plant pathology. It's a partnership with 60 national and regional societies and 20 subject matter committees, connected by the ISPP Newsletter, website, and Member Directory. Special honors include the awards of ISPP Fellow, the Jacob Eriksson Prize and the Glenn Anderson Lecture. In 1998, Nobel Peace Laureate Norman Borlaug challenged us to act on global food security, and Peter Scott organized the Task Force on Global Food Security and the Food Security journal. Ahead we have 2020 as the International Year of Plant Health, and a new Task Force on Challenges for Plant Pathology 2050. It has been a fruitful and joyful journey. We have just begun.

Introduction of biocontrol bacteria in potato rhizosphere to prevent latent contamination by pectinolytic bacteria and blackleg symptoms development

E. Munier (1), P. Dewaegeneire (1), J. Cigna (1), V. Helias (2), D. Faure (3), A. BEURY (1), (1) FN3PT/RD3PT, ACHICOURT, FRANCE; (2) FN3PT/RD3PT, Le RHEU, FRANCE; (3) CNRS, GIF-SUR-YVETTE, FRANCE

To control and maintain at low level soil-borne pectinolytic bacteria naturally present in the soil microbiota and to minimize tuber infections, two combined biocontrol strategies for potato plants were developed. These strategies rely on the direct application in-furrow of two bacterial biocontrol agents that were Pseudomonas putida strain A14H7 exhibiting antibiosis activity (Raoul des Essarts et al, 2016) and Rhodococcus erythropolis strain R138 (Cirou et al, 2007) exhibiting a quorum-quenching activity. R. erythropolis R138 strain degrades quorum-sensing signal molecules of the N-acyl homoserine lactone class involved in virulence regulation in pectinolytic bacteria. The effect of this combined treatment on the acquisition of soil-borne pectinolytic bacteria by tubers and expression of blackleg symptoms on plants was evaluated. The two biocontrol agents were monitored over time in the soil rhizosphere during the growing period to ensure that they maintained at detectable levels. The field trials were conducted over three successive field generations. The results showed either an absence of tubers acquisition of pectinolytic bacteria or a decrease of blackleg symptoms in potato plants grown from treated soil. Our data suggest that this biocontrol strategy could provide significant protection of potato plants against pectinolytic bacteria under field conditions.

Suppression of bacterial soft-rot diseases utilizing plant phenolic compounds

I. YEDIDIA (1), J. R. Joshi (2), N. Khazanov (3), H. Senderowitz (3), (1) Agricultural Research Organization, The Volcani Center, RishonLeTsiyon, ISRAEL; (2) The Hebrew University of Jerusalem, Rehovot, ISRAEL; (3) Bar Ilan University, Chemistry Department, Ramat Gan, ISRAEL

Pectobacteria rely on $\mathrm{N}$-acylhomoserine lactone (AHL) signaling to coordinate cell to cell communication and synchronize virulence in a collective behavior termed quorum sensing (QS). Plants in response produce an array of small molecules, several of which have been suggested as QS inhibitors, yet with no resolved mode of action at the atomic scale. Here, QS mutant analysis identified AHL synthase (ExpI), as a preferred target for QS inhibitors. Salicylic acid (SA), the plant defense signal, and the essential oil carvacrol (CAR), were demonstrated to inhibit the accumulation of AHL molecules at concentrations that did not affect bacterial growth or membrane integrity. The phenolics also impaired biofilm formation, exoenzyme activities and reduced bacterial virulence during infection of potato and calla lily. Docking simulations suggest that the phytochemicals interfere with QS by binding of 
SA/CAR to ExpI. The hypothesis was confirmed by Isothermal Titration Calorimetry and Microscale thermophoresis that demonstrated direct binding between the phenolics and purified ExpI. Furthermore, computational Ala scan served to design an ExpI mutant with a predicted weaker affinity to both ligands. Indeed, the computational predictions were confirmed with lower affinity values of SA and CAR to the mutant protein. The direct binding between phytochemicals and bacterial AHL synthase, reinforce interkingdom signaling between plant molecules and bacterial communication systems.

Functional characterization of a large group of CLE effectors encoded by Globodera cyst nematodes

S. CHEN (1), P. Lang (2), M. G. Mitchum (3), X. Wang (2,4), (1) School of Integrative Plant Science, Cornell University, Ithaca, NY, USA; (2) Cornell University, School of Integrative Plant Science, Ithaca, NY, USA; (3) University of Missouri, Columbia, MO, USA; (4) USDA-ARS, Robert W. Holley Center for Agriculture and Health, Ithaca, NY, USA

Like other biotrophic pathogens, plant-parasitic nematodes secrete various effector proteins into host plant cells to facilitate successful infection. CLAVATA3/ESR (CLE)-like effector genes are expressed in various cyst nematode species and their encoded proteins function as mimics of plant CLE peptides to modulate plant developmental pathways that promote the formation of feeding cells needed for the feeding nematode. We previously cloned a large group of CLE genes from four closely related Globodera species, including G. rostochiensis, G. pallida, G. ellingtonae and G. tabacum. These Globodera CLE genes were found to encode predominantly multiple-domain CLE proteins that may result in 37 unique CLE peptides when proteolytically processed in planta. Globodera CLE peptides can be grouped in two classes based on root phenotypes observed in the in vitro peptide application assay: one that suppresses root growth (Group I) and the other that does not arrest root growth (group II). Microscopic observations indicated that several Group II peptides may have an effect in root vasculature. In addition to the StCLV2 receptor, StRPK2, an orthologue of the Arabidopsis RPK2 receptor, was also found to be involved in perceiving PCN-secreted CLE peptides to facilitate nematode parasitism. Further studies are underway to identify other candidate host receptors that are targeted by this large group of nematode-secreted CLE peptides.

Elucidating complex interactions between viruses and vectors: Virus impacts on biology and behavior K. E. MAUCK (1), N. A. Bosque-Perez (2), (1) University of California, Riverside, Riverside, CA, USA; (2) U. of Idaho, Moscow, ID, USA

Dependence on vectors for transmission shapes the evolution of plant virus adaptations for facilitating acquisition, retention, and inoculation. Until recently, it was hypothesized that these adaptations are limited to virus proteins that enable virion binding to vector mouthparts or invasion of internal tissues. However, we now have evidence that viruses can manipulate host plant phenotypes in ways that enhance transmission by vectors. Viruses influence vector behavior through alteration of plant cues that mediate vector orientation, feeding, and dispersal behaviors. These cues include tactile and visual aspects, odors, defenses, secondary metabolites, sugars, and free amino acids. Effects are not uniform, but exhibit convergence depending on the specific frequency and duration of probing and feeding required to transmit distinct types of plant viruses. The induction of similar phenotypes by phylogenetically divergent viruses transmitted via the same sequences of vector behavior supports the hypothesis that virus effects are not just byproducts of infection. Instead, effects are purportedly induced by multifunctional proteins that, in addition to primary roles in host plant exploitation, have evolved secondary functions as effectors of host phenotypes. The question of whether viruses can evolve manipulative functions in natural or agricultural settings is central to our understanding of the ecological and epidemiological importance of host and vector manipulation. To address this question, we synthesized available literature to identify patterns in virus effects among pathogens with shared transmission mechanisms. Results are discussed in the context of molecular and environmental constraints on virus evolution, limitations of existing studies, and prospects for future research.

Prospects and challenges of High Throughput Sequencing for viral pathogen diagnosis and expedited release of quarantined propagative plant material

M. AL RWAHNIH PHD (1), D. A. Golino (2), (1) Foundation Plant Services Facility, University of California, Davis, CA, USA; (2) University of California, Davis, CA, USA

High Throughput Sequencing (HTS) provides a rapid, robust approach for viral plant pathogen identification. Recent studies found HTS superior to conventional methods for detecting viruses of economic significance in grapevine and fruit trees. As such, Foundation Plant Services has an import permit that allows provisional release of propagative plant material that has been HTS screened for pathogens. When HTS is used in conjunction with current conventional methods, growers of certified and registered material can initiate propagative increase and virus elimination programs with new accessions years earlier. While HTS remains a powerful new technology with significant benefits for plant certification and quarantine programs, there are challenges to consider. Since detecting a given pathogen sequence does not mean that pathogen is responsible for the disease, establishing biological significance for viruses identified via HTS analysis is necessary. Biological effects are assessed by graft transmission, completion of Koch's postulates, spread and distribution studies, and determining the agronomic significance of the symptoms. In addition, efficient sample preparation methods for large scale application and bioinformatics algorithms to efficiently separate pathogen and host sequences must be developed, validated, and standardized across laboratories. No regulatory decision can be made on the importance of a novel virus without information on its biological effects.

Future directions in agricultural technology transfer for plant disease management A. R. RECORDS, USAID, Washington, DC, USA

Feed the Future (FTF), the U.S. Government's global hunger and food security initiative, works with partner countries to develop their agriculture sectors and break the vicious cycle of poverty and hunger. The U.S. Agency for International Development (USAID) leads FTF and its research agenda, which includes addressing emerging plant diseases - like coffee rust in Central America and wheat rust in Africa and Asia—which can have devastating impacts in developing countries, where the livelihoods and food security of smallholder farmers and their families depend on healthy plants. An important component of FTF is support for research to develop agricultural innovations. These innovations, once proven effective and ready for implementation at the field level, are ripe for scale up, which depends on the establishment of strong and complementary partnerships and often on organizational and institutional change. In recent years, USAID has increasingly focused on the translation of research investments into population level impacts through the systematic identification of scale up-ready technologies, surveys on research uptake, and the development of a scaling assessment tool. Past successes and future directions in scaling and technology transfer will be discussed in the context of plant disease.

Digital aerial assessment of turfgrass pests for precision management and monitoring epidemics D. S. MCCALL (1), J. Booth (2), D. Sullivan (3), (1) Virginia Tech, Blacksburg, VA, USA; (2) Virginia Tech, Moseley, VA, USA; (3) TurfScout, Greensboro, NC, USA

Digital image analysis (DIA) drives artificial intelligence that has revolutionized modern society. Applications are increasingly common in the medical, automotive, and agricultural industries. Agricultural remote sensing relies on raw and transformed digital spectral properties. For instance, vegetation 
indices typically utilize a ratio of visible and near infrared light to discern relative plant phenology. Popularity of aerial imagery collected from drone platforms has increased recently in the turfgrass industry, though practical use of the data is still in its infancy. Large datasets from drone-based DIA comes with unique challenges associated with lighting and spatial alignment that are more controlled with ground measurements. Our lab has developed methods to spatially map pest outbreaks using aerial DIA and computer recognition software. Best data and mosaic alignment is achieved on overcast days that improve contrast and reduce shadows. Glare from sunny days create exposure problems and can be minimized by imaging at solar noon. Mosaicked images should include $>50 \%$ overlap to provide useful redundancy. Ground reference points with high spatial accuracy will improve georectification of mosaicked images. Digital recognition of ground-validated disease symptoms is possible through computer learning approaches. Geospatial disease maps can reduce pesticide inputs through targeted applications and offer a powerful model for improved epidemiological investigation.

Spectral characterization of bacterial leaf blight of rice through spectroscopy and remotely sensed multi-spectral imagery R. T. ALBERTO, Central Luzon State University, Science City of Munoz, Nueva Ecija, PHILIPPINES

Bacterial Leaf Blight is one of the most destructive diseases of rice. Detection at early growth stage may prevent substantial damage and yield loss. Since all objects on earth's surface has its own spectral reflectance, spectral reflectance curves of both bacterial leaf blight infected and uninfected rice leaves were compared and assessed the possibility of using remote sensing imagery to determine which spectral bands are most responsive to the presence of BLB. Spectroscopy and multi-spectral imaging were applied to rapidly and non-destructively characterized BLB-infected rice fields. Spectral signatures of rice leaves at different severity levels were collected, namely: normal (healthy), slight, moderate to severe. The data were processed using spectral calculator to obtain the ratio between reflectance and wavelength within the electromagnetic spectrum. Multi-spectral Landsat 8 Operational Land Imager imagery was also downloaded in Geotiff format and pre-processed in ENVI software using radiometric calibration. Leaf spectroscopy showed greater variation between healthy and BLB infected rice plant with wavelengths between 650 to 680 nanometer (red region) and 749 to 769 (NearInfrared region). In Landsat 8 image, wavelengths from 630 - $670 \mathrm{~nm}$ (red region) were statistically separable indicating BLB infection, suggesting that both leaf spectroscopy and multi-spectral images can discriminate healthy and BLB-infected rice, thus, the possibility of using multi-spectral images to estimate BLB infection in a larger scale, estimating potential losses and allowing precision disease management. Moreover, using high resolution images could further characterize diseases of different severity levels to avoid spectral mixing caused by medium resolution multispectral image.

Spatial and ecological heterogeneity affects disease development in forests: Disease disturbance interactions R. COBB (1), M. Metz (2), (1) Cal Poly State University, San Luis Obispo, CA, USA; (2) Lewis and Clark College, Portland, OR, USA

Plant pathology has a long history of uncovering environmental drivers of disease emergence many of which have been integrated into research and management of human and zoonotic diseases. As plant pathology has advanced understanding and management of plant disease with the disease triangle familiar to pathologists, the field of landscape ecology has integrated analogous factors to understand the factors that shape, define, and alter large-scale systems. Advances in both fields has laid bare persistent problems in management of plant disease in open systems and revealed hidden drivers of landscape change: the interaction of broad spatial-scale events that drive or modify the components of the disease triangle, sometimes in surprising and unanticipated ways. We combine newly emerging theory and field study of disturbance interactions from landscape ecology with the traditional perspective and insights of plant pathology. Some theory-based progress linking these fields has been achieved over the last 10-20 years, but in the meantime, wildland tree diseases have been altered by other broad-scale disturbances such as climate change, fire, landuse, and insects. We examine the effects of sudden oak death on fire through changes in fuels, their flammability, decomposition rates, and extent of accumulation. We also examine interacting disturbances as disease drivers for several other forest diseases where empirical data is lacking, but where theory or epidemiological models suggest disturbance interactions almost certainly occur.

Exception to the norm: Inactive TAL effectors trigger an atypical resistance mechanism in rice A. I. HUERTA (1), L. R. Triplett (2), T. Borland (1), J. E. Leach (1), (1) Colorado State University, Fort Collins, CO, USA; (2) Department of Plant Pathology and Ecology, The Connecticut Agricultural Experiment Station, New Haven, CT, USA

Plant pathogenic Xanthomonas spp. produce numerous transcription activator-like effectors (TALEs) induce expression of host susceptibility genes. TALE-DNA binding is conferred by the central repeat region (CRR) which pairs one repeat to one DNA base in the promoter of host genes. Plants have evolved TALE-associated resistance mechanisms to circumvent pathogen attack, which include dominant TALE-dependent resistance, loss of susceptibility, and dominant non-transcriptional resistance. Comprised in the latter are resistance loci Bs4 and the recently described Xo1 from tomato and rice, respectively. The resistance mechanism of both Bs4 and Xo1 is hypothesized to be dependent on cytoplasmic recognition of the highly conserved CRR but its mechanistic details remain unknown. To better understand how TALE trigger or escape Xo1 detection we aligned the CRR protein sequences of eight Xo1 triggering TALE with two non-triggering effectors. We found consistent variation in the amino acid (AA) residues at position 4, 8, 33 and 35. In addition, these same AA residues were predicted to be solvent exposed. We tested the role of these key AAs in triggering Xo1 by swapping AA residues at these key positions in resistance- and susceptibility-inducing TALEs. A mutated resistance-inducing PthXo1 TALE lost its resistance-triggering ability, indicating that Xo1 recognition is sensitive to solvent exposed AA residues. The strong similarity between Xo1 and Bs4 TAL effectors triggered resistance phenotype suggest that monocots and dicots may share an ancient or convergently evolved mechanism to recognize the conserved structure of TAL effectors.

\section{Beneficial fungal endophytes in cotton}

G. SWORD (1), M. V. Kolomiets (1), E. J. Borrego (1), C. Suh (2), C. Gale (1), (1) Texas A\&M University, College Station, TX, USA; (2) USDA-ARS, College Station, TX, USA

Beneficial fungal endophytes can confer protection to plants from a variety of stressors and improve yields in major agricultural crops. Fungal endophytes originally isolated from cultivated cotton (Gossypium hirsutum) have been shown to have a number of important ecological, physiological and agronomic effects on the plant. Using simple seed treatment protocols, individual cotton plants can be inoculated with endophytic fungi with resulting phenotypic effects detectable across the entire growing season. The targeted manipulation of fungal endophytes in cotton can mediate resistance to multiple stressors including insects, nematodes and drought, with significant positive impacts on plant performance and yields in the field. Having demonstrated that the application of endophytic fungi to cotton can impact plant responses to multiple stressors, ongoing work is focused on the mechanisms by which the fungi exert these effects in cotton, and comparative analyses of their effects in other plants.

Diversity of biocontrol-related traits revealed by whole-genome analysis of worldwide-isolated phenazine-producing Pseudomonas spp A. BIESSY, A. Novinscak, G. Léger, M. Filion, Université de Moncton, Moncton, NB, CANADA 
Plant-beneficial Pseudomonas spp. aggressively colonize the rhizosphere and display plant-growth promotion and/or disease-suppression activities. Some plant-beneficial Pseudomonas spp. strains within the $P$. fluorescens species complex produce several phenazine derivatives, such as phenazine-1carboxylic acid (PCA), phenazine-1-carboxamide acid ( $\mathrm{PCN}$ ) and 2-hydroxyphenazine (2-OH-PHZ), which are inhibitory to numerous soilborne plant fungal and bacterial pathogens. In this project, we assembled a collection encompassing 63 representative strains of the worldwide diversity of plantbeneficial phenazine-producing Pseudomonas spp. Genome sequencing and comparative genomics revealed an important diversity, illustrated by a large pangenome accounting for 25413 protein-coding genes. It also displayed insight into the evolutionary history of the phenazine biosynthetic operon. Given its diverse genomic context, it is likely that this operon was relocated several times during Pseudomonas evolution or independently acquired by horizontal gene transfer. We also identified numerous biocontrol-related traits, including antibiotics, siderophores and cyclic lipopeptides biosynthesis, some of which were previously unknown in these microorganisms. Strains were further characterized for their role in the biocontrol of three major potato pathogens, Phytophthora infestans, Verticillium dahliae and Streptomyces scabies.

The making of tree pathogens: Big data approach

R. HAMELIN, University of British Columbia, Vancouver, BC, CANADA

Tree pathogens are responsible for some of the most devastating outbreaks in forests. In spite of their importance, we know relatively little about their origin and sources, and their basic common characteristics. A fundamental question in forest pathology is what makes a tree pathogen? Pathogenic species are often phylogenetically related to non-pathogenic species, which provides an opportunity to identify the determinants of pathogenicity. By harnessing the power of genomics we can compare fungi with a broad range of life-style, host specificity and pathogenicity. The Pathobiome and BioSAFE projects involve sequencing and comparing the genomes of multiple pathogens of trees to identify common and unique genomic determinants that can be developed into tools for monitoring and risk prediction. The genome of the wood-infecting poplar canker pathogen Sphaerulina musiva was compared to the genome of a close relative that lacks that ability (i.e. S. populicola). Sphaerulina musiva has acquired and expresses a gene arsenal, such as hemicellulose-degrading enzymes, peptidases, and metabolite transporters that are required for colonization of woody tissues. A secondary gene cluster that is activated upon interaction with the host and several carbohydrate degradation genes have the signature of horizontal transfer from both ascomycete fungi associated with wood decay and prokaryotes. These results suggest that a dynamic genome landscape can drive pathogenic potential.

Unravelling the soil immune response

V. CARRION (1), J. Raaijmakers (2), (1) Netherlands Institute of Ecology, Wageningen, NETHERLANDS; (2) Netherlands Institute of Ecology, 6708 PB Wageningen, NETHERLANDS

Soil and plant microbiomes contribute to a range of plant support functions, including nutrient acquisition and disease protection. To date, the molecular mechanisms involved in natural disease suppressive soils have primarily been studied for single microbial strains isolated from the rhizosphere and endosphere. The role of microbial consortia in this soil immune response is much less studied. Here, we conducted a microbiome-wide analysis of the taxonomic diversity and functional potential of the endosphere of plants grown in a soil that is naturally suppressive to damping-off disease caused by the fungal root pathogen Rhizoctonia solani. Metagenomic analyses showed a significant enrichment of Chitinophagaceae, Flavobacteriaceae, Burkholderiaceae, Pseudomonadaceae and Xanthomonadaceae families in the endosphere of plants challenged with the fungal pathogen. Binning of the metagenomic sequences allowed de novo assembly of mulitple high quality genomes of different endophytic bacterial genera. Subsequent trait-based analyses revealed numerous biosynthetic gene clusters (BGCs) overrepresented in the endophytic bacterial families of plants under pathogen attack, including BGCs involved in signal transduction, extracellular enzyme and secondary metabolite production. Network analyses further indicated that the traits enriched in the endophytic microbiome, in particular nonribosomal peptides and polyketides, involve several novel BGCs encoding metabolites with yet unknown structures and activities. Bioassays further showed that consortia of endophytic strains and not single strains conferred disease protection to the host plant. Collectively these results indicate that endophytic microbial consortia with diverse functional traits contribute to the soil immune response.

Earth laughs in flowers: How emergent downy mildew diseases are affecting the ornamental crop industry and what to do about it C. SALGADO-SALAZAR (1), N. LeBlanc (2), J. A. Crouch (3), (1) Mycology and Nematology Genetic Diversity and Biology Laboratory, USDAARS, Beltsville, MD, USA; (2) USDA-ARS, Beltsville, MD, USA; (3) USDA ARS, Beltsville, MD, USA

Downy mildews are some of the most devastating diseases of ornamental plants. While many downy mildews are caused by pathogens previously unknown, others have been around since the $19^{\text {th }}$ century, only to recently re-emerge as destructive pathogens exhibiting unwelcome changes in virulence, host range and epidemiology. Newly emergent downy mildew pathogens affecting rudbeckia and impatiens exemplify some of the new challenges faced by the horticultural industry. Plasmopara halstedii emerged as a destructive pathogen of cultivated ornamental rudbeckia in the late 1990's, although this pathogen was known as an inhabitant of native rudbeckia and presumed to be the same pathogen causing sunflower downy mildew. Using population genetics, we show that isolates infecting rudbeckia are genetically distinct from isolates infecting sunflower, constituting an entirely different species. In the case of the pathogen responsible for impatiens downy mildew epidemics beginning in 2004, we identified seven different genetic populations of the causal pathogen P. obducens, with clear genetic differences between pre-and post-epidemic populations. Here we discuss the knowledge gaps presented by these emergent diseases and the need for further integration of information that would aid disease mitigation efforts. These include the revised methods for species identification and disease diagnostics, identification of common patterns and drivers of emergent and reemergent diseases, and the development of improved disease management strategies.

Generating disease resistant citrus varieties using CRISPR/Cas9

N. WANG, University of Florida, Lake Alfred, FL, USA

Citrus is one of the most important fruit crops worldwide. However, citrus industry faces multiple disease challenges, including citrus Huanglongbing (HLB) caused by Candidatus Liberibacter asiaticus (Las) and citrus canker caused by Xanthomonas citri subspecies citri (Xcc). Generating disease resistant citrus varieties is an efficient and sustainable term solution to either HLB or canker disease. However, the traditional approach of breeding for resistant plants for citrus is difficult and has not provided a timely solution to control HLB or canker due to the long juvenility, large tree size, and prevalence of polyembryonic seeds in commercial varieties. Here I will present our progress in generating disease resistant citrus varieties via CRISPR technology. The application of different CRISPR tools on citrus genome editing will be presented. I will also present our results on identification of suitable targets for resistance development against either HLB or canker. 
Uncovering molecular mechanisms of pathogenicity in the banana pathogen Fusarium oxysporum f.sp. cubense

E. CZISLOWSKI (1), I. Zeil-Rolfe (2), S. Fraser-Smith (2), J. Dalton-Morgan (3), E. Aitken (1), (1) School of Agriculture and Food Sciences, The University of Queensland, Brisbane, AUSTRALIA; (2) School of Agriculture and Food Sciences, St. Lucia, AUSTRALIA; (3) University of Queensland, St Lucia, AUSTRALIA

Banana is an incredibly important crop due to its value as a subsistence crop for millions of people throughout the world as well as its value as an export commodity for many countries. The ongoing cultivation of banana in all growing regions is under threat from the hypervirulent Tropical Race 4 (TR4) variant of the fungal pathogen, Fusarium oxysporum f.sp. cubense (Foc). Currently, it is hypothesised that the host-specific pathogenicity of fungal pathogens is influenced by small, secreted proteins termed effectors. This study sought to understand the repertoire of effector genes present in lineages of $F O C$ and how these genes have influenced the evolution of this pathogen. Using a systematics approach, we investigated the distribution and diversity of a family of fungal effectors named the secreted in xylem (SIX) genes in the different genetic lineages of Foc. We identified homologues of several SIX genes that were specifically associated with the TR4 lineage of Foc. Sampling of Fusarium spp. associated with asymptomatic bananas also revealed that endophytic isolates of F. oxysporum, and interestingly other Fusarium spp., carried several SIX genes. Hypothesis testing indicated that the SIX genes in banana-infecting isolates of $F$. oxysporum had been acquired through horizontal gene transfer events. To investigate this further, we have undertaken a comparative genomics approach using TR4 and two less pathogenic lineages to identify regions of the Foc genome implicated in pathogenicity. This study has important implications for understanding the molecular mechanisms of pathogenicity in Foc and more specifically the molecular factors that confer the hypervirulence of TR4.

Genome-based classification and identification of bacteria

L. Tian (1), Y. Vasebi (2), V. Eastman (2), K. Hirani (2), G. Hughes (2), L. Heath (3), B. A. VINATZER (2), (1) Department of Plant Pathology, Physiology, and Weed Science, Virginia Tech, Blacksburg, VA, USA; (2) Virginia Tech, Blacksburg, VA, USA; (3) Department of Computer Science, Virginia Tech, Blacksburg, VA, USA

The number of sequenced bacterial genomes in public databases has surpassed the number of bacterial strains in culture collections. Although complete genome sequences offer the potential to precisely and correctly identify the associated bacteria, many of these genomes are assigned to the wrong species because of errors made by who submitted the genome sequences or subpar database curation. Moreover, even if all genomes in public databases were correctly identified at the species level, genomes of many beneficial or pathogenic plant-associated bacteria would still be of dubious identity. In fact, individual plant-associated bacterial species encompass vast genotypic and phenotypic diversity based on which isolates can be assigned to intraspecific classes such as pathovars, races, biovars, phylotypes, sequevars, clades, and many more. To address this immense challenge, we have designed LINbase, a database that automatically assigns genomes to genome similarity groups based on their Life Identification Numbers (LINs). These genome similarity groups can either be automatically associated with named species and intraspecific classes based on metadata of individual genomes or users can make these associations manually. The result is a tool that allows precise classification and identification of plant-associated bacteria at the species and intraspecific level with applications in plant biosecurity, plant disease diagnostics, and phytobiome research.

Simple Models of Durable Resistance to Plant Diseases

R. L. BOWDEN (1), T. C. Todd (2), K. A. Garrett (3), B. Valent (2), (1) USDA ARS, Manhattan, KS, USA; (2) Kansas State University, Manhattan, KS, USA; (3) Plant Pathology Department, University of Florida, Gainesville, FL, USA

Durable resistance to diseases is the ultimate aim of many crop improvement programs, however, a stronger theoretical basis is needed to inform decision-making about strategies for achieving durability. We developed two simple models that describe how durability can be a result of either: 1) a low probability that virulent pathogen genotypes arise from mutation or recombination, or 2) low relative fitness of those virulent genotypes. In the first model, the probability of occurrence of new virulent pathogen genotypes is a negative exponential function of population size, mutation or recombination rate, and establishment efficiency. In the second model, durability is predicted when the selective advantage associated with defeat of the resistance gene is less than the fitness cost of virulence. Likely success or failure of any specific strategy for durable resistance can be evaluated if the model parameter values can be estimated for a given situation. We also propose guidelines for assessing the intrinsic durability of resistance genes and designing durable combinations of qualitative genes or combinations of both qualitative and quantitative genes.

Classification and characterization of plant viruses identified by metagenomics approaches

J. K. BROWN, School of Plant Sciences, University of Arizona, Tucson, AZ, USA

The application of genomic pathology tools for the 'discovery' of new and emerging plant viruses is expected to have far-reaching effects in the field of plant virology. Advancements in metagenomics have created unprecedented potential for discovering previously unknown or unrecognizable viruses infecting plants on land and in the seas. However, is not clear how annotation of coding regions or the assignment of gene function for the anticipated plethora of genomic variants will be accomplished, in part, because most will not be established in culture, cloned, or archived as in the past. For pathogenic viruses, this could delay proof of causality and timely fundamental studies. Also, the sheer number of viral genome sequences destined for public databases is expected to grow exponentially, requiring additional storage, and increased capacity in taxonomic frameworks for already-recognized and entirely new taxa. Further, the pace at which the criteria guiding taxonomic classification and rules of nomenclature will substantially accelerate. At the same time, metagenomics technologies offer enormous potential for uncovering the dynamics of entire viral populations, in near, real-time. Such information, shared through global networks, could reduce viral spread or prevent outbreaks altogether. Use of extensive sequence databases will accelerate the association of new viruses with outbreaks and of arthropod or other vectors with transmission, leading to expedited disease management with increased precision. The rapid expansion in 'viral genome real estate' is creating exciting, new possibilities for mining uncharted territories to expand fundamental knowledge in plant virology, and facilitate timely discovery, tracking, and management of viral diseases.

An analytical framework for consistent evaluation of pest and disease management technologies J. MUMFORD (1), A. Leach (1), P. Baranowski (1), J. Holt (1), B. Agstner (2), G. Jones (2), J. Alden (1), M. Quinlan (1), (1) Imperial College London, Ascot, UNITED KINGDOM; (2) Fera Science Ltd, York, UNITED KINGDOM

EMPHASIS is developing a range of technologies for detection and control of agricultural pests and diseases. The Analytical Framework is a spreadsheet-based tool to facilitate consistent evaluation of these technologies under four broad categories of performance indicators: control efficacy; coherence and relevance; economic efficiency; environment and sustainability. There are 30 more specific indicators differentiated within these categories. The Analytical Framework uses a unique method of elicitation to collect and assess information about early-stage and rapidly-developing 
technologies. At the project level this enables evaluation of the strengths and weaknesses of specific technologies. The tool can be used to make comparisons with existing practice and provides a dynamic framework (in an updatable spreadsheet) to link technology descriptions, trials results, user learning and potential commercial plans for the measures. At the individual level the Analytical Framework enables developers to assess their technology against performance indicators, identifying individual strengths and weaknesses, so helping the technology progress towards commercialisation and uptake. A Technology Readiness Level tool allows assessment of this progress. Together these tools provide the first step in business planning that combines technological performance, cost and market projections.

The rough end of the pineapple: The sometimes prickly relationship between science and policy A. YOUNG, The University of Queensland, Gatton, AUSTRALIA

A key component of food security is the restriction of movement of plant pathogens. The goal of maintaining area freedom from plant diseases can sometimes be compromised by trade decisions that are made with limited reference to the scientific community. When this happens, primary industries are put at risk of incursion events. This presentation focuses on lessons gleaned from the interaction between Australian biosecurity agencies and scientific agencies. Specifically, it investigates the role of an incomplete bacteriological nomenclature when assessing the import risks of pineapples into the Australian market. Using a dated and incomplete taxonomic framework, Australian biosecurity agencies claimed that the bacterium that causes heart rot of pineapples in Malaysia (now known to be specific strains of Dickeya zeae), was already present in Australia, where it caused bacterial mottle in sugarcane, nominally caused by Pectobacterium chrysanthemi. Although this was a clear case of the scientific nomenclature lagging behind scientific knowledge, this demonstrates the need for greater integration between policy makers and the scientific community, and the need to ensure that the best possible scientific evidence is incorporated into the decision making framework. This presentation discusses improvements that can be made in international trade in agricultural products.

\section{Innovative detection methods to support plant health diagnostics}

P. J. M. BONANTS (1), I. Houwers (1), A. Dullemans (1), Y. Griekspoor (1), O. Mendes (2), M. Gent van (1), R. A. Van Der Vlugt (1), J. Bergervoet (1), C. D. Schoen (1), J. van der Wolf (1), T. van der Lee (1), (1) Wageningen Plant Research, Wageningen, NETHERLANDS; (2) Wageningen University and Research, Wageningen, NETHERLANDS

International trade and travel have increased tremendously in recent years, with plants and plant products being moved around the whole world. As a consequence, the rate of introduction and establishment of new economically or environmentally damaging plant organisms and invasive species has increased steadily. The ability of National Plant Protection Organizations (NPPOs) and their affiliated laboratories to quickly and reliably detect and identify organisms is critical for the effective performance of phytosanitary measures. Plant pest diagnostics is also essential to support the phytosanitary certification of consignments of plants and plant products exported from countries. Use of efficient methods to detect all these organisms is essential in this whole process. Also method validation and the access to reference material for use as positive controls ensure the reliability of diagnostics in international setting. In this paper we address several innovative diagnostic methods: e.g. TaqMan PCR, Luminex, LAMP and Next Generation Sequence will be discussed in different applications.

Climate as a predictor of microbiome diversity in Neotropical forests of Mexico in the Anthropocene L. VILlaRREAL RUIZ (1), C. Neri Luna (2), (1) Colegio de Postgraduados, PREGEP-Genetics Department, LARGEMBIO, Texcoco, MEXICO; (2) Universidad de Guadalajara, CUCBA, Ecology Department, Plant ecophysiology Lab, Nextipac, MEXICO

The tree rhizosphere contains a diverse and overlooked microbiome that could influence the effect of climate change on plant health. Here, we studied four geographically and climatically different Neotropical forests, including: two tropical coniferous montane forests (Pseudotzuga menziesii); one dry tropical forest (Neea psychotrioides); and one chaparral (Quercus potosina-Archtostaphylos pungens) forest, located in east-central and southwest, southeast, and north-central Mexico, respectively. At each location, soil samples were collected from 20 randomly selected trees using soil cores. Samples $(\mathrm{n}=160)$ were taken at a distance of 1-1.5 m from the trunk on opposite sides of each tree, and analysed with 454 pyrosequencing, bioinformatics, quantitative and biogeographic methods. Metabarcoding analysis revealed a total of 10,463 species-level OTUs of eukaryotic microbial diversity in the rhizosphere. Fungi represented $95.7 \%$ of the species-level OTUs followed by Metazoa (1.5\%), Viridiplantae (1.3\%), Protista: AlveolateRhizaria-Amoebida (1.2\%), and $0.25 \%$ with no identified Kingdom. The main fungal functional groups were mycorrhizae $73 \%$, mycoparasites $13.7 \%$, plant pathogens $8.5 \%$, white-rot fungi $4.2 \%$ and animal parasites $0.5 \%$. Differential changes in organismal and fungal functional group diversity were observed in the four sites, and climate was the best diversity predictor, likely due to climatic effects on plants, fungi and possibly other soil microbial communities.

Anaerobic soil disinfestation for management of soilborne diseases in muck soil vegetable production systems A. L. TESTEN (1), S. A. Miller (2), (1) The Ohio State University, Wooster, OH, USA; (2) Department of Plant Pathology, The Ohio State University, Wooster, OH, USA

Anaerobic soil disinfestation (ASD) was evaluated as a tool for managing clubroot (Plasmodiophora brassicae) in mustard greens and root knot nematodes (Meloidogyne hapla) in lettuce produced on muck soils in the Midwestern United States. Wheat bran (20.2 t/ha), molasses (10.1 t/ha), and wheat bran $(20.2 \mathrm{t} / \mathrm{ha})$ plus molasses $(10.1 \mathrm{t} / \mathrm{ha})$ were assessed as ASD carbon sources and compared to non-amended, uncovered and covered control plots in two consecutive years of field trials. Post-ASD bioassays were also conducted in controlled environments using soils treated by ASD in the field. A significant reduction in clubroot disease index was observed in only one year of field trials, but significant reductions in disease index were observed in three of four post-ASD bioassays. Significant increases in mustard biomass were observed plants grown in wheat bran-amended soils in one year of field trials and in three of four bioassays. All ASD treatments significantly reduced nematode galling on lettuce, with up to $86 \%$ and $98 \%$ reduction in field trials and post-ASD bioassays, respectively. Significant increases in lettuce biomass for wheat bran-based treatments were not observed consistently in field trials, but were observed in all bioassays. The results of these studies indicate that ASD is a promising tool for managing soilborne diseases in muck soil vegetable production systems, yet ASD must be further optimized before it is widely adopted by growers.

Accessory chromosomes and host-specific pathogenicity in Fusarium oxysporum M. REP, University of Amsterdam, Amsterdam, NETHERLANDS

The Fusarium oxysporum species complex (FOSC) is notorious for harbouring plant-pathogenic clonal lines that cause wilt or root/bulb rot in crops and ornamentals. However, pathogenicity is rare in the FOSC. Colonization of roots by Fusarium oxysporum without negative consequences for plant health appears to be very common in most plant species investigated. It appears that monoculture has selected rare pathogenic clonal lines, providing a 
'substrate' for continued spread of those lines, with Panama disease of banana as a dramatic example. We have discovered that host-specific pathogenicity resides in accessory 'pathogenicity chromosomes' and is at least partly encoded by genes for small secreted proteins ('effectors') on these chromosomes. On the basis on the repertoire of putative effector genes in a genome of a strain of Fusarium oxysporum, we can now predict host-specific pathogenicity with high confidence for an increasing number of plant hosts, starting with tomato and cucurbits. Pathogenicity chromosomes can be transferred horizontally between strains, explaining why many host-specific forms (formae speciales) consist of multiple clonal lines. We have indications that this horizontal chromosome transfer occurs in a heterokaryon emerging from hyphal fusion between 'incompatible' strains in which rare nuclear fusion is followed by selective loss of 'core' chromosomes from one parental strain.

\title{
Fungicide efficacy and molecular characterization of North Carolina Colletotrichum populations causing Glomerella leaf spot and fruit rot on apple
}

K. JOHNSON, R. Kreis, C. Justus, S. M. Villani, North Carolina State University, Mills River, NC, USA

Glomerella leaf spot (GLS) and fruit rot (GFR) are caused by fungi in the Colletotrichum gloeosporioides species complex. Knowledge gaps in causal species, pathogen biology, and fungicide efficacy have made management of GLS and GFR extremely challenging for North Carolina (NC) apple growers. To more effectively manage GLS and GFR during the growing season and post-harvest, field experiments investigating fungicide efficacy were conducted in a 'Tenroy Gala' research orchard in 2017. Fungicides representing different modes of action were applied in non-rotational programs from petal fall through 9th cover. Merivon (pyraclostrobin + fluxapyroxad) applications resulted in lower incidence and severity of GLS and GFR, but were not significantly different than multi-site fungicide applications $(p \leq 0.0001)$. Additionally, 433 isolates of Colletotrichum spp. were collected from diseased fruit and leaves in NC commercial apple orchards for species identification and sensitivity to the quinone outside inhibitor (QoI) fungicides pyraclostrobin and trifloxystrobin. Initial multigene sequence analysis of 18 fruit isolates and 14 leaf isolates revealed $C$. fructicola as the most prevalent species causing GLS and GFR in NC. Currently, in vitro mycelial and conidial germination assays are being conducted to evaluate QoI fungicide sensitivity of species within the C. gloeosporioides complex and between Colletotrichum spp. populations in NC.

Quantitative methods for the improved surveillance of emerging plant pathogens; an epidemiological approach S. R. PARNELL (1), T. R. Gottwald (2), A. Mastin (1), N. J. Cunniffe (3), C. A. Gilligan (3), F. Van den Bosch (4), (1) University of Salford, Salford, UNITED KINGDOM; (2) USDA-ARS, Ft Pierce, FL, USA; (3) University of Cambridge, Cambridge, UNITED KINGDOM; (4) Rothamsted Research, Harpenden, ENGLAND

The rise in the number of newly-introduced plant pathogens in recent times has placed an increasing emphasis on effective surveillance strategies for the detection and monitoring of disease. We advocate a quantitative-based approach to surveillance and demonstrate how our work, that captures the epidemiology of pathogen populations, can be used to better target the use of surveillance resources. We outline a range of novel quantitative methods that allow us to address crucial questions in the surveillance of emerging diseases. For example: Following the first discovery of a disease in a new area, what prevalence will it have reached in the population? How often and how intensively do we need to survey to discover a new epidemic at a sufficiently early stage? In host-vector systems, should we focus early-detection survey efforts on sampling insect populations or sampling host plants? When disease risk varies across a landscape, where and when should we target our sampling efforts for improved detection? How can we optimize the design of surveys to improve mapping of invading pathogen populations? Finally, we discuss how an epidemiological-based approach can lead to the improved deployment and use of valuable new detection technologies, such as sensitive field-based diagnostics and remote sensing.

Assessment of crop health and losses to plant diseases in world agricultural foci

A. NELSON (1), S. S. Savary (2), L. Willocquet (2), P. Esker (3), S. J. Pethybridge (4), N. McRoberts (5), (1) University of Twente, Enschede, NETHERLANDS; (2) INRA, Castanet Tolosan, FRANCE; (3) Penn State University, UNIVERSITY PARK, PA, USA; (4) Cornell University, Plant Pathology \& Plant-Microbe Biology Section, Geneva, NY, USA; (5) University of California, Davis, CA, USA

In November 2016, the ISPP Crop Loss Subject Matter Committee initiated a three-month online global survey of crop losses. The global survey appears to be the first collective expert assessment of the importance of losses caused by diseases and pests of the world's five most important food crops; wheat, rice, maize, soybean and potato. This voluntary survey on the location, frequency and magnitude of crop losses caused by diseases and pests generated 990 responses from 216 experts in 67 countries. These responses were used to generate expert-based estimates of crop losses using a three step procedure: (i) an assessment of the representativeness and validity of the survey results by comparing the reported losses and geographic distribution of diseases and pests to those in the CABI Crop Protection Compendium datasheets and other references; (ii) computation of average losses per disease or pest per country for each country that featured in the survey results and imputation of losses for countries where the disease or pest was known to occur but was not reported, and; (iii) adjustment of these average losses based on national crop production statistics and the ecology and management of each disease and pest. This talk presents the estimated losses by crop and by disease or pest, both globally and across eight geographic food security foci. We compare these new estimates with existing estimates and discuss the importance and validity of this global crop loss assessment.

\author{
Using Predictions from a Fusarium Head Blight Risk Assessment Tool as Predictors of the risk of Deoxynivalenol Contamination of Wheat \\ Grain
}

W. BUCKER MORAES (1), E. D. De Wolf (2), D. A. Shah (2), J. D. Salgado (1), L. V. Madden (1), P. A. Paul (1), (1) The Ohio State University, Wooster, OH, USA; (2) Kansas State University, Manhattan, KS, USA

Fusarium head blight (FHB), caused by Fusarium graminearum, is associated with wheat grain contamination with mycotoxins such as deoxynivalenol (DON). A pre-anthesis weather-based tool was previously developed to help guide fungicide applications for FHB management based on predicted risk of an FHB epidemic. However, producers and grain buyers also use FHB predictions as indicators of the risk of DON contamination when making grain marketing decisions. But the accuracy of the FHB risk tool for DON prediction has not been formally evaluated. Here we used the predicted risk of an FHB epidemic (FHB-RISK) as an independent variable to predict DON contamination above a grain marketing threshold of $>2 \mathrm{ppm}$. A total of 583 unique cases was used to build a logistic regression model to estimate the probability of DON exceeding 2 ppm when the predicted risk of an FHB epidemic was low, moderate, or high (FHB index $>10 \%$ ). The area under the ROC curve, a measure of model discrimination between cases with and without DON $>2 \mathrm{ppm}$, was 0.66 , and the overall correct classification rate was $70.7 \%$. The sensitivity (proportion of cases with DON $>2 \mathrm{ppm}$ correctly classified) and specificity (proportion of cases with DON $\leq 2 \mathrm{ppm}$ classified correctly) were 39.3 and $92.4 \%$, respectively. These results suggest that one should not rely on FHB risk predictions alone to assess the risk of DON contamination as such predictions performed poorly classifying cases with DON levels $>2$ ppm. 
Advantages of mobile and smart spore traps in disease surveillance

R. Kimber (1), L. Zeller (2), S. Wili (3), J. WEST (4), (1) South Australian Research and Development Institute, Adelaide, SA, 5001, AUSTRALIA; (2) University of Southern Queensland, Toowoomba, AUSTRALIA; (3) Burkard Manufacturing Co. Ltd., Rickmansworth, UNITED KINGDOM; (4) Rothamsted Research, Harpenden, UNITED KINGDOM

New technology is creating opportunities for surveillance of airborne pathogens. 'Smart' spore trapping systems collect samples referenced to parameters including GPS and climate data (temperature, wind direction, RH) and have improved compatibility with high throughput qPCR diagnostics. The Jet Spore Sampler is being tested in Australia for mobile surveillance of several significant fungal pathogens threatening the grains industry. This high frequency sampler $(450 \mathrm{~L} / \mathrm{min}$ ) collects directly into DNA extraction tubes and is mounted to a vehicle's roof-rack. It is programmed to sample automatically whenever the vehicle passes through as many as 16 pre-determined GPS polygons. This device offers improved spatial resolution of spore dispersal patterns through coordination with fixed trapping sites across a landscape. This is particularly important given the size and scale of Australia's agricultural industries. Recent work at Rothamsted Research (UK) has taken air sampling for disease forecasting a step further by development of automated spore traps with wireless reporting. This unique device is used to sample at high frequency, break open spores to release DNA at the end of the sampling period and move a sub-sample into a tube of dried reagents for either a LAMP or RPA assay. Amplified DNA of up to three different tests can be measured by fluorescence and the results sent out via mobile telemetry. It is already being used to forecast potato blight risk. These technologies aim to improve detection efficiency of rare or early influx events and provide timely warning for industry to respond to incursions of airborne plant pathogens.

\section{Dissecting the molecular cross-talk between Phytophthora-plant in the apoplastic battlefield}

Y. WANG, Nanjing Agricultural University, Nanjing, CHINA

The extracellular space constitutes an important battleground in plant-pathogen interactions. During the long run of evolution, plants have developed innate immunity to resist infection by the majority of microbes. Plants recognize microbial attack via perception of conserved microbial molecules by cell surface immune receptors and thereby operating effective defense. To achieve successful colonization, pathogenic microbes secrete effectors to modulate plant immunity. In this study, we profiled the apoplastic effector repertories secreted by the soybean root rot pathogen Phytophthora sojae using a combined proteomic and bioinformatic strategy. In this way, we identified multiple novel apoplastic effectors that are highly conserved among microbial taxa. Studies on these effectors, on the one hand reveal new tactics employed by Phytophthora pathogens to attack plant immunity, and on the other hand lead to the identification of several candidate plant receptors that regulate the recognition of apoplastic effectors. Collectively, this study contributes to the understanding of the defense and counter-defense in the plant-pathogen interactions and will be instrumental for the development of durable disease resistance resources.

Novel characteristics of Phytophthora infestans causing late blight on potato in Ethiopia D. Zeleke (1), B. Getahun (1), T. Hussien (2), C. Fininsa (2), J. YUEN (3), G. A. Forbes (4), (1) Wolaita Sodo University, Sodo, ETHIOPIA; (2) Haramaya University, Dire Dawa, ETHIOPIA; (3) Swedish University of Agricultural Sciences, Uppsala, SWEDEN; (4) International Potato Center, Servas, FRANCE

Phytophthora infestans causes late blight of potato and tomato. In this study, two distinct groups of $P$. infestans isolates are reported based on morphological and molecular characters. The isolates were recovered from 300 single lesion leaf samples collected from potato in western and southern Ethiopia. The pathogen DNA was isolated using a cetyltrimethyl-ammonium-bromide protocol. Live cultures were isolated from an actively growing lesion of 100 leaves. Mitochondrial DNA (mtDNA) analysis clearly separated the isolates into two distinct groups. One of the groups corresponds to a known $P$. infestans haplotype but the other is different with a distinct mtDNA and atypical morphological characters. Restriction fragment length polymorphism analysis established that the isolates in the latter group produced novel band patterns at two mtDNA regions. DNA sequencing of the mtDNA confirmed that the isolates represented a previously unknown variant of Phytophthora attacking Solanum tuberosum. The isolates produced papillate sporangia and chlamydospores that distinguished them from typical P. infestans isolates. All isolates were mating type A1 and the novel groups constituted a single clonal population that was genetically identical with a dominant lineage of $P$. infestans across nine microsatellite markers. This study provided new insight into a previously unknown variability within $P$. infestans in a region of the world where the pathogen population has rarely been studied.

\section{Women in Plant Pathology}

M. L. GULLINO (1), M. Mezzalama (2), (1) Agroinnova - University of Torino, Grugliasco, Torino, ITALY; (2) CIMMYT, Texcoco, MEXICO

This review will document the contributions to our discipline of the many women scientists who worked in this field since the beginning. The review will acknowledge the often obscure work of many women who contributed significantly to the advancement of plant pathology. Special attention will be given to plant pathologists in developing countries whose daily work resulted in effective plant disease management, and yields from healthy crops that increased a scarce food supply. Programmes and initiatives for attracting more women in science, with special reference to plant pathology, will be mentioned. It is the author's hope that in the near future it will not be necessary to distinguish men and women plant pathologists, but rather the contributions all who whose work is significant to the advancement of the discipline.

Fusarium wilt of banana in the Philippines: Incidence, distribution and cultivar response

F. DELACUEVA (1), F. F. M. Silva (1), A. P. Pozon (1), A. De Castro (1), V. G. Sinohin (2), T. U. Dalisay (1), D. Mostert (3), A. Viljoen (3), A. B. Molina (2), (1) University of the Philippines Los Banos, Laguna, PHILIPPINES; (2) Bioversity International, Laguna, PHILIPPINES; (3) Stellenbosch University, Stellenbosch, SOUTH AFRICA

Fusarium oxysporum f. sp. cubense (Foc) has been causing epidemics on Cavendish plantations since 1974 in the Philippines. In 2000 , a more severe and aggressive epidemic was observed which was caused by the more virulent Foc strain, the VCG 1213/16, known as Tropical Race 4 (FocTR4). Since then thousands of hectares of Cavendish plantations were destroyed by FocTR4. There is also a concern that this virulent race may affect the important local cultivars grown for the local markets in the country. A survey was carried out to determine the distribution of various Foc strains in the country, with emphasis on TR4. A total of 134 isolates were collected from 21 provinces of Luzon, Visayas and Mindanao. Foc isolates were characterized through PCR analysis using race-specific primers and analysis of vegetative compatibility groups (VCG). Results showed that Fusarium wilt is present in almost all areas surveyed but FocTR4 was detected only in Mindanao, where Cavendish bananas are grown. High incidence of Fusarium wilt was noted in Latundan, Saba and Lagkitan cultivars, but isolates from these varieties belong to Race 1 (VCG 0122 and 0123 ). Results of pathogenicity tests and field evaluation showed that three important local cultivars, namely Saba (AAA), Latundan (AAB) and Lagkitan (ABB) were all susceptible to Race 1 but 
resistant to FocTR4. Gran Naine (AAA) was resistant to Race 1 strains but susceptible to VC1213/16, the TR4 strain. Differential cultivar response to the different VCGs suggests the existence of interaction between Foc races and banana cultivars.

\section{Emerge of Xylella fastidiosa in Europe}

M. SAPONARI (1), D. Boscia (1), B. B. Landa Del Castillo (2), M. A. Jacques (3), E. Marco (4), F. Poliakoff (5), (1) CNR - Institute for Sustainable Plant Protection, Bari, ITALY; (2) Int de Agric Sostenible - SCR, Cordoba, SPAIN; (3) INRA, UMR IRHS, Beaucouze, FRANCE; (4) IVIA, Valencia, SPAIN; (5) ANSES, ANGERS Cedex 01, FRANCE

Most diseases caused by Xylella fastidiosa have been reported from North and South America. However, since 2013, widespread X. fastidiosa infections have been reported in Apulia region of southern Italy and were associated with a devastating olive disease called olive quick decline syndrome (OQDS). Localized epidemic incidence called for urgent emergency measures and surveillance programs which resulted in detection of this bacterium elsewhere in the EU, namely, Corsica and mainland France, Balearic Islands and mainland Spain. From investigations carried out, different scenarios have emerged with regard to spread and suggested that several independent introductions occured in Europe in the past years. This was indirectly confirmed by numerous interceptions of infected consignments during intensified inspections at ports of entry. The EU outbreaks have been associated with a long list of susceptible species, including several hitherto undescribed hosts and associated to new X. fastidios $a$ strains. The rapid spread of $X$. fastidios $a$ has highlighted the serious threat the bacterium poses to the EU and Mediterranean agricultural industries. The current epidemic in southern Italy is an example of the pathogen's destructive impact. Harmonized and coordinated EU research programs are underway to gain knowledge on the complex interactions of this pathogen under the EU conditions with the goal being to improve tools for early detection and control.

Calculation of dose and projected efficacy when using visible or UV light to suppress plant pathogens and arthropod pests T. MCCANN (1), D. M. Gadoury (1), A. Stensvand (2), A. Bierman (3), M. Rea (3), (1) Cornell University, Geneva, NY, USA; (2) Norwegian University of Life Sciences, Ås, NORWAY; (3) Lighting Research Center, Rensselaer Polytechnic Institute, Troy, NY, USA

Visible and ultraviolet (UV) light can be used to suppress plant pathogens and arthropod pests, but the mode of application may affect result reproducibility. Varying biological efficacy across spectra, preconditioning effects of spectra, impacts of circadian rhythm, and the question of reciprocity likely each affect biological response. The Bunsen-Roscoe law of reciprocity holds that a given dose produces an equivalent response irrespective of intensity and time. Experimental suppression of powdery mildews by UV with dose (joules) applied over a range of intensity (W/ $\left.\mathrm{m}^{2}\right)$ and time (seconds) was used to examine the impact of reciprocity on lethality. To date no study has shown that reciprocity holds at UV intensities, times, and doses previously reported. The unknown nature of reciprocity could pose problems in translating experimental data to practical treatments for both UV and visible light. We exposed conidia of $E$. necator to UVC ( $254 \mathrm{~nm}$ peak, FWHM $<5 \mathrm{~nm}$ ) doses of 0 to $200 \mathrm{~J} / \mathrm{m}^{2}$ using multiple iterations of intensity $\left(\mathrm{W} / \mathrm{m}^{2}\right)$ over times from 4 to 400 seconds; a range that encompasses many previous reports. Within this range of intensity and time, reciprocity of treatment effects held for all doses that significantly reduced conidial germination (i.e., doses from approximately 20 to $200 \mathrm{~J}$ ). Ability to reproduce results among diverse studies would be enhanced by the provision of data on spectral distribution, intensity, and duration of application for all experiments.

On the mechanisms of circulative non-propagative transmission of nanoviruses S. BLANC, INRA, Montpellier, FRANCE

Among diverse virus-insect vector interactions, the circulative non-propagative transmission has solely been reported for plant viruses. In this mode of transmission, the virus cycles within its insect vector, across cellular barriers of the gut and salivary glands, without replicating. The absence of replication is supported by the view that virus particles may undergo transcytosis at these barriers, traversing cells sequestered into membranous vesicles, thus not in direct contact with the cyctoplasm or nucleoplasm. This has been experimentally shown for the family Luteoviridae, but the mechanisms of cell crossing for the families Gemini- and Nanoviridae remain elusive. In these three families, some observations challenge the total absence of viral replication in the insect vector, denoting in the least that this mode of transmission remains poorly understood. We have initiated the study of the molecular and cellular interactions between aphid vectors and the nanovirus species Faba bean necrotic stunt virus (FBNSV). We have defined the within-vector route of this multipartite virus, where distinct viral genome segments all travel together and specifically accumulate into anterior midgut cells and into one cell type only of the principal salivary glands. Both other and our groups have shown the requirement of a "helper" factor for aphidtransmission of FBNSV. This helper factor is being identified as the product of the genome segment N, the protein NSP. At this point, we have evidence that the NSP protein controls the entry and/or accumulation of FBNSV into the aphid gut cells and further study of its mode of action is in progress.

Transcriptome analysis of cultivated and wild sweetpotato reveals differences in NB-LRR resistance gene repertoire C. H. PARADA ROJAS, L. M. Quesada, North Carolina State University, Raleigh, NC, USA

Plants possess an innate immune system with disease resistance (R) genes encoding for proteins that recognize pathogen effectors during infection. An important class of resistance gene contains nucleotide-binding and leucine-rich repeat domains NB-LRRs. Sweetpotato (Ipomoea batatas) is an important staple crop worldwide that can become infected by several pathogens in the field and postharvest. Some of these pathogens, such as Ceratocystis fimbriata, secrete effectors and can be controlled through host resistance; nonetheless, little is known about the R gene repertoire of this crop. We performed a transcriptome motif based search of NB-LRR genes in the hexaploid I. batatas (Beauregard) and the diploid wild relatives $I$. trifida (NSP306) and I. triloba (NSP323). We identified 509 NB-LRR genes in the 77,919 I. batatas transcriptome, as well as 628 and 644 NB-LRRs in the transcriptomes of I. trifida and I. triloba, respectively. Wild relatives of sweetpotato harbored more NB-LRR genes compared to cultivated sweetpotato. From the predicted NB-LRR genes in I. batatas, 56 contain an N-terminal toll/interleukin 1 receptor (TIR) domain, and 295 contain an Nterminal coiled-coil (CNL) domain. The identified NB-LRR genes provide significant insight into the resistome of sweetpotato and a first step towards identifying $R$ genes effective for control of $C$. fimbriata, the causal agent of black rot in sweetpotato.

Interactions between Diaphorina citri and 'Candidatus Liberibacter asiaticus': A systems biology perspective

M. HECK (1,2,3), E. D. A. Ammar (4), J. Bruce (5), L. Chetelat (6), S. Fattahalhosseini (2), L. A. Fleites (2), D. Hall (7), S. Hosseinzadeh (2), R. Johnson (8), S. Krasnoff (9), A. Kruse (3), M. MacCoss (10), J. S. Ramsey (9), S. Saha (2), R. Shatters (11), C. Slupsky (6), Z. Zhong (5), (1) Emerging Pests and Pathogens Research Unit, USDA-ARS, Ithaca, NY, USA; (2) Boyce Thompson Institute, Ithaca, NY, USA; (3) Section of Plant Pathology and Plant-Microbe Biology, Cornell University, Ithaca, NY, USA; (4) USDA ARS, Fort Pierce, FL, USA; (5) University of Washington, Seattle, WA, USA; (6) UC Davis, Davis, CA, USA; (7) U.S. Horticultural Research Laboratory, USDA-ARS, Fort Pierce, FL, USA; (8) Department of Genome Sciences, University of Washington, Seattle, WA, USA; (9) USDA ARS Emerging Pests and Pathogens Research Unit, Ithaca, NY, USA; (10) Department of Genomics Sciences, University of Washington, Seattle, WA, USA; (11) USDA-ARS, Fort Pierce, FL, USA 
'Candidatus Liberibacter asiaticus (CLas),' a Gram negative bacterial pathogen associated with citrus greening disease, is transmitted by Diaphorina citri in a circulative, propagative manner. Not all individuals within a $D$. citri population can become competent to transmit CLas. CLas acquisition must occur during the nymphal stage for adults to efficiently transmit. Acquisition and transmission phenotypes are heritable, and variation in CLas acquisition and transmission is found in natural $D$. citri populations. This presentation will highlight the efforts of our multidisciplinary team to use a variety of approaches, including advanced proteomics and protein interaction technologies, insect genetics, metabolomics, cell biology and biochemistry to explore the dynamic pathogen-vector interface as a function of insect genotype, infection status, developmental stage and host plant variety. Several examples of proteomics, molecular and genetic data will be discussed to illustrate the power of these approaches, when used in combination, to further our basic understanding of $D$. citri molecular responses to CLas acquisition, infection and transmission. The excellent synergism of our data with previously published studies on the biology of $D$. citri and CLas will be highlighted. Finally, intriguing parallels with other vector-plant pathogen pathosystems and the use of these approaches to develop novel targeted strategies for citrus greening control will also be presented.

International proficiency testing schemes in plant health supported by digital PCR

T. DREO, M. Pirc, N. Mehle, National Institute of Biology, Ljubljana, SLOVENIA

Proficiency tests are a way in which competence of laboratories is assessed and demonstrated. Despite them being a crucial element of quality assurance system there is a lack of providers in the field of plant health. National Institute of Biology, Slovenia is one of the few organizers and, to our knowledge, the only one to apply novel approaches for improved quality. Assignation of reference values to samples using absolute quantification of the targets with digital PCR allows for a time and cost efficient and metrologically sound preparation of samples even for non-culturable organisms e.g. 'Candidatus Liberibacter solanaceraum', phytoplasma, viruses and other. Since 2015, more than 60 participating laboratories from 27 countries and three continents took part in proficiency tests covering detection of Xylella fastidiosa, Ralstonia solanacearum, Erwinia amylovora, grapevine phytoplasmas, 'Ca . Liberibacter solanacearum', Erwinia stewartii, detection and phylotyping of Ralstonia solanacearum species complex, and fruit tree phytoplasmas. Some challenges remain, (i) international collaboration to share the load of organizing proficiency tests on alternating basis, (ii) exchange of material needed for sample preparation and (iii) an efficient transport of material via transparent and clear regulatory requirements for delivery.

\section{Mechanisms of evolution of the wheat blast fungus}

Y. TOSA (1), Y. Inoue (2,3), T. Vy (4), R. Terauchi (3,5), B. Valent (6), M. L. Farman (7), (1) Kobe Univ, Kobe, JAPAN; (2) Kobe Univ., Kobe, JAPAN; (3) Kyoto University, Kyoto, JAPAN; (4) Kobe university, Kobe, JAPAN; (5) Iwate Biotechnology Research Center, Iwate, JAPAN; (6) Kansas State University, Manhattan, KS, USA; (7) University of Kentucky, Lexington, KY, USA

Wheat blast, caused by a subgroup (Triticum pathotype) of Pyricularia oryzae, first emerged in Brazil in 1985 and spread to neighboring countries in South America. In 2016 it suddenly appeared in Bangladesh and caused a significant loss of wheat production. Here, we show how this devastating pathogen evolved in Brazil. Genetic analysis revealed that the incompatibility between Lolium isolates and common wheat is governed by an avirulence gene, $P W T 3$, and its corresponding resistance gene Rmg6 (Rwt3). We isolated PWT3 through map-based cloning. PWT3 encoded a small secreted protein composed of 141 amino acids. When $P W T 3$ of a Lolium isolate was disrupted, the resulting strain gained virulence on wheat cultivars carrying $R w t 3$. Among local landraces of common wheat collected worldwide, $R w t 3$ carriers accounted for $\sim 77 \%$ and the remaining was lacking $R w t 3$. These results indicate that mutations or deletions of $P W T 3$ would lead to a gain of virulence on a majority of wheat cultivars. Actually, wheat blast isolates had various types of mutations in PWT3, e.g. nucleotide substitutions, insertions of transposable elements into the promoter region or ORF. Historical data on wheat cultivation in Brazil suggest that the wheat blast fungus evolved due to widespread deployment of $r w t 3$ wheat followed by the loss of function of $P W T 3$. This implies that the rwt3 wheat served as a springboard for the host jump to wheat.

\section{The 'worldwide web' of forest pathogens}

B. SLIPPERS, I. Barnes, E. Steenkamp, M. J. Wingfield, Forestry and Agricultural Biotechnology Institute (FABI), University of Pretoria, Pretoria, SOUTH AFRICA

Despite the development of increasingly strict biosecurity measures during the course of the past two decades, the rate at which tree-associated fungi are moving globally continues to increase. Population genetics studies show that many of these pathogens have globally distributed genotypes and that they establish seemingly unstructured global meta-populations. These population genetics patterns can be explained only by introductions of large numbers of genotypes or multiple introductions over time. The apparent scale and frequency of these introductions could also fast track the evolution of pathogens through hybridization and admixture that might lead to unique adaptations. The diversity in this growing global pool of invasive pathogens increases their potential to overcome host resistance and environmental variation that usually restricts population growth of pathogens, and likely contributes to increasingly frequent and serious outbreaks. We have undertaken a meta-analysis of population genetics and phylogeographic studies of selected globally distributed forest pathogens in an attempt to visualize what we view as a 'worldwide web' of forest pathogens. Furthermore, we elucidate mechanisms that facilitate the movement and establishment of these pathogens. Based on these insights, we also consider the manner in which an apparent constant flow of introductions might be slowed down.

E-probes development for rapid, sensitive and specific pathogen detection in blueberries A. M. BOCSANCZY (1), A. Espindola (2), D. J. Norman (3), K. F. Cardwell (2), (1) University of Florida MREC, Apopka, FL, USA; (2) Oklahoma State University, Stillwater, OK, USA; (3) Univ of Florida MREC, Apopka, FL, USA

In 2016 Ralstonia solanacearum was isolated and confirmed on multiple blueberry farms in Florida. The PCR-based detection process for this pathogen is expensive and time-consuming. Biosecurity policy requires rule-out R3B2 select agent strains presence before plant movement, thus tests are performed in only a handful of labs nationwide. A fast, sensitive and specific diagnostic protocol would allow earlier and more accurate detection. EDNA is a bioinformatics software platform used to design specific nucleic acid E-probes, and to detect and discriminate multiple pathogens in a metagenomics database in one test. Using MiProbe tool, we designed E-probes specific to $R$. solanacearum strains associated with blueberry and we used MiDetect tool for sensitivity and specificity analyses. Simulated metagenomes were created with available blueberry and Ralstonia genomes. A percentage range of pathogen abundance $(0.001 \%$ to $10 \%)$ in the metagenome was used for the sensitivity analysis, while closely related strain genomes were added in the simulated metagenome $(10 \%)$ for the specificity test. MiDetect could detect a $0.001 \%$ pathogen abundance, while it was specific at strain level. Currently we are developing E-probes for the select agent, and we are using MinION sequencing system for double blind validation tests with plant samples. We expect to prove that EDNA coupled with MinION is a fast, sensitive, specific, and cost-effective system to detect $R$. solanacearum in blueberry. 
Characterization of endophytic bacteria with plant growth promotion and biological control potential isolated from Jatropha curcas L., a biofuel plant

P. C. Machado, P. H. M. Andrade, C. P. Sousa, P. LACAVA, Federal University of São Carlos, São Carlos, BRAZIL

The Jatropha (Jatropha curcas L.), belongs to the the family of Euphorbia. Is a plant genetically close to the Castor Plant (Ricinus communis 1.), coming from Central America and being currently distributed in all tropical regions of the globe. In Brazil, this crop has received special attention as an alternative for the supply of vegetable oil as a raw material for biodiesel manufacturing, due to the biotechnological potential of its seeds. Several studies aimed at climatic adaptations and productivity have been developed on genetic variability of $J$. curcas; but few studies have been developed focusing on the analysis of the microbial community associated with this species. The interior of the plant is inhabited by endophyte microorganisms, which can be isolated from disinfected surface and do not put on risk the plant tissues of the host plant. This group of microorganisms has the ability to stimulate the growth of plants by direct mechanisms, such as biological fixation of nitrogen, solubilization of inorganic phosphate and indole acetic acid production (IAA) and indirect mechanisms, such as the antagonism towards phytopathogens.

Potato mop-top virus TGB1 co-opts HIPP26 to activate abiotic stress signalling for long distance virus movement L. TORRANCE (1), G. Cowan (2), A. Roberts (2), S. Jones (2), P. Kumar (3), P. Hemsley (4), E. Savenkov (5), (1) James Hutton Inst, Invergowrie, UNITED KINGDOM; (2) James Hutton Institute, Invergowrie, UNITED KINGDOM; (3) School of Biology, University of St Andrews, KY16 9ST, UNITED KINGDOM; (4) Division of Plant Sciences, University of Dundee at the James Hutton Institute, Invergowrie, UNITED KINGDOM; (5) Swedish University of Agricultural Sciences, Department of Plant Biology, Uppsala, SWEDEN

Potato mop-top virus (PMTV) is soil-borne and typically occurs in potato producing regions that have cooler climates. The PMTV genome contains three overlapping open reading frames (known as a triple gene block; TGB) encoding three movement proteins that facilitate cell-to cell and long distance (systemic) movement. In PMTV the largest movement protein, TGB1, is involved in the long distance movement of both viral ribonucleoprotein complexes and virions. Previously we showed that nuclear passage of TGB1 was required for long distance movement. To gain a mechanistic understanding of the role of TGB1 we conducted a yeast two-hybrid screen to identify interacting host proteins. The screen returned 50 clones 29 of which were ranked with the highest confidence level and were all identified as $N$. benthamiana homologs of Arabidopsis HIPP26. HIPP26 is a member of the heavy metal associated isoprenylated plant protein family, a large family that contain heavy metal-binding and C-terminal isoprenylation motifs unique to vascular plants. HIPP26 is thought to act as a plasma membrane to nucleus abiotic stress signalling relay. We found that NbHIPP26 was lipid modified and localisation at the plasma membrane and plasmodesmata was mediated by lipidation. NbHIPP26 promoter-reporter fusions revealed vascular tissue specific expression with NbHIPP26 gene expression being upregulated by drought and PMTV infection. In addition, PMTV infection protected plants from drought. The data support a mechanism where interaction with TGB1 alters NbHIPP26 lipidation, releasing and redirecting membrane-associated NbHIPP26 to the nucleus thereby activating the drought stress response and facilitating virus long distance movement.

Biocontrol of fungal pathogens infecting Cannabis sativa

C. BALTHAZAR, A. Novinscak, D. L. Joly, M. Filion, Université de Moncton, Moncton, NB, CANADA

Cannabis sativa is an herbaceous plant known worldwide for its narcotic and medicinal uses (marijuana) or as a source of food and fiber (hemp). Along with proposed legislative changes to legalize cannabis in Canada for recreational consumption in 2018, great interest is now rising around organic production of $C$. sativa. However, yield and quality of $C$. sativa are affected by different diseases, many of which are caused by fungal pathogens. Biological control could be a viable alternative to the use of chemical pesticides to control fungal pathogens. In this study, a collection of 184 beneficial bacterial strains belonging to two genera (Pseudomonas and Bacillus) were tested in confrontational assays against fourteen pathogenic fungi infecting C. sativa (belonging to Botrytis sp., Sclerotinium sp., Fusarium sp., Alternaria sp., Nigrospora sp. and Phoma sp.). Two Pseudomonas and seven Bacillus strains significantly repressed the growth of most pathogens under study, suggesting wide spectrum inhibitory capabilities. Next-Gen sequencing of the genomes of these nine bacterial strains has recently been completed and comparative genomic analyses are presently underway to characterize the main genetic elements involved in biocontrol. In parallel, these bacterial strains are being further investigated under in planta conditions to validate their biocontrol efficacy.

\section{The International Plant Protection Convention (IPPC): Six decades of international cooperation for the protection of the world's plant resources}

R. L. GRIFFIN, USDA-APHIS-Plant Protection and Quarantine, Raleigh, NC, USA

The International Plant Protection Convention (IPPC) is an international treaty for cooperation in plant protection that has marked its history and impacts in three distinct periods reflecting the prevailing attitudes of governments toward regulatory plant protection. The Convention came into force in 1952 as one of many post-war initiatives designed to reduce trade friction by encouraging cooperation and shared responsibility. These ideals were reflected in the original design of the Convention, and especially the establishment of phytosanitary certification processes. The period that followed saw the IPPC increasing in complexity with a strong emphasis on technical maturity; clarifying concepts, terminology, and applications with a growing focus on trade. The last, and most recent period began with the intersection of the IPPC and the World Trade Organization Agreement of the Application of Sanitary and Phytosanitary Measures. This relationship thrust the IPPC into the fore of trade policy with responsibility for establishing international standards for phytosanitary measures applied in cross-border commerce. A major revision of the Convention accompanied organizational transformations to establish the institution we recognize today. Guidance in place and being developed by the IPPC promotes risk-based decision making toward the objective of safe trade.

Targeted Microbiome Design for Plant Health

G. BERG, TU Graz, Environmental Biotechnology, Graz, AUSTRIA

The microbiome of plants plays a crucial role in plant health. Although this is known for a long time, novel tools such as multi-omics provided novel insights into this function and their key players. Despite this progress in understanding, "a healthy microbiome" is difficult to define. The plant microbiota harbor species-specific microbial diversity consisting of bacterial, archaeal, and diverse eukaryotic species. Breeding activities resulted even in a cultivar-specific component; this will be shown for a selection of lettuce, pumpkin, oilseed rape and sugar beet cultivars. Interestingly, the breedingdriven microbiome shift is correlated with plant traits, e.g. resistance against pathogens. Microbial diversity was identified as a key factor in preventing diseases and one indication for a healthy microbiome. Therefore, it can be implemented as a biomarker in plant protection strategies. However, a critical analysis revealed that agriculture causes long-lasting anthropogenic environmental impacts and decreases biodiversity drastically. Therefore, targeted microbiome design should be used to replace the missing biodiversity, e.g. by adding key players from wild types or natural relatives. Moreover, combined breeding and biocontrol strategies maintaining diversity and ecosystem health are required. The analysis of plant microbiome data brought 
about a paradigm shift in our understanding of its role in health and disease and has substantial consequences for microbiome design and human health issues.

EDNA-Water, using deep sequencing and bioinformatics approach for water-borne plant virus detection L. PENA ZUNIGA, J. Daniels, A. Espindola, F. Ochoa Corona, Oklahoma State University, Stillwater, OK, USA

Agriculture is adversely affected by viral infections. Water-borne plant viruses: Potexvirus, Tobamovirus and Tombusvirus, have been reported worldwide and a number of them occur in the U.S. Viral infections in irrigated fields or hydroponic crops are not detected until symptoms are evident. Single-virus serological and molecular assays are used for detection. Third-generation sequencing (TGS) offers a broad detection capability to identify multiple unique Nucleic Acid signatures in metagenomic data from a single sample. Electronic probe Diagnostic Nucleic acid Analysis (EDNA) was developed to detect water-borne plant viruses in simulated mock metagenomic sequence databases (MSDs), assembled with reference positive controls and host genome sequences. MSDs were generated using MetaSim and configured to simulate Illumina average read length and error rates. Virus Eprobes ranging 20-60 nucleotides lengths were designed using EDNA MiProbe software. MSDs containing host and the target viral genome were used as positive controls. MSDs mimicking single and multiple infections were also generated. EDNA E-probes proved to be a relatively rapid diagnostic method for specific and discrete targeted viruses in water if compared with other TGS bioinformatics analyses. This provides the framework for a new sequence-based diagnosis system to make disease management decisions.

Surprises learned from plant immunity -challenges and opportunities for crop protection

Y. JIA (1), H. Zhao (2), X. Wang (3), (1) USDA ARS, Dale Bumpers National Rice Research Center, Stuttgart, AR, USA; (2) USDA ARS, 130, AR, USA; (3) UA/USDA, Stuttgart, AR, USA

Plant species have coevolved with their pathogens and both have survived under changing environments. The molecular mechanisms of disease resistance have been a focus of intense research over the last two decades. From the simplest model of elicitor and receptor interaction, to the complex active and passive defense responses learned from the plant kingdom, research has illustrated that plants have evolved remarkable multifaceted and sophisticated defense systems. These defense systems include localized and systemic responses all of which are governed by major and minor resistance $(R)$ genes. For example, rice $R$ genes that prevent infections by the fungus Magnaporthe oryzae were found among all rice chromosomes. Some blast $R$ genes and genes controlling plant productivity are co-localized on recombination suppressed chromosomal regions of the rice genome. As a result, some robust $R$ genes were lost and not effectively utilized due to domestication and extensive crop improvement efforts. Continued investigation of molecular mechanisms of plant immunity and cross-talk among genes involved in productivity can be enhanced by effective utilization of recent cutting edge technology like CRISPR-Cas 9 gene editing for crop protection. Contemporary knowledge of plant immunity and development of utilization strategies for crop improvement will be presented.

Control of cereal pathogens in the light of resistance development in Europe A. Rehfus, A. Huf, R. J. Bryson, D. Strobel, G. STAMMLER, BASF SE, Limburgerhof, GERMANY

Fungal pathogens in cereals have a significant impact on yield and quality. In wheat, Zymoseptoria tritici, Blumeria graminis f.sp. tritici, Puccinia triticina, and Fusarium spp. are important European pathogens, while in barley Pyrenophora teres, Ramularia collo-cygni and Rhynchosporium commune play a key role. Fungicides for disease control include QoIs, SBIs and SDHIs. In countries with intensive QoI use, QoI resistance conferred by the G143A exchange in the cyt $b$ has now developed in most important cereal pathogens, except for rusts, $R$. commune and $P$. teres. SBIs have been used for more than 30 years to control cereal diseases. Mutations in the cyp51 gene are responsible for sensitivity changes with the most advanced evolution of cyp51 taking place in Z. tritici, with many different haplotypes. Additionally, cyp 51 overexpression and enhanced efflux have also been detected as resistance mechanisms. An analysis of several hundred $Z$. tritici isolates from across Europe show a different distribution of cyp 51 haplotypes and cyp 51 overexpression over Europe. Despite this, SBIs are still very important tools in cereal disease control, as well as for resistance management in partnership with the highly effective SDHIs. SDHI resistance has been detected for P. teres and R. collo-cygni. Several mutations in the sdh genes cause resistance and form a complex situation in these two pathogens. $s d h$ mutations have also been found in field isolates of $Z$. tritici, but the mutation pattern and dynamic is highly different to the barley pathogens. Knowledge on fitness costs by (multiple) resistance and the effects of management strategies might help in the development of sustainable disease control strategies including also non-chemical approaches.

New approaches to detection: Canine surveillance of high risk pathogens

T. R. GOTTWALD (1), G. Poole (2), G. Mccollum (2), W. Luo PhD (3), F. Louws (4), (1) USDA-ARS, Ft Pierce, FL, USA; (2) USDA, ARS, Fort Pierce, FL, USA; (3) USDA ARS \& NCSU, Ft Pierce, FL, USA; (4) North Carolina State University, Raleigh, NC, USA

The underpinning for control of exotic diseases such as citrus Huanglongbing (HLB) caused by Candidatus Liberibacter asiaticus (CLas) and Plum Pox Virus (PPV) is early detection/response when incidence is low. Unfortunately, these pathogens can remain asymptomatic and subclinical to PCR detection for months yet can act as inoculum sources. Twenty canines were trained for early detection of HLB and two for PPV. Ten canines were each tested against 1000 trees in replicated randomized field trials with varying HLB-incidence, which resulted in 99.16\% overall detection accuracy with very few false negatives or positives. Canines also detected infected trees exclusively from 5-gm feeder root samples. In a time-course experiment, canines detected infections within 2-3 weeks of vector transmission, whereas inoculated trees were not PCR-positive for CLas until at least 3-12 mos. post inoculation. In citrus and prunus field trials, canines trot along the rows with an average interrogation time of $\sim 2-10$ trees/s; faster than any other detection method. Canines were also effectively utilized for detection of infected trees in residential areas. This confirms that canines are a very early, accurate and sensitive detection methodology. Canines are able to detect the pathogen in trees with subclinical infection, i.e., before symptom expression and considerably prior to the ability of PCR detection.

Identification of a hypervirulent pathotype of Rice yellow mottle virus: A threat to genetic resistance deployment in West-Central Africa E. HÉBRARD (1), A. Pinel-Galzi (1), A. Oludare (2), N. Poulicard (1), J. Aribi (1), S. Fabre (1), S. Issaka (3), C. Mariac (4), A. Dereeper (1), L. Albar (4), D. Silue (2), D. J. Fargette (1), (1) IRD, Cirad, Univ Montpellier, IPME, Montpellier, FRANCE; (2) AfricaRice, Bouaké, IVORY COAST; (3) FSAE, Université de Tillabéri, Tillabéri, NIGER; (4) IRD, Univ Montpellier, DIADE, Montpellier, FRANCE

Rice yellow mottle virus (RYMV) is a major biotic constraint on rice cultivation in Africa, causing high losses to rice production. Several sources of varietal high resistance are available but the emergence of virulent pathotypes that are able to overcome one or two resistance alleles can sometimes occur. Both resistance spectra and viral adaptability have to be taken into account to develop sustainable rice breeding strategies against RYMV. In this study, we assessed the adaptability of 20 viral isolates representative of the RYMV genetic and pathogenic diversity in Africa against high resistant rice 
accessions. Our results revealed a hypervirulent pathotype, named thereafter pathotype T', that is able to overcome all known sources of high resistance. This pathotype, which is spatially localized in West-Central Africa, appears to be more abundant than previously suspected. To better understand the adaptive processes of pathotype T', molecular determinants of resistance-breakdown were identified via Sanger sequencing and were validated through directed mutagenesis of an infectious clone. These analyses confirmed the key role of convergent non-synonymous substitutions in the central part of the VPg to overcome RYMV1-mediated resistance. In addition, deep-sequencing analyses revealed that virulence mutations present in a small proportion of the virus population can be sufficient for resistance-breakdown. Considering the spatial distribution of RYMV strains in Africa and their ability to overcome the RYMV resistance genes and alleles, we established a resistance-breaking risk map to optimize strategies for the deployment of sustainable and resistant rice lines in Africa.

Linking molecules with morphology in the -Omics age: Computational taxonomy pipelines for nematodes and other microbial metazoa H. BIK, University of California, Riverside, Riverside, CA, USA

Microbial metazoa (organisms $<1 \mathrm{~mm}$, including nematodes, tardigrades, platyhelminthes, other "minor" metazoan phyla, and eggs/larval stages of larger species) are abundant and ubiquitous in soil/sediment habitats, performing key functions such as nutrient cycling and sediment stability in marine and terrestrial ecosystems. Yet, their unexplored diversity represents one of the major challenges in biology and currently limits our capacity to understand, mitigate and remediate the consequences of environmental change. Microbial metazoa have a strong history of morphological taxonomy (formal species descriptions, specimen drawings, monographs, etc.), but most of this information is offline and thus effectively inaccessible for modern day -Omics studies. In addition, DNA barcoding databases and genome collections lag far behind other groups of microbial organisms such as bacteria, archaea, fungi, and single-celled protists. This sparsity of computational resources severely limits the ecological and evolutionary insights that can be gained from high-throughput sequencing approaches focused on microbial metazoa. Here, I will discuss recent efforts to improve molecular databases and expand bioinformatics pipelines for -Omic studies of "neglected" phyla such as nematodes, focusing on tool development as well as community building efforts.

\section{Surveillance for plant pests using meta-barcoding and LAMP techniques}

N. BOONHAM (1,2), T. Wood (3), J. Hodgetts (1), I. Adams (1), R. Caiazzo (3), S. Franco Ortega (4), R. Glover (1), M. Andreou (5), (1) Fera Science Ltd, York, UNITED KINGDOM; (2) Newcastle University, Newcastle upon Tyne, UNITED KINGDOM; (3) National Institute of Agricultural Botany, Cambridge, UNITED KINGDOM; (4) Agroinnova - University of Torino, Grugliasco, Torino, ITALY; (5) Optisense Ltd, Horsham, UNITED KINGDOM

The overall aim of the EMPHASIS project is to provide integrated response measures to predict, prevent and protect European agriculture, forestry sectors, and natural ecosystems from native and alien pest threats. The effectiveness of these solutions will be assessed, validated and promoted through co-innovative research and demonstration in line with end user needs and capacities. The talk will focus on the development of targeted and non-targeted methods for the detection and surveillance of quarantine pathogens and pests. LAMP tests have been developed for a range of targets in arable (including wheat, rice and potatoes), horticultural crops (including basil and lettuce) as well as diseases impacting ornamental plants and the environment (e.g. Xylella fastidiosa). The work aims to develop and validate to international standards (EPPO) a large range of LAMP assays for a number of different end users in disease diagnosis, seed testing and environmental monitoring. The assays are developed to run on the Genie suite of instruments (Optigene) and the platform has been further developed to facilitate usage, including the development of on-screen protocols, training, and formatting of kits to enhance the end-user experience. Non-targeted detection has focused on the development of meta-barcoding approaches for surveillance of airborne fungal spores and insect pests following trapping. The aim is to profile the species present in the traps and provide broad spectrum data on the presence of quarantine and other pathogens moving in a particular location. We are focusing this surveillance work on pathogens/pests of interest to farmers and regulators to facilitate the implementation of early control measures in each sector.

The mechanism of xylose-dependent expression of hrp genes in a rice pathogen Xanthomonas oryzae pv. oryzae Y. IKAWA, S. Tsuge PhD, Kyoto Prefectural University, Sakyo-Ku, Kyoto, JAPAN

hrp genes encoding components of the type III secretion system are essential for virulence of Xanthomonas oryzae pv. oryzae (Xoo), the bacterial pathogen of leaf blight of rice. Expression of $h r p$ genes is regulated by two key regulators, HrpG and HrpX. Previously, we reported the importance of xylose in $h r p$ gene expression in the bacterium, and that, without xylose, the accumulation of a hrp regulator, HrpX, not the expression of the protein, is decreased. Here, to clarify the mechanism of quantitative regulation of HrpX, we conducted random transposon mutagenesis, and found that a mutant with a transposon in a LacI-type transcriptional regulator XylR showed high $h r p$ gene expression even under the xylose-free medium. The $x y l R$ deletion mutant showed high HrpX accumulation and hrp gene expression in the medium without xylose, similarly to the wild type incubated in the xylosecontaining medium. XylR is known to negatively regulate xylan/xylose metabolism-related genes, including a xylose isomerase gene $x y l A 2$, in other bacteria. We confirmed the involvement of Xoo XylR in the negative regulation of $x y l A 2$ and, furthermore, the binding of the protein to the upstream region of the xylA2. These results suggest that, in the presence of xylose, inactivation of XylR activates xylan/xylose utilization and, simultaneously, of hrp gene expression through the higher accumulation of HrpX in Xoo. The mechanism of the XylR-dependent quantitative regulation of HrpX will be discussed.

Quality assurance, validation of tests and collections in plant pest diagnostics: Approaches and experience in the EPPO region F. PETTER, M. McMullen, B. Giovani, European and Mediterranean Plant Protection Organization, Paris, FRANCE

EPPO is an intergovernmental organization responsible for European cooperation in plant health. The EPPO diagnostic programme started in 1998 with the preparation of pest specific diagnostic protocols. Development of quality management systems and accreditation quickly became a concern for many laboratories in the EPPO region. EPPO Standards have been approved on quality assurance providing specific guidance for plant pest diagnostics (how to perform the validation of tests, how to organize interlaboratory comparisons...). Standards are regularly revised to update them with most recently developed approaches in accreditation and validation. Laboratories in the EPPO region are increasingly working under quality assurance systems and the need for laboratories to have access to well characterised biological reference material for morphological identification and for the use, development and validation of tests has been recognized. EPPO participated in an EU FP7 project on collections (Q-collect) which resulted in the development of a white paper on collections. Finally, to increase active collaboration among the Organizations involved in plant health research activities at national and regional levels, Euphresco (European Phytosanitary Research Coordination) was established to favour synergies among national research activities and to support plant health policy. Euphresco members identify research priorities to be tackled through transnational collaboration. Many research projects have been funded including projects to develop new tests, validate these tests or evaluate the proficiency of laboratories. These approaches and the experiences in the EPPO region will be presented. 
Anticipating and understanding new Xylella fastidiosa epidemics across European landscapes; insights from remote sensing and network analysis

P. S. A. BECK (1), C. Camino González (2), R. Calderón Madrid (2), A. Hornero Luque (2), R. Hernández-Clemente (3), T. Kattenborn (4), M. Montes Borrego (2), D. Susca (5), M. Morelli (6), V. González (2), P. North (3), C. J. Carstens (7), B. B. Landa (2), D. Boscia (6), M. Saponari (6), G. Strona (1), J. A. Navas-Cortes (2), P. J. Zarco-Tejada (1), (1) European Commission - Joint Research Centre, Ispra, ITALY; (2) Instituto de Agricultura Sostenible, Consejo Superior de Investigaciones Científicas, Cordoba, SPAIN; (3) Swansea University, Swansea, UNITED KINGDOM; (4) Karlsruher Institut für Technologie (KIT), Karlsruhe, GERMANY; (5) Università di Bari Aldo Moro, Bari, ITALY; (6) CNR - Institute for Sustainable Plant Protection, Bari, ITALY; (7) School of Mathematical and Geospatial Sciences, RMIT University, Melbourne, AUSTRALIA

Europe's major $X$. fastidiosa outbreaks have progressed steadily in the past years as data on the bacterial strains causing them, and on the host range and vectors of the pathogen in various regions, became available. The initial uncertainty around these critical epidemiological aspects of the $X$. fastidiosa invasions hampered estimates of their rate and reach should eradication or containment efforts fail. We explored whether network analysis could help circumvent known knowledge gaps in X. fastidiosa epidemiology; Relying only on distribution maps of a known host plant ( $>60000$ olive groves), and the dispersal abilities of a putative vector, we gained network-based insights into the effort required for eradication or containment of the pathogen in southern Italy, and into opportunities to optimize pest management efforts at the landscapes scale. Early detection of new foci play a critical role in ensuring such efforts are efficient. Hyperspectral and thermal remote sensing, at spatial resolutions fine enough to delineate tree crowns, provide estimates of several plant functional traits in individual trees. After coordinated aircraft and field campaigns, we found that based on these traits, olive trees with $X$. fastidiosa infection symptoms can be detected and mapped with $>80 \%$ accuracy. Furthermore, we found strong evidence that they, in some cases, allow the identification of $X$. fastidios $a$ affected trees weeks to months before they developed visible symptoms.

Analyzing the expression of Candidatus Liberibacter solanacearum effectors in insect and plant hosts

P. REYES CALDAS, L. M. M. Perilla Henao, S. Thapa, C. Casteel, G. L. Coaker, University of California, Davis, CA, USA

Candidatus Liberibacter solanacearum (Lso), the causal agent of Zebra Chip disease, is an emerging pathogen causing significant crop losses in Solanaceous plants. There are no commercial cultivars with resistance to Zebra Chip, making disease management difficult. Lso haplotypes A and B are transmitted by the psyllid vector Bactericera cockerelli and infect the phloem of tomato and potato. Many bacterial pathogens secrete effectors to manipulate and establish in their hosts. Genome analyses revealed the presence of the SEC secretion system in Lso. We utilized available genome sequences to predict conserved SEC-dependent effectors from haplotype A (R1, Lso-NZ1, HenneA), haplotype B (CLso-Z1), and haplotype C (FIN111, FIN114). Effectors were predicted according to the presence of the secretory signal peptide using SignalP 3.0 and 4.0, proteins smaller than $25 \mathrm{KDa}$ without a transmembrane domain were selected and manually verified. We identified a suite of core effectors present in haplotypes infecting tomato and potato. The expression of core Lso effectors was analyzed in plant and insect hosts by qPCR, identifying those that are preferentially expressed in either organism. Lso effector expression patterns could be used to predict the role of individual proteins in colonizing the insect vector and plant host. A greater understanding of how phloem-limited bacterial pathogens interact with their hosts can ultimately be used to develop targeted disease control strategies.

Dream big: Solid state/LED lighting will allow you to affect pathogen biology in ways that you never could before M. REA (1), D. M. Gadoury (2), (1) Lighting Research Center, Rensselaer Polytechnic Institute, Troy, NY, USA; (2) Cornell University, Geneva, NY, USA

Plants and pathogens coevolved over millions of years in an environment of $24 \mathrm{hr}$ cycles of natural sunlight during day, and near-complete darkness at night. By comparison, horticultural uses of electric lighting span a mere instant of evolutionary time: less than 100 yr. High Pressure Sodium (HPS) represents the most common electric lighting source used for enhanced plant growth today, but Light Emitting Diode (LED) technologies have been developed to the point where they are durable and energy efficient, albeit at a 2- to 6-fold higher purchase price. An under-exploited benefit of LEDs is their ability to be precisely controlled in terms of spectrum, amount, duration, timing, and distribution for the purpose of suppressing plant pathogens, while still providing enhanced plant growth. LEDs make possible the strategic control of the lighting environment to attack photoregulatory pathways of pathogens, and thereby disrupt pathogenesis. A number of examples will be presented, encompassing suppression of downy mildews, disruption of circadian rhythms, stimulation of inoculum release during environmentally unfavorable periods, effects of selected wavelengths on the sporulation process, and potential effects of counter-phasing of different spectra. The unique physical and electromechanical attributes of LED systems as they relate to these potential uses, their limitations, and directions for future research will be discussed.

An effector from the Huanglongbing-associated bacterium targets a specific family of proteases in citrus K. CLARK (1), S. Schwizer (1), Z. Pang (2), E. Hawara (1), J. Franco (3), D. Pagliaccia (1), L. Zeng (1), G. L. Coaker (4), N. Wang (2), W. Ma (1), (1) University of California, Riverside, Riverside, CA, USA; (2) University of Florida, Lake Alfred, FL, USA; (3) University of California, Davis, Davis, CA, USA; (4) University of California, Davis, CA, USA

The citrus industry continues to be threaten by Huanglongbing (HLB) disease. All citrus cultivars can be affected by the HLB-associated bacterium, Candidatus Liberibacter asiaticus (CLas), and effective management strategies are still needed. Insect-transmitted and phloem-colonizing pathogens, like CLas, utilize the general Sec secretion system to deliver virulence proteins into the host. These proteins can aid infection by manipulating plant physiology and subverting host immunity, thereby promoting bacterial colonization and disease progression. We investigated the virulence function of a $C$ Las effector, Sec-delivered effector 1 (SDE1), which is conserved among all CLas isolates. SDE1 has $\sim 10$-folds higher expression in plant hosts compared to the insect vector, implicating a possible role in HLB progression. Using yeast two-hybrid screening, we found that SDE1 directly interacts with several members belonging to a specific family of proteases, known as papain-like cysteine proteases (PLCPs). PLCPs in other pathosystems have been shown to contribute to plant defense against pathogens including bacteria, fungi and Phytophthora. Interestingly, all these different pathogens produce effectors to inhibit PLCP activity and promote infection. We found that SDE1 also inhibits the protease activity of PLCPs in vitro and in citrus. Using a surrogate system, we further showed that SDE1 can promote bacterial infection. These findings support a model that SDE1 acts as a PLCP inhibitor in order to suppress immune response in citrus and promote $C$ Las infection. We will discuss ongoing progress on the characterization of SDE1 and the potential of exploiting SDE1-PLCP interaction for the development of HLB management.

Grapevine and Fungal Trunk Pathogens Interactions and the Global Impacts of Climatic Events F. FONTAINE, Université de Reims Champagne Ardenne, Reims, FRANCE

Grapevine trunk diseases (GTDs) represent one of the most important problems for viticulture worldwide. Characterizing the impact of GTDs on grapevine physiology is a key step to understanding the mechanisms that lead to disease development and symptoms expression, which will assist in the development of effective control strategies against GTDs. For GTD-affected vines, carbon metabolism seems to be strongly altered associated with an 
induction of stress/defence responses. Detoxification processes are also affected; which could be linked to the production of extracellular compounds by GTD pathogens some of which are phytotoxic. These processes tend to strengthen the plant cell wall, maintain the osmotic and redox balance, destroy the fungal cell walls and battle against fungi. The production of phytotoxins by GTD pathogens is modulated by environmental factors such as temperature and water stress and depends on the fungal strains and the vine cultivar and rootstock. In addition to the strong relationship between the grapevine and the GTD pathogens, the microbiota, including both beneficial and pathogen microorganisms needs to be taken into consideration. The balance and the diversity of the microbiota could interfere in the virulence of fungi and vine physiology. All these relationships still remain unclear and need to be deciphered to better understand the emergence of the GTD foliar symptoms.

Global impacts of potential plant disease epidemics: Wheat and Rice

S. S. SAVARY, INRA, Castanet Tolosan, FRANCE

The current challenges in feeding today's world population can be examined in view of our understanding of the evolution of food production in history, from the Neolithic Revolutions, to the Green Revolution, to the present. Cereals, especially rice and wheat, play a pre-eminent role in ensuring global food security. These two crops are exposed to a number of grave plant diseases, which may in turn threaten global food security. These diseases may be associated with three broad, non-exclusive, epidemiological patterns: chronic, acute, or emerging. These three epidemiological patterns can affect differently the several components of food security, from production, to access, and to safety. The global impacts of plant diseases can be assessed through successive steps, including the use of epidemiological models to simulate potential epidemics, and the use of crop growth models including damage mechanisms to simulate yield losses. Scenario development and analysis constitute a powerful framework of investigation, since the inputs of these models can be driven by global change scenarios, which incorporate climate change scenarios. Analysing the impacts of plant disease epidemics has a range of applications, including the design of research strategies, the evaluation and guidance of policies, and improved management of plant diseases.

Characterization of two biologically distinct variants of Tomato spotted wilt virus

R. O. ADEGBOLA (1), S. Jarugula (1), S. Marshall (1), S. Adkins (2), R. Naidu (3), (1) Washington State University, Prosser, WA, USA; (2) USDA, ARS, US Horticultural Research Laboratory, Fort Pierce, FL, USA; (3) Washington State University - IAREC, Prosser, WA, USA

Tomato spotted wilt virus (TSWV, genus: Orthotospovirus; family: Tospoviridae) causes significant economic losses to a wide range of crops. In this study, one TSWV isolate from basil (TSWV-C) and a second isolate from tomato (TSWV-Wa1) were established in Emilia sonchifolia. Time-course analysis of inoculated $E$. sonchifolia plants by ELISA using $\mathrm{G}_{\mathrm{N}^{-}}, \mathrm{G}_{\mathrm{C}^{-}}$and NSs-specific antibodies revealed similar amounts of the $\mathrm{G}_{\mathrm{N}}$ and $\mathrm{G}_{\mathrm{C}}$ glycoproteins present in plants infected with TSWV-C and TSWV-Wa1 isolates, but higher amounts of NSs in TSWV-C-infected plants. In host range studies, the two isolates showed distinct phenotypic differences with TSWV-C producing severe symptoms on several permissible hosts compared to TSWV-Wa1 that showed limited host range. TSWV-C caused systemic infection with severe symptoms in a susceptible tomato cultivar compared to TSWV-Wa1 that was found to be largely localized to inoculated leaves. The S-, M-, and L-RNA sequences of the two distinct isolates, generated using a combination of Sanger sequencing and high-throughput sequencing, showed 96.6 to $97.9 \%$ identity at the nucleotide level. Among the different ORFs, the NSs gene was more divergent, with $97.0 \%$ identity at the amino acid level. In agroinfiltration assays, the TSWV-Wa1 NSs protein showed weaker RNA silencing suppression (RSS) activity than the TSWV-C NSs protein. Specific amino acid substitutions were found responsible for the observed weak RSS activity of the TSWV-Wa1 NSs protein.

Integration of DNA-based diagnostics with air samplers in a country-wide sampling network for plant disease forecasting M. JEDRYCZKA, J. Kaczmarek, W. Irzykowski, P. Serbiak, Institute of Plant Genetics, Polish Academy of Sciences, Poznan, POLAND

The dispersal of spores of fungal plant pathogens can be nowadays studied with various techniques, primarily with volumetric air samplers. These devices help to identify the pathogens present in the spore form in certain areas and time. Additionally, they allow to measure their quantity, which enables comparison of values between different sites. However, the spore counts are time and labour consuming and the similarity of spores across fungal species or even numerous fungal genera, makes these comparisons and calculations ambiguous or even unreliable. Recent advances in the DNAbased diagnostics combined with spore sampling help to fill this gap. Novel techniques, such as qPCR allow precise identification of pathogens.

Moreover, they allow to identify not only the exact genus and species, but also subspecies, race, special form, chemotype or a form which is resistant to certain chemical compound present in fungicides, provided a genetic modification of this form (deletion, SNP, indel, mutation) has been previously recognized. There are several examples of successful identification of genetic variants present in spores bearing certain virulence genes, that can break the resistance of susceptible plant cultivars. In Poland, DNA-based diagnostics combined with the spore capture by a network of air samplers has been successfully used since 2004 in a country-wide disease forecasting of stem canker of oilseed rape (www.spec.edu.pl) and, to some extent, in Fusarium Head Blight (www.fusar.edu.pl). Moreover, such solutions can be also used nowadays in modern allergology to inspect the sources of spores carrying compounds that can cause inhalatory allergies.

\section{A 'cool' mechanism of salicylic acid-mediated defense and growth tradeoff} C. J. TSAI, University of Georgia, Athens, GA, USA

Crop protection based on induced resistance via treatment with salicylic acid (SA) or SA analogs offers the prospect of broad-spectrum disease control, but often comes with yield loss due to as yet poorly understood mechanisms. Mechanistic investigation of SA inhibition on plant growth has been challenging, in part because many SA-elevated Arabidopsis mutants are perturbed in immune signaling pathways with pleiotropic effects. We generated a novel suite of transgenic Arabidopsis that hyperaccumulates SA and SA-conjugates, not because of a mutation, but by ectopic expression of a bacterial SA synthase. These "biosynthetic mutants" exhibited enhanced disease resistance to Pseudomonas syringae pv. tomato DC3000, and enhanced tolerance to salinity and osmotic stresses. Plant growth was inhibited in an SA-dependent manner, and the effect was exacerbated at cool temperatures. Transcriptomics analysis revealed an inhibitory effect of SA on a group of cold-regulated (COR) genes implicated in membrane protection against abiotic stresses. Constitutive expression of COR genes rescued the growth phenotype in high-SA plants without affecting disease resistance. Elucidating the growth-immunity-temperature interplay promises to improve crop protection strategies that deploy SA-based disease resistance without yield penalty under fluctuating environments.

Resource competition and antagonism in natural soil suppressive to Bayoud disease on date palm (Phoenix dactylifera) in Morocco A. ESSARIOUI (1), N. LeBlanc (2), D. C. Schlatter (3), J. Anderson (4), L. K. Otto-Hanson (5), H. C. Kistler (5), L. L. Kinkel (6), (1) INRA, Errachidia, MOROCCO; (2) USDA-ARS, Beltsville, MD, USA; (3) USDA ARS, Pullman, WA, USA; (4) Department of plant pathology/University of 
Minnesota, St Paul, MN, USA; (5) Department of Plant Pathology/University of Minnesota, St Paul, MN, USA; (6) Department of Plant Pathology/University of Minnesota, Saint Paul, MN, USA

Date palm cultivation in Morocco suffers from Bayoud, a vascular disease caused by the soilborne fungus Fusarium oxysporum f. sp. albedinis. Some soils are naturally suppressive to the disease, but we have limited knowledge of how this occurs. To improve our understanding of mechanisms by which Bayoud is suppressed, we characterized functional traits of Fusarium isolates and the composition of bacterial and fungal communities in one suppressive and two conducive soils. Thirty saprophytic Fusarium isolates were randomly selected from each soil sample. In vitro inhibitory activity of every isolate against the Bayoud pathogen was assessed and isolate growth on 95 carbon substrates was evaluated using Biolog plates. The composition and diversity of fungal and bacterial communities were analyzed in each soil sample by sequencing the internal subscribed spacer (ITS1) and the ribosomal (16S) genes, respectively. Fusarium isolates from one conducive soil had the greatest antagonistic activity, but the slowest growth, suggesting tradeoffs between inhibition and growth. However, Fusarium isolates from the second conducive soil showed the lowest frequency and intensity of antagonism with growth efficiency that was comparable to that of the pathogen. Isolates from the suppressive soil displayed intermediate antagonism but the greatest growth. Sequence data revealed greater microbial diversity in the suppressive compared to the conducive soils with enrichment in microbial taxa known for their prolific production of antimicrobial compounds including Fusarium, Aspergillus, and diverse Actinomycetes. Microbial antagonism and resource competition may jointly contribute to Bayoud disease suppression in the suppressive soils in Morocco.

Overcoming barriers: Dikes, policy, tulips, religion and the Dutch success in agriculture production and trade P. BOONEKAMP (1), J. Horsten (2), (1) Royal Netherlands Society of Plant Pathology, Wageningen, NETHERLANDS; (2) Royal Netherlands Scociety of Plant Pathology, Wageningen, NETHERLANDS

'A tiny country feeds the world' was the title of an extensive article in the National Geographic of September 2017. As representatives of the KNPV, founded in 1891 and the oldest phytopathology society in the world, we would like to present the historical context. Historical factors related to 'overcoming barriers' are discussed. With half of the country below sea-level, the Dutch started in the $13^{\text {th }}$ century building dikes to acclaim fertile land from sea, lakes and marshes. The necessary egalitarian governing system to control the Polders, led to the first republic of the world in the $16^{\text {th }} / 17^{\text {th }}$ century, governed by citizens and not by nobility. The freedom of religion attracted foreign refugees who brought in knowledge and money to boost the economy, and to promote trade all over the world, but also stimulated literacy among all levels of society. These historical factors lead to a perfect birthground for well-educated farmers producing exotic vegetables and flowers (e.g. potato, tomato, tulips) in the $17^{\text {th }} / 18^{\text {th }}$ century. When mid- $19^{\text {th }}$ century plant diseases got a scientific basis the Dutch belonged to the first to set up scientific institutes and to connect them with the farming practice in order to maintain the high quality standard of domestic but especially of the exported food products. The close collaboration between science, government and agro-entrepreneurs - the 'golden Triangle' - in the $20^{\text {th }}$ century shaped The Netherlands into 'A tiny country feeding the world' in the $21^{\text {st }}$ century.

The Pectobacterium complex: Diversity and phylogeny

K. Chawki (1), A. Quêtu-Laurent (1), G. Taghouti (2), E. Caullireau (2), M. Fischer-Le Saux (2), Y. Le Hingrat (3), D. Andrivon (4), P. Portier (2), V. HÉLIAS (1), (1) French Federation of Seed Potato Growers, Le Rheu, FRANCE; (2) National Institute for Agronomic Research, Beaucouzé, FRANCE; (3) French Federation of Seed Potato Growers, Paris, FRANCE; (4) National Institute for Agronomic Research, Le Rheu, FRANCE

Pectobacterium is one of the two bacterial genera responsible for blackleg and tuber soft rot on potato. Pectobacterium contains today eight species, namely $P$. atrosepticum, $P$. carotovorum, $P$. betavasculorum, $P$. wasabiae, $P$. parmentieri, $P$. polaris, $P$. cacticida, and $P$. aroidearum after recent new assignments. Field surveys conducted annually in France since 2003, coupled with diversity studies of the resulting strain collections, showed that blackleg symptoms are associated with a much larger range of species than the one ( $P$. atrosepticum) historically almost exclusively held responsible for these symptoms in Europe. We thus aimed at analyzing more deeply the diversity and the phylogeny of Pectobacterium, particularly strains isolated from field symptoms on potato, and at evaluating changes within the P. carotovorum (P.c.) group during the last decades. Refining the taxonomy of the Pectobacterium groups was needed to develop specific detection tools, useful for testing and certifying seed lots. A MLSA was performed using recA, $d n a X$ and leuS genes on 384 strains from the FN3PT and the CIRM-CFBP collections; 53 Pectobacterium sequences from databases were used as supplementary data. The already described species including the recently described P. polaris and the subspecies $P$. c. odoriferum, $P$. c. brasiliense, $P$. c . actinidiae were clearly defined by the MLSA results. In contrast, strains from $P$. carotovorum subsp. carotovorum showed a high genetic diversity in accordance with the earlier sequencing work on PelY. Our results showed that $P$. carotovorum subsp. carotovorum strains were distributed in several groups, all not being previously described.

Xylella fastidiosa evolution, determinants of host plant specificity, and pathogen adaptation to novel hosts R. P. P. ALMEIDA PHD, University of California Berkeley, Berkeley, CA, USA

Despite the global importance of the bacterium Xylella fastidiosa, there is limited understanding of what determines its host specificity, and how this pathogen adapts to new host plant species. Analyses of genomic data for $X$. fastidiosa populations in the USA and Europe will be presented as case studies, and hypotheses generated based on these datasets discussed.

\section{Understanding the function of a novel Gr29D09 effector family from the potato cyst nematode Globodera rostochiensis in host defense} suppression

A. Y. C. YEH (1), S. Chen (1), T. T. Tran (1), T. J. Baum (2), X. Wang (3), (1) School of Integrative Plant Science, Cornell University, Ithaca, NY, USA; (2) Department of Plant Pathology and Microbiology, Iowa State University, Ames, IA, USA; (3) USDA-ARS, Robert W. Holley Center for Agriculture and Health, Ithaca, NY, USA

Potato cyst nematodes (PCNs) Globodera rostochiensis and G. pallida are among the most devastating pests of potatoes causing substantial yield losses worldwide. To enable successful infection, PCNs secrete a repertoire of effector proteins into root cells, which are subsequently transformed into a complex feeding structure that sustains nematode growth. The 29D09 effector family, which contains multiple members, is present in both Globodera and Heterodera spp. cyst nematode species. Our previous study showed that Gr29D09 effectors from G. rostochiensis play an important role in host defense suppression. Using affinity purification of protein complexes extracted from nematode-infected potato roots overexpressing the Gr29D09-GFP fusion protein followed by mass spectrometry analysis, we obtained a list of candidate host interacting proteins including hexokinase 7 (StHXK7). Furthermore, both co-immunoprecipitation and bimolecular fluorescence complementation assays demonstrated that Gr29D09 effectors can interact with StHXK7 in planta. Interestingly, a subset of $H X K$ and $H X K$-like genes were found to be upregulated in PCN-infected potato roots as well as in Heterodera schachtii (sugar beet cyst nematode)-infected Arabidopsis roots. We are currently using the Arabidopsis-H. schachtii pathosystem to further investigate the defense pathways that may be regulated by the 29D09 effector family. 
Multiscale aspects of vector transmission of plant viruses: From landscapes to coinfections

A. POWER, Cornell University, Ithaca, NY, USA

Cornell University, Ithaca, NY, USA

Potato virus $Y$ (PVY) is one of the most serious pathogens of potato crops globally, reducing both yield and quality. Highly damaging recombinant virus strains have emerged in recent decades, adapted rapidly to potato ecosystems, and dominated virus populations over extensive geographical areas. Our research is focused on understanding the ecological factors that allow recombinant virus strains to emerge, spread rapidly, and become dominant. We find that landscape composition and structure, vector community composition, transmission dynamics, and within-host processes all influence the epidemiology of both the ordinary strain $\left(\mathrm{PVY}^{\mathrm{O}}\right)$ and recombinant strains such as PVY ${ }^{\mathrm{NTN}}$. At the landscape scale, recombinant strains and overall PVY incidence are positively associated with row crops in the landscape around a farm and negatively associated with forested habitats. PVY incidence is positively correlated with landscape fragmentation and total edge between habitat types, but negatively correlated with higher diversity of habitat types in the landscape. Vector abundance and species richness also vary significantly with landscape composition. At the host scale, recombinant viruses typically dominate in mixed infections and are more successful in vertical transmission through infected tubers. Taken together, these findings improve our understanding of the widespread dominance of recombinant PVY strains.

A systematic approach for developing molecular markers for Oomycetes

F. N. MARTIN, USDA ARS, Salinas, CA, USA

Having a systematic approach for designing amplification primers for genes that are useful for isolate identification $\&$ phylogenetic studies, population studies, diagnostic assays, and metagenomic studies would enhance research opportunities. This is particularly true for Oomycetes due to the difficulty in morphology-based species classification and the obligate nature of some taxa. To address this need, the mitochondrial genomes of over 500 isolates representing 155 taxa for a range of Oomycetes have been assembled and comparative genomics conducted. Gene order differences compared to plant and Eumycotan fungal genomes have been useful for development of specific primers for amplification of genes ( $r p s$ 10) from a wide range of taxa (including downy mildews) and design of diagnostic assays. A single assay capable of detecting Phytophthora at a genus and species-specific level has been validated with data supporting development of species-specific TaqMan probes for $89 \%$ of the genus. Similar types of assays are in development for Pythium, Aphanomyces and several downy mildews. Unique putative open reading frames also are useful for design of species-specific diagnostic assays for pathogen detection and quantification (Bremia lactucae). Mitochondrial genome assembly for multiple isolates of the same species has identified loci useful for haplotype classification in population studies, and some of the loci noted above are also useful in metagenomics studies.

Whitefly-transmitted viruses induce contrasting changes in vector behavior and plant volatile emissions A. FERERES, CSIC, Madrid, SPAIN

Virus infection frequently modifies plant physiology, leading to changes in behaviour and performance of their insect vectors in a way that transmission is enhanced, although this may not always be the case. Here, we investigated Bemisia tabaci response to tomato plants infected by Tomato chlorosis virus (ToCV), a non-circulative-transmitted crinivirus, and Tomato severe rugose virus (ToSRV), a circulative-transmitted begomovirus. Moreover, we examined the role of visual and olfactory cues in host plant selection by both viruliferous and non-viruliferous whiteflies. Visual cues alone were assessed as targets for whitefly landing by placing leaves underneath a Plexiglas plate. Thereafter, we tested the whitefly response to volatiles using an active air-flow Y-tube olfactometer, and chemically characterized the blends using gas chromatography coupled to mass spectrometry. Visual stimuli tests showed that whiteflies, irrespective of their infectious status, always preferred to land on virus-infected leaves. ToSRV-infection promoted a sharp decline in the concentration of some tomato volatiles, while an increase in the emission of some terpenes after ToCV infection was found. ToSRVviruliferous whiteflies preferred volatiles emitted from mock-inoculated plants, a conducive behaviour to enhance virus spread, while volatiles from ToCV-infected plants were avoided by non-viruliferous whiteflies, a behaviour that is likely detrimental to the secondary spread of the virus.

Application of spectral reflectance to differentiate between leaf diseases and abiotic stresses in wheat A. FICKE (1), K. Kusnierek (2), (1) NIBIO, 1430 Aas, NORWAY; (2) NIBIO, Kapp, NORWAY

The transition from the uniform application of fertilizers and fungicides to site-specific management requires accurate measurement and differentiation of the stresses involved in predicted yield reduction. Our study was designed to show how automated non-contact sensor technology, based on spectral reflectance, can be implemented to differentiate between diseases, nitrogen and water stress. We designed field trials with three nitrogen levels, three irrigation conditions and two fungicide treatments, to obtain a range of different biotic and abiotic stresses important for yield. We then obtained subsamples of leaves at GS 60, with and without diseases (septoria nodorum blotch, yellow rust and powdery mildew) in combination with different water and nitrogen conditions. The ASD FieldSpec 3 spectrophotoradiometer was used to take images under controlled light conditions to determine the energy spectra emitted from each leaf surface. Utilizing multivariate statistics, we related the variance in reflectance to the determining factors involved. Disease severity was assessed at GS 70-75 in the field and yield determined. The Principal Component Analysis (PCA) model developed from the analyzed images described over $90 \%$ of the total variance in the five first components, with each of the different stresses being clearly separated. Analysis of the yield determining factors for all three years showed that year, nitrogen, water, disease and year X disease were all significant.

Emergence of a resistance breaking TSWV strain in tomato in California

O. BATUMAN (1), M. Rojas (2), M. Macedo (2), S. Adkins (3), R. L. Gilbertson (4), (1) University of Florida IFAS, Immokalee, FL, USA; (2) University of California Davis, DAVIS, CA, USA; (3) USDA, ARS, US Horticultural Research Laboratory, Fort Pierce, FL, USA; (4) Department of Plant Pathology, University of California, Davis, CA, USA

Tomato spotted wilt virus (TSWV) is a highly destructive pathogen of tomato in the Central Valley of California, USA. During the 2016 tomato growing season, unusually early and severe symptoms of TSWV occurred in fields of TSWV-resistant (Sw-5) fresh market tomato cultivars in Fresno County. High disease incidences (50-80\%) were observed in some fields. The result of biological and molecular (sequence) analyses of TSWV isolates from these symptomatic Sw-5 tomato plants collectively revealed that these infections were caused by a newly emerging resistance-breaking (RB) TSWV strain in California. Furthermore, in mechanical inoculation assays in a greenhouse, it was established that RB TSWV strains infected fresh market and processing tomato cultivars with and without the Sw-5 gene. The emergence of RB TSWV strains in California occurred 5-6 years after ever-increasing planting of Sw-5 cultivars. The widespread use of Sw-5 cultivars, especially in 'hotspot' areas, is an important component of an effective integrated pest management (IPM) strategy, which was developed in California and helped reduce TSWV incidences in processing tomatoes for over a decade now. The new RB strain appears to have evolved following a mutation, which was associated with the C118Y substitution in the NSm gene of a local wild-type TSWV strain. With the emergence of the RB strain, the IPM program needs to be revisited and revised. 
Rice stripe virus interferes with S-acylation of remorin and induces its autophagic degradation to facilitate virus infection X. ZHOU, Institute of Plant Protection, CAAS, China, Beijing, CHINA

Remorins are plant-specific membrane-associated proteins, and were proposed to play crucial roles in plant-pathogen interactions. However, little is known about pathogens' counter-response to remorin-mediated regulation. Here, we find the remorin protein (NbREM1) is down-regulated early in Rice stripe virus (RSV) infection by quantitative whole-proteome analysis. We demonstrate that the turnover of NbREM1 is regulated by the S-acylation modification and can enter the autophagy pathway for degradation. We further show that RSV can interfere with the S-acylation of NbREM1, that is required for negatively regulates RSV infection by restricting virus cell-to-cell trafficking. The interference of NbREM1 S-acylation affects the targeting of NbREM1 to the plasma membrane microdomain, and the resulting accumulation of non-targeted NbREM1 is subjected to the autophagic degradation, causing down-regulation of NbREM1. In addition, we find the RSV-encoded NSvc4 protein alone can interfere with NbREM1 S-acylation through binding with the C-terminal domain of NbREM1. Moreover, we reveal a same counter-response of NSvc4 to the homologous remorin (OsREM1.4) from Oryza sativa, the original host of RSV, indicating down-regulating remorin protein level by interfering its S-acylation is a common strategy that RSV has adopted to overcome remorin mediated inhibition of virus movement.

Detection and classification of Candidatus Phytopalsma associated with cassava witches' broom disease in Thailand P. MOONJUNTHA (1,2), P. Maneechoat (3), N. Kositcharoenkul (3), P. Wongtiem (2), K. T. Natsuaki (1), (1) Tokyo university of Agriculture, Tokyo, JAPAN; (2) Rayong Field Crops Research Center Department of Agriculture, Rayong, THAILAND; (3) Plant Pathology Research Group Department of Agriculture, Bangkok, THAILAND

Cassava witches' broom (CWB) diseases have been reported in Viet Nam since 2013 with phytoplasma in 16SrI and 16SrII groups and in Thailand since 2017 in 16SrI group. The CWB shows symptoms such as small and yellowing leaves, leaf proliferation and stem necrosis. In Thailand CWB like symptoms are observed in many cassava planted area. To detect phytoplasma associated with CWB in Thailand, $65 \mathrm{CWB}$ samples were collected from 156 fields in 11 provinces in Thailand during year 2015 - 2016. Nested PCR by two sets of primer pairs (SN910610 and SN011119 followed by R16F2n and R16R2), RFLP, phylogenetic analysis and observation by transmission electron microscope (TEM) were conducted to detect possible $C a$. Phytoplasma. Fifty one samples were positive by nested PCR. Nucleotide sequence showed $99 \%$ similarity to $\mathrm{Ca}$. Phytoplasma accession no. KM280680 from CWB in Viet Nam. Phyologenetic analysis from nucleotide sequence of 16S rDNA classified Thai isolates into 16SrII group. By PCR-RFLP using HaeIII, nucleotides of Thai CWB and Sugarcane white leaf phytoplasma were separated. TEM micrographs of CWB leaves showed phytoplasma like cells with the approximate size of $740-920 \mathrm{~nm}$. The detection of other genes and pathogenicity test will be useful to confirm this possible pathogen.

The value of information across scales for weather-based management decisions

K. A. GARRETT, Plant Pathology Department, University of Florida, Gainesville, FL, USA

Analysis of the value of information offers a useful perspective on the benefits of research, defined in terms of how the additional information will improve decision-making. Uncertainty about weather and climate is a common challenge for decision-making for disease management, across spatial and temporal scales. Decision-making occurs across multiple scales as well, from individual farmers deciding how to manage a disease to global policy makers and donors deciding what technologies to promote and research to fund. Three types of applications for analysis of the value of information will be presented. (1) A common type of decision support in plant disease management is for farmers' tactical decision-making based on recent weather. What approach to additional information about weather-disease relationships is most efficient? How do the cumulative decisions of farmers influence regional disease? (2) As agricultural support programs emphasize insurance programs, there is intriguing potential for financial risk management tools like insurance for plant disease. In this economic framework, what is the value of information for each type of stakeholder? (3) Agricultural development projects with the goal of enhancing crop disease management may benefit from identifying target regions where success is more likely. What is the value of information at different levels of spatial resolution and extent for identifying the best target regions?

Viruses of Ullucus tuberosus: The opportunities and implications of using next generation sequencing in support of statutory diagnostics A. FOX, A. Fowkes, A. Skelton, V. A. Harju, I. Adams, Fera Science Ltd, York, UNITED KINGDOM

Ulluco (Ullucus tuberosus) is a tuber forming crop routinely grown alongside potatoes in the South American Andes, which has become a novel crop in both highland and temperate maritime climates. Eight viruses have been previously reported infecting ulluco, including a strain of Andean potato latent virus (APLV). Ulluco is not currently listed as regulated in EU trade. Following a breach of UK plant health regulations, 400 ulluco plants from a small holding in South-West England were tested for the presence of quarantine viruses using ELISA and real-time PCR. Following positive ELISA results for APLV and multiple other viruses, virus identification was confirmed by Next Generation Sequencing (NGS) using a ribosomal RNA (rRNA) depleted total RNA approach on an Illumina MiSeq platform. Analysis of viral contigs indicated the presence of several novel viruses closely related to, but not consistent with, the viruses indicated by ELISA or those previously described from ulluco. A second outbreak site linked to internet trade in ulluco tubers was investigated using the same approach as described and a similar but distinct suite of viruses was detected. Using NGS in a diagnostic confirmation role offers the ability to identify multiple viruses simultaneously, whilst alleviating the concerns over novel findings without supporting diagnostic context. The limitations of list-based regulatory systems in the era of internet trade will be highlighted.

The Proposal for an International Year of Plant Health in 2020

R. LOPIAN, Ministry of Agriculture and Forestry - Animal and Plant Health Unit - Food Department, Helsinki, FINLAND

The productivity of crops grown for human consumption is increasingly at risk due to the incidence of pests, such as weeds, pathogens and animal pests. In particular pests which have been introduced into new ecosystems can have devastating effects on food security and the environment. The vast increase in the international trade as well as the effects of climate change epitomizes a dramatic increase in the risk of pest introductions. New pathways and disturbed ecological conditions set the perfect conditions for future pest epidemics of major proportions. While the pest risks are increasing globally, resources committed to plant health are reduced, often significantly so, in most countries. Plant health research, taxonomical and diagnostic services are diminishing on national levels. Plant protection services world-wide are facing more and more risks with fewer resources available. It is crucial to strengthen plant health programmes and initiatives to prevent losses to agricultural production and biodiversity. To address these challenges, Finland thought it would be necessary to raise public awareness about plant health and its benefits for the global society by declaring the year 2020 as the International Year of Plant Health (IYPH). An IYPH would serve as a trigger to make national, regional and international plant health systems stronger and more responsive to face the plant health challenges of the future. It will also create a stimulus for better and environmentally sounder agricultural production and for research in plant pathology and entomology. The IYPH will be decided at the next meeting of the UN General Assembly in 
November 2018. Plant pathology professionals at all levels should promote the IYPH with their counterparts in civil society, industry and government and should consider events and actions to be undertaken during the IYPH.

Molecular Mechanism of Strawberry Fusarium Wilt Suppressive Soil

D. R. Kim (1), Y. S. KWAK (2), (1) Gyeongsang National Univ, Jinju-si, KOREA; (2) Gyeongsang National University, Jinju, KOREA

Crops lack genetic resistance to most necrotrophic pathogens. To compensate for this disadvantage, plants recruit antagonistic members of the soil microbiome to defend their roots against pathogens and other pests. The best examples of this microbially based defense of roots are observed in diseasesuppressive soils in which suppressiveness is induced by continuously growing crops that are susceptible to a pathogen, but the molecular basis of most is poorly understood. Here we report the microbial characterization of a Korean soil with specific suppressiveness to Fusarium wilt of strawberry. In this soil, an attack on strawberry roots by Fusarium oxysporum results in a response by microbial defenders, of which members of the Actinobacteria appear to have a key role. We also identify Streptomyces genes responsible for the ribosomal synthesis of a novel heat-stable antifungal thiopeptide antibiotic inhibitory to $F$. oxysporum and the antibiotic's mode of action against fungal cell wall biosynthesis. Both classical- and community-oriented approaches were required to dissect this suppressive soil from the field to the molecular level, and the results highlight the role of natural antibiotics as weapons in the microbial warfare in the rhizosphere that is integral to plant health, vigor and development.

Identification of a torradovirus-encoded protein that complements the systemic movement of a potexvirus lacking the TGB3 gene J. JIMÉNEZ POLO, International Center for Tropical Agriculture (CIAT), Palmira, COLOMBIA

Cassava torrado-like virus (CsTLV, Family Secoviridae) is found in mixed virus infections associated with severe disease symptoms in leaves and roots of cassava (Manihot esculenta Crantz, Euphorbiaceae), which is an important staple food crop for Africa, Asia and Latin America. The virus has been reported in Colombia, Peru and Argentina, where it has a significant effect on the accumulation of total sugars in cassava storage roots. CsTLV encodes an atypical Maf/HAM1 domain not found in other torradovirus genomes, but is present in heterologous viruses of the family Potyviridae also infecting euphorbiaceous hosts. Most viruses encode RNA silencing suppressors (RSS) to counteract the RNA silencing-based plant defense system. However no RSS has been yet identified in torradoviruses. To advance the characterization of CsTLV, agroinfiltration in Nicotiana benthamiana leaves of different viral genes was carried out using GFP as a reporter. Like the Maf/HAM1 domain found in Cassava brown streak virus (CBSV; Genus: Ipomovirus), the one encoded by CsTLV did not show RSS activity. On the other hand, we found that agroinfiltration of RNA2-ORF1 had a significant effect in the establishment of systemic infection by Cassava virus X (CsVX; Genus: Potexvirus), a virus lacking one of the Triple Gene Block genes (TGB3), which is involved in potexvirus movement. Although we observed that single infection of CsTLV increased the expression of GUS in of GUS-transgenic cassava lines, none of the CsTLV genes in this study showed RSS activity.

Rice hormone response is involved in the temperature-dependent function of $\mathrm{Xa}$-mediated bacterial blight resistance S. COHEN (1,2), H. Liu (1,3), V. M. Verdier (1,4), J. E. Leach (5), (1) Department of Bioagricultural Sciences and Pest Management, Colorado State University, Fort Collins, CO, USA; (2) Cell and Molecular Biology Graduate Program, Colorado State University, Fort Collins, CO, USA; (3) Institute of Crop Sciences, Chinese Academy of Agricultural Sciences, Beijing, CHINA; (4) IRD, Cirad, Univ Montpellier, IPME, Montpellier, FRANCE; (5) Colorado State University, Fort Collins, CO, USA

Bacterial blight, caused by Xanthomonas oryzae pv. oryzae (Xoo), causes significant losses to global rice production. Bacterial blight disease is more severe during periods of high temperature. However, little is known about the underlying host molecular responses to pathogen infections during high temperature stress. Most known mechanisms of rice resistance to bacterial blight lose efficacy at high temperature, but, notably, one resistance gene, $\mathrm{Xa}$, functions better. To provide insights into how $\mathrm{Xa} 7$ directs the host response at high temperature, we conducted a transcriptomics experiment. Plants exhibiting resistance at high temperature significantly down-regulated the abscisic acid signaling pathway, which is important to abiotic stress responses, while plants in a susceptible interaction did not. Intriguingly, the salicylic acid response - an important pathway in rice defense to Xoo - was downregulated at high temperatures in all interactions, suggesting that enhanced $\mathrm{Xa} 7$ activity at high temperature is independent of salicylic acid-mediated defense pathways. These results suggest that plants exhibiting $X a 7$-mediated resistance prioritize response to pathogen over response to abiotic stress This ongoing study is providing insight into the trade-off between disease resistance and plant productivity that will help in the development of rice varieties in a changing climate.

Transcriptome sequencing reveals novel Citrus bark cracking viroid (CBCVd) variants from citrus and their molecular characterization Y. WANG (1), C. Zhou (2), M. Cao (3), (1) Citrus Research Institute, Southwest University, Chongqing, CHINA; (2) Southwest University, Chongqing, CHINA; (3) Southwest University, Chongqiang, CHINA

Citrus bark cracking viroid (CBCVd) has been identified as an important causative agent on citrus and hop. In this study, we obtained the full-length genomes of different variants of citrus viroids from Pakistan through transcriptome sequencing. CBCVd variants were first found in Pakistan. These newly discovered Pakistani CBCVd variants were provisionally named as 'CBCVd-LSS' for their low sequence similarity (80.9\%-88.9\%) with the CBCVd RefSeq sequence (NC_003539). Two most predominant CBCVd sequences from Pakistan had the highest, $91 \%$ and $88 \%$ identity with two CBCVd sequences isolated from hop, respectively. Identification and molecular characterization of CBCVd from citrus in Pakistan and China were also reported. The length of CBCVd from China ranged from 282 to 286 nucleotides, while from Pakistan it ranged from 273 to 277 nucleotides. Based on genetic diversity and phylogenetic analysis, two main CBCVd clades were identified. CBCVd sequences were further divided into six sub-clades. Sequence alignment revealed some nucleotide changes between these sub-clades, and analysis indicated that several mutations could significantly affect the primary and secondary structure of the viroid. Our results indicated that the CBCVd sequences from Pakistan and China were significantly different and Pakistan might be one of the independent geographical origins of CBCVd worldwide.

Global wheat stem rust monitoring: Meeting the challenges of a re-emerging threat to wheat production D. HODSON (1), Y. Jin (2), P. D. Olivera Firpo (3), L. J. Szabo (4), M. S. Hovmøller (5), M. Patpour (5), K. Nazari (6), C. A. Gilligan (7), M. Meyer (7), M. C. Hort (8), S. Millington (9), Z. A. Pretorius (10), R. F. Park (11), T. G. Fetch Jr. (12), J. Grønbech Hansen (13), (1) CIMMYT, Addis Ababa, ETHIOPIA; (2) USDA-ARS Cereal Disease Laboratory, Saint Paul, MN, USA; (3) University of Minnesota, St. Paul, MN, USA; (4) USDA ARS, St Paul, MN, USA; (5) Aarhus University, Slagelse, DENMARK; (6) ICARDA, Izmir, TURKEY; (7) University of Cambridge, Cambridge, UNITED KINGDOM; (8) UK Met Office, Exeter, UNITED KINGDOM; (9) Uk Met Office, Exeter, UNITED KINGDOM; (10) University of the Free State, Bloemfontein, SOUTH AFRICA; (11) Univ of Sydney Plant Breeding Institute, Cobbitty, AUSTRALIA; (12) Agriculture and Agri-Food Canada, Brandon, MB, CANADA; (13) Aarhus University, Aarhus, DENMARK 
Wheat stem rust, one of the most destructive disease on one of the world's primary staple food crops is considered by some to be a conquered past threat. Or is it? Epidemics caused by highly virulent races in East Africa in the last two decades raised concerns amongst global wheat scientists that stem rust had returned as a significant threat to wheat production after decades in abeyance. As a result, a global rust monitoring system focussed on stem rust was initiated. The characteristics, evolution and successes of this global monitoring system are described. Over the last decade, an international consortium has developed one of the most comprehensive and wide reaching crop disease monitoring systems, covering nearly 40 countries. Extensive surveillance networks and diagnostic platforms are now supported by state of the art data management systems and advanced modelling / forecasting tools. The current status, characteristics and threats posed by new stem rust race groups in East Africa, Central Asia, Russia and Europe are highlighted. Increasing vulnerability of North American wheat production to exotic race incursions is also considered. Whilst monitoring has been successful, the case is presented that wheat stem rust is re-emerging rapidly as a significant threat to global wheat production that needs increased and intensified collaborative research effort and attention.

Investigation of leaf blotch and blight disease pathogens of Microstegium vimineum and screening for its potential biological control agents S. QIANG (1), R. Ding (1), Q. Huang (1), L. Zhang (1), Y. Wu (2), R. C. Reardon (3), (1) Weed Research Laboratory/Nanjing Agricultural University, Nanjing, CHINA; (2) USDA Forest Service, Morgantown, WV, USA; (3) USDA Forest Service-Forest Health Assessment \& Applied Science Team, Morgantown, WV, USA

Microstegium vimineum (Trin.) A., native to East and Southeast Asia, is an annual gramineous weed that has invaded the eastern United States of America and rapidly become a vicious invasive weed in the country. We evaluated a large number of microorganisms associated with leaf-blotch and leaf-blight diseases (434 leaf samples) on the weed in 11 Chinese provinces from 2014 to 2016 for potential biocontrol agents. The assessment was based on Koch's postulate, morphological and molecular identification of the organisms. A total of 381 isolates, including 82 pathogenic fungal isolates, were obtained. These fungal pathogens belonged to 19 species of 10 common genera, including Alternaria, Bipolaris, Corynespora, Curvularia, Drechslera Exserohilum, Gloeocercospora Nigrospora, Pestalotiopsis and Phoma. Among them, five species that caused apparent disease on M. vimineum had not been reported on the host previously. Furthermore, eight isolates with strong virulence to this weed were assessed for biocontrol efficacy and host specificity in both controlled- environment and field trials; the candidates B. panici-miliacei, B. maydis and Curvularia geniculata demonstrated the greatest potential as biological control agents against $M$. vimineum and other grassy weeds.

Phytophthora betacei and Phytophthora andina: Controversy within the Clade 1c? M. Mideros (1), M. Parra (2), N. Guayazan (1), G. Danies (1), S. RESTREPO (1), (1) Universidad de los Andes, Bogota, COLOMBIA; (2) Universidad de Los Andes, Bogot, COLOMBIA

In South America, late blight is one of the main threats to several domesticated and semi-domesticated crops. A recently described species, Phytophthora betacei, has been found causing symptoms akin to late blight on tree tomato crops (Solanum betaceum) in Colombia. The description of this new species led us to redefine the previously described polyphyletic species $P$. andina. This species has been controversial since its designation It has been hypothesized to have arisen from hybridization based on the conflicting phylogenetic information of mitochondrial and nuclear genealogies. Other researchers have considered that the polyphyletic nature of the mitochondrial alleles renders $P$. andina an invalid species. The identification of $P$. betace as a different species from $P$. infestans sheds some light on the origin of $P$. andina. In this study we describe the biology, the life cycle, the disease cycle, the sexual incompatibilities, the distribution and several physiological responses, including fungicides' responses, of the new species, $P$. betacei. All results suggest that indeed, $P$. betacei is a separate species, where pre- and postzygotic mechanisms played a role in the speciation process. We also explored the genome of $P$. betacei and discussed the possible causes of its expansion and the mechanisms used to infect its only described host, Solanum betaceum.

\section{Emerging diseases in horticultural crops}

M. L. GULLINO (1), J. Thomas (2), G. Gilardi (1), A. Garibaldi (1), T. Wood (2), R. Caiazzo (2), (1) Agroinnova - University of Torino, Grugliasco, Torino, ITALY; (2) National Institute of Agricultural Botany, Cambridge, UNITED KINGDOM

During recent years, many new diseases caused by foliar and soilborne pathogens have appeared for the first time worldwide on a number of vegetable crops and some diseases re-emerged as a consequence of globalization and climate change. This review will cover the emergence of new diseases in the vegetable sector and few re-emerged ones such as: Peronospora belbahrii on basil; Fusarium equiseti on lettuce, rocket and radish; Myrothecium verrucaria and M. roridum on lamb's lettuce, spinach and wild rocket; Allophoma tropica on lettuce, Phytophthora capsici and Fusarium solani f. sp. cucurbitae on zucchini, F. oxysporum f. sp. cucumerinum on cucumber and P. nicotianae on tomato. New species of Pythium (P. aphanidermatum, P. irregulare, $P$. dissotocum, $P$. sylvaticum) have frequently been isolated on spinach and swiss chard, lamb's lettuce, lettuce and bean. Many of the pathogens described are seed transmitted, a biological characteristic that raises the risk of disseminating diseases worldwide.

Manipulation of the soil microbial community to suppress soil-borne diseases of banana through soil management

T. PATTISON (1), A. McBeath (2), D. East (2), H. Birt (3), P. Dennis (3), (1) Department of Agriculture \& Fisheries, South Johntone, AUSTRALIA; (2) Department of Agriculture \& Fisheries, South Johnstone, AUSTRALIA; (3) The University of Queensland, St Lucia, AUSTRALIA

Root pathogens, such as plant-parasitic nematodes and Fusarium wilt (Fusarium oxysporum f. sp. cubense) represent key constraints to global banana production. Consequently, farm management strategies are being developed to encourage the growth of organisms that suppress pathogens, while maintaining productivity. A holistic view of soil functions with an understanding of the ecological interactions is required to develop productive, disease suppressive farming systems. Soil nitrogen and organic matter are two factors that have a large impact on soil organisms and can be manipulated by farm management. Their impacts on biological processes were assessed in field trials in the Wet Tropics of Queensland, Australia, by monitoring soil enzyme activities, the induced respiratory responses of soil organisms to multiple substrates (MicroResp ${ }^{\mathrm{TM}}$ ) and by characterising soil nematode, archaeal and bacterial diversity using microscopy and high-throughput phylogenetic marker gene sequencing, respectively. Successional trends indicating a more diverse soil community were observed in all soil biological soil assessments. The OTU-level microbial community composition differed significantly $(\mathrm{p}<0.05)$ between groundcover and nitrogen treatments. Partial RDA was used to visualise differences in the composition of microbial communities, which separated the community associated with bare and vegetated soil and the nitrogen treatments. There was a significant $(p<0.05)$ increase in $\beta$ glucosidase activity under vegetated groundcovers, which was strongly correlated with suppression of Fusarium wilt symptoms determined using a soil bioassays. Our results indicate that manipulation of nitrogen and groundcovers made it possible to manipulate to soil microbiome to contribute to the suppression Fusarium wilt of bananas. 
Resistance breaking tospoviruses in Europe: What is the current situation?

M. TURINA (1), M. Ciuffo (2), (1) Institute for Sustainable Plant Protection, National Research Council, Italy, Turin, ITALY; (2) Institute for Sustainable Plant Protection CNR, Torino, ITALY

Tospoviruses are now included in the order Bunyavirales, family Tospoviridae, genus Orthotospovirus. Currently four tospovirus species are reported in Europe: Tomato spotted wilt orthotospovvirus (TSWV), Impatiens necrotic spot orthotospovirus, (INSV), Iris yellow spot orthotospovirus, (IYSV) and Polygonum ringspot orthotospovirus (PolRSV). Only viruses of the first three species cause diseases in vegetable crops, often being a limiting factor in crop production. Viruses of the first two species are efficiently transmitted by Frankliniella occidentalis and TSWV is by far the most damaging tospovirus causing important diseases in pepper, tomato, artichoke and lettuce crops worldwide. By contrast, IYSV is transmitted by Thrips tabaci, mostly in onion, garlic and leek crops in many countries in Europe and worldwide. Resistance genes for TSWV are reported for pepper, tomato and lettuce, but only those for pepper and tomato have been extensively deployed in Europe. Molecularly, both the resistance genes and their corresponding virus encoded avirulence factors have been characterized in detail. We are reporting on the occurrence of resistance breaking strains in open fields throughout southern and central Europe, for both pepper and tomato crops. Furthermore we will report on the efforts to obtain new resistance for breeding purposes through traditional approaches, in particular for pepper and tomato.

Automated plant disease diagnosis using innovative android App (Plantix) for farmers in Indian state of Andhra Pradesh S. RUPAVATHARAM (1), A. Kennepohl (2), B. Kummer (2), V. Parimi (1), (1) International Crop Research Institute for Semi-Arid Tropics, Hyderabad, INDIA; (2) PEAT GmbH, Berlin, GERMANY

Agricultural extension systems are stretched beyond physical limits while assisting farmers during crop production in the developing world. Information and Communication Technologies are booming worldwide with the introduction of mobile applications in smart phones. Progressive Environment Agriculture Technologies (PEAT), a German startup company developed a mobile application 'Plantix' that assists in detecting damage on plants with the help of a smart phone image. Mobile internet connectivity enables uploading of image (time and geo-tagged) to a cloud based server where machine learning based algorithms are used to process the optical patterns and detect the diseases. This information is conveyed back to the user within few seconds along with suggested knowledge on disease management including biological and chemical control methods. Plantix is presently downloaded by more than 0.520 million users and has an image data base of over 2 million plant damages. Plantix is trained on more than 250 plant damages including diseases, pests and nutrient disorders. Accuracy in identification relies on building a robust data base for every disease through image tagging performed by subject matter experts. For over 100 classes the detection accuracy of plantix is over $90 \%$, however in some cases like early and late leaf spots of peanut an accuracy of over $95 \%$ (15,000 images) is witnessed. It has been observed that accuracy of detection depends upon quality of data set that increases with rise in number of independent images. There is potential in enhancing detection accuracy and include more plant damages and then the challenge to decipher disease complexes.

Survey for cassava brown streak disease revealed for the first time the presence of a molecular variant of Uganda cassava brown streak virus in Zambia

R. M. MULENGA (1), L. Boykin (2), O. Alabi (3), (1) Zambia Agriculture Research Institute, Lusaka, ZAMBIA; (2) The University of Western Australia, Perth, AUSTRALIA; (3) Texas AgriLife Research and Extension Center, Weslaco, TX, USA

A diagnostic survey was conducted in July 2017 in Luapula and Northern provinces of Zambia to investigate the presence or absence of cassava brown streak disease (CBSD) and its associated viruses. A total of 29 cassava fields were visited during the survey and 92 symptomatic and 24 nonsymptomatic cassava leaf samples were collected from 116 plants. Mean CBSD incidence varied across fields but averaged $32.3 \%$ while mean disease severity was 2.3 on a $1-5$ rating scale. RT-PCR screening of all 116 samples with one generic and two species-specific primer pairs yielded DNA bands of the expected size with the generic and Uganda cassava brown streak virus (UCBSV)-specific primers in all symptomatic plants. All 24 nonsymptomatic samples were negative for the virus and all samples tested negative with primers targeting Cassava brown streak virus (CBSV). The complete genome of a representative isolate of UCBSV (WP282) was determined to be 9,050 nucleotide (nt) in length, minus the polyA tail. A comparative analysis of this isolate with global virus isolates revealed its nature as a molecular variant of UCBSV sharing 94/96\% maximum complete polyprotein nt/amino acid identities with isolates from Malawi (MF379362) and Tanzania (FJ039520). This is the first report of CBSD and UCBSV in Zambia thus expanding the geographical distribution of the disease and its associated virus and further reinforcing the need to strengthen 31 national and regional phytosanitary programs in Africa.

Seeing the light: The roles of red and blue light sensing in bacterial plant pathogens G. A. BEATTIE, Iowa State University, Ames, IA, USA

Plants collect, concentrate and conduct light throughout their tissues, thus enhancing light availability to their resident microbes. These microbes have evolved mechanisms of photosensing to modify their behavior in response to light cues. Many plant microbes have both far-red/red- and blue lightsensing proteins, and while the biochemical properties of these proteins are shared among organisms, we are only beginning to understand their biological roles in microbial interactions with plants. Unlike red and blue light, far-red light escapes absorption by photosynthetic tissues and is available for extensive redistribution through plants. Recent studies with foliar bacterial pathogens demonstrate that far-red light functions as a potent signal that influences global gene expression. Moreover, far-red light-sensing proteins contribute to suppressing virulence and behaviors such as swarming motility that influence the virulence of these pathogens. Blue light-sensing proteins also contribute to suppressing bacterial virulence, suppressing plant defenses, and increasing pathogen adherence to leaves and preventing entry, as well as to enhancing symbioses by a nitrogen-fixing symbiont. An increased understanding of how photosensing contributes to the evasion of light-mediated plant defenses and integrates with pathogen responses to co-occurring environmental stresses is increasing our appreciation of the biological significance of photosensing in plant microbe lifecycles.

Relationship between soil health indicators and potato early-die in Michigan

G. W. BIRD, Michigan State University, East Lansing, MI, USA

Potato early-die (PED) caused by the joint action of Pratylenchus penetrans and Verticillium dahliae is a limiting factor in potato production in Michigan. The objective of this research was to identify the relationships between soil health indicators and PED. Two 50-acre potato fields were used. The Defender was a conventional two-year rotation with potato $(2015,2017)$ and seed corn $(2014,2016$, with soil fumigation and a biological nematicide. The Challenger had one cash crop (potato in 2015, 2017) and cover crops $(2014,2016)$, pearl millet (2014) and cover crop blends (2016). Annual soil samples from 32 permanent geo-positioned sites were analyzed for twelve soil health indicators, nematodes, bacteria, tuber yields and aerial NDVI (vegetative index). Mean Defender tuber yields were $371 \mathrm{cwt} / \mathrm{A}$ in 2015; whereas, Challenger yields were $293 \mathrm{cwt} / \mathrm{A} .2016$ soil health and NDV 
indices were greater for the Challenger than the Defender. The 2016 NDVI was superior for a four cultivar cover crop blend, compared to a seven species blend. The 2016, bacteria DNA sequences identified 28 phyla, 81 classes, 140 orders, 300 families and 814 genera. Sequence assignment was dominated by 9 of the 28 phyla recovered, comprising nearly $96 \%$ of the total bacterial community assemblage and present in all GPS locations. Every sample contained known beneficial bacterial taxa including Proteobacteria, Acidobacteria, Actinobacteria, Verrucomicrobia, Firmicutes. The greatest 2017 tuber yields were associated with the 2016 four cultivar cover crop blend and the aerial NDVI. The highest 2017 P. penetrans populations were associated with the greatest tuber yields.

Sentinel arboreta as 'bridge environment' to study novel host-pathogens interactions and detect potentially alien plant pathogens C. MORALES-RODRIGUEZ (1), T. Dogmus-Lehtijarvi (2), S. Woodward (3), A. G. Aday Kaya (2), F. Oskay (4), A. Vannini (1), (1) DIBAFUniversity of Tuscia, Viterbo, ITALY; (2) Suleyman Demirel Univ, Isparta, TURKEY; (3) Univ of Aberdeen, Aberdeen, SCOTLAND; (4) Çankırı Karatekin University, Ankara, TURKEY

The sentinel arboreta concept has been recently proposed as an efficient strategy to identify potential exotic plant pathogens aggressive to native tree species from a geographical area. In fact, the close contact in the same arboretum of tree species from different areas in the worlds facilitates host jump by potential plant pathogens and expression of novel host-pathogen interactions. In fall 2015 a survey was carried out on about 40 different oak species from Europe, Asia and America planted together in a section of the Ataturk arboretum in Istanbul (Turkey). An array of foliar and bark symptoms were recorded on inspected oak species. Some foliar symptoms were shared among species from different geographical areas. Several fungal morphotypes were consistently associated with specific symptoms. Morphological and molecular detection were attempted to each fungal endophytes. Symptomatic and no-symptomatic tissues were processed by Illumina platform. The results indicate the efficiency of this approach to highlight the risk of host jump and insurgence of novel host-pathogen interactions between reciprocally alien species.

Maintaining diverse culture collections ensures adequate resources and capability to support plant biosecurity and global trade N. SPENCE, Department for Environment, Food \& Rural Affairs (United Kingdom), York, UNITED KINGDOM

The long-term trend of increasing volume and speed of movement of plants and other material traded from an increasing variety of sources increases the chances of exotic pests arriving with imported goods and travellers, as well as by natural means. The GB plant biosecurity strategy ensures activity is directed at priority pests and pathways and is informed by comprehensive risk assessment, which includes plant pathology, population dynamics, and epidemiology, as well as social sciences to understand the values at stake. To respond effectively to new and emerging threats, GB as a whole must be resilient, capable and prepared to respond flexibly to new and emerging threats to ensure GB production has a good reputation to allow exports of plants and plant products to develop, with consequent economic and social benefits. Plant pest culture and reference collections are fundamental for supporting accurate diagnosis of plant pests. The capability of National Plant Protection Organisations to reliably identify these organisms is critical for effective phytosanitary measures, to ensure evidence-based policy on new and emerging plant pests and to facilitate the validation of new molecular protocols. In England there are several important culture collections of viruses and phytoplasmas, fungi, bacteria, nematodes and insects which are maintained and curated to support plant biosecurity which will be presented and their application discussed.

Evaluation of weather-based foliar fungicide applications for soybean in the mid-Atlantic U.S.

T. ZHOU (1), D. L. Holshouser (1), H. L. Mehl (2), (1) Virginia Tech, Suffolk, VA, USA; (2) Virginia Tech Tidewater AREC, Suffolk, VA, USA

Foliar fungicide applications are a standard practice for some growers, however, fungicides increase soybean yield less than one third of the time in the mid-Atlantic U.S. Yield response to fungicides depends, in part, on the presence of a pathogen and conducive environmental conditions for disease development. The objective of this study was to compare crop developmental stage-based fungicide applications to weather-based applications for foliar disease control and yield response in soybean. On-farm replicated strip trials were conducted at 21 locations from 2014 to 2016. Treatments included no fungicide, fungicide applied at beginning pod (R3), and fungicide applied when environmental conditions favored disease $\left(18-26^{\circ} \mathrm{C}\right.$ and $\geq 10$ hours relative humidity $\geq 95 \%$ for 2 consecutive days). Foliar disease was rated bi-weekly and yield was measured at harvest. Frogeye leaf spot and Cercospora leaf blight were the major diseases observed. Environmental parameters triggered fungicide applications at 20 of the 21 locations, but yield response was observed at only 7 locations. Weather-based fungicide applications did not differ in yield compared to R3 applications. However, when environmental parameters conducive for disease occurred within 2 weeks of the R3 stage, R3 applications were more likely to increase yield. Results indicate both crop developmental stage and environmental conditions should be considered prior to applying foliar fungicides in mid-Atlantic soybean.

Advanced Precision Spray Application Technology for Effective Control of Ornamental Diseases

H. ZHU, PH.D. (1), A. Fulcher (2), R. L. Rosetta (3), M. W. Wallhead (1), (1) USDA-ARS, Wooster, OH, USA; (2) University of Tennessee, Knoxville, TN, USA; (3) Oregon State Univ, Aurora, OR, USA

Advancing conventional sprayer technology with precision variable-rate capability is an economically feasible method to solve current challenges associated with imprecise application and extensive waste of foliar-applied pesticides and other products. An experimental laser-guided precision sprayer was developed to adjust spray outputs based on crop canopy architectures in real time. The sprayer could apply different rates to different sections of individual plants. On-farm field tests in commercial nurseries in Ohio, Oregon and Tennessee demonstrated the insect and disease control efficacies of this experimental sprayer were comparable to those of conventional sprayers, while the new sprayer reduced average pesticide use by $46 \%$ to $70 \%$, airborne spray drift by up to $87 \%$ and spray loss on the ground by $70 \%$ to $90 \%$, with annual chemical savings by $\$ 140$ to $\$ 281$. Based on the success of this sprayer development, a versatile laser-guided spray control system was developed as a retrofit for air-blast sprayers used in ornamental nurseries and boom sprayers in greenhouses. This new variable-rate spray system will significantly advance conventional spray technologies and offer an environmentally responsible and sustainable approach to controlling insects and diseases and applying other foliar products in future nursery and greenhouse production.

Importation of Plant-associated Microbial Cultures: What Are the U.S. Regulatory Requirements? Z. LIU, USDA APHIS PPQ, Riverdale, MD, USA

One of the primary missions of The U.S. Department of Agriculture, Animal and Plant Health Inspection Service, Plant Protection and Quarantine (USDA-APHIS-PPQ) is to safeguard America's agricultural and natural resources from the introduction and spread of exotic plant pests of economic and environmental significance. PPQ regulates live plant pests and biological control organisms. PPQ-PPBP issues thousands of permits and associated documents each year for the importation, interstate movement, and continued curation of these organisms. Many of these permits are intended for diagnostic, laboratory, growth chamber, greenhouse, and/or field research. PPQ permits are generally required for the importation of plant-associated 
microbes into the United States and its territories. Foreign isolates are considered exotic, even when the same species is common in the U.S. We require that research with these organisms be performed in a USDA APHIS-inspected containment facility. A PPQ permit may not be required for the importation of pure cultures of microbials that are not plant pathogens or do not possess biopesticidal properties since this falls outside of our jurisdiction. However, other permits may be required from agencies such as APHIS Veterinary Services, APHIS Biotechnology Regulatory Services, Centers for Disease Control, Environmental Protection Agency and others. To facilitate the global research using microbes to increase agricultural productivity, PPQ strives to ensure that permitting decisions are as timely, consistent, risk-based and transparent as possible.

\section{Late blight pathogen diversity in North-Eastern Europe}

R. KIIKER (1), D. Cooke (2), I. Skrabule (3), A. Ronis (4), E. Runno-Paurson (1), (1) Estonian University of Life Sciences, Tartu, ESTONIA; (2) James Hutton Institute, Dundee, SCOTLAND; (3) Institute of Agricultural Resources and Economics, Priekuli Research Centre, Priekuli, LATVIA; (4) Institute of Agriculture, Lithuanian Research Centre for Agriculture and Forestry, Akademija, LITHUANIA

Crop loss and economic costs can be substantial, when conditions for late blight (Phytophthora infestans) development and spread are favourable in the Baltic countries (Estonia, Latvia and Lithuania). For effective late blight management, knowledge of the contemporary pathogen population in the region is valuable. To reveal $P$. infestans genotypes and genetic diversity in the Baltic countries over a three-year-period, a 12-plex SSR marker assay was applied to a collection of $P$. infestans isolates from potato. Overall genetic diversity in the pathogen population was highest in Estonia, followed by Latvia and Lithuania. Most of the multilocus genotypes (MLGs) were identified from a single field outbreak and no MLGs were common to three countries. In Lithuania, the most common local clone was found 19 times over two sampling years whereas, in Latvia, another local clone was found 10 times in one year. In those cases, the samples were from a local cluster of outbreaks. Other repeatedly found local clones were mostly sampled in one year of the study from a single field outbreak and represented by 2-6 isolates. However, none of the clonal lineages found in Western Europe was detected from the Baltic countries. This study reveals the high genetic diversity and possible sexual reproduction in $P$. infestans populations from the Baltic countries. Most of the P. infestans isolates have unique MLGs with unpredictable phenotypic traits, which may complicate disease management.

Plant pathology in China enters into a new era

Y. L. PENG (1), C. Han (1), W. Sun (2), (1) China Agricultural University, Beijing, CHINA; (2) Department of Plant Pathology, China Agricultural University, Beijing, CHINA

The Chinese Society for Plant Pathology (CSPP) was established in 1929. With nine decades of development, CSPP has become one of the largest academic societies in plant pathology in the world, consisting of 16 subject committees with more than 6500 members. Since 2004, the society has organized and sponsored annual meetings, which have attracted more than a thousand participants every year, and provided an important platform for Chinese plant pathologists to exchange their research achievements and for students to communicate with established scientists. For the past five years, the Chinese central government has invested more than two billion RMB in the investigation of important crop diseases. In addition, the National Natural Science Foundation of China funded numerous plant disease-related projects in the recent years. These inputs have allowed Chinese plant pathologists to achieve significant progress in diverse topics of plant pathology. CSPP has been a member of the International Society of Plant Pathology since 1983, and is a founding member of the Asian Association of Societies for Plant Pathology, which was established in 2000. CSPP has also had close collaboration with the American Phytopathological Society since 2007. The active involvement of Chinese plant pathologists' in domestic plant disease management, along with participation in international initiatives, has enabled plant pathology in China to enter into a new era.

\section{Fusarium toxisomes may be necessary for synthesis of high levels of deoxynivalenol and production of the distinct sesquiterpene mycotoxin,} culmorin

C. Flynn (1), G. Tang (2), M. Boenisch (1), A. Blum (3), K. Broz (4), Y. Chen* (2), C. Schmidt-Dannert (1), Z. Ma (2), C. KISTLER (4), (1) University of Minnesota, Saint Paul, MN, USA; (2) Institute of Biotechnology, Zhejiang University, Hangzhou, CHINA; (3) University of Queensland, Brisbane, AUSTRALIA; (4) USDA ARS, Saint Paul, MN, USA

The fungus Fusarium graminearum causes the disease Fusarium head blight on wheat and contaminates grain with sesquiterpene mycotoxins such as deoxynivalenol (DON) and culmorin. Induction of mycotoxin synthesis is associated with profound subcellular differentiation with enzymes of the DON biosynthetic pathway localizing to highly modified endoplasmic reticulum (ER) structures organized as stacks of smooth ER cisternae. These structures have been called toxisomes. Super-resolution microscopy suggests that trichodiene synthase (Tri5), a cytosolic enzyme catalyzing the first step in DON synthesis, is concentrated in regions within and around toxisomes. Several lines of evidence indicate that toxisome structure is not only required for high level DON synthesis but also for production of the sesquiterpene culmorin, that is synthesized by a separate pathway. Deletion mutants ( $\Delta$ tri5) fail to form toxisomes and lack the ability to make culmorin. Replacing Tri5 with an enzymatically inactive, but structurally similar tri5 $5^{\mathrm{N} 225 \mathrm{D} / \mathrm{S} 229 \mathrm{~T}}$ allele restores both toxisome structure and culmorin synthesis. Treatments that prevent F-actin assembly (latrunculin b) or that target the myosin I motor (phenamacril) prevent toxisome formation and greatly reduce trichothecene synthesis suggesting a role for the actin cytoskeleton in toxisome formation. These results suggest that the toxisomes of $F$. graminearum are necessary for high level sesquiterpene synthesis.

Xylella fastidiosa - Insect Vector Interactions: Current and Potential Future Research Directions

J. LOPES, Departamento Entomologia e Acarologia, Universidade de São Paulo, Piracicaba, BRAZIL

Vectors play a major role in epidemiology of diseases caused by Xylella fastidiosa. Information on vector ecology, behavior and transmission sets the basis for identifying key species involved in pathogen spread and approaches to manage the diseases. The bacterium is non-circulative (foregut-borne), lacking latent period and transstadial transmission, but it is persistently-transmitted after acquisition by adults due to biofilm formation and propagation on the vector retention sites (e.g. the cuticular lining of the precibarium). The ability to sequence and transform X. fastidiosa, combined with advanced studies employing functional genomics, vector acquisition of cultured cells from artificial diets, microscopy and PCR-based assays, allowed the development of a model for bacterial adhesion and biofilm formation in the vector's foregut. Host selection, electrical penetration graph (EPG) and acoustic communication studies have indicated vector behavioral aspects that could be manipulated to manage vector population and/or reduce pathogen transmission. Transmission efficiency is markedly influenced by $X$. fastidiosa populations in source plants (and maybe in the vectors), suggesting that approaches targeting bacterial growth may be useful to reduce transmission rates. Future research should translate fundamental knowledge of $X$. fastidiosa-vector-plant interactions into new ways to disrupt the transmission process and generate sustainable management strategies.

Impact of climate change on fungal disease of crops commonly grown in Bangladesh

M. M. ISLAM (1), L. Rahman (2), B. Meah (3), B. Goswami (2), (1) Bangladesh Agricultural Research Institute, Joydebpur, BANGLADESH; (2) Bangladesh Agricultural Research Institute, Gazipur, BANGLADESH; (3) Bangladesh Agricultural University, Mymensingh, BANGLADESH 
Crop diseases are remarkable constraints to the production of food for the rapidly expanding population of Bangladesh. Exact crop losses due to diseases yet to estimate in the country. However, world reports reveal that plant disease causes 9 to $16 \%$ losses in widely grown food crops. Duration and time of warm weather and rainy season as well as annual number of rainy days and amount of rainfall are being changed remarkably. Reports from other countries clearly show that incidence and severity of plant diseases are directly influenced by the climate changes. Climate change has emerged as the greatest threat facing humankind today. In Bangladesh and some other countries, prolonged drought has significantly affected production of summer crops. Average temperature of Bangladesh varies from 25.62 to 26.14 and relative humidity varies from 79.81 to $80.90 \%$ during the period 1950 to 2010 . Irregular and fluctuating trend of rainfall is observing in the last 6 decades. Extension of soil salinity is remarkable in the country. Now the Salt affected area of Bangladesh is 30\%, whereas it was 3.5\% in 1973. Ground water level is falling 35.4 feet to 53.8 feet from 2005 to 2009 . Analyzing current trends and scenarios based on projected temperature increases, the study warns that Bangladesh is vulnerable yield loss crops due to incidence of pests and diseases. Total number of crop diseases was 316 in 1980's, 586 in 1990's, 795 in 2000's and 1539 in 2010's.

Grapevine trunk diseases: A complex of related pathogens with global impacts M. R. Sosnowski (1,2), D. Gramaje Perez (3), J. R. Urbez-Torres (4), L. MUGNAI (5), (1) South Australian Research and Development Institute (SARDI), Urrbrae, AUSTRALIA; (2) University of Adelaide, Adelaide, AUSTRALIA; (3) Instituto de Ciencias de la Vid y el Vino (ICVV), Logroño, SPAIN; (4) Agriculture and Agri-Food Canada - Summerland Research and Development Centre, Summerland, BC, CANADA; (5) DISPAA, University of Florence, Firenze, ITALY

Grapevine trunk diseases (GTDs) have become a major concern worldwide, causing significant economic impact by reduced production and vineyard longevity. Petri disease and black foot affect young grapevines whilst the diseases Eutypa, Botryosphaeria and Phomopsis dieback, and esca affect mature grapevines. These diseases are caused by a wide range of fungal pathogens producing a range of symptoms including: leaf and shoot distortion and discolouration, external wood cankers and dieback, internal wood necrosis and staining, poor growth, mortality of roots and sudden vine collapse. The prevalence of GTDs has significantly increased with changes in production practices, loss of effective chemicals, predominance of susceptible cultivars and ageing of vineyards. Pruning wounds are the main infection portal for these pathogens and inoculum sources include a wide range of alternative hosts such as fruit crops and many introduced and/or native tree species. Due to the rapid expansion of wine regions, young vine disease is escalating, as pathogens can be introduced during propagation, and diseases are often associated with poor planting practices and stress. Control is limited to hot water treatment and fungicide dips, with mixed results. With the rapid development of molecular diagnostic capabilities, it is important to understand infection thresholds and the link with disease in the vineyard. A good understanding of the etiology, biology and epidemiology of GTD fungi has led to the development of effective management strategies in the vineyard, through wound protection and remedial surgery. Successful disease management requires a holistic approach from the nursery to the vineyard.

Developmental dynamics of the Northern root-knot nematode (Meloidogyne hapla) in Washington State vineyards K. EAST (1), I. A. Zasada (2), R. P. Schreiner (2), M. M. Moyer (1), (1) Washington State University, Prosser, WA, USA; (2) USDA ARS, Corvallis, OR, USA

The Northern root-knot nematode, Meloidogyne hapla, is an economically-important soilborne pest in Washington State wine grape vineyards. There is limited information available on the biology of this nematode upon which to base management decisions, such as appropriate timing for chemical management tactics. The objective of this study was to determine when vulnerable life stages of $M$. hapla are present in Washington vineyards to improve the timing of management intervention. Prediction of when these time periods occur can be accomplished based on heat accumulation in soil. Two different commercial vineyards with M. hapla were sampled from 2015 to 2017 (March to March). Soil samples were collected weekly during the growing season and monthly from October to March to determine densities of infective second-stage juveniles (J2), eggs of M. hapla, and fine root tips of grapevine. Life stages were considered within the context of soil growing degree days calculated from a base temperature of $0^{\circ} \mathrm{C}\left(\mathrm{GDD}_{\text {soil }}\right)$ starting in March. Meloidogyne hapla has a single generation per year, with J2 density peaking over the winter (4291-4881 GDD soil ), then declining to a low density in late June to early July (1895-2379 GDD soil $)$. Egg density peaked in late July to August (2871-3069 $\left.\mathrm{GDD}_{\text {soil }}\right)$. Similar to the egg peak, fine root tip density peaked at 2379-2871 GDD soil. A model for prediction of M. hapla J2 development in Washington wine grape vineyards based on GDD soil $_{\text {will be }}$ presented.

Forging new tools for the war against Bactericera cockerelli and "Candidatus Liberibacter solanacearum": A pathosystem on the move I. E. BADILLO-VARGAS, Texas A\&M AgriLife Research, Weslaco, TX, USA

The potato psyllid, Bactericera cockerelli, is the insect vector of the fastidious bacterium "Candidatus Liberibacter solanacearum" (Lso). This bacterial pathogen causes diseases in several solanaceous crops, including zebra chip, an economically important disease of potatoes in United States, Mexico and New Zealand. Lso is transmitted in a persistent propagative manner by B. cockerelli where it infects and multiplies in the digestive track, reproductive organs, and salivary glands of its insect vector. The persistent nature of this insect-bacterium interaction complicates matters even more as the vector can potentially be a reservoir of bacterial inoculum in the absence of its cultivated host plants. Currently, vector and pathogen control relies extensively on the use of a limited number of insecticides. This strategy is neither effective or sustainable as recent studies have shown that insecticide resistance development to neonicotinoids by $B$. cockerelli has already occurred in Texas. Therefore, alternative strategies of control are needed to effectively minimize losses caused by this vector-borne bacterial pathogen. This presentation will feature the evaluation of diverse management strategies with the final goal of developing an integrated pest management program for vegetable production that might have worldwide implications as this pathosystem continues to move across borders into new geographic areas.

Function of pathogenesis-related protein 10 (PR10) in soybean resistance to Asian soybean rust (ASR)

D. HU (1), S. Park PhD (2,3), M. Ganiger (3), C. Zhang (4), Z. Y. Chen (1), (1) Louisiana State University Agricultural Center, Baton Rouge, LA, USA; (2) LSU Agcenter, Baton Rouge, LA, USA; (3) LSU Agcenter, Baton Rouge, LA, USA; (4) Alcorn State University, Alcorn State, MS, USA

ASR, which is caused by Phakopsora pachyrhizi, is one of the most important soybean diseases worldwide. Currently, this disease is managed through fungicide application due to the fact that all commercial soybean cultivars are susceptible to P. pachyrhizi. In order to develop rust-resistant commercial soybean lines, the function of the PR10 protein, which was found expressed either at higher levels or rapidly induced in resistant lines compared to susceptible ones in a time-course proteomics study, was further investigated. A short fragment of PR10 was cloned into a bean pod mottle virus (BPMV) based virus induced gene silencing (VIGS) vector and introduced into soybean to suppress $P R 10$ expression. Rust accumulation increased 5.2 fold in two ASR resistant lines (PI417089A and PI567104B) when the PR10 expression was suppressed by $80 \%$ through VIGS. Small RNA sequence analysis also confirmed the presence of high levels of $P R 10$ specific small RNAs only in soybean leaves treated with VIGS vector containing RP10. Meanwhile, reactive oxygen species (ROS) related microRNAs (miR408, miR398, miR2119) were highly down-regulated in the PR10 silenced leaf samples. PR10 
silenced soybean leaves also produced less ROS after rust inoculation when stained with 3,3'-diaminobenzidine (DAB). These results indicate a direct function of PR10 in conferring ASR resistance possibly through enhancing the production of ROS at the site of rust infection.

Bacillus amyloliquefaciens strains in the management of Cercospora leafspot of lettuce in Trinidad A. Thomas (1), D. SARAVANAKUMAR PHD (2), (1) UWI, Port of Spain, TRINIDAD AND TOBAGO; (2) University of the West Indies, St. Augustine, Trinidad, TRINIDAD AND TOBAGO

Lettuce (Lactuca sativa) is a vegetable crop that is favoured and highly demanded in many cultures around the world and mostly consumed fresh. It is a short crop, meaning, most varieties are harvested within 4-5 weeks after transplant. According to the United States Department of Agriculture (2016), the value of utilized lettuce in 2016 amounted to near \$2 billion in the United States alone, falling second to tomatoes. Within recent times, there has been a growing concern over the use of pesticides and the impact they have on human health and the environment. In Trinidad, the fungal leafspot of lettuce caused by Cercospora lactucae-sativae is a growing concern as the disease severely affects the quality and reduces yield of the crop forcing farmers to resort to the only known remedy of chemical treatment. This research explored the potential of growth and biocontrol efficacy of indigenous Bacillus strains isolated from rhizosphere soils against leafspot disease. Preliminary research has showed the antagonistic potential of Bacillus amyloliquefaciens to inhibit the fungal pathogen by up to $74 \%$ in-vitro and boost the seedling growth (leaf area) by about $300 \%$. The PCR analysis of antibiotic lipopeptides genes viz., Iturin, Bacillomycin, Bacilysin, Fengycin, Surfactin and Zwittermycin in those B. amyloliquefaciens strains were positive. Bacillus species which did not show positive for any of the lipopetides resulted in little or no inhibition, indicating that the antibiotic genes contributed significantly to inhibitory properties of the B. amyloliquefaciens. Further testing of the antagonistic B. amyloliquefaciens strains under protected and field settings is required to understand the biocontrol efficacy against leaf spot disease in lettuce.

\section{Quantitative resistance to bacterial pathogens of rice}

J. E. LEACH (1), A. M. Bossa-Castro (1), A. I. Huerta (1), E. Delorean (1), C. Raghavan (2), C. Tekete (3), A. Dereeper (4), B. W. Tonnessen (1), O. Koita (3), G. M. Mosquera (5), H. Leung (2), V. M. Verdier (4), (1) Colorado State University, Fort Collins, CO, USA; (2) International Rice Research Institute - IRRI, Los Baños, PHILIPPINES; (3) University of Sciences, Techniques and Technologies of Bamako (USTTB), LBMA, Bamako, MALI; (4) IRD, Cirad, Univ Montpellier, IPME, Montpellier, FRANCE; (5) International Center for Tropical Agriculture - CIAT, Palmira, COLOMBIA

Disease resistance is the foundation for managing many plant diseases, because resistant varieties have the strongest impact with minimal environmental effects or cost. Consequently, sources of broad-spectrum resistance (BSR), or resistance that is effective against multiple and/or diverse pathogens is of particular interest. However, achieving BSR depends on having effective resistance sources to introgress into elite germplasm. Multi-parent Advanced Generation Inter-Cross (MAGIC) populations are powerful tools for identifying resistance because they have high levels of recombination and enhanced resolution relative to biparental populations. We screened an indica rice MAGIC population developed from eight elite founders for BSR to diverse strains of the rice bacterial blight and leaf streak pathogens Xanthomonas oryzae pv. oryzae (Xoo) and X. o. pv. oryzicola (Xoc), respectively. In addition, building on our hypothesis that durable disease resistance is attainable by targeting key microbial virulence factors, we screened for resistance to Xoo strains isogenic for the known and common virulence factor TAL 7b. A combination of genome-wide association studies and interval mapping analyses revealed a number of loci that conferred BSR to both Xoo and Xoc, as well as resistance targeted at TAL7b. These BSR QTL are excellent sources for durable, broadly effective resistance in the field.

Identifying molecular components of reduced demethylation inhibitor (DMI) fungicide sensitivity in Blumeria graminis f.sp. tritici E. A. MEYERS (1), R. Whetten (2), C. Cowger (3), (1) Department of Entomology and Plant Pathology, North Carolina State University, Raleigh, NC, USA; (2) USDA-ARS Plant Science Unit, Raleigh, NC, USA; (3) USDA-ARS, Department of Entomology \& Plant Pathology, North Carolina State University., Raleigh, NC, USA

Blumeria graminis f.sp. tritici (Bgt), the causal agent of wheat powdery mildew, occurs annually in states along the eastern seaboard of the United States (US) and infects wheat worldwide. Reduced sensitivity to DMI fungicides has been observed in both European and Australian Bgt populations. The first evaluation of DMI sensitivity in US Bgt populations was conducted with isolates collected from 15 US states. Sensitivity to two DMIs (tebuconazole and prothioconazole) was measured for 380 isolates, with a third DMI (metconazole) measured for 100 isolates. A large range in DMI sensitivity was observed, with a significantly larger reduction in sensitivity observed in eastern US states compared to central Great Plains states. Discovering the molecular drivers of DMI sensitivity in US Bgt populations will assist in addressing fungicide resistance in US wheat production and add to understanding how fungicides influence the global Bgt population. Targeted partial genome sequencing of phenotyped isolates identified genetic components related to the observed differences in DMI sensitivity. Sequencing of the DMI-targeted CYP51 gene revealed a Y136F mutation that significantly correlates with reduced sensitivity to tebuconazole $\left(\mathrm{P}<0.001, \mathrm{R}^{2}=0.11\right)$; however, 17 tebuconazole-insensitive isolates lack the mutation and 67 sensitive isolates have Y136F. Future analyses will evaluate more SNPs for DMI sensitivity association and qPCR analysis of CYP51 for expression and copy number.

Can rainfall be a useful predictor of epidemic risk across temporal and spatial scales?

E. M. DEL PONTE (1), A. H. Sparks (2), N. J. Cunniffe (3), L. V. Madden (4), (1) Universidade Federal de Vicosa, Vicosa, BRAZIL; (2) University of Southern Queensland, Toowoomba, AUSTRALIA; (3) University of Cambridge, Cambridge, UNITED KINGDOM; (4) The Ohio State University, Wooster, OH, USA

Plant disease epidemics are largely driven by within-season weather variables when inoculum is not limiting. Commonly, predictors in risk assessment models are based on the interaction of temperature and wetness-related variables, relationships which are determined experimentally. There is an increasing interest in providing within-season or inter-seasonal risk information at the region or continent scale, which commonly use models developed for a smaller scale. Hence, the scale matching dilemma that challenges epidemiologists and meteorologists: upscale models or downscale weather data? Successful applications may be found in both cases, which should be supported by validation datasets whenever possible, to prove the usefulness of the approach. For some diseases, rainfall is key for inoculum dispersal and, in warmer regions (e.g., tropics) where temperature is less limiting for epidemics, rainfall extends wetness periods. The drawbacks of using rainfall at small scales relate to its discrete nature and high spatial variability. However, for pre- or early-season predictions at large spatial scales sources of reasonably accurate rainfall summaries are available and may prove useful. The availability of disease datasets at various scales allows the development and evaluation of new models to be applied at the correct scale. We will showcase examples and discuss the usefulness of rainfall as key variable to predict soybean rust and wheat scab from field to region.

Development of a quantitative PCR-based method for the detection and monitoring azoxystrobin resistance in Pyricularia oryzae populations A. KUNOVA, C. Pizzatti, M. Pasquali, P. Cortesi, DeFENS, Università degli Studi di Milano, Milano, ITALY 
Rice blast, caused by Pyricularia oryzae, is one of the most important rice diseases worldwide, causing economically important rice yield losses and representing a threat to global food security. Rice blast management relies on fungicides, especially in Europe, as susceptible rice varieties are intensively grown for their quality value. Azoxystrobin (a Quinone outside inhibitor fungicide, QoI) represents often the first - and sometimes the only choice of chemical control of rice blast. However, it is a fungicide with a high risk of resistance development in pathogen populations. Indeed, $P$. oryzae azoxystrobin-resistant strains from rice have been identified in Japan, which poses concerns about the spread of QoI resistance also in other rice-growing areas. Despite this, no reliable and sensitive detection method of QoI resistance in P. oryzae populations exists at the moment. We developed two qPCRbased methods for the detection of QoI-resistant strains of P. oryzae. One is based on selective amplification of sensitive (S) or resistant (R) allele using S- and R-specific primers, while the other exploits high resolution melting analysis of the PCR product. Here, we evaluate and compare the sensitivity and specificity of the two methods using DNA mixtures of S and R strains at different ratios. This method is to be used where QoI application is the predominant means of rice blast management, to monitor the emergence of $P$. oryzae QoI-resistant strains.

\section{The International Plant Protection Convention and its common ground with plant pathologists to contribute to plant health and wildlife, in the world \\ J. Trujillo (1), C. GARCÍA-AVILA (2), (1) NPPO of Mexico (SAGARPA-SENASICA), Coyoacán, MEXICO; (2) SENASICA-DGSV, Estado de México, MEXICO}

The International Plant Protection Convention (IPPC) is a 65 years old international treaty among 183 contracting parties, whose mandate is the global adoption of phytosanitary principles and measures, aimed to protect the plant resources of the world, both agricultural and natural. The IPPC it is recognized by the World Trade Organization as one of the three standard setting organizations. The development of international standards of phytosanitary measures (ISPMs) includes, thus far, 94 standards; including 41 ISPMs, 22 Diagnostic Protocols and 31 Phytosanitary Treatments. In order to achieve effectiveness, it balances legal and scientific criteria. So that, regular interaction with scientists, individually and organizations, becomes an invaluable mechanism, that needs to be regular and robust in the long haul. Examples of this interaction include, in the past, Phytophthora infestans, Erwinia amylovora, Clavibacter michiganensis and Mycosphaerella fijiensis. Presently, Xylella fastidiosa, citrus greening and FOCR4T, in some countries. In the future, it is forseen the need to interact around themes like virus diseases, Xylella fastidiosa subsp. multiplex, UG99 rust and Phytophthora ramorum. The governing body of the IPPC, the Comission for Phytosanitary Measures has launched, supported by FAO, an initiative for the year 2020 to be recognized by the UN, as the is International Year of Plant Health.

\section{Caliciopsis canker: An emerging disease in pine stands}

N. LUCHI (1), C. Aglietti (2), D. Migliorini (3), A. Pepori (1), P. Capretti (2,4), I. A. Munck (5), K. D. Broders (6), A. Santini (1), (1) Institute for Sustainable Plant Protection - CNR, Sesto fiorentino, ITALY; (2) University of Firenze, Firenze, ITALY; (3) Forestry and Agricultural Biotechnology Institute, University of Pretoria, Pretoria, SOUTH AFRICA; (4) University of Firenze - DISPAA, Firenze, ITALY; (5) USDA Forest Service, Durham, NH, USA; (6) Colorado State University, Fort Collins, CO, USA

Caliciopsis canker, caused by Caliciopsis pinea, have been increasingly observed in plantations of Pinus radiata in Italy, and occasionally on $P$. pinaster. In Europe the disease has been described since 1896 on $P$. mugo, and has also been reported on $P$. radiata and native species, such as $P$. pinea, $P$. pinaster and $P$. halepensis. In North America the fungus was described since 1920, and recently has been reported as an emerging problem in $P$. strobus plantations in Eastern North America. In 2010 in Spain C. pinea was found on the same stem of a P. radiata tree that was also infected by Fusarium circinatum, an invasive alien pine pathogen introduced in Europe in the early 2000s. Aim of this work was to increase knowledge on this emerging disease, by analyzing the phylogeny of European (E) and North American (NA) pathogen populations, challenging the pathogenicity on different native and non-native hosts, and assessing whether a possible association with $F$. circinatum exists. European isolates showed no genetic variability and clustered with a single NA provenance. In contrast NA strains are much more variable. C. pinea was able to cause significant lesions on seedlings of different pine species. Contrasting results were obtained as regards of association with $F$. circinatum: different $C$. pinea isolates react differently to its presence. The uniformity of the European population suggests an introduction event, probably from North America. It is possible that Caliciopsis canker could become a dangerous pathogen of pine stands in Europe, especially if they suffer for environmental stresses due to climate change. Finally, $F$. circinatum seems to induce the production of ligninases by some $C$. pinea strains, suggesting a fungus-fungus interaction.

\section{Critical aspects of biologically relevant seed health assays}

C. M. Vera Cruz (1,2), M. H. R. Nguyen (1), J. M. Lang (3), B. Cottyn (4), V. M. VERDIER (5), D. Mishra (6), Y. Raj (6), J. E. Leach (3), (1) International Rice Research Institute, Los Baños, Laguna, PHILIPPINES; (2) Los Baños, Laguna, PHILIPPINES; (3) Colorado State University, Fort Collins, CO, USA; (4) ILVO, Merelbeke, BELGIUM; (5) IRD, Cirad, Univ Montpellier, IPME, Montpellier, FRANCE; (6) Bayer CropScience, Hyderabad Andhra Pradesh, INDIA

There is no question on the importance of preventing the spread of plant pathogens across the globe. This is facilitated by the creation and implementation of phytosanitary regulations, and the continuous improvement of seed health testing protocols. Our experience in developing an assay for detection of Xanthomonas oryzae pvs. oryzae and oryzicola rice seed contamination has shown us that this is not an easy task. Critical aspects of seed health assays are not only limited to the technical specifications of the detection method, such as sensitivity, selectivity, ruggedness, and reliability. In diagnostic PCR, the inclusion of test controls (i.e. internal amplification control, process controls, no template controls, positive controls, and non-target controls) are necessary to validate any result. Also, the simplicity of an assay is important for it to be useful. Is the required equipment commonly available or too specialized? Are the extraction steps simple and easy to carry out? In the final stages before protocol dissemination to users, the protocol should be audited by a higher body, such as the International Seed Testing Association (ISTA), and the accreditation of a protocol for universal use would depend on stringent evaluation by ISTA. Developing protocols for seed health testing should be done with the prime intention to create a method for easy adoption by quarantine laboratories across the globe.

Survey and Identification of Meloidogyne Species Associated with Potato in North Sumatra, Indonesia

L. LISNAWITA (1), F. Kurniawati (2), A. R. Tantawi (3), H. Yusuf (1), E. A. Nainggolan (1), (1) Universitas Sumatera Utara, Medan, INDONESIA; (2) Bogor Agricultural University, Bogor, INDONESIA; (3) University of Medan Area, Medan Estate, INDONESIA

Root knot nematode (RKN/Meloidogyne spp.) is one of the main constraint of potato production in Indonesia, especially in Karo District as potato production centre in North Sumatra. A survey in 9 localities of potato-growing area of Karo District was conducted to identify species of root-knot nematode associated with potato. Morphological characters based perrineal pattern, and DNA sequence analysis were used to identify the nematodes. Soil and root samples were collected from farmer's fields. Single egg mass was identified by morphological studies and then confirmed by PCR amplification of DNA using species-specific SCAR (sequence characterized amplified region) primers, sequencing, and NCBI-BLASTn analysis of the 
target product. The results showed 3 species found in Karo District. They are M. arenaria, M. incognita, and M. javanica. The species were either found singly or in mixed populations. This is the first report of Meloidogyne species associated with potato in North Sumatra, Indonesia.

Genotyping by sequencing to identify diagnostic regions in Fusarium oxysporum f.sp. cubense Tropical Race 4 and applications in disease epidemiology

M. SALACINAS (1), N. Ordonez (1), O. Mendes (2), C. D. Schoen (2), M. Seidl (1), H. Meijer (2), G. H. J. Kema (1,2), (1) Wageningen University and Research, Wageningen, NETHERLANDS; (2) Wageningen Plant Research, Wageningen, NETHERLANDS

The recent damage by Fusarium oxysporum f.sp. cubense Tropical Race 4 (Foc-TR4) is alarming as it destroys banana intended for domestic consumption and international trade. Containment of the pathogen is of prime importance thus strict quarantine measures have to be implemented requiring accurate detection. Genotyping by sequencing based on Diversity Array Sequencing Technology (DArT) and subsequent in silico analyses were utilized to identify TR4 specific genomic sequences. A single copy and non-coding genomic region was identified as TR4 unique region. From this, primers for DNA-based diagnostics were developed for Loop Mediated Isothermal Amplification (LAMP) and TaqMan probe-based diagnostics. The low-throughput LAMP assay successfully detect TR4 in infested banana tissues within minutes. TaqMan assays were used to detect soil and water infestations, revealing that irrigation water are potential source of inoculum for further spread of TR4 in disease free areas. Mapping TR4 spatial distribution in banana and non-banana soils revealed TR4 inoculum until $100 \mathrm{~cm}$ below the surface. Furthermore, using the developed diagnostics we showed that traffic and labor in contaminated farms contributes to the dissemination of TR4 by detection of the pathogen in the footwear used in the farm, which might contribute to regional, intra-and inter-continental dissemination of TR4. Thus, the developed diagnostics are indispensable in addressing epidemiological questions related to the spread of this pathogen and provided information for banana farmers, companies, and producers to initiate improved surveillance and management strategies.

Rare Sugar: A Novel Signal Molecule for Growth Inhibition and Defense Induction in Plants K. AKIMITSU, Kagawa University, Miki, Kagawa, JAPAN

Rare sugars including D-allose, an epimeric monosaccharide at C3 of D-glucose, are defined as monosaccharides with low abundance in nature. Among several rare sugars, D-allose inhibits the gibberellin-dependent responses such as elongation of rice leaves, and induces resistance to Xanthomonas oryzae pv. oryzae in susceptible rice leaves with defense responses: accumulation of reactive oxygen species, lesion mimic formation, and PR-protein gene expression. D-Allose suppresses expressions of gibberellin-responsive genes at downstream of SLR1 protein through hexokinase (HXK)-dependent pathway. D-Allose failed to trigger growth retardation in Arabidopsis glucose-insensitive2 (gin2) mutant, which is a loss-of-function mutant of AtHXK1. D-Allose effects were recovered in transgenic gin2 over-expressing wild-type AtHXK1 but not in gin2 over-expressing the catalytic mutant AtHXK1S177A, indicating that the D-allose phosphorylation by HXK to D-allose 6-phosphate (A6P) is essential for D-allose-induced growth inhibition. D-Allose-mediated defense responses were suppressed by the presence of a HXK inhibitors. Transgenic rice plants expressing Escherichia coli AlsK encoding D-allose kinase to increase A6P synthesis were more sensitive to D-allose, but E. coli AlsI encoding A6P isomerase expression to decrease A6P reduced its sensitivity. These findings indicated that the phosphorylation of D-allose at C6 by HXK is also essential for the induction of defense reactions following rice resistance to the pathogen. This molecule may be a candidate for new type of growth regulator with defense induction for plant such as turf grass.

Tracking pests and insect-vectored diseases of broad-acre crops in tropical Asia: Challenges \& opportunities K. Wyckhuys (1), B. DHARANI DHAR (2), (1) Institute of Plant Protection - China Academy of Agricultural Sciences CAAS, Beijing, CHINA; (2) International Center for Tropical Agriculture - Asia, Hanoi, VIETNAM

Cassava (Manihot esculenta Crantz) is one of the world's lead food, feed, fiber and bio-energy crops. Cultivated by $>8$ million farmers across tropical Asia, the crop plays a central role in the rural economy of countries such as Vietnam, Thailand, Indonesia or Cambodia, and underpins a multi-billion dollar regional starch industry. While Asia's cassava crops had historically remained free from phytosanitary threats, they have recently been devastated by invasive pests and insect-vectored diseases. Diseases such as the whitefly-vectored cassava mosaic virus (CMV) or the phytoplasma-associated cassava witches broom (CWB) have rapidly spread through tropical Asia, with their propagation facilitated by the un-checked, cross-boundary movement of planting material. Local plant health authorities face major hurdles to monitor these biotic threats, adopt appropriate quarantine measures to halt their spread, or mitigate their on-farm impact. In this presentation, we provide a first-hand update on the geographical expansion of cassava diseases, describe status of ongoing (multi-country) mitigation efforts, and outline priorities for applied research. We illuminate lucrative opportunities to deploy a) simulation modeling to guide pest surveillance and biosecurity action, b) remote sensing and drone-based approaches to track disease progression, and c) in-depth studies of vector ecology, e.g., to anticipate disease spread based upon presence and relative abundance of vector populations.

Exploring genebank for identification of biotic-abiotic combined tolerance in wild Phaseolus G. MOSQUERA CIFUENTES (1), C. Cotes (1), V. Arredondo (1), S. Beebe (1), S. Barrera (2), (1) CIAT, Palmira, COLOMBIA; (2) International Center for Tropical Agriculture (CIAT), Cali, COLOMBIA

Climate change is an important factor that is impacting production and distribution of beans, affecting directly plant development and indirectly by influencing changes in disease patterns. By 2020, an increase in precipitation would be expected in many African countries, and under these conditions root rot diseases will be favored. In order to address these limitations, it is necessary to find new genetic variants that could be used by breeding programs to develop better varieties resilient to climate change. Wild bean relatives are a useful resource that must be explored more extensively, since they harbor genes important for bean adaptation to diverse environments, and can be used to improve cultivated beans with superior performance against abiotic and biotic stress. More than 150 accessions from including Phaseolus vulgaris, Phaseolus coccineus, Phaseolus dumosus, Phaseolus costaricensis and Phaseolus albescens were phenotyped under greenhouse conditions for waterlogging tolerance and Pythium myriotylum resistance. Some accessions from $P$. vulgaris, $P$. coccineus, and $P$. dumosus showed to be tolerant to waterlogging and resistant to $P$. myriotylum at seedling stage. In total 6 accessions were crossed with SMR138 and BFS142, two bean breeding lines with superior quality as acceptable grain type, high yield, high iron content, low fertility tolerance. Populations are being advanced in order to transfer the resistant into elite lines to obtain bean lines tolerant to waterlogging and $P$. myriotylum resistance. Our research demonstrates the relevance of exploring gene banks as source of agronomic traits for breeding programs, being crop wild relatives an interesting resource waiting to be characterized and used to develop better varieties able to endure environmental constraints associated to climate change.

Molecular characterization of Fusarium mangiferae associated with malformation of mango in Pakistan

S. IRAM, Fatima Jinnah Women University, Rawalpindi, Rawalpindi, PAKISTAN 
Mango malformation is considered as the most devastating disease of mango. The present study has assessed the prevalence and severity of mango malformation in Punjab province (Pakistan) as well as molecular characterization of fungal pathogens associated with mango Malformation. A survey was conducted in 3 districts of Punjab that are Multan, Khanewal and Muzafargarh. Thirty eight orchards were visited in three districts of the Punjab province to confirm the status of mango malformation. Hundred percent prevalence was shown in Muzafargarh and Multan. The causative pathogens of malformation were isolated and morphologically identified by determining cultural characteristics. One specie from genus Fusarium, "Fusarium mangiferae", was identified and its identification was confirmed by molecular sequencing analysis. A pair of ITS primer was used to amplify the obtained DNA. The amplified PCR products were resolved using $2 \%$ agarose gel, stained with ethidium bromide. Isolates' band size was in range 580 600 base pair. The amplified products from ITS-5.8S-rDNA were sequenced and then compared to ITS sequences of isolated species from NCBI which showed 97 to $100 \%$ similarity with Fusarium magnifierae. Sequences obtained in this study were aligned and a neighbor joining tree was rooted to determine phylogenetic relation among the isolate of detected specie and their similar species. Results obtained through this work would be supportive in establishment of association of isolates with their region and also helpful to device management strategies to decrease the mango diseases and disorders in orchards caused by Mango Malformation.

Insights into fruit defense mechanisms against the main post-harvest pathogens of apples and oranges R. TORRES, L. Vilanova, J. Giné-Bordonaba, N. Teixidó, N. Vall-Llaura, C. Larrigaudière, J. Usall, IRTA, XaRTA-Postharvest, Edifici Fruitcentre, Lleida, Catalonia, SPAIN

Despite the current use of chemical fungicides, Penicillium digitatum and Penicillium expansum, the most devastating pathogens of citrus and pome fruits, respectively, are still responsible of important economic losses during postharvest handling worldwide. Current trends are focused on finding new rational and environmental friendly control alternatives. In this sense, a better understanding of the fruit-pathogen interaction may be consider as a novel perspective for the control of postharvest diseases. The main goal of these studies was to obtain a better understanding of the fruit's defence response against both pathogens. For such purposes, both the lignification process and the role of ethylene production on compatible and non-host interactions was studied using pathological, biochemical and molecular approaches. Concerning lignification, the main results indicate that orange response to wounding is slower than the response to the pathogen attack. In apples, the resulting data from transcriptomic analysis provide further evidence that apples inoculated with $P$. expansum exhibit significant upregulation of defence-related genes and genes involved in detoxification of reactive oxygen species. The results related to ethylene biosynthesis on apples showed that $P$. expansum and $P$. digitatum could differentially alter the fruit ethylene production, probably by the manipulating the endogenous fruit ethylene biosynthesis, leading to the circumvention or suppression of effective defences and hence facilitating its colonization.

Soil fauna effects on degradation of Fusarium graminearum mycotoxins in contaminated plant residues at different temperature regimes F. MEYER-WOLFARTH (1), S. Schrader (2), E. Oldenburg (3), K. Munoz (4), T. Meiners (5), (1) Johann Heinrich von Thuenen-Instiute of Biodiversity, Braunschweig, GERMANY; (2) Johann Heinrich von Thuenen-Institute of Biodiversity, Braunschweig, GERMANY; (3) Julius-KuehnInstitute of Plant Protection in Field Crops and Grassland, Braunschweig, GERMANY; (4) Univerity of Koblenz-Landau, Landau, GERMANY; (5) Julius-Kuehn-Institute of Ecol. Chemistry, Plant Analysis \& Stored Product Protection, Berlin, GERMANY

Infection of cultivated plants with toxigenic fungal pathogens may lead to increased mycotoxin levels in crop products and residues. By leaching from host tissue of infected plants, mycotoxins can enter soil and water as potential environmental contaminants. In agroecosystems, fungivorous soil fauna can be antagonistic to pathogen infection and mycotoxin contamination and act as biological and chemical regulators. Therefore, a microcosm-study with earthworms (Aporrectodea caliginosa) and collembolans (Proisotoma minuta) was conducted in a climate chamber to assess their biocontrol-impact on the fungal plant pathogen Fusarium graminearum and the content of its mycotoxins deoxynivalenol (DON) and zearalenone (ZEN) in maize stubbles, soil, and leaching water. Soil fauna was exposed to artificially infected maize stubbles highly contaminated with DON $\left(10,462 \mu \mathrm{g} \mathrm{kg}^{-1}\right)$ and ZEN $(2,780$ $\mu \mathrm{g} \mathrm{kg}^{-1}$ ) at $17^{\circ} \mathrm{C}$ or $25^{\circ} \mathrm{C}$. After 6 weeks, maize, soil, and leaching water samples were analysed for mycotoxins via liquid chromatography coupled to high resolution mass spectrometry. First results indicate significant faunal effects of both introduced species on DON reduction in residual maize. At $25^{\circ} \mathrm{C}$, DON was reduced most in mixed faunal treatment compared to the non-faunal control. A faunal effect on ZEN reduction was not observed. The introduced soil fauna contributes to the natural self-regulation in the soil system. However, their biocontrol potential is influenced by temperature.

Ending the game of cat-and-mouse between tobamoviruses and their resistance genes

S. TSUDA (1), K. Kubota (2), (1) Central Region Agricultural Research Center, NARO, Tsukuba, Ibaraki, JAPAN; (2) Central Region Agricultural Research Center (NARO), Tsukuba, Ibaraki, JAPAN

Resistance genes are powerful tools to control plant viral diseases, against which no agricultural chemicals are available. However, viruses can obtain the abilities to overcome those resistance gene(s) through changes in their genomes, such as recombination or nucleotide substitutions. The genome of tobamoviruses can encode avirulence factors that elicit resistance controlled by a cognate dominant host gene, viral coat protein for four alleles consisting of $L^{1}$ to $L^{4}$ in pepper, and viral movement protein for two alleles of $T m-2$ and $T m-2^{2}$ in tomato, for instance. In green pepper cultivation fields in Japan, the $L^{3}$ resistance gene can successfully prevent tobamoviral diseases in most cases. However, a virulent isolate of tobamoviruses has developed the ability to overcome the $L^{3}$ resistance gene. Similarly, another variant has been discovered that is able to overcome the $L^{4}$ resistance gene in the past decade. In tomato cultivation, emergence of several resistance-breaking tobamovirus variants has sometimes been found in Japan, which are capable of infecting tomato cultivars carrying resistant genes; $T m-2$ and/or $T m-2^{2}$. Experts should make effort to achieve successful management of these resistance genes for sustainable crop production, which must be supported by integrated pest management strategies and scientifically sound evidences, although technological strategies applicable to control viral diseases in field are currently limited. 